
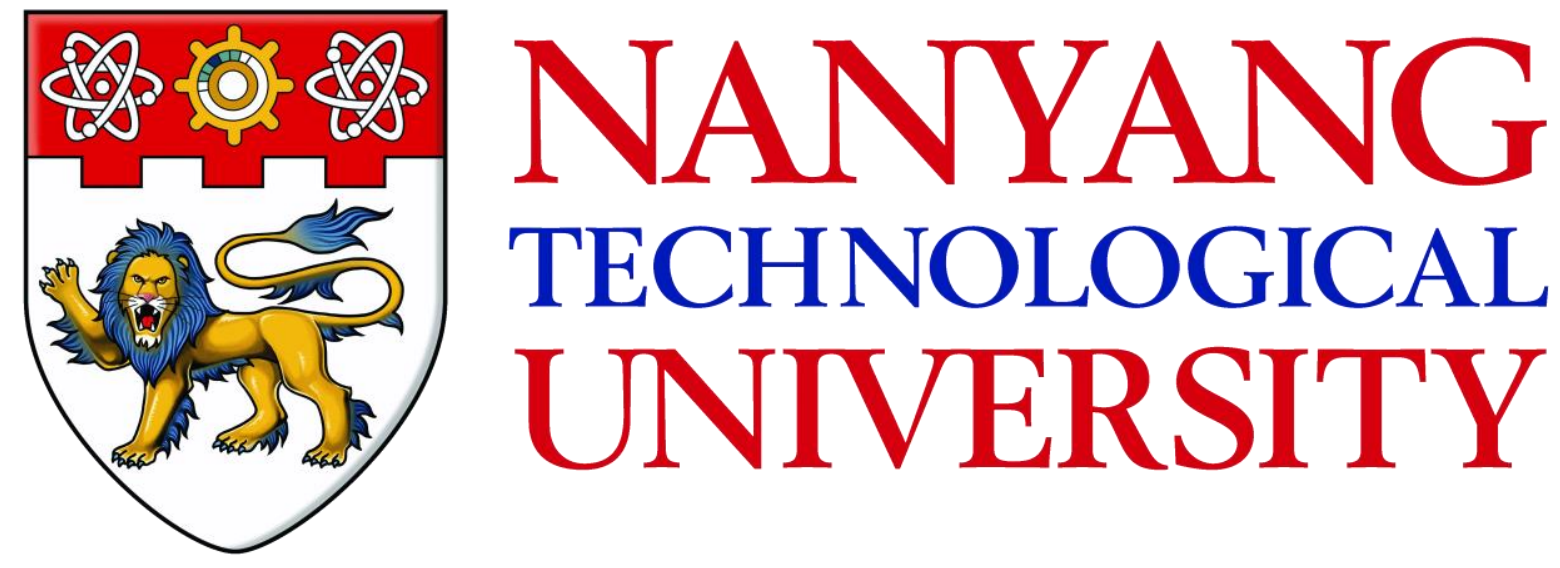

ROLE OF MITOPHAGY IN

MUSCLE GROWTH AND METABOLISM

NESIBE PEKER

SCHOOL OF BIOLOGICAL SCIENCES

2017 


\section{ROLE OF MITOPHAGY IN \\ MUSCLE GROWTH AND METABOLISM}

NESIBE PEKER

SCHOOL OF BIOLOGICAL SCIENCES

A thesis submitted to the Nanyang Technological University in partial fulfilment of the requirement for the degree of Doctor of

Philosophy

\section{7}




\section{ACKNOWLEDGEMENTS}

This thesis, describing a novel role of Parkin function in skeletal muscle, has been completed by contribution of many people. First of all, I would like to express the deepest appreciation towards my supervisor Doctor Craig McFarlane, for his support, intellectual guidance and critical advises. His guidance and constructive critiques made me a competent scientist and better writer. At the end of four year, I feel these words may not be sufficient to express my gratitude towards him, but being an excellent scientist and mentor like him in the future.

My appreciation also extends to Professor Ravi Kambadur, who mentored me for almost 2,5 years with his brilliant ideas and suggestions that shaped this thesis.

I would like to also thank Professor Ajai Vyas and Professor Koh Cheng Gee for their administrative support and timely help.

I have appreciated histological analysis and confocal imaging performed by the help of Vinay Guru Donipadi and NUS Confocal Microscopy Unit.

I gratefully acknowledge the funding sources that made my Ph.D. work possible. I was funded by the Turkish Education Foundation and Singapore International Graduate Student Award (TEV-SINGA). My work is also supported by Nanyang Technological University and A*STAR.

Also, I am indebted to my parents and brother who endured and answered all my insisting questions, during my childhood, which encouraged me to ask the question "Why?" more. Also, I would like to thank to my twin sister, my other half, my best friend, for her love and encouragement.

Finally, to eternal knowledge and science,

From a priori to a posteriori... 


\section{TABLE OF CONTENTS}

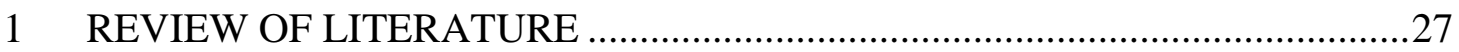

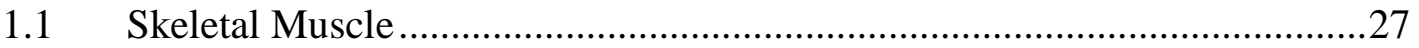

1.1.1 Skeletal Muscle and Muscle Cell Structure ...........................................22

1.1.2 Muscle as a Contractile Tissue ...........................................................29

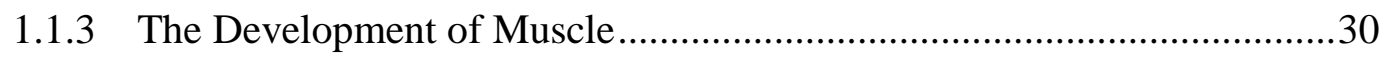

1.1.3.1 Embryonic and Perinatal Myogenesis .............................................30

1.1.3.2 Postnatal Myogenesis and Muscle Regeneration..............................32

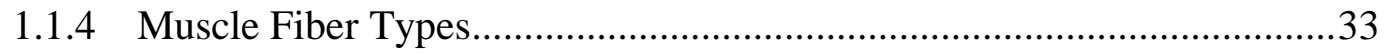

1.2 Muscle Wasting in Skeletal Muscle ...........................................................34

1.2.1 Different Types of Muscle Wasting .........................................................34

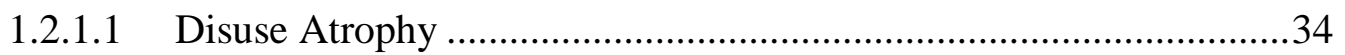

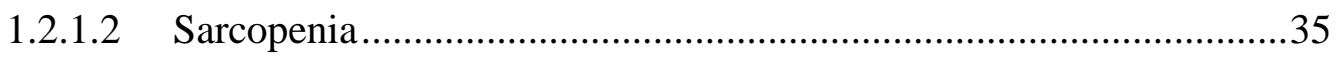

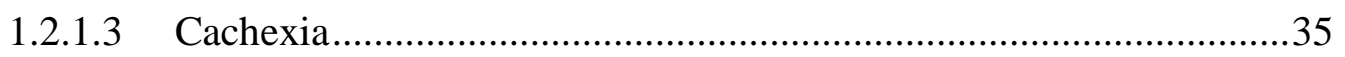

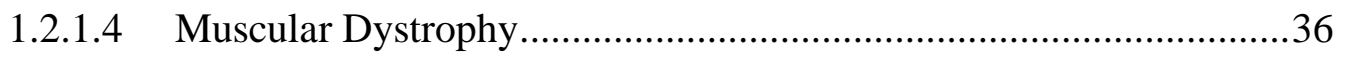

1.3 Proteostasis in Skeletal Muscle Mass ..........................................................37

1.3.1 Protein Synthesis in Muscle Mass Maintenance.......................................37

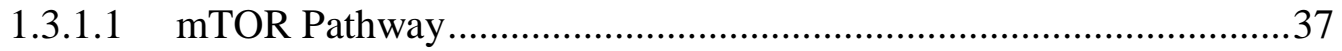

1.3.1.2 $\beta$-catenin/c-Myc Pathway …..........................................................39

1.3.2 Protein Breakdown in Muscle Mass Maintenance...................................39

1.3.2.1 Lysosomal Proteases and Calcium Dependent Calpains ..................40

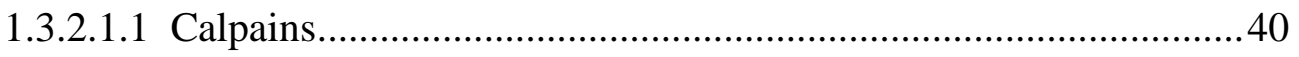

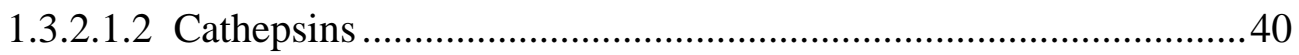

1.3.2.2 Ubiquitin-Proteasome System .....................................................41

1.3.2.2.1 Atrogin-1/MAFbx and MuRF1 .....................................................43

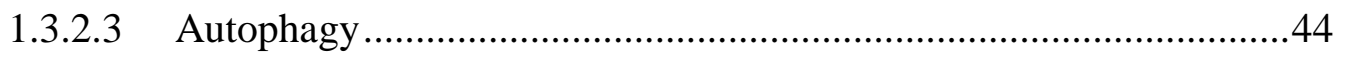

1.3.3 Systemic Mediators of Muscle Wasting ..............................................45

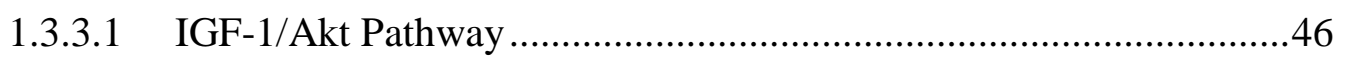

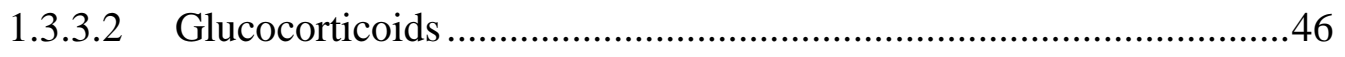

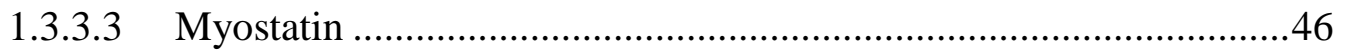

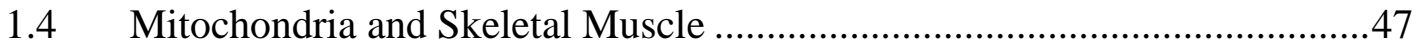

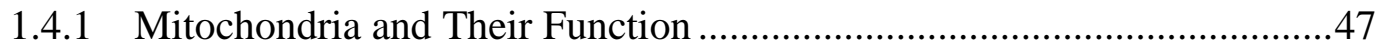


1.4.1.1 Electron Transport Chain and Oxidative Phosphorylation (OXPHOS) 48

1.4.1.2 Detoxification of Reactive Oxygen Species (ROS) .........................49

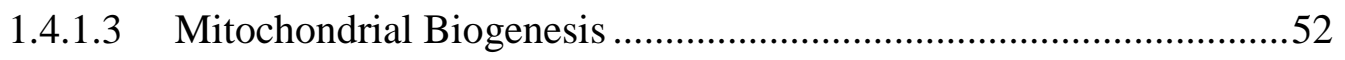

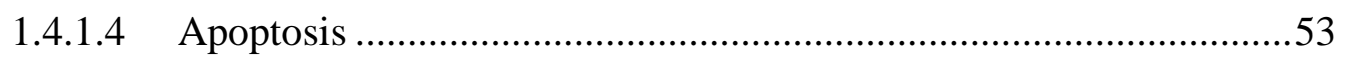

1.4.2 Importance of Mitochondria for Skeletal Muscle ....................................53

1.4.3 Mitochondrial Quality Control ….........................................................54

1.4.3.1 Mitochondrial Proteasome System ................................................55

1.4.3.2 Mitochondria Derived Vesicles (MDV) .........................................55

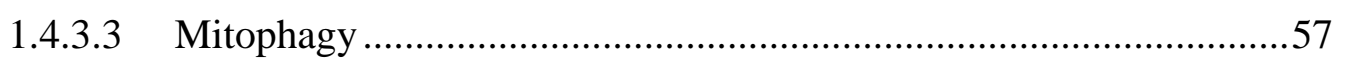

1.4.3.3.1 PINK1/PARKIN-Mediated Mitophagy .....................................59

1.5 Parkinson's Disease and Mitochondrial Dysfunction.................................63

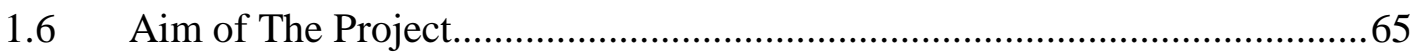

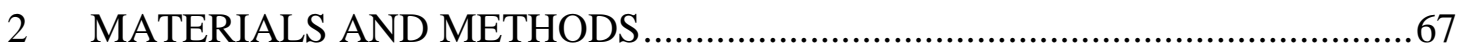

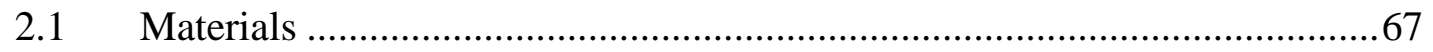

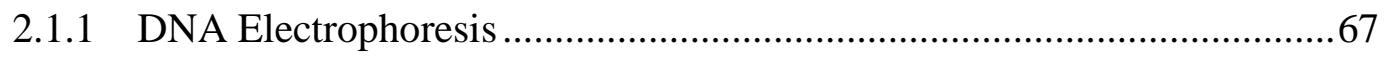

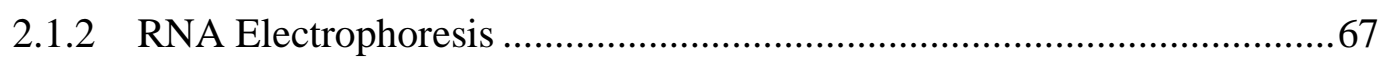

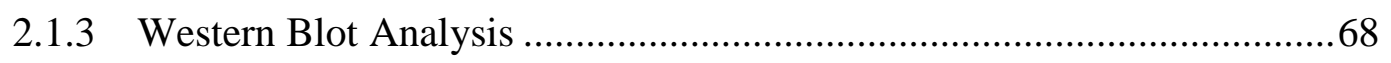

2.1.4 MitoTracker Red CMXRos Confocal Analysis ......................................70

2.1.5 Hematoxylin and Eosin (H\&E) Staining ............................................. 71

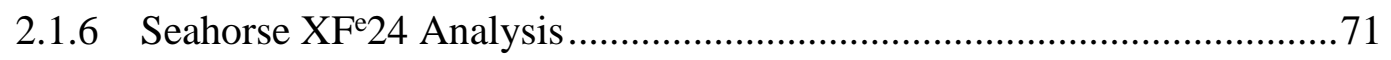

2.1.7 SMARTPool Small interfering RNA (siRNA) Resuspension ...................72

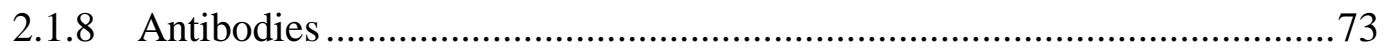

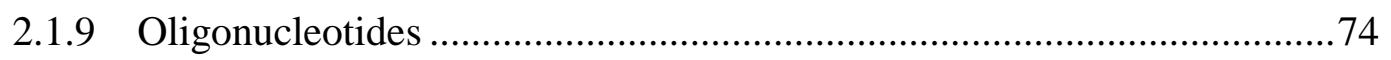

2.1.10 Chemical, Reagent and Kit Inventory List .......................................76

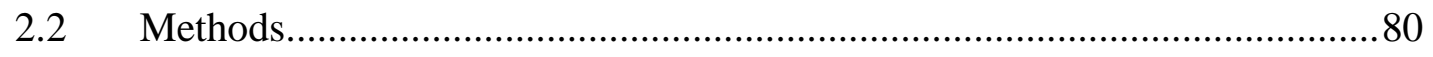

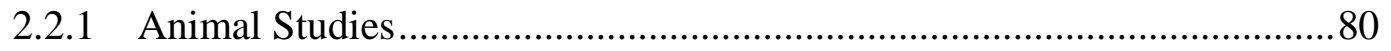

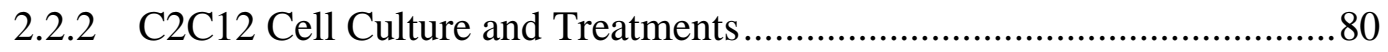

2.2.3 Transient Transfection of SMARTpool siRNA into C2C12 Myoblasts and

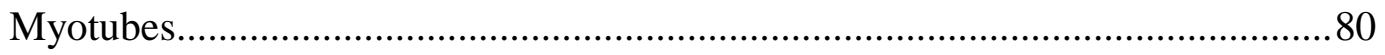

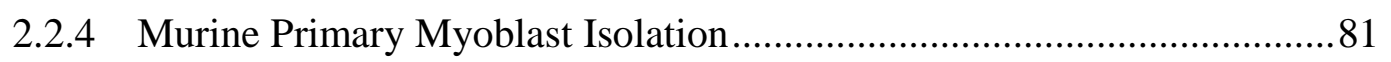

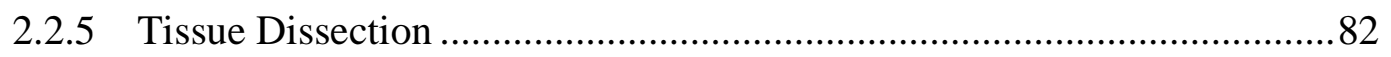

2.2.6 Protein Extraction and Western Blot Analysis .....................................82 
2.2.7 RNA Extraction and Real Time Quantitative PCR (qPCR) .84

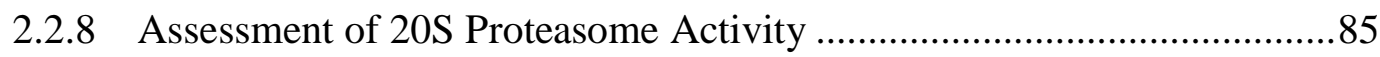

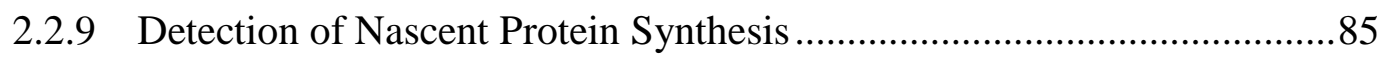

2.2.10 Assessment of Mitochondrial Respiratory Capacity ............................87

2.2.11 Measurement of Exogenous Fatty Acid Oxidation..............................8

2.2.12 MitoTracker Red CMXRos Staining and Confocal Microscopy _.........88

2.2.13 FACS Analysis of MitoTracker Green FM Stained Myoblasts............89

2.2.14 Assessment of Cellular ROS Production ...........................................89

2.2.15 Genomic DNA Isolation and mtDNA Copy Number Analysis ............89

2.2.16 Hematoxylin and Eosin (H\&E) Staining and Myotube Area Analysis 90

2.2.17 H\&E Staining of Tibialis Anterior (TA) Muscle and Cross-Sectional

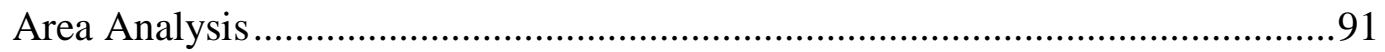

2.2.18 Total RNA Isolation for Microarray and In Silico Analysis.................92

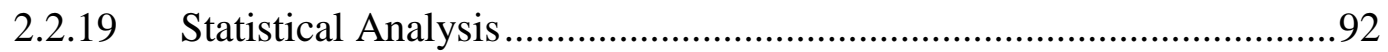

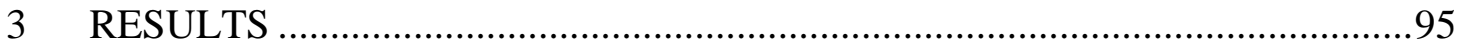

3.1 Treatment with CCCP promotes PINK1/PARKIN-mediated mitophagy in C2C12 myotubes.

3.2 CCCP treatment leads to reduced mitochondrial mass and abnormal mitochondrial morphology in vitro

3.3 CCCP treatment leads to impaired mitochondrial respiratory capacity and reduced utilization of exogenous fatty acids in vitro 103

3.4 CCCP treatment activates the energy sensor AMPK $\alpha$ in vitro.................... 103

3.5 C2C12 myotubes develop ER stress response upon CCCP treatment........ 104

3.6 Treatment of myotube cultures with CCCP leads to myotubular atrophy.. 106

3.7 CCCP treatment activates Akt-FoxO pathway and expression of atrogenes 106

3.8 Knock down of Parkin results in accumulation of dysfunctional mitochondria in myoblast cultures. 109

3.9 Parkin knock down results in impaired mitochondrial turnover ................ 110

3.10 Knock down of Parkin does not alter autophagy in vitro .......................... 114

3.11 Loss of Parkin leads to reduced mitochondrial respiratory capacity, increased cellular ROS levels and activation of AMPK 114

3.12 Parkin knock down results in myotubular atrophy in vitro 118 
3.13 Knock down of Parkin increases MURF1 protein levels and 20S proteasome activity 120

3.14 Protein synthesis remains unaltered upon knock down of Parkin

3.15 Mitochondrial number is preserved and mitochondrial OXPHOS gene

expression is reduced in Parkin KO primary myotube cultures

3.16 Parkin KO primary myotubes have impaired mitochondrial turnover.

3.17 Reduced expression and protein levels of critical OXPHOS genes is observed in Parkin knock out Gas muscle.

3.18 Knock out of Parkin leads to impaired mitochondrial turnover but not autophagy in muscle

3.19 Knock out of Parkin results in reduced mitochondrial function in primary myotube cultures.

3.20 Absence of Parkin results in reduced body weight, decreased skeletal muscle weights and myofiber atrophy in mice

3.21 The myofiber atrophy phenotype observed in Parkin KO muscle tissue may be, at least in part, due to increased MURF1 levels.

3.22 Genes involved in metabolic pathways are downregulated in Parkin $\mathrm{KO}$

skeletal muscle

3.23 Expression of several ubiquitin E3 ligases and E2 ligases are increased in

Parkin KO skeletal muscle

4 DISCUSSION

4.1 CCCP induces PINK1/PARKIN mediated mitophagy in C2C12 myotubes 147

4.2 Loss of Parkin also results in mitochondrial dysfunctional and altered mitochondrial fusion/fission in skeletal muscle

4.3 CCCP treatment results in atrophy phenotype in vitro ...............................152

4.4 Loss of Parkin Leads to skeletal muscle atrophy in vitro and in vivo........153

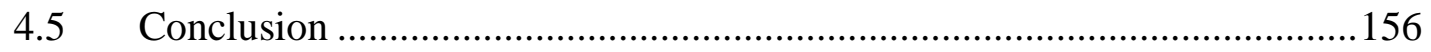

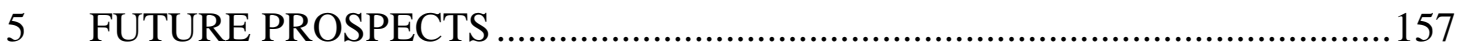

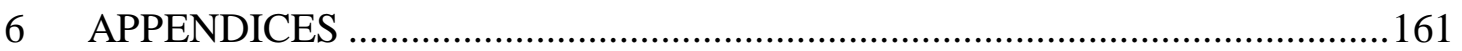

Appendix 6.1: Copyright clearance obtained for Figure 1.3 .................................162

Appendix 6.2: Copyright clearance obtained for Figure 1.4 .................................. 163

Appendix 6.3: Copyright clearance obtained for Figure 1.5 …............................ 164 
Appendix 6.4: Copyright clearance obtained for Figure 1.6 165

Appendix 6.5: Copyright clearance obtained for Figure 1.7 166

Appendix 6.6: Copyright clearance obtained for Figure 2.1 167 


\section{LIST OF FIGURES}

Figure 1.1: Schematic representation of skeletal muscle structure …..........................28

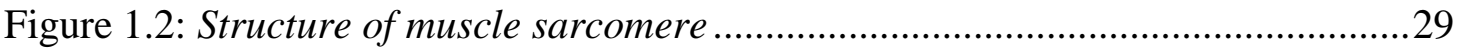

Figure 1.3: Skeletal muscle development ….................................................................

Figure 1.4: mTOR pathway in skeletal muscle protein synthesis.................................39

Figure 1.5: Ubiquitin proteasome system machinery .............................................42

Figure 1.6: Structure of mitochondrion ....................................................................4

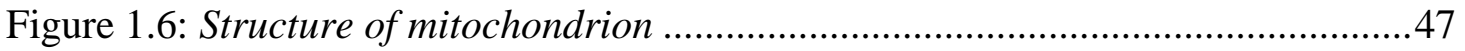

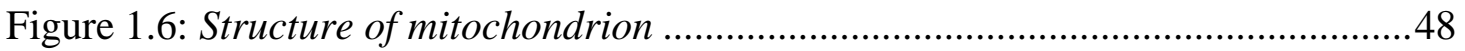

Figure 1.7: Electron transport chain (ETC) in mitochondrial inner membrane ..........51

Figure 1.8: Mitochondrial quality control ................................................................56

Figure 1.9: Graphical model of mitochondrial quality control ....................................58

Figure 1.10: Mitochondrial turnover and mitophagy ...............................................62

Figure 2-1: Representation of mitochondrial respiration parameters..........................87

Figure 3.1: CCCP induces PINK1/PARKIN-mediated mitophagy in C2C12 myotubes

Figure 3.2: CCCP treatment leads to reduced mitochondrial mass and fragmented mitochondrial network.

Figure 3.3: CCCP treatment results in mitochondrial dysfunction in C2C12 myotubes.

Figure 3.4: CCCP-treatment results in endoplasmic reticulum (ER) stress in C2C12

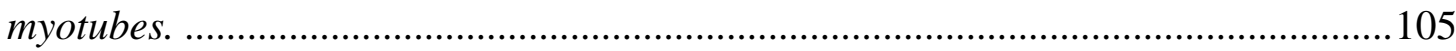

Figure 3.5: CCCP treatment leads to myotube atrophy in vitro...............................107

Figure 3-6: CCCP-treatment activates the transcription factor FoxO3 and enhances

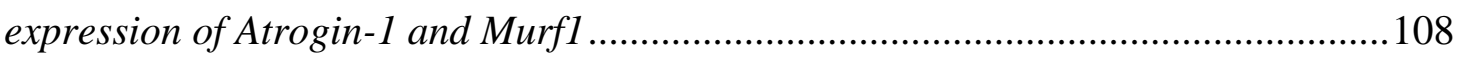

Figure 3.7: Parkin knockdown leads to accumulation of mitochondria in vitro .........110

Figure 3.8: Parkin knockdown alters the levels of mitochondrial fusion and fission

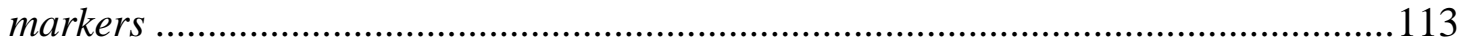

Figure 3.9: Autophagy remains unaltered upon knock down of Parkin ....................115

Figure 3.10: Parkin knockdown leads to mitochondrial dysfunction, increased ROS levels and AMPK $\alpha$ activation in vitro 
Figure 3.11: Parkin knockdown myotubes exhibit a myotube atrophy phenotype in vitro.

Figure 3.12: Loss of Parkin does not lead to activation of FoxO transcription factors

Figure 3.13: Knock down of Parkin increases MURF1 protein levels and activity of the 20S proteaseome 125

Figure 3.14: Parkin knock down does not alter protein synthesis 126

Figure 3.15: Knock out of Parkin results in reduced OXPHOS gene expression, impaired mitochondrial turnover and preservation of the mitochondrial pool in primary myotube cultures

Figure 3.16: Parkin KO mice have reduced levels of mitochondrial Complex I and Complex II proteins and reduced expression of OXPHOS genes in Gas muscle

Figure 3.17: Parkin KO mice display impaired mitochondrial turnover, while autophagy remains unaltered, in muscle.....

Figure 3.18: Knock out of Parkin results in reduced mitochondrial function in primary myotube cultures

Figure 3.19: Four-week-old Parkin KO mice have reduced muscle weights and myofiber size

Figure 3.20: Knock out of Parkin results in moderate but not significant increase in MURF1 protein levels in Gas muscle 


\section{LIST OF TABLES}

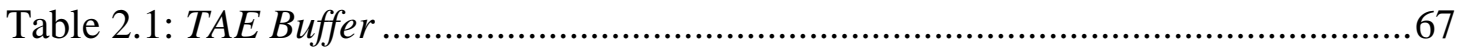

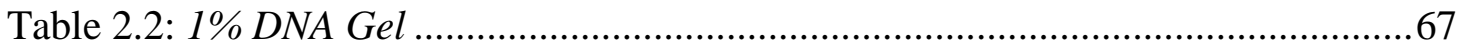

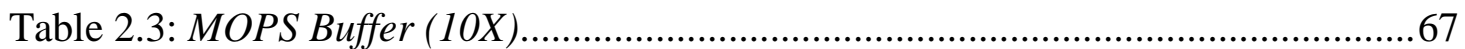

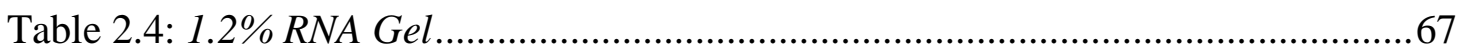

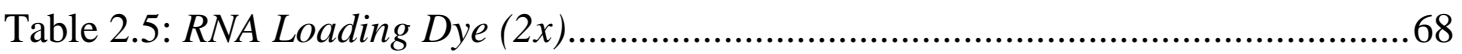

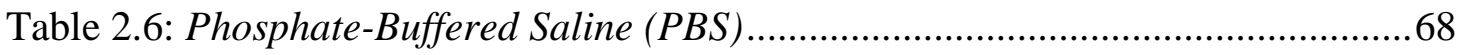

Table 2.7: Radioimmunoprecipitation Assay (RIPA) Buffer.......................................68

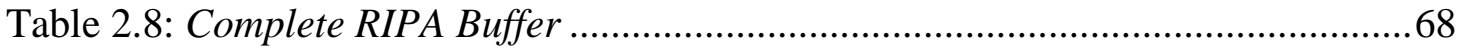

Table 2.9: 16\% Bis-Acrylamide Resolving Gel......................................................69

Table 2.10: 6\% Bis-Acrylamide Stacking Gel ........................................................69

Table 2.11: Protein Loading Dye (4X) for Bis-Tris Gel .............................................69

Table 2.12: Protein Loading Dye (2X) for Tris-Glycine Gel .......................................69

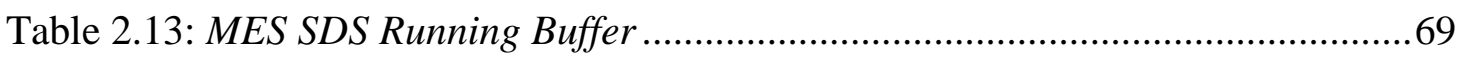

Table 2.14: Tris-Glycine SDS Running Buffer...........................................................69

Table 2.15: Western Blot Wet Transfer Buffer........................................................ 70

Table 2.16: Tris-Buffered Saline with Tween-20 (TBS-T) .........................................70

Table 2.17: Polyvinylpyrrolidone (PVP) Blocking Solution ........................................70

Table 2.18: 5\% Milk Blocking Solution .......................................................................... 70

Table 2.19: 5\% Bovine Serum Albumin (BSA) Blocking Solution ................................70

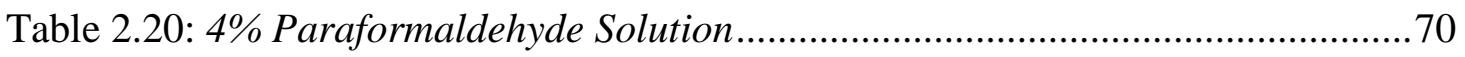

Table 2.21: 2\% Paraformaldehyde Solution ................................................................ 71

Table 2.22: 20:2:1 Fixative Solution for H\&E Staining .............................................71

Table 2.23: Scott's Tap Water .......................................................................................

Table 2.24: Seahorse Assay Medium for MitoStress Test Kit ....................................71

Table 2.25: Seahorse Fatty Acid Oxidation (FAO) Assay Substrate-Limited Medium 72

Table 2.26: Seahorse FAO Assay Medium (KHB Medium)........................................72

Table 2.27: Seahorse FAO Assay Palmitate-BSA Solution ..........................................72

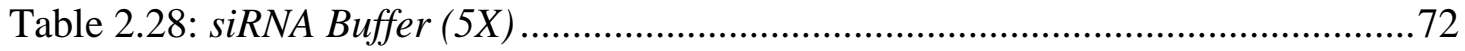

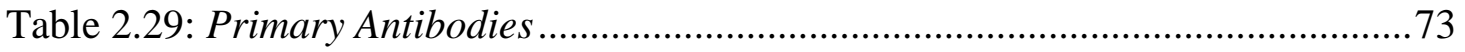

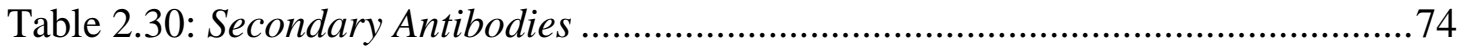

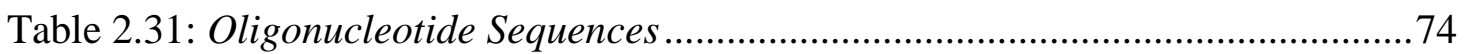

Table 2.32: SMARTpool siRNA Sequences ............................................................76 
Table 2.33: List of Chemicals Used in This Thesis. .76

Table 2.34: List of Reagents Used in This The sis .......................................................78

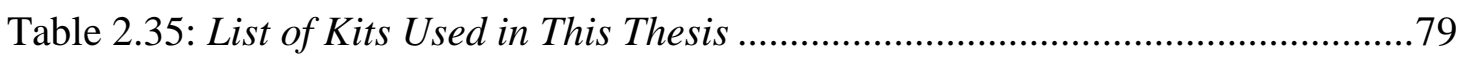

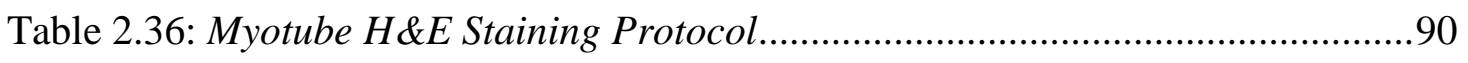

Table 2.37: H\&E Staining Protocol of Muscle Sections..............................................91

Table 3.1: KEGG pathway analysis of differentially expressed genes in Parkin KO

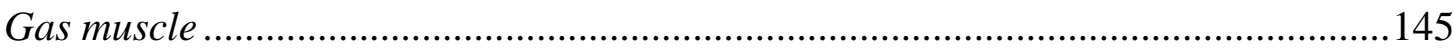

Table 3.2: Genes involved in the ubiquitin proteasome system are upregulated in Parkin KO Gas muscle... 


\section{ABBREVIATIONS}

4EBP1:

$4 \mathrm{E}$ binding protein 1

AAV:

Adeno-associated virus

ADP:

Adenosine diphosphate

AICAR:

5-aminoimidazole-4-carboxamide ribonucleotide

AIDS:

Acquired immune deficiency syndrome

AKT:

Protein kinase B

AMP:

Adenosine monophosphate

AMPK:

5' AMP-activated protein kinase

Ant/Rot:

Antimycin A/Rotenone

Apaf-1:

Apoptotic protease activating factor 1

APC/C:

Anaphase promoting complex/cyclosome

ATG:

Autophagy-related gene

ATP:

Adenosine triphosphate

Atp5:

ATP synthase subunit 5

Atp6:

ATP synthase subunit 6

atrogene:

Atrophy-related gene

bHLH:

Basic helix-loop-helix

BAK:

BCL-2 antagonist/killer 1

BAX:

BCL-2 associated X protein

BCL-2:

B-cell lymphoma 2

BSA:

Bovine serum albumin

$\mathrm{Ca}^{2+}$ :

Calcium ion

$\mathrm{CaCl}_{2}$ :

Calcium chloride

CCCP:

Carbonyl cyanide m-chlorophenyl hydrazine

$\mathrm{CDH} 1$ :

Cadherin 1

CDC20:

Cell division cycle 20

CEE:

Chick embryo extract

CHOP:

C/EBP homologous protein

CMA:

Chaperone-mediated autophagy

CoI:

Cytochrome c oxidase subunit 1

COPD:

Chronic obstructive pulmonary disease

Cox4:

Cytochrome c oxidase subunit 4 
CQ:

CSA:

Cyt $c$ :

Cytb:

DAVID:

DMD:

DMEM:

DML:

DMSO:

DNA:

DRP1:

DUB:

EDL:

EDTA:

eIF2 $\alpha$ :

ER:

ETC:

FACS:

FAO:

Fbx122:

FCCP:

FGF:

FIS1:

FoxO:

GAPDH:

Gas:

GPX:

GR:

GRX:

GWAS:

h:

H\&E:
Chloroquine

Cross-sectional area

Cytochrome c

Cytochrome b

Database for Annotation, Visualization and Integrated

Discovery

Duchenne muscular dystrophy

Dulbecco's modified eagle medium

Dorsomedial

Dimethyl sulfoxide

Deoxyribonucleic acid

Dynamic related protein 1

Deubiquitinating enzyme

M. extensor digitorum longus

Ethylenediaminetetraacetic acid

Eukaryotic translation initiation factor 2-alpha

Endoplasmic reticulum

Electron transport chain

Fluorescence-activated cell sorting

Fatty acid oxidation

F-box and leucine rich repeat 22

Carbonyl cyanide-4-(trifluoromethoxy)phenylhydrazone

Fibroblast growth factor

Fission 1

Fork-head box-O

Glyceraldehyde 3-phosphate dehydrogenase

M. gastrocnemius

Glutathione peroxidase

Glucocorticoid receptor

Glutaredoxin

Genome-wide association study

hour

Hematoxylin and eosin 
$\mathrm{H}_{2}$ DCFDA:

$\mathrm{H}_{2} \mathrm{O}_{2}$ :

HFD:

HGF:

HPG:

HRP:

HSP60:

IBR:

IFM:

IGF:

IMF:

IRS1:

$\mathrm{KCl}$ :

$\mathrm{kDa}$ :

KEGG:

LC3-I:

LC3-II:

LC3:

LPS:

MAMs:

MDV:

Mff:

MFI:

MFN:

$\mathrm{MgCl}_{2}$ :

MIM:

$\mathrm{ml}$ :

$\mathrm{mM}$ :

MOM:

MPC:

MPTP:

MRF:

mtDNA:

mTOR: 2',7'-dichlorodihydrofluorescein diacetate

Hydrogen peroxide

High fat diet

Hepatocyte growth factor

L-Homopropargylglycine

Horse radish peroxidase

Heat shock protein 60

In-between-RING

Indirect flight muscle

Insulin growth factor

Intermyofibrillar

Insulin receptor 1

Potassium chloride

kilo dalton

Kyoto Encyclopedia of Genes and Genomes

Nonlipidated LC3

Lipidated LC3

Microtubule-associated protein 1A/1B-light chain 3

Lipopolysaccharides

Mitochondria-associated membranes

Mitochondria-derived vesicles

Mitochondrial fission factor

Mean fluorescence intensity

Mitofusin

Magnesium chloride

Mitochondrial inner membrane

millilitre

millimolar

Mitochondrial outer membrane

Muscle progenitor cell

1-methyl-4-phenyl-1,2,3,6-tetrahydropyridine

Myogenic regulatory factor

Mitochondrial DNA

Mammalian target of rapamycin 
MuRF1:

MyHC:

MyLC:

$\mathrm{Na}_{3} \mathrm{VO}_{4}$ :

$\mathrm{NaCl}$ :

$\mathrm{NaF}$ :

$\mathrm{NaOH}$ :

Nd1:

$\mathrm{Nd} 2$ :

Nd4:

Nd4l:

Nd6:

Ndufa9:

Ndufb6:

Ndufs3:

Nedd4:

NF- $\kappa B:$

NRF:

nuDNA:

OCR:

OCT:

Oligo:

OPA1:

OXPHOS:

p-:

p62:

PANTHER:

PARIS:

Parkin KO:

PARL:

Pax:

PBS:

PD:
Muscle RING finger protein 1

Myosin heavy chain

Myosin light chain

Sodium orthovanadate

Sodium chloride

Sodium floride

Sodium hydroxide

$\mathrm{NADH}$-ubiquinone oxidoreductase chain 1

NADH dehydrogenase subunit 2

NADH dehydrogenase subunit 4

NADH-ubiquinone oxidoreductase chain $4 \mathrm{~L}$

NADH-ubiquinone oxidoreductase chain 6

NADH-ubiquinone oxidoreductase subunit A9

NADH-ubiquinone oxidoreductase subunit B6

NADH-ubiquinone oxidoreductase core subunit S3

Neuronal precursor cell-expressed developmentally

downregulated 4

Nuclear factor-kappaB

Nuclear respiratory factor

Nuclear DNA

Oxygen consumption rate

Optimum cutting temperature

Oligomycin

Optic atrophy 1

Oxidative phosphorylation

phosphorylated

Nucleoporin p62

Protein Analysis Through Evolutionary Relationships

PARKIN-interacting substrate

Parkin knock out

Presenilins-associated rhomboid-like protein

Paired-box

Phosphate buffered saline

Parkinson's disease 
PEG:

PERK:

PGC-1 $\alpha:$ P

PINK1:

Plk1:

PMSF:

POLG:

PPAR- $\gamma$ :

Ppia:

proteostasis:

PVP:

Q:

$\mathrm{QH}_{2}$ :

Quad:

RIPA:

RLU:

RNA:

ROS:

s.e.m.:

SDH:

Sdha:

SDS:

SiRNA:

SMART:

SNc:

SOD:

Sol:

SS:

SSM:

Suc-LLVY-aminoluciferin

TA:

TEMED:

Tfam:

TGF- $\beta$ :
Polyethylene glycol

Protein kinase RNA-like endoplasmic reticulum kinase PAR- $\gamma$ coactivator- 1 alpha

PTEN-induced kinase 1

Polo-like kinase 1

Phenylmethane sulfonyl fluoride

DNA polymerase gamma

Peroxisome proliferator-activated-gamma

Peptidylprolyl isomerase A

Protein homeostasis

Polyvinylpyrrolidone

Ubiquinone

Ubiquinol

M. quadriceps

Radioimmuniprecipitation assay

Relative light units

Ribonucleic acid

Reactive oxygen species

Standard error mean

Succinate dehydrogenase

Succinate dehydrogenase complex subunit A

Sodium dodecyl sulfate

Small interfering RNA

Simple modular architecture research tool

Substantia nigra pars compacta

Superoxide dismutase

M. soleus

Subsarcolemmal

Subsarcolemmal mitochondria

uccinyl-leucine-leucine-valine-tyrosine-aminoluciferin

M. tibialis anterior

Tetramethylethylenediamine

Mitochondrial transcription factor A

Transforming growth factor-beta 
TNFa:

Trim:

tRNA:

TRX:

UBL:

ULK1:

UPS:

Uqcrc1:

Uqcrc2:

Uqcrfs:

VLL:

Z-LRR-aminoluciferin:

Z-nLPnLD-aminoluciferin:

$\beta$-GPA:

$\mu \mathrm{l}$ :

$\mu \mathrm{M}$ :

$\mu \mathrm{m}^{2}$ :
Tumor necrosis factor alpha

The tripartite motif family

Transfer RNA

Thioredoxin

Ubiquitin-like domain

Unc-51 like autophagy activating kinase 1

Ubiquitin proteasome system

Ubiquinol-cytochrome c reductase core protein 1

Ubiquinol-cytochrome c reductase core protein 2

Ubiquinol-cytochrome $\mathrm{c}$ reductase rieske iron-sulfur

polypeptide 1

Ventrolateral

Z-leucine-arginine- arginine-aminoluciferin

Z-norleucine-proline-norleucine-aspartate

aminoluciferin

Beta-guanadinopropionic acid

microliter

micromolar

Square micrometers 


\begin{abstract}
Skeletal muscle is the largest tissue in the body which accounts for up to $40 \%$ of total body weight in humans. Skeletal muscle as a contractile tissue allows for movement during high energy activities, including exercise. As such, mitochondria, which supply energy to skeletal muscle, play a significant role in maintaining skeletal muscle growth and metabolism. Mitochondrial quality is maintained through processes including mitochondrial fusion and fission and mitochondria turnover, which is mediated through a selective type of autophagy, termed mitophagy. Mitochondrial dysfunction and subsequent mitophagy have previously been implicated in the aetiology of diseases that affect skeletal muscle.

Parkinson's Disease is a neurodegenerative disease characterized by tremors, muscle stiffness and muscle weakness. Molecular genetic analysis confirmed that mutations in PARKIN and PINK1 genes, which play major roles in mitochondrial quality control and mitophagy, are frequently associated with Parkinson's Disease. PARKIN is an E3 ubiquitin ligase that translocates to mitochondria during loss of mitochondrial membrane potential to increase mitophagy. Although muscle dysfunction is noted in Parkinson's Disease, little is known about the involvement of PARKIN in the muscle phenotype of Parkinson's Disease.

In this thesis, a novel role for Parkin in regulating skeletal muscle mass, through its primary role in mitochondrial quality control, is described. Initially, C2C12 myotubes were treated with CCCP to recapitulate PINK1/PARKIN-mediated mitophagy. Results reveal that CCCP treatment leads to abnormal mitochondrial morphology, mitochondrial dysfunction and notable mitophagy, which further validates the function of $\mathrm{CCCP}$ in $\mathrm{C} 2 \mathrm{C} 12$ myotubes. In addition to excess mitophagy, $\mathrm{CCCP}$ treatment resulted in pronounced myotube atrophy and degradation of myofibrillar proteins in vitro. Consistent with previous observations made in other muscle wasting conditions, $\mathrm{CCCP}$ results in reduced levels of phosphorylated FOXO3a (p-FOXO3a) protein and a concomitant increase in the expression of the atrophy-related genes Atrogin-1 and Murf1. Collectively, these results suggest that PINK1/PARKIN-mediated mitophagy upon CCCP treatment leads to mitochondrial dysfunction and myotube atrophy in vitro.

Next, the effect of Parkin knock down on mitochondrial function and $\mathrm{C} 2 \mathrm{C} 12$ myotube area was investigated. C2C12 cells were transfected with either non-targeting (control) or Parkin-specific siRNA. Results revealed preservation of mitochondrial
\end{abstract}


mass in Parkin-siRNA transfected myoblasts as compared to control. Also, knock down of Parkin results in mitochondrial dysfunction and reduced levels of critical OXPHOS proteins. Moreover, knock down of Parkin prevented increased expression of mitochondrial fusion and fission markers. Strikingly, knock down of Parkin resulting in myotubular atrophy in vitro. In agreement with this, Parkin knock down resulted in increased levels of the muscle-specific E3 ligase MURF1 and enhanced proteasome activity.

Mitochondrial number and function was further assessed in a Parkin KO mouse model. Results revealed preservation of mitochondrial number, a significant decrease in levels of critical OXPHOS proteins and reduced mitochondrial respiratory capacity in Parkin KO primary cultures. Also, a reduction in the levels of mitochondrial fission markers in Parkin KO Gas muscle and Parkin KO primary cultures, further suggests that loss of Parkin results in impaired mitochondrial turnover both in vivo and in vitro. Moreover, histological analysis revealed a significant reduction in myofiber crosssectional area of M. tibialis anterior muscle of 4-week-old Parkin KO mice, which is consistent with loss of muscle mass. Reduced muscle mass was supported by increased levels of MURF1 protein in Parkin KO muscle.

To identify gene expression changes that may be responsible for the phenotype observed in Parkin KO mice, microarray analysis was performed. Consistent with mitochondrial dysfunction, genes involved in AMPK signalling, which is activated in response to an increase in ADP:ATP ratio, were upregulated in Parkin KO Gas muscle. In addition, increased expression of genes that are involved in the ubiquitin proteasome protein degradation system suggests that increased protein catabolism is responsible for the skeletal muscle atrophy noted in Parkin KO skeletal muscle.

Taken together, these data underscore an important function of Parkin in skeletal muscle. Specifically, maintenance of Parkin expression is critically important for maintaining skeletal muscle mass, through proceeding regulation of mitochondrial quality control. 


\section{SUMMARY}

Parkinson's Disease (PD) is a neurodegenerative disease characterized by tremors, muscle stiffness and muscle weakness. Molecular genetic analysis confirmed that mutations in PARKIN and PINK1 genes, which play major roles in mitochondrial quality control and mitophagy, are frequently associated with PD. Although muscle dysfunction is noted in PD, little is known about the involvement of PARKIN in the muscle phenotype of PD. In this study, results show that the mitochondrial uncoupler CCCP promotes PINK1/PARKIN-mediated mitophagy in myogenic $\mathrm{C} 2 \mathrm{C} 12$ cells. As a result of this excess mitophagy, CCCP treatment of myotubes leads to the development of myotube atrophy in vitro. Surprisingly, siRNA-mediated knock down of Parkin results in accumulation of dysfunctional mitochondria. In addition, knock down of Parkin also led to myotubular atrophy in vitro. Consistent with these in vitro results, Parkin knockout muscles showed impaired mitochondrial function and smaller myofiber area, suggesting that Parkin function is required for post-natal skeletal muscle growth and development. These findings open new perspectives through investigation of PD from skeletal muscle angle, and may lead to discovery of new therapeutic targets for the treatment of PD. 


\section{REVIEW OF LITERATURE}

\subsection{Skeletal Muscle}

\subsubsection{Skeletal Muscle and Muscle Cell Structure}

The largest tissue in the body is skeletal muscle, which accounts for approximately $38 \%$ and $30 \%$ of the total body weight of humans in men and women, respectively ${ }^{1}$. In addition to controlling locomotion of the body, skeletal muscle is essential for maintenance of posture, as well as contraction and protection of internal organs. Skeletal muscle has specialised structures that can generate tension and contract, and it is the cumulative force generated by each muscle cell that is responsible for muscle contraction. These muscle cells or single muscle fibers (myofibers) are long cylindrical shaped structures, which range in length from $1 \mathrm{~mm}$ to $40 \mathrm{~mm}$. Unlike the typical structure of mammalian cells, single muscle fibers are multinucleated, and in fact, up to 100 nuclei can reside within a single cell ${ }^{2}$.

When sections of skeletal muscle are observed under a microscope, they appear as long packaged myofibrillar proteins attached to tendons through the help of a special connective tissue called the epimysium. Myofibrils are rod-shape structures that consist of actin, myosin, titin and other cytoskeletal proteins and each muscle fiber contains many myofibrils ${ }^{3}$. A second layer of connective tissue termed the perimysium encloses each muscle fascicle, which consists of a bundle of many muscle fibers. Also, blood vessels are spread between fascicles to nourish muscle cells (Figure 1.1). Muscle fibers are embedded in sarcoplasm (cytosol) and enclosed by the sarcolemma (plasma membrane). Neuronal stimuli pass across sarcolemma in the form of calcium ions. Special structures called transverse tubules (T-tubules) facilitate the spread of calcium deep into the muscle fibers by invaginating through the sarcoplasmic reticulum (endoplasmic reticulum) ${ }^{1}$. Also, muscle cells have a heterogeneous mitochondrial population composed of subsarcolemmal (SS) mitochondria, located beneath the sarcolemma, and intermyofibrillar (IMF) mitochondria, which are present between myofibrils. These mitochondrial subpopulations exhibit distinct energetic profiles and respond differently to physiological stimuli ${ }^{1}$.

Myofibrils are composed of bundles of long Myosin (thick) and Actin (thin) myofilaments which are organized into repeating units termed sarcomeres. Sarcomeres are the basic contractile unit of skeletal muscle and display a characteristic light and dark banding pattern under electron microscopy ${ }^{3}$ (Figure 1.2). 


\section{Structure of a Skeletal Mu scle}

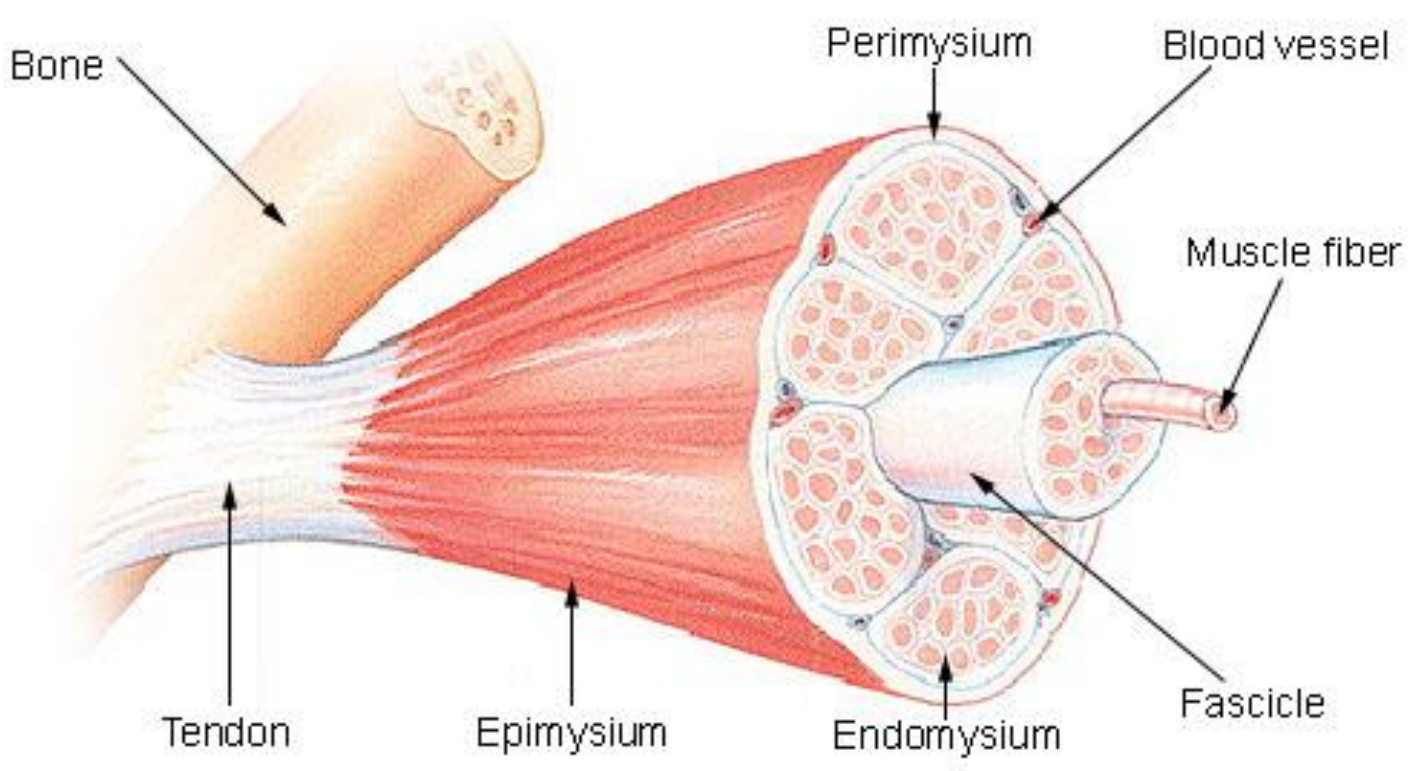

Figure 1.1: Schematic representation of skeletal muscle structure

Single muscle fibers are ensheathed by a layer of connective tissue termed the endomysium. Bundles of muscle fibers, termed fascicles, are further enclosed by a second layer of connective tissue call the perimysium. Blood vessels invaginate through fascicles to provide nutrients and chemicals to each muscle cell. Finally, a third layer of connective tissue, termed the epimysium, encapsulates the entire skeletal muscle, which is connected to bone via tendons. This image was adapted from https://en.wikipedia.org/wiki/Endomysium. (This image is in the public domain. No copyright clearance is required).

Within the sarcomere, light bands alternate with dark bands, which correspond to the I band (isotropic) and A band (anisotropic), respectively. A bands are formed through polymerization of myosin filaments, while I bands lack myosin and are comprised of actin. Actin and myosin filaments overlap with each other, except in the $\mathrm{H}$ zone where only myosin filaments reside. The sarcomere has additional accessory proteins that play an important role in maintaining sarcomeric structure and function. The crosslinking protein $\alpha$-actinin anchors actin filaments to the $\mathrm{Z}$ line. Titin supports myosin filaments and maintains sarcomeric its resting tension, while nebulin contributes to assembly of actin filaments at the $\mathrm{Z}$ line. The unique organization of the sarcomere renders it excitable and contractile and indispensable for generation of movement ${ }^{1}$. 


\subsubsection{Muscle as a Contractile Tissue}

The sliding filament model, which explains how muscle movement occurs at the molecular level was first hypothesized in 1954 by Andrew F. Huxley and Rolf Niedergerke ${ }^{4}$. The latest model, developed by James Spudich and Michael Sheetz, proposes that myosin filaments slide along actin filaments during contraction ${ }^{5}$. During contraction, energy in the form of ATP is used to promote the dissociation of myosin from actin filaments, which are normally tightly attached to myosin in the absence of ATP. Subsequent hydrolysis of ATP into ADP and inorganic phosphate $\left(\mathrm{P}_{i}\right)$ results in a conformational change of the myosin head, which moves the myosin filament to a new position further along the actin filament. Further hydrolysis of ATP rotates the myosin head to its original position, which results in movement of actin filaments toward the center of the sarcomere ${ }^{1,2}$.

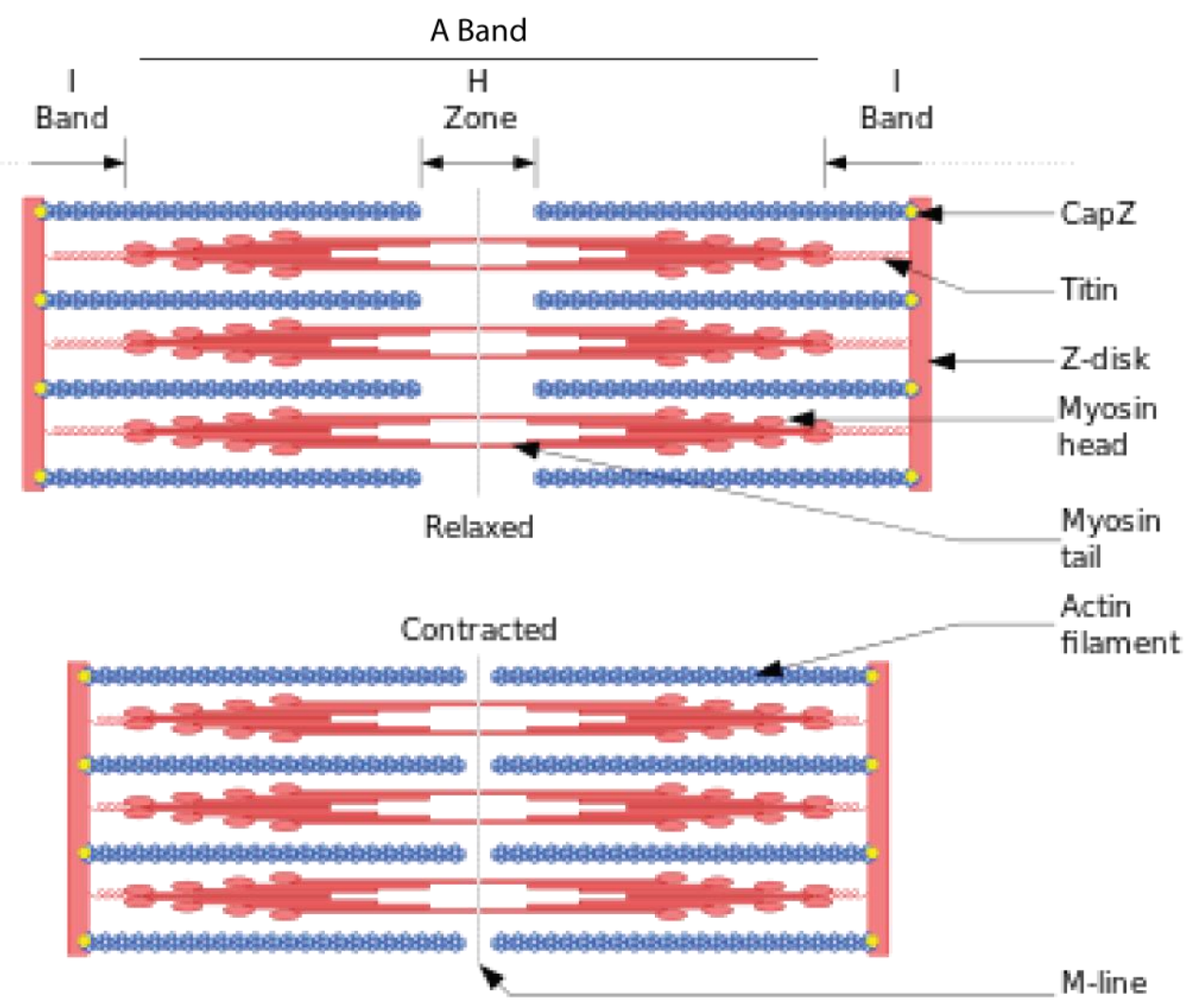

Figure 1.2: Structure of muscle sarcomere

Thick filaments consist of myosin, whereas myofibrillar protein actin forms thin filament. $\mathrm{Z}$ disc limits sarcomere width with $\mathrm{M}$ line at the center. A band is defined by where only thick filaments are observed. I band refer to the region lacking myosin that disappears when contraction reaches maximum. This image was adapted from Wikipedia, https://en.wikipedia.org/wiki/Sarcomere (This image is in the public domain. No copyright clearance is required). 


\subsubsection{The Development of Muscle}

Skeletal muscle development, or myogenesis, is a highly ordered process that is regulated by distinct programmes during prenatal (embryonic), perinatal and postnatal growth ${ }^{6}$.

\subsubsection{Embryonic and Perinatal Myogenesis}

Early developmental signalling initiates the stratification of un-patterned embryos into three germ layers, termed the endoderm, mesoderm and ectoderm. The very first muscle progenitor cells are derived from the mesoderm, which further develops into the paraxial mesoderm, intermediate mesoderm and lateral mesoderm sublayers. Differential expression of critical developmental genes leads to the formation of somites from the paraxial mesoderm ${ }^{7}$. While factors secreted from the lateral plate, neural tube and ectoderm maintain the mesenchymal cell population located at paraxial mesoderm, other signalling factors secreted from the notochord and neural tube promote the migration of cells, through sonic hedgehog (shh) signalling, to form sclerotome at the rostral most site of the embryo ${ }^{8}$. The dorsal part of the somite gives rise to dermomyotome. With the exception of head muscles, skeletal muscles of the body are derived from the dermomyotome ${ }^{9}$. Further signalling cascades results in the polarization of the dermomyotome into dorsomedial (DML) and ventrolateral (VLL) lips, which give rise to deep back musculature and lateral trunk muscle, respectively. Muscle progenitor cells (MPCs), also, reside in dermomyotome. MPCs in the dermomyotome, which is located on the dorsal side of the somite, have high expression of the paired-box transcription factors Pax3 and Pax7 and maintain low expression of the basic helixloop-helix (bHLH) transcription factor $M y f 5^{10,11}$. MPCs in dermomyotome express high levels of certain transcription factors, paired-box-3 (Pax3) and paired-box-7 (Pax7). Later in development, $\operatorname{Pax} 3$ and $\operatorname{Pax} 7$ expressing cells delaminate from the dermomyotome and move to the myotome, which harbours muscle stem cells or satellite cells. Although loss of $\operatorname{Pax} 3$ is lethal in mice, $\operatorname{Pax} 7$-null mice survive into adulthood. However, the satellite cell population is reduced in Pax7-null mice and severe muscle degeneration is noted in adulthood, which in turn results in lethality, suggesting that muscle stem cells that express $\operatorname{Pax} 7$ are required for developmental growth ${ }^{12}$. When satellite cells leave their niche in the myotome, they lose expression of Pax3 and start expressing the myogenic regulatory factor-5 (Myf5). Activated satellite cells coexpressing Pax7 and Myf5 then move to developing limb buds where they undergo serial 
cell division to give rise to myoblasts. However, a unique fraction of these dividing cells may lose expression of myogenic markers and maintain expression of Pax7, which enables the muscle stem cell population to self-renew. Interestingly, cells that maintain self-renewal capacity are deployed between the basal lamina and sarcolemma of myofibers during postnatal myogenesis and become the main pool of reserve muscle stem cells for the adult skeletal muscle system.

Migrating MPCs from the dermomyotome to developing limb buds begin to express high levels of the basic bHLH transcription factors $M y f 5$ and $M y o D^{13}$ and initiate a second wave of myogenesis. In fact, previous studies have revealed that actively dividing myoblasts indeed express $M y f 5$ and $M y o D$, suggesting that both transcription factors play a critical function in cell cycle regulation ${ }^{14}$. The exact mechanisms through which myoblasts align and fuse with each other to form large fully functional muscle tissue remain to be completely delineated (Figure 1.3).

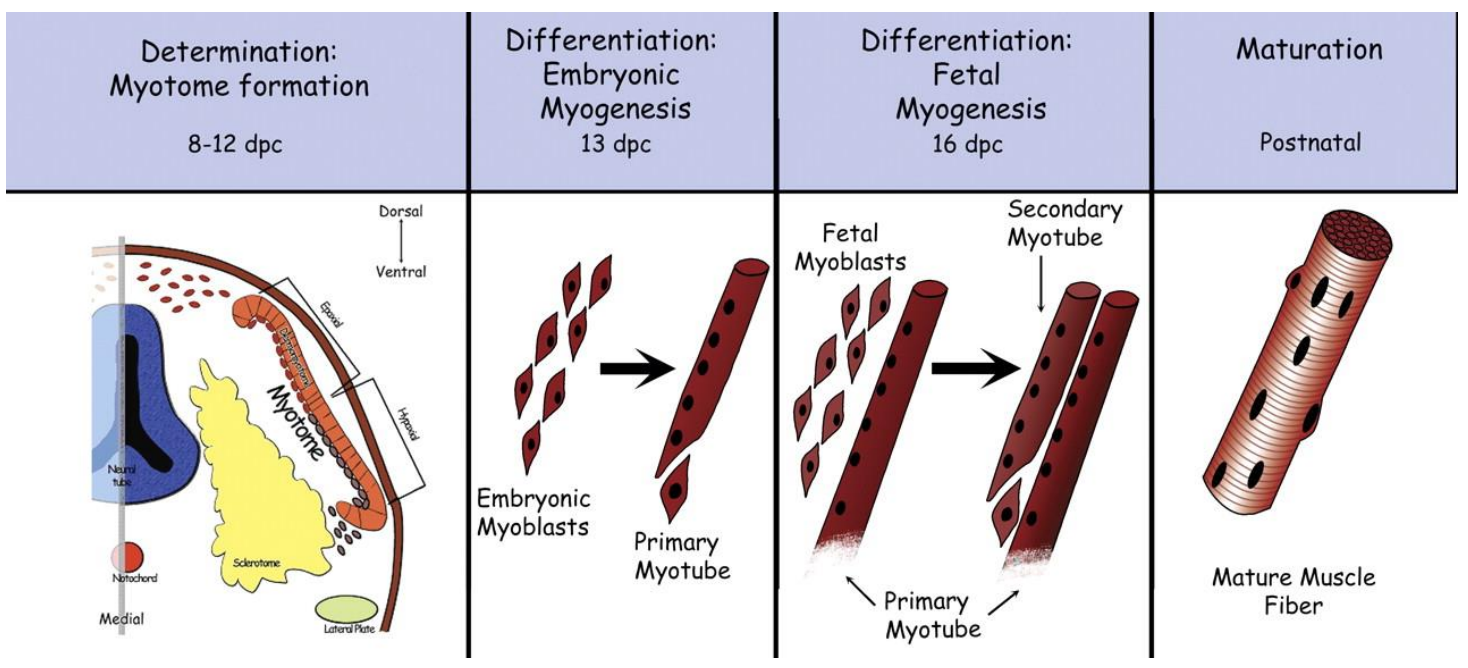

Figure 1.3: Skeletal muscle development

During embryonic development, dermomyotome gives rise to myotome where muscle progenitor cells reside. Embryonic myogenesis starts with fusion of muscle progenitor cells or myoblasts into primary myotubes or primary muscle fibers. At later stages of embryonic development, primary myotubes fuse and form secondary myotubes that surround primary muscle fibers. After birth, primary and secondary muscle fibers fuse and form mature muscle fibers. (Journal of Applied Physiology 104-3, 579-587 (2008) doi:10.1152/japplphysiol.01091.2007) Permission to use this copyrighted image is granted by RightsLink online copy right clearance centre. (See Appendix 6.1) 


\subsubsection{Postnatal Myogenesis and Muscle Regeneration}

Satellite cells are a population of muscle stem cells that are located at the peripheral region of adult muscle fibers, between the basal lamina and plasma membrane of each myofiber, which is known as the satellite cell niche ${ }^{15}$. Postnatal myogenesis and regulation of skeletal muscle mass is primarily attributed to the satellite cell population in skeletal muscle. Satellite cells represents about $2-7 \%$ of all muscle nuclei in adult mice, while at birth it is reported that the number of satellite cells can represent as much as $30 \%$ of total muscle nuclei. Satellite cells are found in quiescent state and express high levels of Pax7 in adult muscle. Upon muscle damage, satellite cells exit quiescence and become activated. Upon activation satellite cells begin to express $M y o D$ along with $\operatorname{Pax} 7$ and proliferate. Previous studies have shown that the growth factors fibroblast growth factor (FGF) and insulin growth factor (IGF) stimulate the proliferation of satellite cells, which underscores the important contribution of growth factors to satellite cell activation ${ }^{16,17}$. Mechanical stretch of muscle, or exercise, can also lead to activation of satellite cells through nitric oxide synthesis and subsequent release of hepatocyte growth factor (HGF) and the secreted glycoprotein, follistatin ${ }^{18}$. Follistatin antagonizes myostatin (reviewed in Section 1.4.4.3), which is a potent negative regulator of myogenesis, suggesting that follistatin may play a role in activation of satellite cells ${ }^{19}$.

The inherent self-renewal capacity of satellite cells enables them to preserve their numbers, which would otherwise be depleted due to environmental damage. It has been shown that satellite cells undergo asymmetric cell division not only to maintain their self-renewal potential but also to give rise to committed myogenic cells ${ }^{20}$. Asymmetric division of satellite cells gives rise to two daughter cells, $\operatorname{Pax} 7-/ M y o D^{+}$ cells, which are committed to differentiation; and $\mathrm{Pax} 7^{+} / M y o D^{-}$cells, which are satellite cells $^{21}$. $\operatorname{Pax}^{+} / \mathrm{MyoD}^{-}$cells reside in the basal lamina, and indicate quiescent and selfrenewed populations of satellite cells. $P a x 7^{-} / M y o D^{+}$cells reside in the myofiber plasma membrane and fuse to form multinucleated myofibers ${ }^{22}$. Interestingly, recent studies have revealed that other mesenchymal cell types, such as bone and brown adipose tissue, can form as a result of asymmetric division of satellite cells ${ }^{23}$. The satellite cell microenvironment maintains the self-renewal capacity of satellite cells and controls commitment of satellite cells into different lineages ${ }^{24}$. Departure of satellite cells from their niche on adult myofibers results in a reduction in their self-renewal capacity, indicating that the satellite cell niche provides extrinsic factors to preserve the satellite 
cell population ${ }^{24}$. Also, it is important that satellite cells reside in close proximity to blood capillaries to be able to readily receive essential nutrients ${ }^{24}$.

Skeletal muscle has the innate ability to repair itself in response to injury. Skeletal muscle regeneration in adult vertebrates is mediated by the activation and migration of satellite cells to the site of injury. In response to injury, satellite cells exit a quiescent state and become active within 24 hours after injury. Activated satellite cells generate myoblasts that either fuse with existing myofibers or fuse with each other to form new myofibers at the site of injury, within two days post injury ${ }^{25}$. Although the mechanism(s) through which muscle trauma activates quiescent satellites cell is not completely understood, it is known that sphingosine-1-phsophate plays a critical role in the entry of satellite cells into the cell cycle, thereby differentiation. In agreement with this, it has been shown that inhibition of sphingosine-1-phsophate halts the process of skeletal muscle regeneration ${ }^{26}$.

Skeletal muscle regeneration is a highly-ordered process. Skeletal muscle trauma stimulates an inflammatory response in muscle fibers. Severe injury results in necrosis of muscle fibers, which secrete factors that promote recruitment of macrophages to the site of injury. Macrophages rapidly proliferate within 24 hours after injury ${ }^{27}$. In addition, migration of macrophages from the epimysium and perimysium towards the site of injury leads to the secretion of several chemoattractants, which in turn recruit neutrophils. Neutrophils proliferate rapidly within a few hours and secrete pro-inflammatory cytokines and proteases that result in degradation of myofibrillar proteins. Also, injury activates satellite cells which in turn give rise to myoblasts migrating to the site of injury. These myoblasts may form new myofibers through fusing with each other or contribute to the existing myofiber population through fusing with existing myofibers. Lastly, damaged muscle tissue is repaired through formation of extracellular matrix around the site of injury. Formation of fibrotic tissue termed as fibrosis, supports the muscle regeneration by acting as scaffold for newly formed myofibers $^{28}$.

\subsubsection{Muscle Fiber Types}

Muscle fibers are classified based on their myofibrillar protein composition and metabolic activity. In 1873, Louis A. Ranvier identified muscle fibers that appeared visibly redder in rabbit skeletal muscle, which contracted slower, when compared to 
paler muscle fibers ${ }^{29}$. This pioneering work led to the classification of muscle fibers into two distinct types. Specifically, Type I fibers (slow-twitch fibers), which are rich in myoglobin, and as such have a characteristic deep red color and Type II fibers (fasttwitch fibers), which appear paler ${ }^{30}$. One of the earliest methods to distinguish muscle fiber types was to perform a succinate dehydrogenase (SDH) activity assay, which is a histochemical staining method that correlates well with mitochondrial content of each muscle fiber ${ }^{31}$. This suggests that muscle fibers exhibit distinct energetic characteristics. Type I fibers, which appear as a dark blue color with SDH staining, are rich in mitochondria, and as such utilize more oxygen for aerobic metabolism, when compared to Type II fibers ${ }^{32}$. Although Type I fibers (slow-twitch fibers) take three times as long as Type II fibers to reach maximum contraction, Type I fibers are able to maintain function during prolonged activities, like standing or endurance exercise ${ }^{33}$. The majority of muscle fibers in the human body are Type II fast-twitch fibers. Unlike slow-twitch fibers, fast-twitch fibers are glycolytic in nature (anaerobic) and are immediately excited, but fatigue faster due to a reduced mitochondrial reservoir ${ }^{34}$.

\subsection{Muscle Wasting in Skeletal Muscle}

\subsubsection{Different Types of Muscle Wasting}

As described above, skeletal muscle has the ability to repair itself in response to injury and trauma. However, under certain situations skeletal muscle tissue is lost, resulting in changes in skeletal muscle flexibility, tension and structure. Skeletal muscle tissue is lost in response to numerous conditions, including prolonged periods of muscle disuse, during muscle aging and in response to chronic illness, which are termed disuse atrophy, sarcopenia and cachexia, respectively. In addition to these conditions, mutations in certain genes may result in skeletal muscle atrophy, for example, mutations in the dystrophin gene are associated with the development of Duchenne-muscular dystrophy (DMD), which is a disease that leads to muscle weakness and muscle atrophy.

\subsubsection{Disuse Atrophy}

A sedentary lifestyle makes muscle weakness inevitable. Lack of physical activity leads to loss of muscle tone and eventually muscle weakness. Previous studies have reported that lack of physical activity, due to the microgravity of spaceflight, results in muscle atrophy in astronauts ${ }^{35}$. Also, conditions that limit physical activity, 
such as immobilization due to casting ${ }^{36}$, spinal cord injury ${ }^{37}$ and prolonged periods of bedrest are directly linked to muscle weakness. Although there are several factors leading to disuse atrophy, reduced anabolic activity and/or increased catabolism appear to be the most robustly altered processes. Notably, immobilized muscle (due to casting) displays increased protein degradation, as opposed to decreased proteins synthesis, in a mouse model, within as little as 4-6 days ${ }^{38}$. In addition to rodents, limb suspension in humans results in a 50\% reduction in global protein synthesis ${ }^{39}$. Moreover, lesions in lower motor neurons can lead to denervation, which leads to loss of muscle activity and subsequent muscle atrophy. In addition, damage to neurons of the spinal cord or the brainstem can cause paralysis, dystonia and eventually muscle atrophy due to denervation of affected muscles ${ }^{40}$.

\subsubsection{Sarcopenia}

The term "Sarcopenia" is derived from the Greek words "sarx" and "penia" meaning flesh and poverty. In 1989, Rosenberg et al. (2011), described Sarcopenia as muscle wasting during aging ${ }^{41}$. Individuals suffering from Sarcopenia lose up to $25 \%$ of their total muscle mass after the age of $45^{42}$. Also, sarcopenia results in additional metabolic changes, such as fat accumulation, fibrosis and insulin resistance, in affected individuals ${ }^{41}$. Besides a noticeable decline in total muscle mass, changes in muscle fiber type have been associated with the progression of sarcopenia ${ }^{43,44}$, with a shift from Type II fibers to Type I fibers reported during sarcopneia ${ }^{45}$. Interestingly, Type II fibers are more susceptible to atrophy due to aging, and as such the cross-sectional area (CSA) of Type II fibers decreases with age ${ }^{46}$.

\subsubsection{Cachexia}

Cachexia refers to the loss of skeletal muscle mass that occurs in response to severe chronic illness, such as AIDS, cancer, COPD, congestive hearth failure and chronic kidney disease (CKD). Individuals suffering from cachexia lose 5\% or more of total body weight over 12 months and suffer from fatigue, muscle weakness and lower appetite $^{47}$. Cachexia diverges from anorexia, as nutritional intervention is not able to overcome the weight loss and loss of skeletal muscle mass noted in these individuals ${ }^{48}$. Cancer cachexia is highly prevalent, with $50 \%$ to $80 \%$ of all cancer patients affected by cachexia $^{49}$. More importantly, almost $20 \%$ of all cancer-related deaths occurs as a result of cachexia as opposed to the direct tumor burden ${ }^{50}$. Cachexia is a multifactorial 
syndrome, yet inflammatory cytokines secreted into the bloodstream, in response to for example tumor formation, play a major role in the development of the cachectic phenotype $^{51}$. Previous studies in cancer patients have underscored the importance of the proinflammatory cytokine, tumor necrosis factor alpha (TNF $\alpha)$ in the progression of cachexia $^{52}$. In vitro experiments have revealed that TNF $\alpha$ is sufficient to induce atrophy through upregulating the muscle-specific E3 ligases Atrogin-1 and Murf1 (Reviewed in Section 1.3.2.2.1), that mediate degradation of myofibrillar proteins ${ }^{53,54}$.

\subsubsection{Muscular Dystrophy}

Muscular dystrophy refers to a group of diseases with hereditary muscle weakness and atrophy, which is associated with mutations in genes that are critical for muscle integrity and function ${ }^{55}$. Duchenne muscular dystrophy (DMD) is the most common form of muscular dystrophy ${ }^{56}$. In 1861, French scientist Guillaume Benjamin Duchenne initially described the disease in detail ${ }^{57}$ and therefore the disease is referred to as, Duchenne muscular dystrophy or DMD. DMD is an X-linked disorder, therefore males are affected more than females, with an incidence of 1 in every 3500 births $^{55}$. Further genetic studies have revealed that most patients carry mutations in the dystrophin gene, which leads to truncation of, or complete loss of, dystrophin protein ${ }^{55}$. The absence of dystrophin protein leads to muscle weakness and increased muscle degeneration. Also, fat accumulation and increased fibrosis have been observed in skeletal muscle of DMD patients. Affected males become wheelchair bound by early adulthood and prematurely die in their early 20s due to failure of cardiac or respiratory musculature function ${ }^{58}$. Dystrophin forms a complex with glycoproteins and functions to anchor the cytoskeleton to the extracellular matrix ${ }^{59}$. Mutations in the dystrophin complex leads to deformation of the cytoskeleton and results in muscle atrophy ${ }^{60}$. Histochemical staining of muscle biopsies from DMD patients has revealed an elevated calcium concentration in the sarcoplasm due to leaky ryanodine receptors (RyR) that allow release of excess $\mathrm{Ca}^{+2}$ from sarcoplasmic reticulum into the sarcolemma. $\mathrm{Ca}^{+2}$ overload in sarcolemma results in activation of calcium-dependent calpains (Reviewed in Section 1.3.2.1.1), which in turn leads to break down of sarcomeric proteins and muscle wasting ${ }^{61}$. 


\subsection{Proteostasis in Skeletal Muscle Mass}

As observed in various type of muscle atrophy, loss of muscle mass results from a shift in the balance of anabolic and catabolic stimuli, whereby protein synthesis is unable to keep up with the accelerated breakdown of myofibrillar proteins ${ }^{62}$. Therefore, understanding protein homeostasis (proteostasis) is crucial to unravelling mechanisms that lead to muscle wasting.

\subsubsection{Protein Synthesis in Muscle Mass Maintenance}

Although loss of muscle mass is mainly regulated by proteolytic pathways, since protein synthesis and degradation pathways share common mediators, the role of protein synthesis in muscle mass maintenance cannot be underestimated. The quality of protein synthesis is controlled by two different processes, namely, translational efficiency, which is defined as the protein translation per unit amount of mRNA and translational capacity, which is limited by ribosomal content of the cell ${ }^{63}$.

\subsubsection{1 mTOR Pathway}

Mammalian target of rapamycin (mTOR) is considered as the main regulator of protein synthesis, which functions through increasing translation efficiency ${ }^{64}$. Moreover, recent studies have shown that rapamycin-mediated inhibition of mTOR suppresses increased ribosomal RNA content observed during hypertrophy, suggesting that mTOR may play a role in regulating not only translation efficiency but also capacity $^{65}$. During hypertrophy, activation of insulin-like growth factor-1 IGF-1 leads to upregulation of Akt/mTOR, which in turn leads to increased protein synthesis ${ }^{66}$ (Figure 1.4). In addition, it was further shown that levels of AKT and mTOR were comparable between wild type and $I g f-1$ overexpressing transgenic mice, suggesting that either additional pathways may play a role in regulating proteins synthesis, or that the Akt/mTOR pathway may have additional partners in regulating protein synthesis, during mechanical loading. Recent studies have shown that the release of membrane bound lipid phosphatidic acid (PA), upon eccentric muscle contraction, leads to activation of mTOR in an IGF-1 independent manner ${ }^{67}$. Moreover, mTOR promotes translation efficiency by phosphorylating, and thereby activating several translation initiation factors, such as ribosomal protein S6 kinase (p70S6K1), 4E-binding protein (4EBP1) and eukaryotic initiation factor 4 (eIF4), to facilitate formation of the $48 \mathrm{~S}$ pre- 
initiation complex ${ }^{68}$. Also, mTOR plays a role in initiation of translation by shuttling eukaryotic initiation factor 2B (eIF2B) to the translation pre-initiation complex, further supporting that mTOR increases global protein synthesis upon its activation ${ }^{69}$.

\subsubsection{2 $\beta$-catenin/c-Myc Pathway}

Besides translational efficiency, translational capacity is modified upon hypertrophy. Previous reports have revealed upregulation of $\beta$-catenin and its downstream target c-myelocytomatosis oncogene $(c-M y c)$, by up to 4- and 3-fold, respectively, in overloaded skeletal muscle, independent of $\mathrm{mTOR}^{70}$. Dephosphorylation of $\beta$-catenin leads to its translocation to the nucleus, which in turn drives expression of $c-M y c$ gene. $c-M y c$ regulates transcription of many genes involved in cell growth, proliferation and apoptosis ${ }^{71}$. Importantly, inhibition of $\beta$-catenin augments hypertrophy observed upon mechanical loading, further suggesting that ribosomal content is increased upon hypertrophy stimulus in a $\beta$-catenin/c-Mycdependent manner ${ }^{70}$.

\subsubsection{Protein Breakdown in Muscle Mass Maintenance}

A decrease in protein synthesis may not necessarily result in loss of muscle mass due to the post-mitotic characteristics of mature myofibers, suggesting that loss of muscle mass is reliant on protein degradation pathways. Several proteolytic systems are present in skeletal muscle, namely the calcium-dependent calpain system, the lysosomal protease system, the ATP-dependent ubiquitin proteasome system and the autophagy pathway, which function together to ensure healthy protein turnover is maintained. The calcium-dependent calpain system, the lysosomal protease system, the ATP-dependent ubiquitin system and autophagy are reviewed in the following sections. 


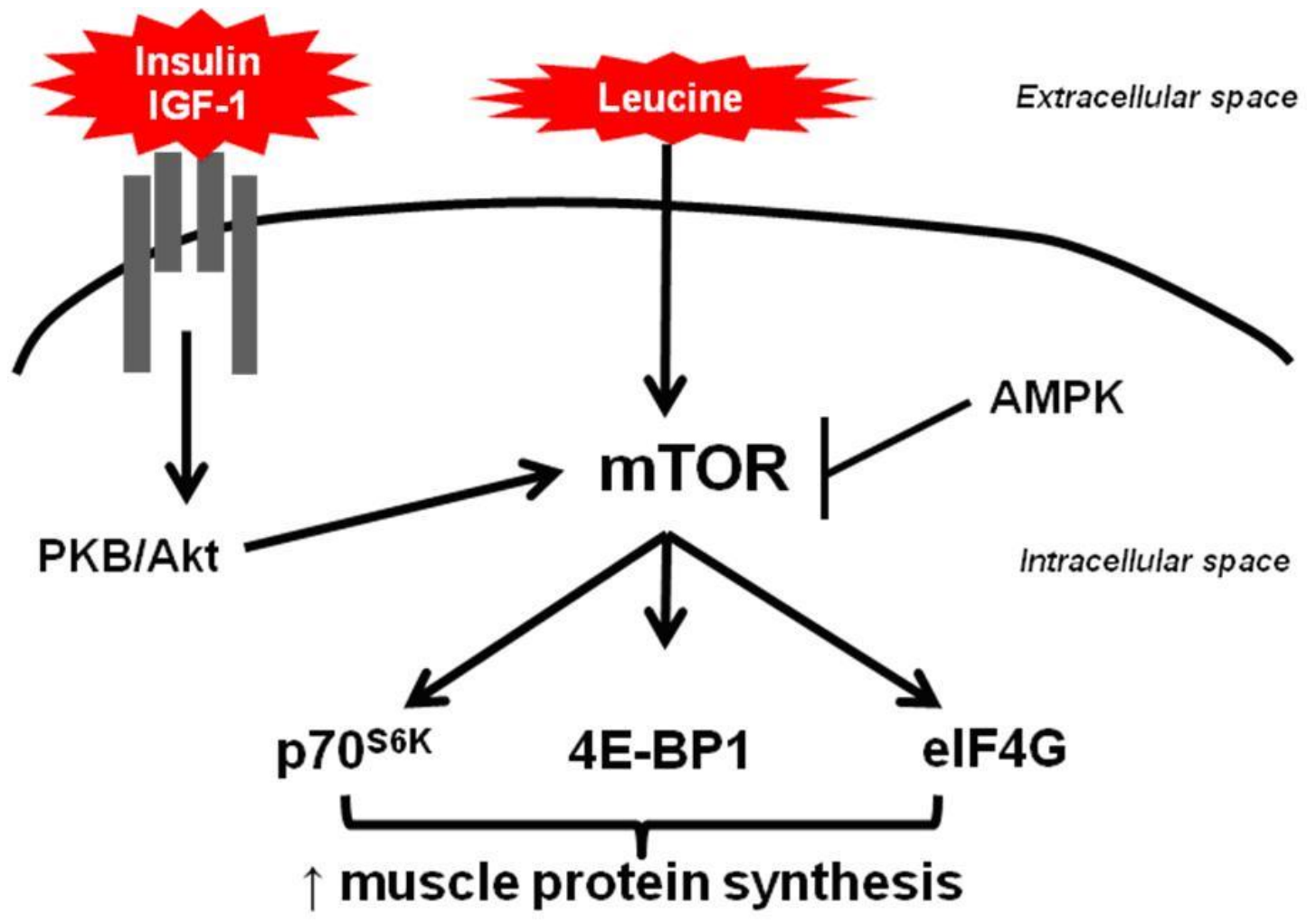

Figure 1.4: mTOR pathway in skeletal muscle protein synthesis

Certain hormones and growth factors (e.g. insulin and IGF-1) lead to activation of mTOR pathway through PKB/Akt. Also, leucine enhances phosphorylation thereby activity of mTOR. In stress conditions, low ATP levels result in activation of AMPK which in turn inhibits activity of mTOR. Activated mTOR promotes protein synthesis through ribosomal protein S6 kinase (p70S6K), 4E-binding protein (4E-BP1) and eukaryotic initiation factor $4 \mathrm{G}$ (eIF4G). (This image is in the public domain and adapted from http://www.efdeportes.com. No copyright clearance is required) 


\subsubsection{Lysosomal Proteases and Calcium Dependent Calpains}

\subsection{Calpains}

Calpains are calcium-dependent cysteine proteinases that are ubiquitously expressed in mammals. Since constant activation of proteolytic mechanisms is detrimental to the cell, calpains remain in an inactive state until their catalytic site is uncapped when $\mathrm{Ca}^{2+}$ levels reach a certain treshold ${ }^{72,73}$. Muscle tissue expresses three isoforms of calpains; calpain 1, 2 and 3, which were detected to be significantly upregulated in response to various muscle wasting conditions ${ }^{74-76}$. Calpains are not able to directly degrade intact myofibrillar proteins but mediate their depolymerization and disassembly. However, calpains may interfere with organization of titin, vimentin and troponin, which are located at Z-disc ${ }^{77}$, leading to their dissociation from the sarcomere, breakdown of sarcomeric structure and release of sarcomeric proteins myosin and actin into the cytosol ${ }^{78}$.

\subsection{Cathepsins}

Lysosomes are highly acidic $(\mathrm{pH}=4-5)$ membrane-bound vesicles that contain various hydrolases namely proteases, phosphatases, lipases and nucleases ${ }^{79}$, as such, lysosomal proteases facilitate the degradation of macromolecules in cells. In addition, lysosomal proteases are implicated in the turnover of cytosolic compartments and organelles. Cathepsins are lysosomal proteases that have been shown to be upregulated during muscle atrophy, although their contribution to the etiology of muscle wasting remains controversial ${ }^{80}$. Importantly, substances that inhibit cathepsins are not able to attenuate myofibrillar protein degradation during muscle wasting, suggesting that cathepsins may not be sufficient to induce muscle atrophy alone ${ }^{81,82}$. Studies have revealed that cathepsins do not participate in breakdown of myofibrillar proteins, but play an important role in turnover of membrane associated proteins such as ligands, transporters and channels, which may explain why cathepsin inhibitors fail to rescue the muscle wasting phenotype ${ }^{83}$.

The lysosomal pathway and ubiquitin proteasome system (reviewed in Section 1.3.2.2) collaborate during muscle atrophy. Ubiquitination, one of the prime posttranslational modifications that qualifies proteins for degradation. Mono- or diubiquitination facilitates degradation of protein substrates through lysosomes, while further ubiquitination is required for activation of the proteasomal pathway ${ }^{84}$. The 
ubiquitin proteasome system is unable to degrade intact myofibrils and in fact relies on the function of the lysosomal pathway, such that disassembled myofibrillar proteins can be subsequently degraded by the ubiquitin proteasome pathway, which will be described in the following section (Section 1.3.2.2).

\subsubsection{Ubiquitin-Proteasome System}

The Ubiquitin proteasome system (UPS) is a multienzymatic system that regulates turnover of proteins involved in biological processes in the cell. The initial step of the UPS begins with activation of ubiquitin, which is followed by sequential ligation of ubiquitin protein to a specific protein substrate and subsequent transfer of the protein substrate to the proteasome.

Proteasome is a giant protein complex where degradation of target protein takes place. is Specifically, E1 enzymes are responsible for activating ubiquitin at its Cterminus through the hydrolysis of $\mathrm{ATP}^{85}$. Subsequently, ubiquitin is then transferred to the cysteine residue of an E2 conjugating enzyme, which conjugates ubiquitin to an E3 ubiquitin ligase, which are responsible for catalyzing the ligation of ubiquitin to the substrate protein. Although there are two E1 enzymes and several E2 enzymes identified to date, a large number of E3 ligases, which is currently estimated to be around 650, exist in mammals, which suggests that E3 ligases have a high level of substrate specificity ${ }^{86,87}$. E3 ligases are classified into three distinct classes; HECT domain E3 ligases, RING-finger E3 ligases and U-box E3 ligases. E3 ligases with a HECT domain harbor two catalytically active domains at their N-terminal and C-terminal. Secific protein substrates bind to the $\mathrm{N}$-terminal HECT domain, whereas the C-terminal HECT domain forms a thioester link with active ubiquitin ${ }^{88}$. RING domain E3 ligases represent the majority of E3 ligases in the mammalian system. Unlike HECT domain E3 ligases, RING domain E3 ligases are not directly involved in ubiquitination, but facilitate the interaction of E2 enzymes with the substrate $\operatorname{protein}^{89}$. U-box E3 ligases represent a newly characterized class of E3 ligase family, with structural and functional similarities to RING domain E3 ligases ${ }^{90}$. 


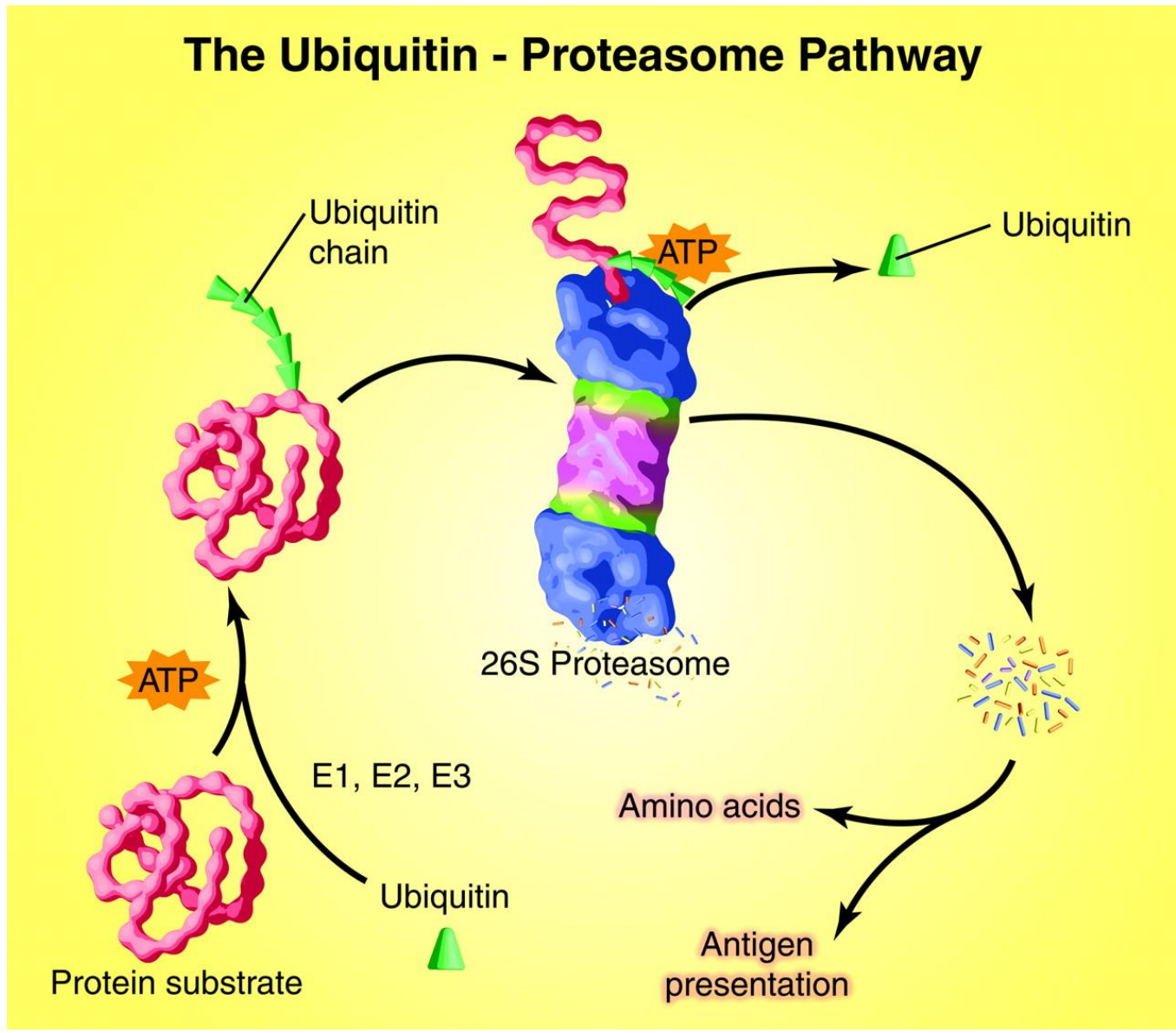

Figure 1.5: Ubiquitin proteasome system machinery

The ubiquitin proteasome system (UPS) is tightly controlled through the sequential action of several enzymes. Initially, an E1 ubiquitin-activating enzyme activates ubiquitin by hydrolysing ATP and transfers activated ubiquitin to the E2 conjugating enzyme. E3 ligases subsequently bind to protein substrates and E2 enzymes, allowing association of ubiquitin with its specific substrate. Multiple rounds of E3-mediated ligation of ubiquitin to target substrate proteins results in their polyubiquitination and subsequent recognition by the proteasome. Polyubiquitination of the substrate, which is reversible via the action of de-ubiquitinating enzyme (USP) activity, results in recognition and degradation of the substrate protein by the proteasome. Digestion of the substrate is further carried out in the cytoplasm through the activity of the peptidases, which in turn results in either turnover of amino acids in the cytosol or sequestration of peptides to the cell membrane to act as antigens. Figure is adapted from the illustration of Josh Gramling - Gramling Medical Illustration, Journal of the American Society of Nephrology, 17: 1807-1819, 2006. doi: 10.1681/ASN.2006010083. Permission to use this copyrighted image is granted by RightsLink online copy right clearance centre (See Appendix 6.2) 
Sequential enzymatic activities of E1, E2 and E3 enzymes leads to the eventual conjugation of ubiquitin to lysine residues in target protein substrates and subsequent recognition of the ubiquitinated substrate by the proteasome. The Proteasome is a multicomplex system that consists of a $19 \mathrm{~S}$ regulatory subunits and a 20S core particle. The $20 \mathrm{~S}$ subunit controls the proteolytic activity of the proteasome, yet it is found in inactive state unless it is activated by the 19S regulatory subunit. Assembly of 19S subunit with the $20 \mathrm{~S}$ core particle allows removal of polyubiquitin chains; resulting in protein unfolding and degradation upon passing of the protein through the barrel-like structure formed by the complex ${ }^{87}$. This process is regulated by ATPases that reside in the 19S regulatory subunit, which function to hydrolyze ATP to provide energy for the process. After entry of substrate protein ito the 20S core complex, which harbors 6 proteolytic sites, target proteins are degraded into peptides that range from 3 to 25 amino acids in length (Figure 1.3). These peptides are released into the cytosol, where they are further digested through the activity of cytosolic peptidases and are subsequently reutilized to synthesize new proteins or as a source of energy.

The expression of E1 enzymes is very low in skeletal muscle and is not affected during muscle atrophy (presumably because it is the common enzyme shared by many processes). In contrast, several E2 conjugating enzymes show differential expression during muscle wasting ${ }^{91}$. Importantly, gene expression analyses, using different muscle wasting models, ranging from cancer cachexia to diabetes mellitus, has revealed a set of common differentially expressed genes that have later been referred to as atrophyrelated genes or "atrogenes"92. Of these genes, two muscle-specific ubiquitin E3 ligases Atrogin-1/Mafbx and Murf1 have been shown to be differentially expressed ${ }^{66,93}$, which are reviewed in the following section (Section 1.3.2.2.1).

\subsection{Atrogin-1/MAFbx and MuRF1}

The E3 ligases Atrogin-1 and Murfl were both identified in a screen for musclespecific E3 ligases associated with several forms of skeletal muscle wasting ${ }^{92}$. Subsequent In vivo studies have revealed that loss of Atrogin-1 and Murfl protect mice from denervation-associated loss of muscle mass ${ }^{66}$. Similarly, Atrogin-1 knockout mice showed resistance to starvation-mediated muscle wasting, whereas knockout of Murf1 restored dexamethasone-induced atrophy in mice ${ }^{94,95}$. ATROGIN-1 has been shown to target the myogenic differentiation protein MyoD and eukaryotic initiation factor-3 
subunit $\mathrm{f}$ (eIF3f), a positive regulator of protein synthesis ${ }^{96,97}$. Studies have shown that MuRF1 seems to facilitate degradation of myofibrillar proteins, such as MyHC, MyLC and troponin ${ }^{98-100}$.

The UPS is challenged by the accumulation of excess unfolded protein aggregates or damaged cytosolic compartments. In fact, protein aggregates and damaged cytosolic compartments impair proteasome function and are as such removed through activation of an alternative response machinery known as autophagy ${ }^{101}$.

\subsubsection{Autophagy}

The word autophagy is derived from combination of Greek words "auto" and "phagein" meaning self-eating. Autophagy is a process that mediates turnover and removal of proteins, cytosolic compartments and organelles in a lysosome-dependent manner ${ }^{102}$. The autophagy-lysosome pathway has gained recent attention, as it has been recently discovered that the autophagy-lysosome system is involved in the development of a vast range of diseases ${ }^{103,104,105}$. Autophagy has been categorized into three different subtypes; chaperone-mediated autophagy (CMA), micro-autophagy and macroautophagy. In CMA, damaged proteins are accompanied by cytosolic chaperones and carried to the lysosomal complex, where damaged proteins are then unfolded and degraded by the lysosomal complex ${ }^{102}$. Micro-autophagy refers to the removal of cytosolic components by the lysosomal membrane directly. However, macro-autophagy refers to the engulfment of cytoplasmic compartments by an intermediary doublemembrane that later fuses with the lysosomal membrane with the help of specific and non-specific receptors ${ }^{106}$. Whether micro-autophagy and CMA take part in muscle wasting is yet to be determined, however, recent studies have emphasized the role of macro-autophagy in muscle atrophy (will be referred as autophagy henceforth).

Glick et al., describes the autophagy process in 5 major steps: 1) formation of a phagophore around the specific target, 2) conjugation of autophagy-related protein 5 (ATG5) to autophagy-related protein 12 (ATG12), 3) recruitment of microtubuleassociated protein 1 light chain 3 (LC3/MAP1LC3) to the phagophore, 4) capture of target cargo by receptor proteins and 5) fusion of the lysosomal membrane with the phagophore and subsequent degradation of target molecules ${ }^{107}$. Early events of autophagy initiates with interaction of autophagy-related protein 1 (ATG1) with autophagy-related protein 13 (ATG13) and autophagy-related protein 17 (ATG17) ${ }^{108}$. The activity of ATG13 is regulated by its phosphorylation status, which is controlled by 
the kinase mTOR, suggesting that phagosome formation is dependent on nutrition and growth factors $^{109}$. Also, it has been shown that ATG5 knock out mice exhibit atrophy phenotype in muscle, presumably due to accumulation of damaged proteins, suggesting that autophagy is required for maintenance of skeletal muscle function and mass ${ }^{110}$. After conjugation of ATG5 to ATG12, non-selective autophagosome engulfment is mainly controlled by the activity of microtubule associated protein light chain 3 beta (LC3B), which is cleaved by autophagy-related protein 4 (ATG4). Cleaved LC3B or LC3-I is activated further in an ATP-dependent manner and a lipid moiety is attached through the action of ATG3, termed lipidation. Since autophagy promotes lipidation of LC3B, the levels of lipidated LC3B (LC3B-II) are typically assessed as a means to monitor the extent of autophagy ${ }^{111}$. While LC3B targets the phagosome (vacuole that contains phagocyted molecules), it needs an adaptor protein on the lysosomal vesicle to allow recruitment of the lysosome to the cargo carrying phagocyted molecules. Ubiquitin-binding protein/Sequestosome 1 (p62/SQSMT1) was the first protein identified to have an adaptor function to allow fusion of the cargo with the lysosome during autophagy ${ }^{112}$. Although previous studies have found that p62/SQSTM1 facilitates degradation of proteins by interacting with LC3B, its role is challenged by a new study that suggests that p62/SQSTM1 is dispensable for selective autophagy ${ }^{112,113}$.

Given that previous works have reported increased autophagy rate in different muscle wasting conditions, including diet-induced muscle wasting ${ }^{114}$, denervation ${ }^{115}$, disuse atrophy ${ }^{116}$, cancer cachexia ${ }^{117}$ and aging ${ }^{118}$, targeting autophagy machinery may have promising therapeutic benefit for muscle atrophy. Interestingly, myofibrillar protein degradation due to overexpression of the transcription factor Atrogin-1, and its upstream regulator forkhead box-O3 (Foxo3), is attenuated by LC3B-siRNA ${ }^{119}$.

\subsubsection{Systemic Mediators of Muscle Wasting}

The sections above have described macro-responses that function to maintain homeostasis during muscle atrophy. The section below describes three systemic hormones and growth factors that have been shown to be activated during muscle atrophy. These systemic factors are; IGF-1, glucocorticoids and myostatin. 


\subsubsection{IGF-1/Akt Pathway}

Insulin-like growth factor-1 (IGF-1) promotes anabolic metabolism in muscle. Previously, ectopic expression of IGF-1 in muscle has been shown to attenuate sarcopenia in old mice and leads to hypertrophy in younger mice ${ }^{120}$. In response to increased levels of IGF-1, the downstream target AKT is phosphorylated and activated. Activation of AKT negatively regulates FOXO transcription factors by phosphorylating them and preventing their nuclear entry. Inhibition of FOXO transcription factors prevents the upregulation of downstream target atrogenes, including Atrogin-1 and Murfl $^{121}$ (Reviewed in Section 1.3.2.2.1). Also, AKT promotes protein synthesis via mTOR signaling ${ }^{122}$ (Reviewed in Section 1.3.1.1). Previous studies have revealed hypertrophy in transgenic mice expressing IGF- $1^{120}$ or AKT ${ }^{123}$ in skeletal muscle, which further support the role of IGF-1/AKT signaling in regulation of skeletal muscle mass through activation of protein synthesis.

\subsubsection{Glucocorticoids}

Glucocorticoids (GCs) are steroid hormones that are widely used to suppress the inflammation associated with chronic inflammatory diseases ${ }^{124}$. GCs bind to glucocorticoid receptors (GR), which in turn dimerizes and translocates to the nucleus where it binds to glucocorticoid response element (GRE). Binding of GR to GRE increases the expression of genes involved in anti-inflammatory response ${ }^{124}$. GCs are found to be in increased in the bloodstream of individuals undergoing skeletal muscle atrophy ${ }^{125,126}$. GCs also have a fiber type-specific effect, with a greater impact noted on Type II fast-twitch fibers, when compared to slow-twitch fibers ${ }^{127}$. Importantly, GCs have been shown to induce protein break down and inhibit protein synthesis in skeletal muscle, which in turn leads to loss of muscle mass ${ }^{128}$. Dexamethasone is a synthetic GC, which is commonly used as immunosuppressant. Mechanistically, Waddell et al., showed that dexamethasone treatment results in increased expression of Murfl through a mechanism involving increased activation of the transcription factor FOXO1. Subsequent loss-of-function experiments by the same group revealed that mutation of the GR prevents dexamethasone-mediated Murfl expression ${ }^{129}$.

\subsubsection{Myostatin}

Myostatin is a member of TGF- $\beta$ superfamily and has been characterized as negative regulatory of muscle growth ${ }^{130}$. Myostatin, as a secreted growth factor, binds 
to a receptor complex on the plasma membrane, which consists of a Type I (Alk4/5) and Type II receptor (ActRIIB) ${ }^{131}$. Interaction of Myostatin with it's Type II receptor leads to a conformational change on the receptor, which in turn facilitates heterodimerization of type II receptor with type I receptor ${ }^{131}$. This interaction triggers the kinase activity of type I receptor, which leads to activation of various downstream signaling pathways, including the canonical Smad signaling pathway ${ }^{132,133}$. Mechanistically, Myostatin has been shown to regulate the growth and differentiation of myoblasts ${ }^{131}{ }^{134}$. Myostatin negatively regulates myoblast proliferation through upregulating $\mathrm{p} 21$, which leads to cell cycle arrest ${ }^{135}$. In addition, myostatin hinders MyoD activation and expression via a Smad3-dependent mechanism, which eventually impedes myogenic differentiation ${ }^{134}$. The importance of myostatin has been revealed in knock out mouse studies, where myostatin-null mice display a remarkable hypertrophy phenotype ${ }^{136}$. Consistent with this phenotype, blockade of myostatin, through injection of either a soluble form of the ActRIIB receptor (sActRIIB) or an antibody raised against myostatin, results in significant skeletal muscle hypertrophy in mice ${ }^{137}$. Excess levels of Myostatin have been shown to promote skeletal muscle wasting. Similar to other muscle wasting stimuli, myostatin inhibits phosphorylation of FOXO1, through suppression AKT, which leads to enhanced activation of FOXO1 and increased expression of atrogenes ${ }^{138,139}$. Importantly, loss of myostatin has been shown to attenuate expression of atrogenes and prolong survival of cancer bearer mice ${ }^{140}$.

\subsection{Mitochondria and Skeletal Muscle}

\subsubsection{Mitochondria and Their Function}

According to the endosymbiotic theory, mitochondria were originally aerobic bacteria that had been taken up into eukaryotic cells $\sim 1.5$ billion years ago ${ }^{141}$. It is further thought that upon this merging, eukaryotic cells became able to exist in oxygen rich environment and utilize oxygen and nutrients to produce energy ${ }^{142}$. Therefore, mitochondria are considered the powerhouse of mammalian cells. In addition to energy production mitochondria have important functions in detoxification of reactive oxygen species and regulating apoptosis. Mitochondria are double membrane structures which consist of outer membrane, inner membrane, intermembrane space and matrix. Outer mitochondrial membrane which encloses entire mitochondria has integral membrane 


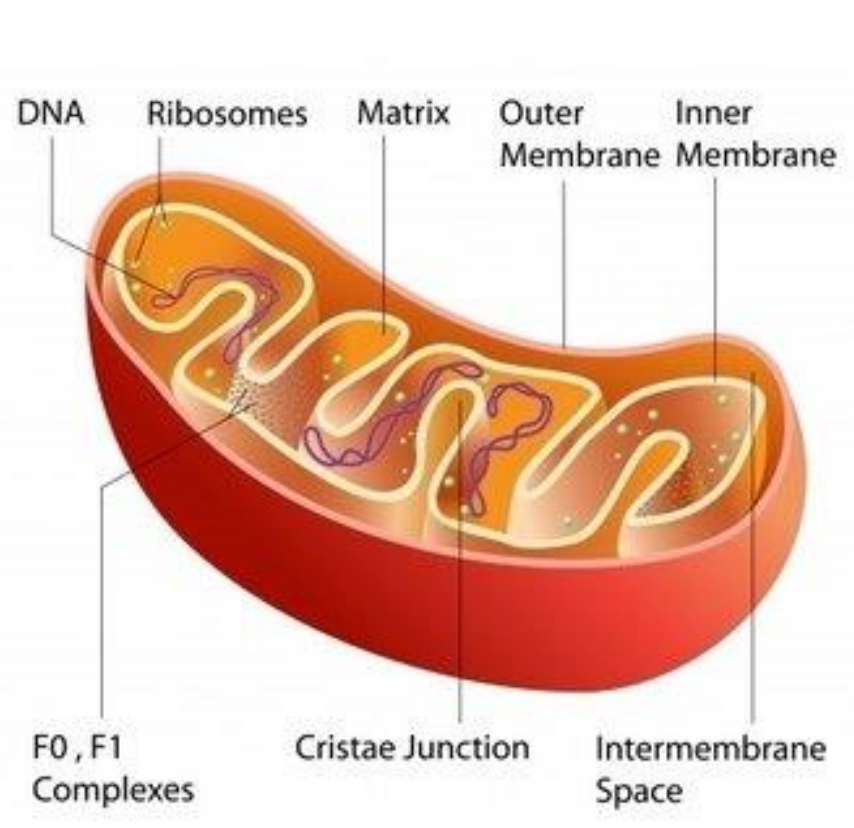

Figure 1.8: Structure of mitochondrion

Mitochondria are double-membrane structures that consist of an outer membrane and inner membrane. Mitochondrial outer and inner membrane are separated by intermembrane space, while mitochondrial inner membrane forms cristae-like structures that invaginate through matrix. Mitochondria have their own DNA and ribosomes that reside in matrix. This image was adapted from Tutorvista, http://biology.tutorvista.com (This image is in the public domain. No copyright clearance is required). proteins which control transport of proteins and molecules across the membrane. Intermembrane space is the space between mitochondrial inner membrane and outer membrane. Inner membrane, which is highly impermeable to proteins, harbors $1 / 5$ of total mitochondrial proteins including critical OXPHOS proteins. Also, inner membrane has highly organized inner cristae that invaginate through the mitochondrial matrix. Such invaginations increase the surface area of the inner mitochondrial membrane, where electron transport chain (ETC) proteins reside, to maximize energy generation. Mitochondrial matrix contains

mitochondrial enzymes, which play a critical role in generation of ATP, mitochondrial ribosomes, tRNAs and mitochondrial DNA suggesting that mitochondrial matrix has a critical role in mitochondrial function ${ }^{143}$. Mitochondria contain their own DNA, which encodes for 22 tRNAs and 13 small peptides that play important roles in oxidative phosphorylation. In the mitochondria, DNA is transcribed by the unique polymerase POLG, accompanied by a mitochondrial-specific single stranded binding protein and a helicase known as TWINKLE ${ }^{144}$.

\subsubsection{Electron Transport Chain and Oxidative Phosphorylation (OXPHOS)}

The mitochondrial outer membrane (MOM) is responsible for controlling the passing of ions and proteins into the mitochondria through pores located on the membrane. Whereas, the mitochondrial inner mitochondria (MIM) is the location where 
electron transport chain ${ }^{145}$ proteins are embedded. The ETC consists of five complexes that orchestrate energy production in eukaryotic cells: complex I (NADH:ubiquinone oxidoreductase), complex II (succinate dehydrogenase), complex III (cytochrome $b c_{1}$ complex), complex IV (cytochrome c oxidase) and complex IV or $\mathrm{F}_{1} \mathrm{~F}_{\mathrm{o}}$-ATP synthase. The ETC is series of enzymatic reactions involving electrons acceptors and donors that eventually results in the production of energy. Electron transport is controlled by two enzymes, coenzyme-Q10, or ubiquinone (Q) and cytochrome c. In the first step of the ETC, NADH enters the ETC cycle at complex I where $\mathrm{Q}$ is reduced to ubiquinol or $\mathrm{QH}_{2}$ through the capture of electrons from the electron carrier, NADH. Complex II balances the electron gradient across the MIM and allows the transport of protons generated by the reduction of NADH by complex I. Electrons carried by $\mathrm{QH}_{2}$ are subsequently passed to cytochrome $c$ in complex III. Reduced cytochrome $c$ is then oxidized at complex IV. This process continues until all electrons are passed to the ultimate electron acceptor, oxygen, which in turn forms water molecules. In response to the sequential donation of electrons through the ETC, a proton gradient is created across the mitochondrial inner membrane ${ }^{146}$. Electron gradient that is formed due to electrons pumped into mitochondrial intermembrane space drives electrons back through the mitochondrial membrane through mitochondrial complex V, ATP synthase. Coupling of electrons through ATP synthase leads to formation of ATP from ADP and inorganic phosphate $\left(\mathrm{P}_{\mathrm{i}}\right)$ in this gigantic protein complex ${ }^{147}$. The complete process of energy generation through the ETC is referred to as oxidative phosphorylation or OXPHOS ${ }^{148}$. However, like every biological system, the ETC is not perfect. Some electrons skip this process and form free radicals through non-enzymatic reactions. The formation of free radicals is potentially deleterious to the cell, which makes energy production by mitochondrial a double-edged sword, as such mitochondrial function needs to be tightly controlled.

\subsubsection{Detoxification of Reactive Oxygen Species (ROS)}

OXPHOS results in the generation of reactive oxygen species (ROS) due to the process of electron capture by mitochondrial complexes. ROS encompasses chemically reactive byproducts containing oxygen, such as hydrogen peroxide $\left(\mathrm{H}_{2} \mathrm{O}_{2}\right)^{149}$, hydroxyl radical $(\mathrm{HO} \bullet)^{150}$ and singlet oxygen $\left({ }^{1} \mathrm{O}_{2}\right)^{151}$. Superoxide $\left(\mathrm{O}_{2}^{-}\right)$is primary and the most abundant precursor of free radicals ${ }^{152}$. Final electron transfer to $\mathrm{O}_{2}$ leads to formation of superoxide that is converted to hydrogen peroxide $\left(\mathrm{H}_{2} \mathrm{O}_{2}\right)$ through the action of two different superoxide dismutases, SOD1 in the inner membrane space and SOD2 in the 
mitochondrial matrix ${ }^{153}$. Excess $\mathrm{H}_{2} \mathrm{O}_{2}$ may leak into the intermembrane space or even cytosol. Since excess $\mathrm{H}_{2} \mathrm{O}_{2}$ leads to oxidative damage of proteins, lipids and mtDNA, mitochondria employ various response mechanisms to prevent deleterious effects of $\mathrm{H}_{2} \mathrm{O}_{2}{ }^{154}$. Oxidized proteins and lipids due to high levels of $\mathrm{H}_{2} \mathrm{O}_{2}$ trigger a repair mechanism in mitochondria that is mediated by glutaredoxin (GRX), thioredoxin 2 (TRX2) and glutathione peroxidase $4(\mathrm{GPX} 4)^{154}$. In addition to these enzymes, antioxidant effects of mitochondrial electron carriers ubiquinol and coenzyme Q (Reviewed in Section 1.4.1.1) have been implicated ${ }^{155,156}$. 


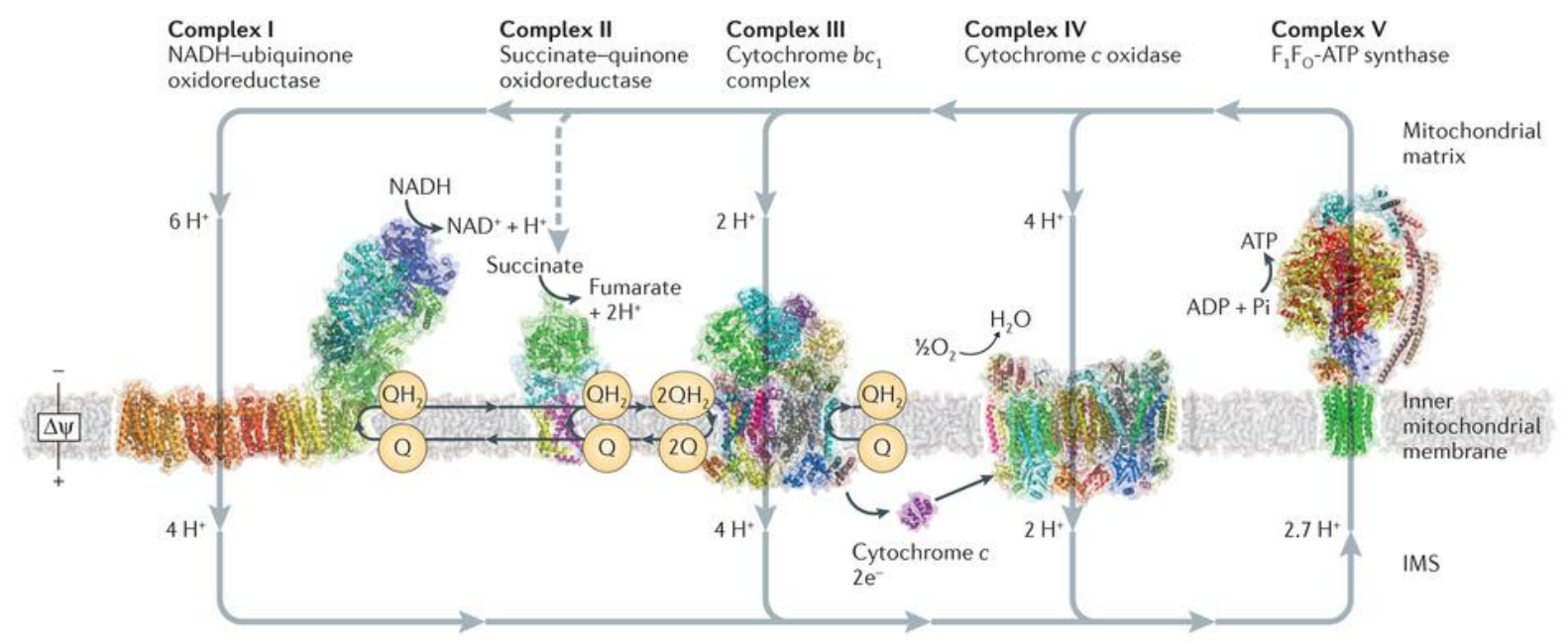

Nature Reviews | Molecular Cell Biology

Figure 1.9: Electron transport chain (ETC) in mitochondrial inner membrane

Electrons captured from NADH enter the system from complex I (NADH-ubiquinone oxidoreductase), which is the largest mitochondrial complex, containing 40 subunits. Complex II (succinate-quinone oxidoreductase) provides an alternative entry site for electrons. Complex I reduces ubiquinone $(\mathrm{Q})$ to ubiquinol $\left(\mathrm{QH}_{2}\right) \cdot \mathrm{QH}_{2}$ is then utilized by complex III (cytochrome $b c_{1}$ complex) to reduce cytochrome $c$, which in turn reduces oxygen at complex IV (cytochrome $c$ oxidase) to form water. 10 protons are pumped across the mitochondrial inner membrane for each NADH molecule oxidized, which generates proton motive force that drives complex $\mathrm{V}, \mathrm{F}_{1} \mathrm{~F}_{0}$-ATP synthase, resulting in ATP formation from ADP and Pi (Nature Reviews Molecular Cell Biology 16, 375-388 (2015) doi:10.1038/nrm3997). Permission to use this copyrighted image is granted by RightsLink online copy right clearance centre (See Appendix 6.3) 


\subsubsection{Mitochondrial Biogenesis}

Given that mitochondria are the major energy production site in the cell, the contribution of mitochondria to cellular biogenesis is undeniable. Studies in mice have identified peroxisome proliferator-activated (PPAR)- $\gamma$ coactivator-1 (PGC-1 $\alpha$ ) as the main regulator of mitochondrial biogenesis ${ }^{157}$. Studies have further demonstrated that PGC$1 \alpha$ regulates mitochondrial biogenesis by activating the nuclear respiratory transcription factors NRF1 and NRF2 ${ }^{158}$. Importantly, these two transcription factors are direct upstream regulators of Tfam, which promotes transcription of mtDNA from two distinct promoter sites ${ }^{159}$. The importance of NRFs to Tfam regulation are clear, as mutations in the NRF binding site of the Tfam promoter, not only hinders binding of NRFs to Tfam, but also reduces Tfam promoter activity ${ }^{160}$. In muscle cells, overexpression of $P g c-1 \alpha$ has been confirmed to increase mitochondrial biogenesis, as assessed by uncoupling protein 2 (Ucp2) upregulation, which that is presumably driven by NRF1 binding on Tfam promoter ${ }^{158}$.

Although PGC-1 $\alpha$ is able to control the transcription of several mitochondrial genes that contribute to mitochondrial biogenesis, mitochondria need to be able to crosstalk with cytosolic pathways to be able to respond to the energy status and nutrient availability of the cell. Energy crisis in the cell has been found to be regulated by an AMP-responsive protein, AMP-activated kinase (AMPK) ${ }^{161}$. In an unstressed state, ATP:ADP ratio remains high due to active catabolic processes. High ATP:ADP ratio drives adenylate kinase to synthesize more ADP which results in a low AMP:ATP ratio, which maintains AMPK in an inactive state. However, in response to stress conditions, such as hypoxia, inadequate carbon sources or exposure to metabolic inhibitors, AMPK becomes activated through the lowering of the ADP:ATP ratio in the cell ${ }^{162}$. Further examination of AMPK in rodent skeletal muscle has revealed that feeding mice with a $\beta$-guanadinopropionic acid ( $\beta$-GPA) diet (which mimics exercise training) not only leads to activation of AMPK, but also increased mitochondrial biogenesis through PGC$1 \alpha$ and NRFs ${ }^{163}$. In addition, studies have revealed increased activity of mitochondrial enzymes citrate synthase, malate dehydrogenese and cytochrome $c$ upon 5aminoimidazole-4-carboxamide-1-romeribofuranoside (AICAR)-induced AMPK activation in rats ${ }^{164}$. Activation of AMPK has also been shown to be responsible for the development of muscle atrophy during hind limb unloading ${ }^{165}$. In addition, sustained activation of AMPK in slow twitch fibers results in atrophy that is associated with a 
sarcopenia-like phenotype in a rat model ${ }^{166}$. Such negative regulation of muscle mass by AMPK could potentially result from its role as an upstream regulator of FOXO transcription factors, as described under Section 1.3.4.1. In fact, a recent study suggests that as a consequence of impaired mitochondrial biogenesis, AMPK is activated and subsequently promotes protein degradation through FOXO transcription factors ${ }^{167}$.

\subsubsection{Apoptosis}

Apoptosis refers to programmed cell death that can be brought about by infection , irreparable damage, severe energy deprivation or DNA damage due to antioxidants ${ }^{168}$. The apoptotic signaling pathway relies on the activity of pro-apototic factors and cysteine proteases, known as caspases, that eventually lead to the release of cytochrome c $(\text { Cyt } c)^{169}$. In healthy conditions, caspase-3/7 and caspase- 9 activity is blocked by XIAP (Inhibitors of Apoptosis), while apoptotic protease activating factor-1 (APAF-1) is inactivated through its auto-inhibitory function ${ }^{170}$. Also, anti-apoptotic factor B-cell lymphoma-2 (BCL-2) inhibits activity of BCL-2 associated X protein (BAX) and BCL2 antagonist/killer 1 (BAK). Upon activation of apoptosis, BCL-2 inhibitory effect is released and thereby, BAX and BAK become activated ${ }^{171}$. Subsequent activation of $\mathrm{BAX}$ and BAK proteins promotes the release of several apoptotic agents, mainly Cyt $c$, Omi/HtrA2 and Smac/DIABLO ${ }^{172}$. In addition, active Cyt $c$ in the cytosol initiates apoptosome formation by interacting with APAF-1, which in turn results in caspase activation $^{168}$. Apoptosome is a tetrameric protein complex that induces activity of caspases, which leads to systematic degradation of cellular components and thereby, cell death. In skeletal muscle, fructose-induced oxidative stress has been associated with decreased mitochondrial membrane potential, poor mitochondrial biogenesis and eventually apoptosis ${ }^{173}$. Also, mitochondrial dysfunction and subsequent apoptosis has been shown to be responsible for selective cell death in muscles of human and rodent juvenile Parkinsonism models ${ }^{174}$.

\subsubsection{Importance of Mitochondria for Skeletal Muscle}

Muscle is highly dynamic tissue with contractible and excitable properties, as such muscle energy expenditure should be tightly controlled. Since muscle cell ATP consumption can increase 100 -fold during contraction ${ }^{175}$, mitochondria are critical for meeting the energy demand of skeletal muscle. Mitochondria supply ATP not only 
during contraction but also for basal metabolism of skeletal muscle. It is also known that defective mitochondrial activity leads to muscle weakness and exercise intolerance ${ }^{176}$. As described above, muscle wasting can occur in response to either chronic or acute stimuli; while acute muscle wasting predominantly affects slow twitch fibers, chronic muscle wasting targets Type-II fibres ${ }^{177}$. Mitochondria, not only generate the ATP required for contraction of muscle and cell metabolism, but also have a role in cell signaling, ROS production, biogenesis, and apoptosis, as reviewed in Section 1.4.1.2, 1.4.1.3 and 1.4.1.4, respectively. Mitochondria can have specialized functions based on their location within skeletal muscle. Mitochondria residing beneath the sarcolemma, are termed subsarcolemmal mitochondria (SSM); whereas mitochondria located between myofibrils are known as intermyofibrillar mitochondria (IMF) ${ }^{178}$. The different metabolic nature of SSM and IFM allows them to respond distinctively to various conditions. These two mitochondrial subpopulations differ in protein and lipid content, and thereby function. For example, SSM are shown to have more branched cristae, probably enabling these mitochondria to more efficiently produce energy ${ }^{179}$. The close proximity of SSM to nuclei and cell membranes allows SSM to generate a more rapid response to cellular challenges, such as during exercise ${ }^{180}$. IFM in skeletal muscle reside in close proximity to the sarcoplasmic reticulum, which enables the two organelles to crosstalk to maintain $\mathrm{Ca}^{2+}$ homeostasis. Also, previous studies have shown that IFM have greater mitochondrial enzyme activity and respiration, when compared to $\mathrm{SSM}^{181}$. Interestingly, skeletal muscle exposed to prolonged activity or disuse stimulate increased turnover of SSM relative to IFM populations of mitochondria ${ }^{182}$.

\subsubsection{Mitochondrial Quality Control}

Mitochondria form a tubular network throughout the cell that is tightly controlled through the action of various quality control systems. Mitochondrial homeostasis can influence the fate of cells, in fact, the apoptosis pathway is centralized around mitochondrial function. Mitochondrial damage due to unfolded mitochondrial proteins, metabolic stress, and exposure to mitochondrial uncouplers triggers proteolytic systems in mitochondria, which result in degradation of mitochondrial components. The following sections describe different mechanisms that maintain mitochondrial quality. 


\subsubsection{Mitochondrial Proteasome System}

Mitochondria respond to the presence of unfolded proteins, that cannot be refolded by chaperones, through activating either cellular or mitochondrial proteolytic machinery. Also, the presence of unassembled or disassembled mitochondrial complex subunit proteins leads to activation of mitochondrial proteasome system. Mitochondrial proteases that reside in the MIM regulate the unfolded protein response, whereas cytosolic proteases have been shown to contribute to degradation of mitochondrial proteins in an ATP-dependent manner ${ }^{183,184}$ (Figure 1.5). AAA+ (ATPases associated with diverse cellular activities) proteases are mitochondrial proteases that play a central role in mitochondrial quality control through their proteolytic activities. Of these mitochondrial proteases, caseinolytic mitochondrial matrix peptidase chaperone subunit (mtClpXP) and Lon peptidase 1 (LON/PIM1) that are highly conserved from bacteria to eukaryotes mediate degradation of oxidized proteins in the mitochondrial matrix ${ }^{185,186}$. Recent studies have revealed the additional mitochondrial proteases HTRA2/OMI and presenilins-associated rhomboid-like protein (PARL), which are associated with neurodegenerative diseases ${ }^{187,188}$. Although the exact target of HTR2A/OMI remains elusive, mice lacking HTR2A/OMI show accumulation of unfolded proteins in mitochondria that results in mitochondrial dysfunction and neuronal apoptosis 189 .

\subsubsection{Mitochondria Derived Vesicles (MDV)}

Mitochondria derived vesicles (MDVs) are a recently characterized class of mitochondrial quality control. In response to oxidative stress, selective cargo that carries damaged proteins and lipids is delivered to lysosomes for ultimate degradation ${ }^{190}$ (Figure 1.5). Interestingly, it has been recently shown that MDVs require PTENinduced kinase (PINK1) and PARKIN, which are primary regulators of mitophagy ${ }^{191}$. However, contrary to this, increased ROS has been shown to induce the MDV pathway ${ }^{192}$. Results suggest that MDVs preserve mitochondrial quality, through maintaining mitochondrial membrane potential, by selective degradation of proteins, prior to the initiation of mitophagy ${ }^{191}$. 


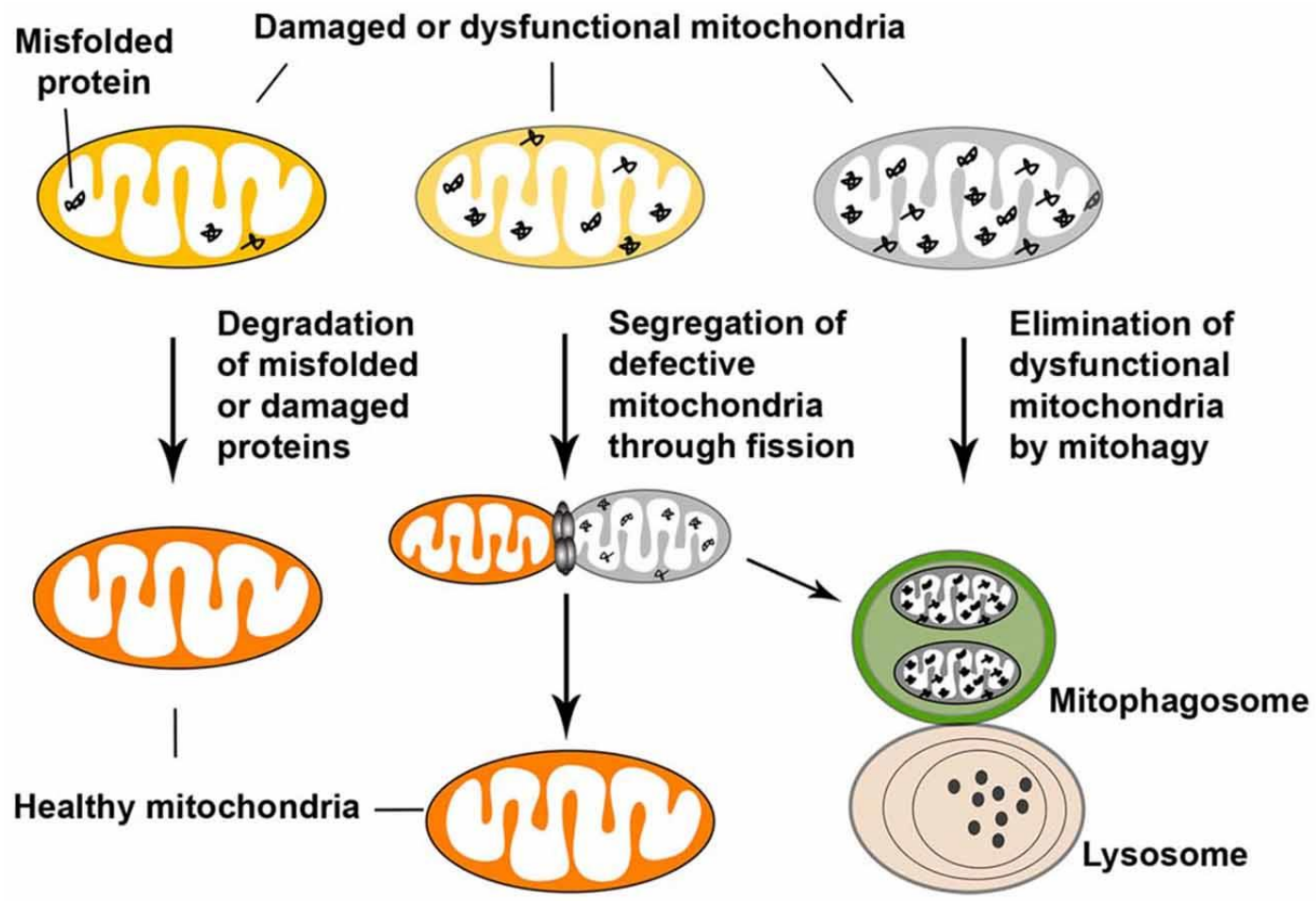

Figure 1.10: Mitochondrial quality control

Unfolded mitochondrial proteins trigger either mitochondrial AAA+ proteases in the intermembrane space and mitochondrial matrix or selective removal of those proteins through fission. If mitochondrial damage overwhelms these two quality control mechanisms, then the entire mitochondrion is isolated from the healthy mitochondrial network and degraded by autophagy machinery (Frontiers in Cellular Neuroscience (2016) 10, 24; doi:10.3389/fncel.2016.00024). Permission to use this copyrighted image is granted by Creative Commons Attribution Licence centre (See Appendix 6.4). 


\subsubsection{Mitophagy}

When mitochondrial damage overwhelms the mitochondrial quality control mechanisms described above, autophagosomes mediate the removal of mitochondria in a systematic and selective manner ${ }^{193,194}$ (Figure 1.6). Mitochondrial autophagy or mitophagy maintains a healthy pool of mitochondria, such that damaged mitochondria are prevented from fusing with healthy mitochondria ${ }^{195}$. Once mitochondria are damaged, several proteins label the mitochondria for recruitment to autophagasomes, enabling the removal of defective mitochondria. In steady-state situations, mitophagy can function to impart beneficial effects on cell development. For example, erythrocytes lose their mitochondria during maturation through mitophagy ${ }^{196}$. Although the reason has not been completely identified, it has been shown that mitophagy-mediated removal of mitochondria in red blood cells helps to keep ROS production in an optimal level ${ }^{197}$. Another example of mitophagy in health occurs in sperm where mitochondria are removed by egg fertilization ${ }^{198}$. Mitophagy is also activated in response to various stress conditions, most notably in the presence of mitochondrial uncouplers ${ }^{199}$, such as carbonyl cyanide m-chlorophenyl hydrazine (CCCP), photo irradiation ${ }^{200}$ and in response to mtDNA deletions ${ }^{201}$. 


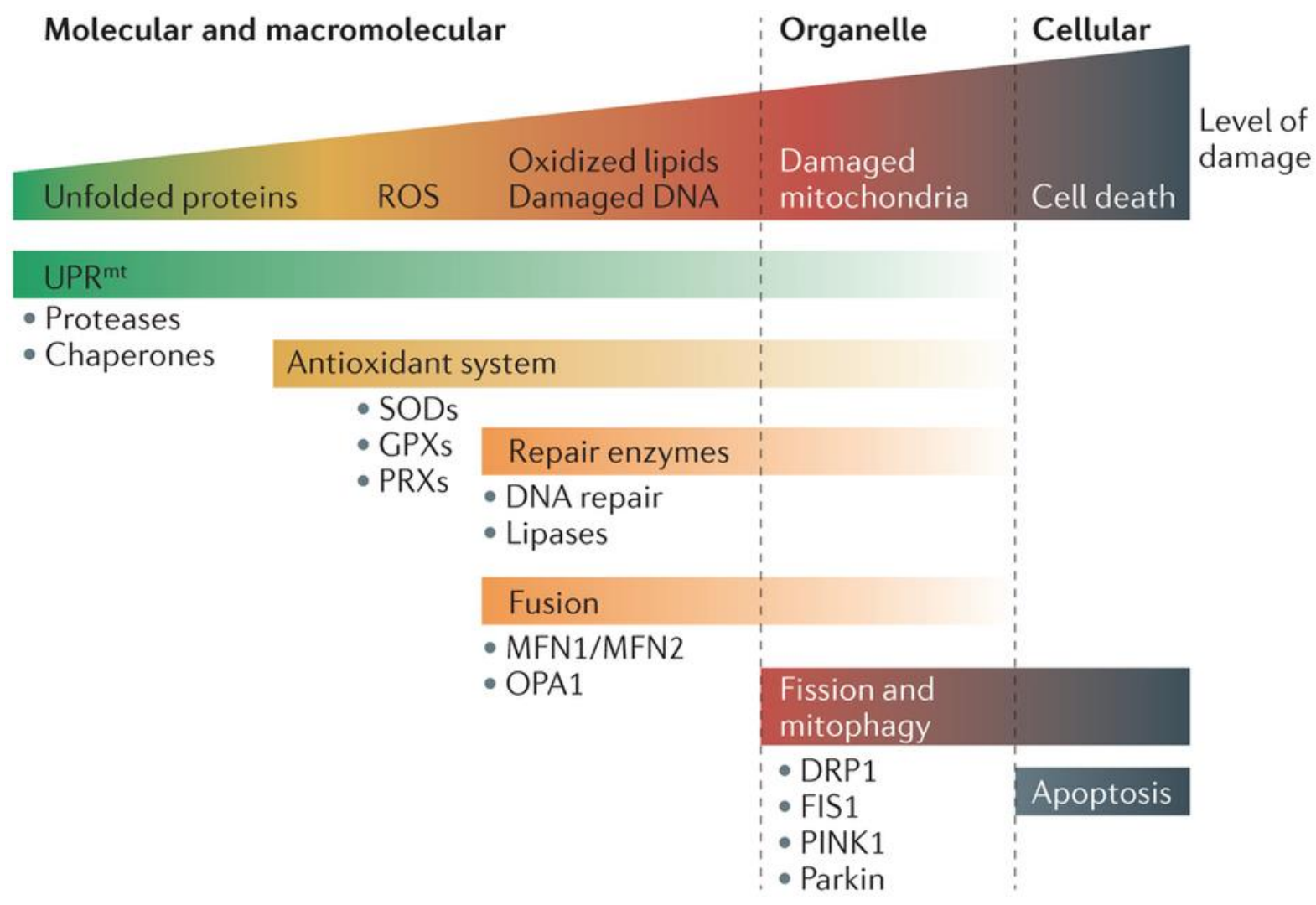

Nature Reviews | Drug Discovery

\section{Figure 1.11: Graphical model of mitochondrial quality control}

Proteostasis is maintained by the mitochondrial ubiquitin proteasome system (UPS ${ }^{\mathrm{mt}}$ ) within the mitochondria. Impaired proteostasis machinery leads to elevated levels of ROS, which turn activates mitochondrial antioxidant enzymes, such as superoxide dismutases (SODs), peroxiredoxins (PRXs) and glutathione peroxidases (GPXs). When excessive ROS overwhelms the detoxification process, damage of mitochondrial DNA (mtDNA), proteins and lipids occurs, which eventually activates DNA repair mechanisms and lipases. If the aforementioned control mechanisms fail to repair the mitochondrial damage, depolarized mitochondria undergo fission and complete removal by autophagsomes through the process termed mitophagy. Persistent failure in repair mechanisms eventually results in cellular apoptosis (Nature Reviews Drug Discovery 12, 465-483 (2013), doi:10.1038/nrd4023). Permission to use this copyrighted image is granted by RightsLink online copy right clearance centre (See Appendix 6.5) 


\subsection{PINK1/PARKIN-Mediated Mitophagy}

The quality of mitochondria is maintained through mitochondrial fusion and fission events. In healthy cells, fusion and fission events are maintained in an equilibrium. However, for engulfment of mitochondrion by autophagosomes, single mitochondrion need to be isolated from the network. Mitochondrial fusion is orchestrated by the highly conserved GTPases, mitofusion protein 1 (MFN1) and 2 (MFN2) in the MOM, while optic atrophy 1 (OPA1) controls mitochondrial fusion events in the $\mathrm{MIM}^{202}$. In addition to mitochondrial fusion there are proteins that regulate mitochondrial fission. Dynamic related protein 1 (DRP1) forms a complex in the mitochondrial outer membrane and facilitates mitochondrial budding with the help of the Drp1 receptor proteins fission 1 (FIS1), mitochondrial fission factor (MFF), mitochondrial dynamics proteins MID49 and MID51 194. Recent studies have consistently reported that mitochondrial depolarization leads to degradation of mitochondrial fusion markers OPA1 ${ }^{161}, \mathrm{MFN}^{162}$ and MFN2 $203-206$.

Mitochondrial quality and mitophagy are primarily controlled by crosstalk between P-TEN-induced kinase (PINK1) and PARKIN (Figure 1.7). In a fly model, similar phenotypes of muscle degeneration, hyperfused mitochondria and impaired mitochondrial function, have been observed upon loss of pinkl and parkin, indicating that these proteins act on the same pathway ${ }^{207}$. Also, previous studies have shown that parkin overexpression partially rescues the phenotype observed upon pinkl knockdown, while pinkl overexpression cannot mask parkin knockdown phenotype, suggesting that PARKIN is downstream target of PINK1 ${ }^{208}$. PINK is a serine/threonine kinase with an $\mathrm{N}$-terminal mitochondrial targeting sequence, serine/threonine kinase domain and a transmembrane helix ${ }^{209}$. PARKIN is a RING-Between-RING (RBR) type E3 ligase with ubiquitin-like domain (UBL) at the N-terminal, 3 RING domains (RING0, RING1, RING2), in-between-RING (IBR) and repressor element of PARKIN (REP) 210 .

Upon mitochondrial depolarization, PINK1 activates PARKIN through phosphorylation of PARKIN at Ser65 ${ }^{211}$. Recently, a new mechanism has been proposed whereby PINK1 phosphorylates ubiquitin to hyperactivate PARKIN catalytic activity $^{212-214}$. According to the proposed model, PINK1 phosphorylation of ubiquitin leads to recruitment of PARKIN to the OMM and further activation of PARKIN catalytic activity. Hyperactivation of PARKIN is achieved by relieving auto-inhibition induced by both the RING0 and UBL domain of PARKIN, which obstruct the catalytic cysteine (Cys431) in the PARKIN RING2 domain and RING1 E2 ligase binding 
domain, respectively ${ }^{213}$. Phosphorylation of PARKIN at Ser65 results in a conformational change in PARKIN structure, thereby releasing repression of the autoinhibitory domain ${ }^{215}$. Mitochondrial membrane associated and activated PARKIN leads to degradation of downstream targets MFN1, MFN2 and several other mitochondrial proteins ${ }^{205,216}$. Activation of PARKIN leads to the activation of two different ubiquitination cascades on mitochondria, namely lysine48 (K48) and lysine63 (K63) ubiquitination. Polyubiquitination at K48 results in degradation of the substrate through proteasome, whereas polyubiquitination at K63 has been reported to be proteasome-independent $^{217}$. Due to preference of autophagy adaptors for K63, autophagic degradation upon K63 ubiquitinated substrates has been implicated ${ }^{218,217,219}$. Ubiquitinated proteins then targeted by an intermediary protein p97/vasolin-containing protein $(\mathrm{VCP})$ for degradation through proteasome ${ }^{220}$. Degradation of mitofusin proteins through the AAA-ATPase activity of $\mathrm{p} 97 / \mathrm{VCP}$ and proteasomal system, and subsequent activation of mitochondrial fission machinery, results in disassembly of unhealthy mitochondria from the healthy mitochondrial pool ${ }^{221}$. Finally, the autophagy adaptors p62/SQSTRM1 and LC3 sequester impaired mitochondria and direct them to autophagosomes for degradation by lysosomal vesicles 222,223 . Importantly, loss of autophagy-related genes has been shown to block mitophagy in CCCP treated cells, underscoring the importance of crosstalk between mitophagy and the canonical autophagy pathway ${ }^{199}$. Although initial studies predicted that p62/SQSTRM1 mediates autophagosome formation around mitochondria, a recent study has shown that it may be dispensable for mitophagy ${ }^{113}$.

Besides mitochondrial turnover, PARKIN takes part in regulating mitochondrial biogenesis. A recent study showed that PARKIN increases proteasomal degradation of PARKIN interacting substrate (PARIS), which represses Pgcla expression, thereby leading to reduced mitochondrial biogenesis ${ }^{224,225}$. Moreover, it is proposed that mitochondrial ubiquitin ligase 1 (MUL1) and PARKIN may work in parallel to promote mitophagy, with both factors converging on MFN2 degradation ${ }^{226}$.

Since mitophagy is an infrequent event in the cell, researchers promote mitophagy through mitochondrial uncouplers. Mitochondrial uncoupling agents have been shown to reduce mitochondrial membrane potential, which in turn results in mitophagy 227 . Although mitochondrial uncouplers are widely used in mitophagy research, they have limitations due to their nonspecific and even detrimental effects in the cell ${ }^{228}$. Rotenone is a parkinsonian toxic which results in a milder reduction in 
mitochondrial membrane potential that is insufficient to induce PINK1/PARKINmediated mitophagy ${ }^{229}$. In addition, other parkinsonian toxins, such as MPP+ and 6hydroxyldopamine (6-OHDA), have been shown to regulate mitophagy through activation of extracellular-signal-regulated protein kinase 2 (ERK2) that is PINK1/PARKIN-independent ${ }^{230,231}$. Due to non-specific effects of uncoupling agents, carbonyl cyanide m-chlorophenyl hydrazine (CCCP) and carbonyl cyanide-p(trifluoromethoxy) phenylhydrazone (FCCP) are most commonly used uncouplers in this context. FCCP and CCCP treatment induce recruitment of PARKIN and LC3 to mitochondria within minutes suggesting that their effect is specific to PINK1/PARKINmediated mitophagy ${ }^{199,232}$. Given that CCCP treatment model has significantly contributed to parkinsonian studies conducted using neurons and HeLA cells ${ }^{113,199,233}$, its effect on skeletal muscle has remained unstudied. Therefore, in this study, myotubes were treated with CCCP to understand the most basic mechanism of PINK1/PARKINmediated mitophagy in C2C12 myotube model. 


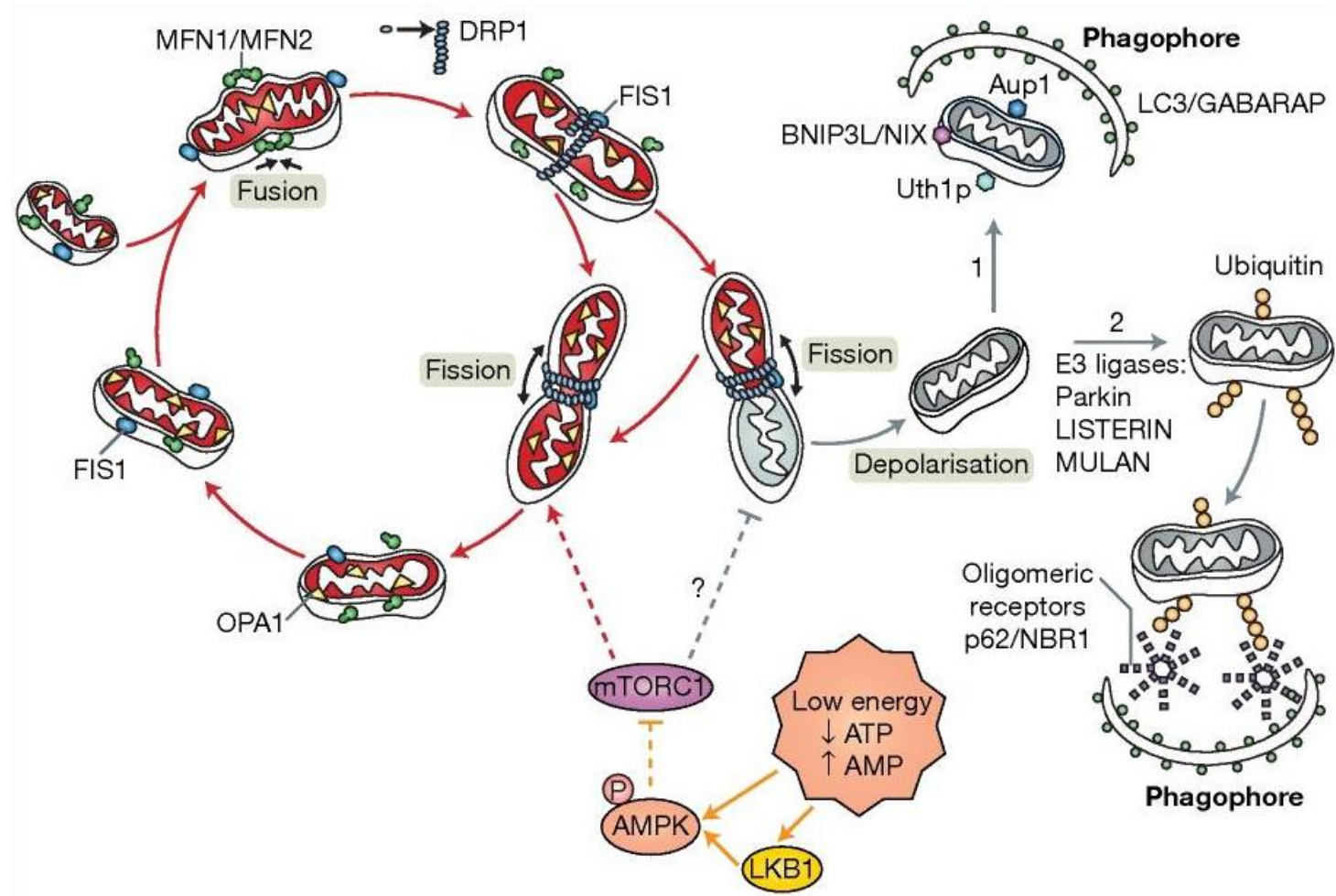

Figure 1.12: Mitochondrial turnover and mitophagy

Mitochondrial fusion is controlled by Mfn2 and Mfn1 while fission is Opa1 and Fis1 dependent. Mitochondrial depolarisation recruits certain E3 ligases to mitochondria. These E3 ligases ubiquitinates specific mitochondrial proteins. Then autophagasomes engulf mitochondria for autophagy. Alternatively, mitochondria may succumb to mitophagy directly through BNIP3L/NIX proteins (Expert Rev Mol Med. 2010 Apr; 12: e12, doi: 10.1017/S1462399410001456). Permission to use this copyrighted image is granted by RightsLink online copy right clearance centre (See Appendix 6.6) 


\subsection{Parkinson's Disease and Mitochondrial Dysfunction}

Parkinson's disease (PD) is the second most common neurodegenerative disease of our era, which is reported to be affecting 7 million people around the world ${ }^{234}$. PD was first characterized by James Parkinson in 1817 in his book "An Essay on the Shaking Palsy". In this initial characterization, PD was described by symptoms including involuntary tremors, muscle weakness, abnormal gait and postural instability. The studies by James Parkinson not only led to clinical recognition of the disease, but also provided a greater understanding of the pathophysiology of PD. Importantly, these initial studies have allowed subsequent studies to be undertaken to decipher the molecular mechanism(s) of PD. In the early 1980s, drug addicts who developed progressive parkinsonism-like phenotype captured wide interest from clinicians and scientists. Studies revealed that the neurotoxic drug 1-methyl-4-phenyl-1,2,3,6tetrahydropyridine (MPTP) leads to Parkinsonian symptoms in these drug users ${ }^{235}$. Further studies revealed that MPTP results in reduced mitochondrial ATP production and elevated ROS levels through inhibiting complex I, suggesting that mitochondrial dysfunction could be a causative factor for $\mathrm{PD}^{236,237}$. Although PD has been thought to be a sporadic disease resulting from neurotoxic agents and additional environmental risk factors $^{238}$, a family history of young individuals affected by PD drove scientists to search for genetic background of this severe neurodegenerative disease. Genetic studies have identified mutations in six genes which are associated with the autosomal recessive form of PD: PARKIN (PARK2), PINK1 (PARK6), DJ-1 (PARK7), SCNA and LRRK2239,240. Interestingly, further studies have revealed that these disease associated genes are involved in mitochondrial quality control and function, which further support the mitochondrial dysfunction theory of PD that was first established following the identification of MPTP-induced parkinsonism ${ }^{235}$.

Mutations in the PARKIN gene are the most common cause of familial PD and in fact, mutation of PARKIN is responsible for almost $80 \%$ of early onset PD ${ }^{241}$. PARKIN mutations were first identified in autosomal recessive juvenile Parkinsonism phenotype observed in Japanese families ${ }^{239}$.Unlike human PD, Parkin knock out mice display reduced locomotor activity when they are placed in a new environment, suggesting a cognitive decline in those mice ${ }^{242}$. In contrast with this study, Perez et al. ${ }^{243}$ have shown no significant alteration in locomotor activity or anxiety-depression-like behaviors in Parkin knock out mice. Previous studies have reported that Parkin knock out mice have impaired dopamine release in substantia nigra and reduced synaptic 
excitability, although nigral degeneration is not as massive as in human PD patients $^{244,245}$.

As mentioned above, mutations in Parkin are linked to mitochondrial dysfunction and development of PD. Interestingly, in the fly model system, knockout of parkin leads to reduced climbing activity and abnormal wing posture, suggesting that flight muscle function is affected upon loss of Parkin ${ }^{174}$. Also, a recent study has documented the presence of a hyperfused mitochondrial network and increased cell death in parkin knock out drosophila ${ }^{226}$. In addition, another study has reported increased sensitivity to mitochondrial toxins and $\beta$-amyloid protein $\mathrm{A} \beta$ in Parkin knock out primary myotubes, which was restored by lentiviral-mediated overexpression of Parkin, suggesting that Parkin has a protective role in skeletal muscle ${ }^{246}$. A further study has revealed that denervation-induced atrophy is alleviated in Parkin knock out mice, which is presumably due to its involvement in autophagy-mediated denervation ${ }^{247}$. In addition to skeletal muscle atrophy, Parkin KO myotubes display reduced insulin sensitivity and impaired respiratory capacity ${ }^{248}$.Consistent with loss of muscle mass and muscle weakness, previous studies have demonstrated that PD patients also have impaired mitochondrial function in muscle tissue, with a reduction in mitochondrial complex activity noted ${ }^{249}$. Although we know that Parkin is central to the mitochondrial quality control mechanism, it remains to be determined whether or not Parkin-mediated mitophagy plays an important role in regulating muscle function and strength. Moreover, no study to date has identified the causative factors responsible for the skeletal muscle atrophy phenotype noted in PD patients and rodent PD models. 


\subsection{Aim of The Project}

$\mathrm{PD}$ is the second most prevalent neurodegenerative disease and affects $1 \%$ of the aging population worldwide ${ }^{250}$. The pathology of PD is characterized by a loss of dopaminergic neurons in substantia nigra pars compacta $(\mathrm{SNc})$ and Lewy bodies in the brains of PD patients ${ }^{251}$. Previous reports have documented reduced mitochondrial complex I and complex IV activity in skeletal muscle of PD patients ${ }^{249,252-256}$. In addition, further studies have attributed impaired motor activity, loss of skeletal muscle mass, muscle weakness, fatigue and resistance to exercise to mitochondrial dysfunction in skeletal muscle of PD patients ${ }^{257-259}$. Changes in muscle mass and function are noted in individuals with PD and that these changes are linked to mitochondrial dysfunction. However, the molecular mechanisms behind the skeletal muscle phenotype in PD is not well understood.

Due to a robust phenotype observed in pinkl and parkin mutants, our current understanding of familial PD has come from studies performed in the drosophila fly model system. In drosophila, loss of parkin results in abnormal wing posture and reduced motor activities, due to a hyperfused mitochondrial network observed in their indirect flight muscles (IFM) ${ }^{174,226}$. A recent study has identified protective role of Parkin in skeletal muscle. Rosen et al., (2006) revealed increased sensitivity of primary myoblasts isolated from newborn Parkin $\mathrm{KO}$ mice to mitochondrial toxins and $\beta$ amyloid protein $A \beta$, which was restored by lentiviral mediated overexpression of Parkin $^{246}$. In addition, it has been shown that Parkin KO mice are resistant to denervation-induced muscle atrophy, presumably due to involvement of Parkin in autophagy-mediated denervation ${ }^{247,260}$. PD is associated with muscle weakness and impaired mitochondrial function in skeletal muscle. Moreover, given that mutations of the PARKIN gene have a causative role in PD, Parkin is important for controlling mitophagy and has a potential role in skeletal muscle, I hypothesize that the E3 ligase Parkin may play a critical role in maintaining skeletal muscle mass and function through regulation of mitochondrial turnover and function. To test this hypothesis the following objectives were undertaken: 


\section{Objectives:}

- Investigate whether CCCP promotes PINK1/PARKIN mediated mitophagy in myoblasts.

- Determine the changes in myotube phenotype induced upon CCCP treatment.

- Assess mitochondrial function and turnover in Parkin knock down and knock out models.

- Define the role of Parkin in regulation of skeletal muscle mass using in vitro and in vivo Parkin loss-of-function models.

- Elucidate potential mechanism(s) responsible for regulation of skeletal muscle mass and function in Parkin knock down and knock out models. 


\section{MATERIALS AND METHODS}

\subsection{Materials}

Materials used in this study have been described in the following section with details of compositions, companies and catalogue numbers provided.

Compositions of all materials used in this thesis is provided in the following section.

\subsubsection{DNA Electrophoresis}

Details of reagents used for DNA gel electrophoresis are provided in the following tables.

Table 2.1: TAE Buffer

$\begin{array}{cc}\text { TAE Buffer } & \text { Concentration/Amount/Volume } \\ \text { Tris-HCl (pH 7.6) } & 40 \mathrm{mM} \\ \text { EDTA } & 1 \mathrm{mM} \\ \text { Acetic Acid } & 20 \mathrm{mM}\end{array}$

Table 2.2: 1\% DNA Gel

$1 \%$ DNA Gel

Ultra Pure Agarose

TAE Buffer

\section{Concentration/Amount/Volume \\ $1 \mathrm{~g}$ \\ $100 \mathrm{ml}$}

\subsubsection{RNA Electrophoresis}

Reagents used for RNA gel electrophoresis are given in the tables below.

Table 2.3: MOPS Buffer (10X)

MOPS Buffer (10X)

MOPS

Sodium Acetate

EDTA
Concentration/Amount/Volume

$200 \mathrm{mM}$

$50 \mathrm{mM}$

$20 \mathrm{mM}$

Table 2.4: 1.2\% RNA Gel

$1 \%$ DNA Gel

Ultra Pure Agarose

MOPS Buffer (1X)

$37 \%$ Formaldehyde

Top up with MQ water to
Concentration/Amount/Volume

$1.2 \mathrm{~g}$

$10 \mathrm{ml}$

$18 \mathrm{ml}$

$100 \mathrm{ml}$ 
Table 2.5: RNA Loading Dye (2x)

$\begin{array}{cc}\text { RNA Loading Dye }(2 \mathrm{X}) & \text { Concentration/Amount/Volume } \\ \text { MOPS (10X) } & 10 \%(\mathrm{v} / \mathrm{v}) \\ \text { Deionised Formaldehyde } & 20 \%(\mathrm{v} / \mathrm{v}) \\ \text { Deionised Formamide } & 50 \%(\mathrm{v} / \mathrm{v}) \\ \text { Bromophenol Blue } & 0.02 \%(\mathrm{w} / \mathrm{v}) \\ \text { Glycerol } & 5 \%(\mathrm{v} / \mathrm{v}) \\ \text { EDTA (pH 8.0) } & 1 \mathrm{mM} \\ \text { Ethidium Bromide } & 40 \mu \mathrm{g} / \mathrm{ml}\end{array}$

\subsubsection{Western Blot Analysis}

The following tables outline details of reagents used for total protein extraction and subsequent western blot analysis to detect protein levels.

Table 2.6: Phosphate-Buffered Saline (PBS)

$\begin{array}{cc}\text { PBS (1X) } & \text { Concentration/Amount/Volume } \\ \text { Oxoid }^{\mathrm{TM}} \text { Phosphate-Buffered Saline Tablet } & 1 \text { tablet } \\ \text { Top up with MQ water to } & 100 \mathrm{ml}\end{array}$

Table 2.7: Radioimmunoprecipitation Assay (RIPA) Buffer

$\begin{array}{cc}\text { RIPA Buffer } & \text { Concentration/Amount/Volume } \\ \text { Nonidet P-40 (NP-40) } & 1 \%(\mathrm{v} / \mathrm{v}) \\ \text { SDS } & 0.1 \%(\mathrm{w} / \mathrm{v}) \\ \text { Sodium Deoxycholate } & 0.5 \%(\mathrm{w} / \mathrm{v}) \\ \text { Sodium Fluoride } & 50 \mathrm{mM}\end{array}$

Top up with PBS to required volume

Table 2.8: Complete RIPA Buffer

$\begin{array}{cc}\text { Complete RIPA Buffer } & \text { Concentration/Amou } \\ \text { RIPA Buffer } & 7 \mathrm{ml} \\ \text { Sodium Orthovanadate } & 1 \mathrm{mM} \\ \text { PMSF } & 1 \mathrm{mM} \\ \text { cOmplete }^{\mathrm{TM}} \text { Mini Protease Inhibitor } & 1 \text { tablet } \\ \text { PhosSTOP }^{\mathrm{TM}} & 4 \text { tablets }\end{array}$


Table 2.9: 16\% Bis-Acrylamide Resolving Gel

$16 \%$ Resolving Gel

$30 \%$ Acrylamide/Bis Stock

$1.5 \mathrm{M}$ Tris- $\mathrm{HCl}(\mathrm{pH} 8.8)$

SDS

Ammonium Persulfate

TEMED

MQ water
Concentration/Amount/Volume

$4.27 \mathrm{ml}$

$2 \mathrm{ml}$

$80 \mu 1$

$80 \mu 1$

$8 \mu 1$

$1.6 \mathrm{ml}$

Table 2.10: 6\% Bis-Acrylamide Stacking Gel

$\begin{array}{cc}\text { 6\% Stacking Gel } & \text { Concentration/Amou } \\ \text { 30\% Acrylamide/Bis Stock } & 1 \mathrm{ml} \\ \text { 0.5M Tris-HCl (pH 6.8) } & 1.25 \mathrm{ml} \\ \text { SDS } & 50 \mu \mathrm{l} \\ \text { Ammonium Persulfate } & 50 \mu \mathrm{l} \\ \text { TEMED } & 5 \mu \mathrm{l} \\ \text { MQ water } & 2.6 \mathrm{ml}\end{array}$

Table 2.11: Protein Loading Dye (4X) for Bis-Tris Gel

Protein Loading Dye for Bis-Tris Gel (4X) Concentration/Amount/Volume

LDS Sample Buffer (4X)

$75 \%(\mathrm{v} / \mathrm{v})$

$\beta$-mercaptoethanol

$25 \%(\mathrm{v} / \mathrm{v})$

Table 2.12: Protein Loading Dye (2X) for Tris-Glycine Gel

Protein Loading Dye For Tris-Glycine Gel $(2 \mathrm{X})$

Concentration/Amount/Volume

Tris-Glycine SDS Sample Buffer (2x)

$90 \%(\mathrm{v} / \mathrm{v})$

$\beta$-mercaptoethanol

$10 \%(\mathrm{v} / \mathrm{v})$

Table 2.13: MES SDS Running Buffer

MES SDS Running Buffer

Concentration/Amount/Volume

NuPAGE ${ }^{\circledR}$ MES SDS Running Buffer

(20X)

$50 \mathrm{ml}$

MQ water

$950 \mathrm{ml}$

Table 2.14: Tris-Glycine SDS Running Buffer

Tris-Glycine SDS Running Buffer

Novex $^{\text {TM }}$ Tris-Glycine SDS Running

Buffer (10X)

MQ water
Concentration/Amount/Volume

$100 \mathrm{ml}$

$900 \mathrm{ml}$ 
Table 2.15: Western Blot Wet Transfer Buffer

$\begin{array}{cc}\text { Transfer Buffer } & \text { Concentration/Amount/Volume } \\ \text { Tris Base } & 25 \mathrm{mM} \\ \text { Glycine } & 192 \mathrm{mM} \\ \text { Methanol } & 20 \%(\mathrm{v} / \mathrm{v})\end{array}$

Top up with MQ water to required volume

Table 2.16: Tris-Buffered Saline with Tween-20 (TBS-T)

$\begin{array}{cc}\text { TBS-T } & \text { Concentration/Amount/Volume } \\ \text { Tris- } \mathrm{HCl}(\mathrm{pH} 7.5) & 20 \mathrm{mM} \\ \mathrm{NaCl} & 150 \mathrm{mM} \\ \text { Tween-20 } & 0.1 \%(\mathrm{v} / \mathrm{v})\end{array}$

Top up with MQ water to required volume

Table 2.17: Polyvinylpyrrolidone (PVP) Blocking Solution

$\begin{array}{cc}\text { PVP Blocking Solution } & \text { Concentration/Amount/Volume } \\ \text { PVP } & 1 \%(\mathrm{w} / \mathrm{v}) \\ \text { Polyethylene Glycol (PEG) } & 1 \%(\mathrm{w} / \mathrm{v}) \\ \text { Bovine Serum Albumin (BSA) } & 0.3 \%(\mathrm{w} / \mathrm{v})\end{array}$

Top up with TBST to required volume

Table 2.18: 5\% Milk Blocking Solution

$\begin{array}{cc}5 \% \text { Milk Blocking Solution } & \text { Concentration/Amount/Volume } \\ \text { Skimmed Milk Powder } & 5 \%(\mathrm{w} / \mathrm{v})\end{array}$

Top up with TBST to required volume

Table 2.19: 5\% Bovine Serum Albumin (BSA) Blocking Solution

$\begin{array}{cc}5 \% \text { BSA Blocking Solution } & \text { Concentration/Amount/Volume } \\ \text { BSA } & 5 \%(\mathrm{w} / \mathrm{v})\end{array}$

Top up with TBST to required volume

\subsubsection{MitoTracker Red CMXRos Confocal Analysis}

Details of the fixatives used for MitoTracker red staining analysis are given below.

Table 2.20: 4\% Paraformaldehyde Solution

$\begin{array}{cc}\text { 4\% Paraformaldehyde } & \text { Concentration/Amount/Volume } \\ \text { Paraformaldehyde } & 40 \mathrm{~g} \\ \mathrm{PBS} & 800 \mathrm{ml} \\ \mathrm{NaOH} & \text { As required to dissolve PFA } \\ \mathrm{HCl} & \text { As required to reach pH } 6.9 \\ \text { Top up with MQ water to } & 1000 \mathrm{ml}\end{array}$


Table 2.21: 2\% Paraformaldehyde Solution

$2 \%$ Paraformaldehyde

Concentration/Amount/Volume

4\% Paraformaldehyde Solution

$250 \mathrm{ml}$

MQ water

$250 \mathrm{ml}$

\subsubsection{Hematoxylin and Eosin (H\&E) Staining}

Details of reagents used for H\&E staining are listed in the tables below.

Table 2.22: 20:2:1 Fixative Solution for H\&E Staining

$\begin{array}{cc}\text { 20:2:1 Fixative } & \text { Concentration/Amount/Volume } \\ 70 \% \text { ethanol } & 20 \mathrm{ml} \\ 37 \% \text { Formaldehyde } & 2 \mathrm{ml} \\ \text { Acetic Acid } & 1 \mathrm{ml}\end{array}$

Table 2.23: Scott's Tap Water

$\begin{array}{cc}\text { Scott's Tap Water } & \text { Concentration/Amo } \\ \text { Sodium Bicarbonate } & 3.5 \mathrm{~g} \\ \text { Magnesium Sulphate } & 20 \mathrm{~g} \\ \text { MQ Water } & 1000 \mathrm{ml}\end{array}$

\subsubsection{Seahorse XFe 24 Analysis}

Medium and reagents used to assess MitoStress Stress, Glycolysis Stress and Fatty Acid Oxidation (FAO) using the $\mathrm{XF}^{\mathrm{e}} 24$ seahorse instrument are provided in the following tables.

Table 2.24: Seahorse Assay Medium for MitoStress Test Kit

Seahorse Assay Medium

Sodium Pyruvate

Glucose

Glutamine
Concentration/Amount/Volume

$1 \mathrm{mM}$

$25 \mathrm{mM}$

$2 \mathrm{mM}$

Top up with Seahorse XF Base Medium

(See Table 2.34 for details)

to required volume 
Table 2.25: Seahorse Fatty Acid Oxidation (FAO) Assay Substrate-Limited

\section{Medium}

Seahorse FAO Substrate-Limited Med.

Concentration/Amount/Volume

Glucose
Glutamine
Carnitine
FBS

$0.5 \mathrm{mM}$

$1 \mathrm{mM}$

FBS

$0.5 \mathrm{mM}$

$1 \%$

Top up with Seahorse XF Base Medium

(See Table 2.34 for details)

to required volume

Table 2.26: Seahorse FAO Assay Medium (KHB Medium)

$\begin{array}{cc}\text { Seahorse FAO Substrate-Limited Med. } & \text { Concentration/Amou } \\ \mathrm{NaCl} & 111 \mathrm{mM} \\ \mathrm{KCl} & 4.7 \mathrm{mM} \\ \text { Calcium Chloride } & 1.25 \mathrm{mM} \\ \text { Magnesium Sulphate } & 2 \mathrm{mM} \\ \text { Sodium Phosphate Monobasic } & 1.2 \mathrm{mM} \\ \text { Glucose } & 2.5 \mathrm{mM} \\ \text { Carnitine } & 0.5 \mathrm{mM} \\ \text { HEPES } & 5 \mathrm{mM}\end{array}$

Top up with MQ water to

required volume

Table 2.27: Seahorse FAO Assay Palmitate-BSA Solution

Seahorse FAO Substrate-Limited Med. Concentration/Amount/Volume

Sodium Palmitate $1 \mathrm{mM}$

BSA (Fatty acid free) $\quad 0.17 \mathrm{mM}$

$\mathrm{NaCl} 150 \mathrm{mM}$

Top up with MQ water to

required volume

\subsubsection{SMARTPool Small interfering RNA (siRNA) Resuspension}

Details of the $5 \mathrm{X}$ concentrated siRNA buffer used to resuspend lyophilized siRNA stock vials are provided in the following table.

Table 2.28: siRNA Buffer (5X)

$\begin{array}{cc}\text { siRNA Buffer }(5 \mathrm{X}) & \text { Concentration/Amount/Volume } \\ \mathrm{KCl} & 300 \mathrm{mM} \\ \mathrm{HEPES}(\mathrm{pH}=7.5) & 30 \mathrm{mM} \\ \mathrm{MgCl}_{2} & 1 \mathrm{mM}\end{array}$

Top up with DEP-C treated water

to required volume 


\subsubsection{Antibodies}

Primary and secondary antibodies used in this thesis are listed below. Details of antibody companies, catalogue number, dilution, host species (species the antibody was raised in) and blocking solution are provided.

Table 2.29: Primary Antibodies

\begin{tabular}{|c|c|c|c|c|c|}
\hline $\begin{array}{c}\text { Antibody } \\
\text { Name }\end{array}$ & Company & $\begin{array}{l}\text { Catalogue } \\
\text { Number }\end{array}$ & Dilution & Host & $\begin{array}{l}\text { Blocking } \\
\text { Solution }\end{array}$ \\
\hline $\begin{array}{l}\text { p-AMPK } \alpha \\
(\text { Thr172) }\end{array}$ & Cell Signaling & 2535 & $1: 1000$ & rabbit & $5 \%$ milk \\
\hline AMPK $\alpha$ & Cell Signaling & 2532 & $1: 1000$ & rabbit & $5 \%$ milk \\
\hline $\begin{array}{l}\text { p-eIF2 } \alpha \\
(\text { Ser51) }\end{array}$ & Cell Signaling & 9721 & $1: 500$ & rabbit & $5 \%$ BSA \\
\hline $\mathrm{CHOP}$ & Cell Signaling & 5554 & $1: 200$ & rabbit & $5 \%$ BSA \\
\hline $\begin{array}{l}\text { p-mTOR } \\
\text { (Ser2448) }\end{array}$ & Cell Signaling & 2971 & $1: 1000$ & rabbit & $5 \% \mathrm{BSA}$ \\
\hline mTOR & Cell Signaling & 2972 & $1: 1000$ & rabbit & $5 \%$ milk \\
\hline $\begin{array}{c}\text { p-FOXO3a } \\
(\text { Ser253) }\end{array}$ & Cell Signaling & 9466 & $1: 1000$ & rabbit & $5 \%$ BSA \\
\hline $\mathrm{FOXO3a}$ & Cell Signaling & 7497 & $1: 500$ & rabbit & $5 \%$ milk \\
\hline $\begin{array}{c}\text { p-FOXO1 } \\
\text { (Ser256) }\end{array}$ & Cell Signaling & 9461 & $1: 1000$ & rabbit & $5 \%$ milk \\
\hline FOXO1 & Cell Signaling & 2880 & 1:1000 & rabbit & $5 \%$ milk \\
\hline DRP1 & Cell Signaling & 8570 & $1: 1000$ & rabbit & $5 \%$ milk \\
\hline PINK1 & Abcam & ab23707 & $1: 1000$ & rabbit & $5 \%$ milk \\
\hline PARKIN & Abcam & ab130867 & 1:1000 & mouse & $5 \%$ milk \\
\hline COXIV & Abcam & ab14744 & $1: 500$ & mouse & PVP \\
\hline OXPHOS & Abcam & ab110413 & $1: 1000$ & mouse & $5 \%$ milk \\
\hline MFN1 & Abcam & ab57602 & $1: 1000$ & mouse & $5 \%$ milk \\
\hline PARIS & Abcam & 130867 & $1: 1000$ & rabbit & $5 \%$ milk \\
\hline HSP60 & Abcam & ab46798 & $1: 10000$ & rabbit & $5 \%$ milk \\
\hline MURF1 & Regeneron & (Gift) & $1: 500$ & mouse & $5 \%$ milk \\
\hline MFN2 & Santa Cruz & sc- 100560 & $1: 1000$ & mouse & $5 \%$ milk \\
\hline $\begin{array}{c}\mathrm{p}-\mathrm{AKT} 1 / 2 / 3 \\
(\text { Ser 473) }\end{array}$ & Santa Cruz & sc-7985 & $1: 1000$ & rabbit & $5 \%$ milk \\
\hline AKT1/2/3 & Santa Cruz & sc8312 & $1: 1000$ & rabbit & $5 \%$ milk \\
\hline PERK & Cell Signaling & sc-13073 & $1: 1000$ & rabbit & $5 \%$ milk \\
\hline ATROGIN-1 & Cell Signaling & PAB15627 & $1: 500$ & goat & $5 \%$ milk \\
\hline LC3 & Novus Bio. & NB100-2220 & $1: 500$ & rabbit & $5 \%$ milk \\
\hline PGC- $1 \alpha$ & Millipore & ab3242 & $1: 1000$ & rabbit & $5 \%$ milk \\
\hline FIS1 & BioVision & 3491R & $1: 1000$ & rabbit & $5 \%$ milk \\
\hline МyHC & DSHB & MF20 & $1: 10000$ & mouse & $5 \%$ milk \\
\hline
\end{tabular}




\begin{tabular}{|c|c|c|c|c|c|} 
MyLC & DSHB & T14C & $1: 10000$ & mouse & $5 \%$ milk \\
\hline GAPDH & Santa Cruz & sc-32233 & $1: 10000$ & mouse & $5 \%$ milk \\
\hline$\alpha$-TUBULIN & Sigma & T9026 & $1: 10000$ & mouse & $5 \%$ milk \\
\hline
\end{tabular}

Table 2.30: Secondary Antibodies

\begin{tabular}{|c|c|c|c|c|c|}
\hline $\begin{array}{c}\text { Antibody } \\
\text { Name }\end{array}$ & Company & $\begin{array}{c}\text { Catalogue } \\
\text { Number }\end{array}$ & Dilution & Host & $\begin{array}{c}\text { Blocking } \\
\text { Solution }\end{array}$ \\
\hline $\begin{array}{c}\text { Anti-mouse } \\
\text { HRP }\end{array}$ & Bio-Rad & 1706516 & $1: 5000$ & goat & $\begin{array}{c}\text { same as } \\
\text { primary }\end{array}$ \\
\hline $\begin{array}{c}\text { Anti-rabbit } \\
\text { HRP }\end{array}$ & Bio-Rad & 1706515 & $1: 5000$ & goat & $\begin{array}{c}\text { same as } \\
\text { primary }\end{array}$ \\
\hline $\begin{array}{c}\text { Anti-goat } \\
\text { HRP }\end{array}$ & Bio-Rad & 1721034 & $1: 2000$ & rabbit & $\begin{array}{c}\text { same as } \\
\text { primary }\end{array}$ \\
\hline
\end{tabular}

\subsubsection{Oligonucleotides}

Oligonucleotides were designed using NCBI primer designing tool (Primer-BLAST, NCBI, https://www.ncbi.nlm.nih.gov/tools/primer-blast/) and were purchased from Integrated DNA Technologies (IDT, Singapore). Pre-designed gene-specific SMARTpool siRNA, which target four different regions of the gene of interest and amplify a 150-200 base pairs region, were purchased from Dharmacon RNAi Technologies (Lafayette, CO, USA). Sequences of oligonucleotides and SMARTpool siRNA are provided in the tables below.

Table 2.31: Oligonucleotide Sequences

\begin{tabular}{|c|c|c|c|}
\hline $\begin{array}{l}\text { Gene } \\
\text { Symbol }\end{array}$ & $\begin{array}{c}\text { Accession } \\
\text { No/Reference }\end{array}$ & $\begin{array}{c}\text { Forward Primer Sequence (5'- } \\
\left.3^{\prime}\right)\end{array}$ & $\begin{array}{c}\text { Reverse Primer Sequence } \\
\left(5^{\prime}-3^{\prime}\right)\end{array}$ \\
\hline Mfnl & 261 & ATTGGGGAGGTGCTGTCTC & $\begin{array}{c}\text { TTCGGTCATAAGGTAGG } \\
\text { CTTT }\end{array}$ \\
\hline Mfn2 & 261 & AGATGTCCCTGCTCTTTTCTC & $\begin{array}{c}\text { TGTGTTCCTGTGGGTGTC } \\
\text { TT }\end{array}$ \\
\hline Opal & 261 & $\begin{array}{c}\text { GATGACACGCTCTCCAGTGA } \\
\text { AG }\end{array}$ & $\begin{array}{c}\text { CTCGGGGCTAACAGTAC } \\
\text { AACC }\end{array}$ \\
\hline Drpl & 261 & CGGTTCCCTAAACTTCACGA & $\begin{array}{c}\text { GCACCATTTCATTTGTCA } \\
\text { CG } \\
\end{array}$ \\
\hline Fisl & 261 & AAGTATGTGCGAGGGCTGTT & $\begin{array}{c}\text { GGCAGAGAGCAGGTGA } \\
\text { GG }\end{array}$ \\
\hline Mff & 261 & TCACATTTGGTGAGTGGGGC & $\begin{array}{c}\text { TTTTCCGGGACCCTCATT } \\
\text { CG }\end{array}$ \\
\hline$N d 1$ & 262 & TGCACCTACCCTATCACTC & $\begin{array}{c}\text { ATTGTTTGGGCTACGGC } \\
\text { TC }\end{array}$ \\
\hline$N d 2$ & 262 & ATGAGTAGGCCTGGAATT & $\begin{array}{c}\text { ATCAGAAGTGGAATGGG } \\
\text { G }\end{array}$ \\
\hline$N d 4$ & 262 & $\begin{array}{c}\text { ATAATTATAACTAGCTCAAT } \\
\text { CTGC }\end{array}$ & $\begin{array}{c}\text { TCGTAGTTGGAGTTTGC } \\
\text { TAG }\end{array}$ \\
\hline$N d 4 L$ & 262 & CTCACCATAGCCTTCTCAC & $\begin{array}{c}\text { CGTAATCTGTTCCGTAC } \\
\text { GTG }\end{array}$ \\
\hline
\end{tabular}




\begin{tabular}{|c|c|c|c|}
\hline$N d 6$ & 262 & $\begin{array}{c}\text { TGTATGAGGTTGATGATGTT } \\
\text { GG }\end{array}$ & $\begin{array}{c}\text { CCGCAAACAAAGATCAC } \\
\text { CC }\end{array}$ \\
\hline$C y t b$ & 262 & $\begin{array}{c}\text { CTTTGGGTCCCTTCTAGGAG } \\
\text { TCTG } \\
\end{array}$ & $\begin{array}{c}\text { GCTGTGGCTATGACTGC } \\
\text { GAACAG }\end{array}$ \\
\hline CoI & 262 & GCTGAAGGAGAAGGAGAA & $\begin{array}{c}\text { ATACACATAGCTCTTCT } \\
\text { CCC }\end{array}$ \\
\hline Atp6 & 262 & $\begin{array}{c}\text { CCACACACCAAAAGGACGA } \\
\text { ACATGA }\end{array}$ & $\begin{array}{c}\text { CGGACTGCTAATGCCAT } \\
\text { TGGTTG } \\
\end{array}$ \\
\hline Ndufa9 & 262 & CATTACTGCAGAGCCACT & $\begin{array}{c}\text { ATCAGACGAAGGTGCAT } \\
\text { GAT }\end{array}$ \\
\hline Ndufb6 & 262 & ATAACTTTTTGCGGGACGGG & $\begin{array}{c}\text { CAGGAAAATCTCTCATT } \\
\text { GGTG }\end{array}$ \\
\hline Ndufs 3 & 262 & AGGAACATGGCGGCGGCTGC & $\begin{array}{c}\text { ATTTCAGCCACATACTC } \\
\text { TCC } \\
\end{array}$ \\
\hline Sdha & 262 & $\begin{array}{c}\text { CATACTGTTGCAGCAGCACA } \\
\text { GG }\end{array}$ & $\begin{array}{c}\text { CCACCAAATGCACGCTG } \\
\text { ATA }\end{array}$ \\
\hline Uqcrc1 & 262 & CCTACAGCACTCGAGAGCAC & $\begin{array}{c}\text { AGGTGTGCCCTGGAATG } \\
\text { CTG }\end{array}$ \\
\hline Uqcrc2 & 262 & TCCCTCAAAGTTGCCCC & $\begin{array}{c}\text { GCAAGACGTAGTAAATG } \\
\text { TGAG }\end{array}$ \\
\hline Uqcrfs & 262 & GATGTCAAGGTGCCCGACTT & $\begin{array}{c}\text { GATCTCGATCTTCGACA } \\
\text { TGG } \\
\end{array}$ \\
\hline $\operatorname{Cox} 4$ & 262 & GAGCACATGGGAGTGTTGTG & $\begin{array}{c}\text { CTGTCTTCCATTCATTGG } \\
\text { TGC } \\
\end{array}$ \\
\hline Atp5 & 262 & GGTCATCCTTTGTTGGTGC & $\begin{array}{c}\text { GAGAATTCCACCATCCC } \\
\text { TTC } \\
\end{array}$ \\
\hline Parkin & $\begin{array}{c}\text { XM_006523 } \\
336.3\end{array}$ & AAACCGGATGAGTGGTGAGT & $\begin{array}{c}\text { AGCTACCGACGTGTCCT } \\
\text { TGT }\end{array}$ \\
\hline Pinkl & NM_026880.2 & GCTGATCGAGGAGAAGCAG & $\begin{array}{c}\text { GATAATCCTCCAGACGG } \\
\text { AAGC }\end{array}$ \\
\hline Nedd4 & $\begin{array}{c}\text { NM_010890 } \\
.3\end{array}$ & CTCTCGGAGGACGAGGTATG & $\begin{array}{c}\text { CTCTCGGAGGACGAGGT } \\
\text { ATG } \\
\end{array}$ \\
\hline FoxO3 & NM_019740.2 & $\begin{array}{c}\text { CCTACTTCAAGGATAAGGGC } \\
\text { GAC }\end{array}$ & $\begin{array}{c}\text { GGAGCTCCAGGTACGGA } \\
\text { TCA }\end{array}$ \\
\hline$M u R F 1$ & $\begin{array}{c}\text { NM_00103904 } \\
8.2\end{array}$ & $\begin{array}{c}\text { ACGAGAAGAAGAGCGAGCT } \\
\text { G }\end{array}$ & $\begin{array}{c}\text { GCCTTCATTCTGAACGC } \\
\text { GCATG }\end{array}$ \\
\hline Atrogin-1 & NM_026346.3 & GCGTGTTCTCTGGCAACATA & $\begin{array}{c}\text { CTTGGCACTTGAGAGGA } \\
\text { AGG }\end{array}$ \\
\hline Trim72 & $\begin{array}{c}\text { NM_00107993 } \\
2.3\end{array}$ & $\begin{array}{c}\text { GGAGTGCTCAGACCAGAAG } \\
\text { G }\end{array}$ & $\begin{array}{c}\text { GAGAAGAGGTGCAGGG } \\
\text { ACTG }\end{array}$ \\
\hline $\begin{array}{c}\text { mtDNA } \\
(\mathrm{CoI})\end{array}$ & 263 & GCCCCAGATATAGCATTCCC & $\begin{array}{c}\text { GTTCATCCTGTTCCTGCT } \\
\text { CC } \\
\end{array}$ \\
\hline $\begin{array}{c}\text { nuDNA } \\
(18 S)\end{array}$ & 263 & TAGAGGGACAAGTGGCGTTC & $\begin{array}{c}\text { CGCTGAGCCAGTCAGTG } \\
\mathrm{T}\end{array}$ \\
\hline Ube $2 c$ & NM_026785.2 & GCCGGCACCGTATATGAAGA & $\begin{array}{c}\text { GTGGGTGCGTTGTAAGG } \\
\text { GTA }\end{array}$ \\
\hline Fbxl22 & NM_175206.4 & ACCCTTTCTGGGCATTCACG & $\begin{array}{c}\text { CACAGCAGACACTCCCG } \\
\text { ATT }\end{array}$ \\
\hline Rnfl44a & NM_080563.4 & CGGGACCAGGTACTTTGTGT & $\begin{array}{c}\text { GCACTAGGGGTCCATCC } \\
\text { AAC }\end{array}$ \\
\hline Traip & NM_011634.3 & ACTAGCAAGAATGGCAGCCC & $\begin{array}{c}\text { AGTCCTGAACCCAAGCC } \\
\text { CTA }\end{array}$ \\
\hline Trim $43 b$ & $\begin{array}{c}\text { NM_00117088 } \\
4.1\end{array}$ & CAAGTGTGTGACCCACAAGG & $\begin{array}{c}\text { ACCACCACAAGGTCGTT } \\
\text { CTC }\end{array}$ \\
\hline Trim59 & NM_025863.3 & AATTGATGAAGTCCGCCAAC & $\begin{array}{c}\text { CAGGAACATGGCGTTCT } \\
\text { TTT }\end{array}$ \\
\hline
\end{tabular}




\begin{tabular}{|c|c|c|c|}
\hline Zfp598 & NM_183149.1 & $\begin{array}{c}\text { GGTGAGGACTACGAGGAGG } \\
\text { T }\end{array}$ & $\begin{array}{c}\text { CTTGTACCTCCAGCTTCC } \\
\text { CC }\end{array}$ \\
\hline Usp18 & NM_011909.2 & CAGGAGTCCCTGATTTGCGT & $\begin{array}{c}\text { GGGCTGGACGAAACATC } \\
\text { TCA }\end{array}$ \\
\hline Bip & $\begin{array}{c}\text { NM_001163 } \\
434.1\end{array}$ & TGTGTGTGAGACCAGAACCG & $\begin{array}{c}\text { TGCCCACATCCTCCTTCT } \\
\text { TG }\end{array}$ \\
\hline Chop & $\begin{array}{c}\text { NM_00129018 } \\
3.1\end{array}$ & $\begin{array}{c}\text { CCTGAGGAGAGAGAACCTG } \\
\text { GTC }\end{array}$ & $\begin{array}{c}\text { CTTCGTTTCCTGGGGAT } \\
\text { GAGAT }\end{array}$ \\
\hline Ire1 $\alpha$ & M_023913.2 & TGCTGTTAGCAAGAGGACGG & $\begin{array}{c}\text { GGCCTGAACCAATTCTG } \\
\text { GGA }\end{array}$ \\
\hline Gapdh & NM_008084 & $\begin{array}{c}\text { GATGATGACCCGTTTGGCTC } \\
\text { C }\end{array}$ & $\begin{array}{c}\text { ACGCTCGTGGAAAGAAA } \\
\text { AGA }\end{array}$ \\
\hline Ppia & NM_008907.1 & $\begin{array}{c}\text { GCATACGGGTCCTGGCATCT } \\
\text { TGTCC }\end{array}$ & $\begin{array}{c}\text { GTGGCAAAGTGAGATTG } \\
\text { TTGCC }\end{array}$ \\
\hline
\end{tabular}

Table 2.32: SMARTpool siRNA Sequences

\begin{tabular}{|c|c|c|c|}
\hline siRNA & $\begin{array}{l}\text { Catalogue } \\
\text { Number }\end{array}$ & \multicolumn{2}{|c|}{ Sequence $\left(5^{\prime}-3^{\prime}\right)$} \\
\hline \multirow{2}{*}{$\begin{array}{l}\text { Non-silencing } \\
\text { control }\end{array}$} & \multirow{2}{*}{$\begin{array}{l}\text { D-001206- } \\
13\end{array}$} & $\begin{array}{c}\text { UAGCGACUAAACACAUC } \\
\text { AA }\end{array}$ & $\begin{array}{c}\text { UAAGGCUAUGAAGAGAU } \\
\text { AC }\end{array}$ \\
\hline & & $\begin{array}{c}\text { AUGUAUUGGCCUGUAUU } \\
\text { AG }\end{array}$ & $\begin{array}{c}\text { AUGAACGUGAAUUGCUC } \\
\text { AA }\end{array}$ \\
\hline \multirow{2}{*}{$\begin{array}{l}\text { Murine Parkin- } \\
\text { specific }\end{array}$} & \multirow{2}{*}{$\begin{array}{l}\text { M-065413- } \\
01\end{array}$} & $\begin{array}{c}\text { CGAAUCACCUGACGGUU } \\
\text { CA }\end{array}$ & $\begin{array}{c}\text { ACACGUCGGUAGCUUUG } \\
\text { AA }\end{array}$ \\
\hline & & $\begin{array}{c}\text { ACUCAACGAUCGGCAGU } \\
\text { UU }\end{array}$ & $\begin{array}{c}\text { GAGGAAUGCGUGCUGCA } \\
\text { AA }\end{array}$ \\
\hline
\end{tabular}

\subsubsection{Chemical, Reagent and Kit Inventory List}

Details of chemicals, reagents and kits used in this study are listed in following tables.

Table 2.33: List of Chemicals Used in This Thesis

\begin{tabular}{|c|c|c|}
\hline Chemical & Company & $\begin{array}{l}\text { Catalogue } \\
\text { Number }\end{array}$ \\
\hline 2-propanol (Isopropanol) & Merck KGaA, Darmstadt, Germany & 109634 \\
\hline Acetic acid & Merck KGaA, Darmstadt, Germany & 100056 \\
\hline Ammonium persulfate & Sigma-Aldrich, St. Louis, MO, USA & A 3678 \\
\hline$\beta$-mercaptoethanol & Sigma-Aldrich, St. Louis, MO, USA & M6250 \\
\hline $\begin{array}{l}\text { Bovine serum albumin } \\
\text { (BSA) }\end{array}$ & Sigma-Aldrich, St. Louis, MO, USA & A3675 \\
\hline Bromophenol blue & Sigma-Aldrich, St. Louis, MO, USA & 114391 \\
\hline Calcium chloride $\left(\mathrm{CaCl}_{2}\right)$ & Sigma-Aldrich, St. Louis, MO, USA & 449709 \\
\hline $\begin{array}{c}\text { Carbonyl cyanide 3- } \\
\text { chlorophenylhydrazone } \\
\text { (CCCP) }\end{array}$ & Sigma-Aldrich, St. Louis, MO, USA & $\mathrm{C} 2759$ \\
\hline Chloroform & Merck KGaA, Darmstadt, Germany & 102445 \\
\hline Chloroquine & Sigma-Aldrich, St. Louis, MO, USA & C6628 \\
\hline
\end{tabular}




\begin{tabular}{|c|c|c|}
\hline Collagenase & Sigma-Aldrich, St. Louis, MO, USA & $\mathrm{C} 0130$ \\
\hline D-glucose & Sigma-Aldrich, St. Louis, MO, USA & G7021 \\
\hline $\begin{array}{l}\text { Dimethyl sulfoxide } \\
\text { (DMSO) }\end{array}$ & Sigma-Aldrich, St. Louis, MO, USA & 276855 \\
\hline Eosin-Y solution & Merck KGaA, Darmstadt, Germany & 109844 \\
\hline Ethanol & Merck KGaA, Darmstadt, Germany & 107017 \\
\hline Ethidium bromide & Bio-Rad, Hercules, CA, USA & 1610433 \\
\hline $\begin{array}{l}\text { Ethylenediaminetetraacetic } \\
\text { Acid (EDTA) }\end{array}$ & Sigma-Aldrich, St. Louis, MO, USA & E9884 \\
\hline Fatty acid free-BSA & Sigma-Aldrich, St. Louis, MO, USA & 3117057001 \\
\hline Formaldehyde & Sigma-Aldrich, St. Louis, MO, USA & 252549 \\
\hline Formamide & Sigma-Aldrich, St. Louis, MO, USA & F9037 \\
\hline L-Glutamine & $\begin{array}{l}\text { Thermo Fisher Scientific, Waltham, } \\
\text { MA, USA }\end{array}$ & $21051-024$ \\
\hline Glycerol & Sigma-Aldrich, St. Louis, MO, USA & 536407 \\
\hline Glycine & Bio-Rad, Hercules, CA, USA & 1610718 \\
\hline Hematoxylin, Gill III & Merck KGaA, Darmstadt, Germany & 105174 \\
\hline HEPES & Sigma-Aldrich, St. Louis, MO, USA & H7006 \\
\hline L-Carnitine hydrochloride & Sigma-Aldrich, St. Louis, MO, USA & $\mathrm{C} 0283$ \\
\hline $\begin{array}{c}\text { Magnesium chloride } \\
\left(\mathrm{MgCl}_{2}\right)\end{array}$ & Sigma-Aldrich, St. Louis, MO, USA & M8266 \\
\hline Magnesium sulphate & Sigma-Aldrich, St. Louis, MO, USA & M5921 \\
\hline Methanol & Merck KGaA, Darmstadt, Germany & 106009 \\
\hline MOPS & Sigma-Aldrich, St. Louis, MO, USA & M1254 \\
\hline PMSF & Sigma-Aldrich, St. Louis, MO, USA & P7626 \\
\hline Polyethylene Glycol (PEG) & Sigma-Aldrich, St. Louis, MO, USA & 202444 \\
\hline Polyvinylpyrrolidone (PVP) & Sigma-Aldrich, St. Louis, MO, USA & 234257 \\
\hline Potassium chloride $(\mathrm{KCl})$ & Sigma-Aldrich, St. Louis, MO, USA & P9541 \\
\hline Sodium acetate & Sigma-Aldrich, St. Louis, MO, USA & S2889 \\
\hline Sodium bicarbonate & Sigma-Aldrich, St. Louis, MO, USA & S5761 \\
\hline Sodium chloride $(\mathrm{NaCl})$ & 1st Base, Singapore & BIO-1110 \\
\hline Sodium deoxycholate & Sigma-Aldrich, St. Louis, MO, USA & D6750 \\
\hline $\begin{array}{l}\text { Sodium dodecyl sulfate } \\
\text { (SDS) }\end{array}$ & Sigma-Aldrich, St. Louis, MO, USA & L3771 \\
\hline Sodium floride $(\mathrm{NaF})$ & Sigma-Aldrich, St. Louis, MO, USA & S7920 \\
\hline Sodium hydroxide $(\mathrm{NaOH})$ & Sigma-Aldrich, St. Louis, MO, USA & S0899 \\
\hline $\begin{array}{c}\text { Sodium orthovanadate } \\
\left(\mathrm{Na}_{3} \mathrm{VO}_{4}\right)\end{array}$ & Sigma-Aldrich, St. Louis, MO, USA & S6508 \\
\hline Sodium palmitate & Sigma-Aldrich, St. Louis, MO, USA & P9767 \\
\hline $\begin{array}{l}\text { Sodium phosphate } \\
\text { monobasic }\end{array}$ & Sigma-Aldrich, St. Louis, MO, USA & S0751 \\
\hline
\end{tabular}




\begin{tabular}{|c|c|c|}
\hline $\begin{array}{c}\text { Tetramethylethylenediamine } \\
\text { (TEMED) }\end{array}$ & Bio-Rad, Hercules, CA, USA & 1610801 \\
\hline Tris base & 1st Base, Singapore & BIO-1400 \\
\hline Tween-20 & $\begin{array}{c}\text { Fisher Scientific, Waltham, MA, } \\
\text { United States }\end{array}$ & PRH5151 \\
\hline Xylene & Sigma-Aldrich, St. Louis, MO, USA & 214736 \\
\hline
\end{tabular}

Table 2.34: List of Reagents Used in This Thesis

\begin{tabular}{|c|c|c|}
\hline Reagent & Company & $\begin{array}{l}\text { Catalogue } \\
\text { Number }\end{array}$ \\
\hline $1 \mathrm{~kb}$ plus DNA ladder & $\begin{array}{c}\text { Thermo Fisher Scientific, Waltham, } \\
\text { MA, USA }\end{array}$ & 10787018 \\
\hline $\begin{array}{l}30 \% \text { acrylamide/bis } \\
\text { solution }\end{array}$ & Bio-Rad, Hercules, CA, USA & 1610156 \\
\hline $\begin{array}{l}\text { BlueJuice }^{\mathrm{TM}} \text { gel loading } \\
\text { buffer }\end{array}$ & $\begin{array}{c}\text { Thermo Fisher Scientific, Waltham, } \\
\text { MA, USA }\end{array}$ & 10816015 \\
\hline $\begin{array}{l}\text { Chick embryo extract } \\
\text { (CEE) }\end{array}$ & $\begin{array}{c}\text { United States Biological, Salem, } \\
\text { MA, USA }\end{array}$ & C3999 \\
\hline $\begin{array}{l}\text { cOmplete }{ }^{\mathrm{TM}} \text { Mini EDTA- } \\
\text { free Protease Inhibitor }\end{array}$ & $\begin{array}{c}\text { Sigma-Aldrich, St. Louis, MO, } \\
\text { USA }\end{array}$ & 4693159001 \\
\hline DharmaFECT $^{\circledR} 1$ & $\begin{array}{c}\text { Dharmacon RNAi Technologies, } \\
\text { Lafayette, CO, USA }\end{array}$ & $\mathrm{T}-2001$ \\
\hline DPX & $\begin{array}{c}\text { Sigma-Aldrich, St. Louis, MO, } \\
\text { USA }\end{array}$ & 44581 \\
\hline Fetal bovine serum (FBS) & Gibco, Carlsbad, CA, USA & 10082147 \\
\hline $\begin{array}{c}\text { High Glucose Dulbecco's } \\
\text { Modified Eagle Medium } \\
\text { (DMEM) }\end{array}$ & Gibco, Carlsbad, CA, USA & 10566016 \\
\hline Horse serum (HS) & Gibco, Carlsbad, CA, USA & 16050122 \\
\hline IGEPAL (NP-40) & $\begin{array}{c}\text { Sigma-Aldrich, St. Louis, MO, } \\
\text { USA }\end{array}$ & I8896 \\
\hline LDS sample buffer & $\begin{array}{c}\text { Thermo Fisher Scientific, Waltham, } \\
\text { MA, USA }\end{array}$ & NP0008 \\
\hline Matrigel $^{\circledR}$ & Corning Inc, corning, NY, USA & 354248 \\
\hline MitoTracker ${ }^{\circledR}$ Green FM & $\begin{array}{c}\text { Thermo Fisher Scientific, Waltham, } \\
\text { MA, USA }\end{array}$ & M7514 \\
\hline $\begin{array}{l}\text { MitoTracker }{ }^{\circledR} \text { Red } \\
\text { CMXRos }\end{array}$ & $\begin{array}{c}\text { Thermo Fisher Scientific, Waltham, } \\
\text { MA, USA }\end{array}$ & M7512 \\
\hline $\begin{array}{l}\text { Novex }{ }^{\mathrm{TM}} \text { Tris Glycine SDS } \\
\text { Running Buffer }\end{array}$ & $\begin{array}{c}\text { Thermo Fisher Scientific, Waltham, } \\
\text { MA, USA }\end{array}$ & LC2675 \\
\hline $\begin{array}{c}\text { NuPAGE }^{\circledR} 4-12 \% \text { bis-tris } \\
\text { precast gel }\end{array}$ & Invitrogen, Carlsbad, CA, USA & NP0321BOX \\
\hline $\begin{array}{c}\text { NuPAGE }^{\circledR} \text { MES Running } \\
\text { Buffer } \\
\end{array}$ & $\begin{array}{c}\text { Thermo Fisher Scientific, Waltham, } \\
\text { MA, USA }\end{array}$ & NP0002 \\
\hline OCT & VWR, Radnor, PA. USA & $25608-930$ \\
\hline Opti-MEM ${ }^{\mathrm{TM}}$ & Gibco, Carlsbad, CA, USA & 31985070 \\
\hline
\end{tabular}




\begin{tabular}{|c|c|c|}
\hline $\begin{array}{l}\text { Oxoid }^{\mathrm{TM}} \text { Phosphate- } \\
\text { Buffered Saline Tablet }\end{array}$ & $\begin{array}{c}\text { Thermo Fisher Scientific, Waltham, } \\
\text { MA, USA }\end{array}$ & BR0014G \\
\hline Penicillin/Streptomycin & Gibco, Carlsbad, CA, USA & 15140122 \\
\hline PhosSTOPтм & $\begin{array}{c}\text { Sigma-Aldrich, St. Louis, MO, } \\
\text { USA }\end{array}$ & PHOSS-RO \\
\hline Ponceau S & $\begin{array}{c}\text { Sigma-Aldrich, St. Louis, MO, } \\
\text { USA }\end{array}$ & P7170 \\
\hline RPMI, no methionine & $\begin{array}{c}\text { Thermo Fisher Scientific, Waltham, } \\
\text { MA, USA }\end{array}$ & A1451701 \\
\hline $\begin{array}{c}\text { Seahorse XF Assay } \\
\text { Medium }\end{array}$ & $\begin{array}{c}\text { Agilent Technologies, Santa Clara, } \\
\text { CA, USA }\end{array}$ & $102365-100$ \\
\hline Seahorse XF Base Medium & $\begin{array}{c}\text { Agilent Technologies, Santa Clara, } \\
\text { CA, USA }\end{array}$ & $102353-100$ \\
\hline $\begin{array}{c}\text { Seahorse XF Calibrant } \\
\text { solution }\end{array}$ & $\begin{array}{c}\text { Agilent Technologies, Santa Clara, } \\
\text { CA, USA }\end{array}$ & $100840-000$ \\
\hline $\begin{array}{l}\text { See Blue Plus2 protein } \\
\text { standard }\end{array}$ & $\begin{array}{c}\text { Thermo Fisher Scientific, Waltham, } \\
\text { MA, USA }\end{array}$ & LC5925 \\
\hline $\begin{array}{c}\text { SsoFast }^{\mathrm{TM}} \text { EvaGreen } \\
\text { Supermix }\end{array}$ & Bio-Rad, Hercules, CA, USA & $172-5200$ \\
\hline $\begin{array}{l}\text { Tris-Glycine lds sample } \\
\text { buffer }\end{array}$ & $\begin{array}{c}\text { Thermo Fisher Scientific, Waltham, } \\
\text { MA, USA }\end{array}$ & LC2676 \\
\hline TRIzol $^{\circledR}$ & $\begin{array}{c}\text { Thermo Fisher Scientific, Waltham, } \\
\text { MA, USA }\end{array}$ & 15596026 \\
\hline UltraPure $^{\mathrm{TM}}$ agarose & $\begin{array}{c}\text { Thermo Fisher Scientific, Waltham, } \\
\text { MA, USA }\end{array}$ & $16500-500$ \\
\hline $\begin{array}{l}\text { Western Lightning }{ }^{\circledR} \text { Plus- } \\
\text { ECL }\end{array}$ & Perkin Elmer, Waltham, MA, USA & NEL105001EA \\
\hline
\end{tabular}

Table 2.35: List of Kits Used in This Thesis

\begin{tabular}{|c|c|c|}
\hline Kit Name & Company & $\begin{array}{c}\text { Catalogue } \\
\text { Number }\end{array}$ \\
\hline $\begin{array}{c}\text { Agilent RNA 6000 Nano } \\
\text { Kit }\end{array}$ & $\begin{array}{c}\text { Agilent Technologies, Santa Clara, } \\
\text { CA, USA }\end{array}$ & $5067-1511$ \\
\hline $\begin{array}{c}\text { Click-iT }{ }^{\circledR} \text { HPG Alexa } \\
\text { Fluor }{ }^{\circledR} \text { Protein Synthesis } \\
\text { Assay Kit }\end{array}$ & $\begin{array}{c}\text { Thermo Fisher Scientific, Waltham, } \\
\text { MA, USA }\end{array}$ & C10428 \\
\hline iScript $^{\mathrm{TM}}$ cDNA Kit & Bio-Rad, Hercules, CA, USA & $170-8890$ \\
\hline $\begin{array}{c}\text { mirVana } \\
\text { Ism miRNA }\end{array}$ & $\begin{array}{c}\text { Thermo Fisher Scientific, Waltham, Kit } \\
\text { MA, USA }\end{array}$ & AM1560 \\
\hline $\begin{array}{c}\text { Mitochondria Isolation Kit } \\
\text { for Cultured Cells }\end{array}$ & $\begin{array}{c}\text { Thermo Fisher Scientific, Waltham, USA } \\
\text { MAteasome-Glo }\end{array}$ & 89874 \\
\hline $\begin{array}{c}\text { Substrate Cell-Based Assay } \\
\text { System }\end{array}$ & Promega, Madison, WI, USA & G1180 \\
\hline Q1Aamp ${ }^{\circledR}$ DNA Mini Kit & Qiagen, Valencia, CA, USA & 51304 \\
\hline $\begin{array}{c}\text { Seahorse XF Cell Mito } \\
\text { Stress Test Kit }\end{array}$ & $\begin{array}{c}\text { Agilent Technologies, Santa Clara, } \\
\text { CA, USA }\end{array}$ & $103020-100$ \\
\hline
\end{tabular}




\subsection{Methods}

\subsubsection{Animal Studies}

Park2 ${ }^{\mathrm{tm} 1 \mathrm{Shn}}$ mice in C57BL/6 background were obtained from The Jackson Laboratory (Sacramento, CA, USA). Wild type C57BL/6 mice were obtained from Biological Resource Centre (BRC, Singapore). Mice were housed in the Nanyang Technological University (NTU) Animal House Facility (Singapore), in temperature $\left(20-22^{\circ} \mathrm{C}\right)$ and light (12 hours light/dark) controlled rooms. All animal experiments were approved by the Institutional Animal Ethics Committee (IACUC, Singapore) and were conducted only after successful completion of the Responsible Care and Use of Laboratory Animal Course (RCULAC, Singapore).

\subsubsection{C2C12 Cell Culture and Treatments}

$\mathrm{C} 2 \mathrm{C} 12$ mouse myoblasts were obtained from ATCC (Manassas, VA, USA) and have been described previously ${ }^{264}$. For all studies, C2C12 myoblasts were grown at $37^{\circ} \mathrm{C}$ in a $5 \% \mathrm{CO}_{2}$ incubator. Myoblast proliferation was maintained with proliferation medium, which consists of high-glucose (4.5 g/L) Dulbecco's Modified Eagle Medium (DMEM; GE Healthcare, Piscataway, NJ, USA), supplemented with 10\% Fetal Bovine Serum (FBS) and 1\% Penicillin/Streptomycin (P/S; Gibco, Carlsbad, CA, USA). To induce differentiation of myoblasts into myotubes, myoblasts were seeded at the density of 25000 cells $/ \mathrm{cm}^{2}$ and grown in low serum differentiation medium, which consists of DMEM supplemented with 2\% Horse Serum (HS) and 1\% P/S (Gibco, Carlsbad, CA, USA), for $72 \mathrm{~h}$. Myoblasts fully differentiate into myotubes within $72 \mathrm{~h}$, as previously confirmed before ${ }^{265}$. For CCCP treatment, $72 \mathrm{~h}$ differentiated myotubes were treated with either $10 \mu \mathrm{M}$ CCCP or an equal volume of DMSO vehicle control (Sigma-Aldrich, St. Louis, MO, USA) for $24 \mathrm{~h}$. For chloroquine (CQ) treatment, $72 \mathrm{~h}$ differentiated myotubes were treated with either $100 \mu \mathrm{M}$ CQ or DMSO for an additional $6 \mathrm{~h}$ (SigmaAldrich, St. Louis, MO, USA).

\subsubsection{Transient Transfection of SMARTpool siRNA into C2C12 Myoblasts and Myotubes}

Myoblasts were seeded in 6-well plates, at a density of 15000 cells $/ \mathrm{cm}^{2}$ and maintained in $2 \mathrm{ml} /$ well of proliferation medium lacking antibiotics (without P/S). For 
myotube transfection, myoblasts were seeded as 20000 cells $/ \mathrm{cm}^{2}$ and differentiated for $72 \mathrm{~h}$ in differentiation medium. On the day of transfection, excess medium was removed to get a final volume of $1.6 \mathrm{ml} /$ well. Transfection medium was prepared as follows: 5 nmol lyophilized SMARTpool siRNAs were resuspended in $250 \mu 1$ of 5X siRNA Buffer to obtain a $20 \mu \mathrm{M}$ stock solution. siRNA solutions were further diluted with DEPC water to a concentration of $5 \mu \mathrm{M}$ ( $1^{\text {st }}$ Base, Singapore) just before transfection. A total of 10 $\mu$ diluted siRNA solution ( $25 \mathrm{nM}$ final) and $8 \mu$ DharmaFECT ${ }^{\circledR} 1$ Transfection Reagent per well were added into two different tubes, each containing $200 \mu \mathrm{l}$ Opti-MEMTM medium (Dharmacon RNAi Technologies, Lafayette, CO, USA). After incubation for 5 minutes at room temperature, each siRNA-Opti-MEM mixture was added into respective tubes containing DharmaFECT-Opti-MEM dropwise, while gently tapping the bottom of the micro centrifuge tubes. Following 20 minutes incubation at room temperature, the siRNA-liposome solution was added into respective wells dropwise while rocking the plate. Transfected cells were incubated for either $24 \mathrm{~h}$ or $48 \mathrm{~h}$ and were subjected to a medium change prior to sample collection. Use of antibiotics was avoided during transfections.

\subsubsection{Murine Primary Myoblast Isolation}

Four- to six-week-old wild type and Parkin KO male mice were euthanatized with $\mathrm{CO}_{2}$. Hind limb muscles were dissected and collected in PBS solution inside conical tubes, with each tube containing hind limb muscle tissue from no more than $2 \frac{1}{2}$ mice. Samples were transferred into gentleMACS ${ }^{\text {TM }}$ C-tubes and mechanical digestion was performed using the gentleMACS dissociator (Miltenyi Biotec, Singapore). Muscle samples were dissociated by running program " $m \_$muscle_O1" preinstalled by the manufacturer twice and separated from lipids (supernatant) by centrifuging at 3000xrpm for 5 minutes. Subsequently, samples were digested for 45 minutes in $0.2 \%$ collagenase solution (Sigma-Aldrich, St. Louis, MO, USA) in a $37^{\circ} \mathrm{C}$ shaking incubator at $120 \mathrm{xrpm}$. During digestion, samples were further dissociated every 15 minutes by running the m_muscle_01 program. Following digestion, the collagenase-muscle solution was topped up to $50 \mathrm{ml}$ by adding PBS and samples were centrifuged at $3000 \mathrm{xrpm}$ for 15 minutes. The supernatant was then discarded and the remaining pellet was resuspended in $10 \mathrm{ml}$ PBS. Pellets were triturated for 5 minutes using a $10 \mathrm{ml}$ serological pipette and subsequently applied to $100 \mu \mathrm{m}$ cell strainers placed on $50 \mathrm{ml}$ canonical tubes. The 
flow-through was then topped up to $50 \mathrm{ml}$ with PBS and centrifuged at 3000xrpm for 15 minutes. The resulting cell pellet was resuspended in $10 \mathrm{ml} / \mathrm{mouse}$ proliferation medium (high-glucose DMEM [Gibco, Carlsbad, CA, USA], 20\% Fetal Bovine Serum, 10\% Horse Serum, 1\% Penicillin/Streptomycin and 1\% Chick Embryo Extract [CEE] [United States Biological, Salem, MA, USA]). Populations of pure primary myoblasts were generated using a pre-plating technique. Upon initial resuspension of the cell pellet in proliferation medium, cells are plated onto uncoated $10 \mathrm{~cm}$ cell culture dishes for 3-4 h, which is referred to as pre-plate 1 (PP1). PP1 is the initial step in the isolation procedure that allows for the separation of fibroblasts from myoblasts, as fibroblasts adhere more rapidly to uncoated cell culture dishes, when compared to myoblast populations. Following PP1, cells are transferred to new $10 \mathrm{~cm}$ cell culture dishes coated with a $10 \%$ matrigel $^{\circledR}$ solution (Corning Inc, corning, NY, USA) dissolved in pre-chilled DMEM and are allow to attach for a further $24 \mathrm{~h}$, which is referred to as preplate 2 (PP2). The medium from PP2 plates is then transferred to a third matrigel ${ }^{\circledR}$ coated $10 \mathrm{~cm}$ cell culture dish and allowed to settle for a further $24 \mathrm{~h}$, which is termed pre-plate 3 (PP3). Cells adhered during PP2 and PP3 are myoblasts and both PP2 and PP3 populations of myoblasts were pooled for downstream studies. Primary myoblasts were maintained and differentiated on matrige ${ }^{\circledR}$ coated plates as described under Sections 2.2.2 and 2.2.4.

\subsubsection{Tissue Dissection}

4-week-old mice were euthanized through $\mathrm{CO}_{2}$ asphyxiation. M. quadriceps (Quad), M. gastrocnemius (Gas), M. tibialis anterior (TA), M. soleus (Sol) and $M$. extensor digitorum longus (EDL) hind limb skeletal muscle were dissected. Tissue weights, body weights and tibia lengths were recorded for each mouse. Tissue samples were snap frozen in liquid nitrogen for subsequent biochemical analyses or embedded in optimum cutting temperature (OCT) compound using liquid nitrogen cooled isopentane (Sigma-Aldrich, St. Louis, MO, USA) for subsequent histological analysis and myofiber cross-sectional area measurement.

\subsubsection{Protein Extraction and Western Blot Analysis}

To extract total cell protein, cells were lysed in complete RIPA buffer (1\% (v/v) IGEPAL, 0.1\% SDS, 0.5\% (w/v) Na Deoxycholate (w/v), $50 \mathrm{mM} \mathrm{NaF,} 0.2 \mathrm{mM}$ 
$\mathrm{Na}_{3} \mathrm{VO}_{4}, 1 \mathrm{mM}$ PMSF, cOmplete ${ }^{\mathrm{TM}}$ Mini EDTA-free Protease Inhibitor, PhosSTOP ${ }^{\mathrm{TM}}$ (Sigma-Aldrich, St. Louis, MO, USA)) after washing once with ice-cold PBS. Cells were kept on ice for 30 minutes and triturated 25 times using a 26 g needle assembled onto a $1 \mathrm{ml}$ syringe. To isolate proteins from tissues, approximately $30 \mathrm{mg}$ of each snap frozen tissue was cut on dry ice and homogenized using the Qiagen Tissue Lyser-II at a frequency of 30 seconds $^{-1}$, for $3 \times 2$ minutes (Qiagen, Valencia, CA, USA). Cell lysates and muscle homogenates were then centrifuged at $10000 \mathrm{xg}$ for 10 minutes at $4{ }^{\circ} \mathrm{C}$, with protein containing supernatants collected into fresh micro centrifuge tubes. Finally, each protein sample was quantified using Bio-Rad (Bradford) protein assay reagent, as per the manufacturer's instructions (Bio-Rad, Hercules, CA, USA). To isolate proteins from mitochondria, mitochondrial fractions were enriched using the Mitochondria Isolation Kit for Cultured Cells, as per the manufacturer's guidelines (Thermo Fisher Scientific, Waltham, MA, USA). Following isolation of mitochondria, proteins from mitochondrial fractions were extracted using RIPA buffer, as described above. Equal amounts of protein $(5-25 \mu \mathrm{g})$ were resolved using SDS polyacrylamide gel electrophoresis (SDSPAGE) and NuPAGE ${ }^{\circledR} 4-12 \%$ bis-tris precast gels (Invitrogen, Carlsbad, CA, USA). For immunoblotting of LC3B/MAPLC3B, $30 \mu \mathrm{g}$ of total protein was resolved on either 4-12\% bis-tris or $18 \%$ tris-glycine gels. Following electrophoresis, proteins were transferred onto nitrocellulose membrane (Bio-Rad, Hercules, CA, USA) using either the XCell II SureLock ${ }^{\mathrm{TM}}$ wet transfer or Invitrogen iBlot $^{\circledR} 2$ dry transfer systems (Invitrogen, Carlsbad, CA, USA). Wet transfer was performed under constant voltage $(30 \mathrm{~V})$ for $2 \mathrm{~h}$ at room temperature, with pre-chilled transfer buffer $(25 \mathrm{mM}$ Tris Base, $190 \mathrm{mM}$ Glycine [Sigma-Aldrich, St. Louis, MO, USA], 20\% Methanol [Millipore, Billerica, MA, USA]). For detection of MyHC and MyLC, wet transfer was performed using the Trans-Blot ${ }^{\circledR}$ cell module (Bio-Rad, Hercules, CA, USA) at $70 \mathrm{~V}$ for 2.5 hours at $4^{\circ} \mathrm{C}$. To confirm equal loading of samples, membranes were stained with Ponceau $\mathrm{S}$ solution (Sigma-Aldrich, St. Louis, MO, USA). After removing excess Ponceau S stain with TBS-T, membranes were blocked for 1 hour at room temperature and hybridized with specific primary antibodies overnight at $4^{\circ} \mathrm{C}$. Details of primary antibodies' sources, catalogue numbers, and dilutions are provided in Table 2.29. Following overnight incubation, primary antibodies were removed and membranes were incubated with respective horseradish peroxidase (HRP) conjugated secondary antibodies for $1 \mathrm{~h}$ at room temperature. Details of secondary antibodies are provided in Table 2.30. Immuno-reactive bands were detected using Western Lightning ${ }^{\circledR}$ Plus-enhanced 
chemiluminescence (ECL) reagent (Perkin Elmer, Waltham, MA, USA) and either autoradiography films or the ChemiDoc ${ }^{\mathrm{TM}}$ Touch Imaging System (Bio-Rad, Hercules, CA, USA). To quantify changes in protein levels, densitometry analysis was performed using either the GS-800 calibrated densitometer or Image Lab ${ }^{\mathrm{TM}}$ Software (Bio-Rad, Hercules, CA, USA).

\subsubsection{RNA Extraction and Real Time Quantitative PCR (qPCR)}

To extract RNA, cells were washed once with $10 \mathrm{ml}$ ice-cold PBS solution after which $1 \mathrm{ml}$ TRIzol ${ }^{\circledR}$ reagent was added per $10 \mathrm{~cm}^{2}$ plate (Thermo Fisher Scientific, Waltham, MA, USA). For RNA isolation from tissue, approximately $30 \mathrm{mg}$ of skeletal muscle tissue was homogenized with $1 \mathrm{ml} \mathrm{TRIzol}{ }^{\circledR}$ reagent using the Qiagen Tissue Lyser-II instrument, as described in Section 2.2.6. Following homogenization, $200 \mu 1$ chloroform, per $1 \mathrm{ml}$ of TRIzol ${ }^{\circledR}$ originally used, was added and tubes were shaken vigorously for 15 seconds. Following incubation for 3 minutes at room temperature, tubes were centrifuged at $12000 \mathrm{xg}$ for 15 minutes at $4^{\circ} \mathrm{C}$ and the upper aqueous phase was transferred into fresh micro centrifuge tubes. RNA was precipitated by adding 500 $\mu 1$ ice-cold isopropanol, per $1 \mathrm{ml}$ TRIzol ${ }^{\circledR}$ originally used, within 10 minutes at room temperature. Subsequently, samples were centrifuged at $12000 \mathrm{xg}$, for 10 minutes, at $4^{\circ} \mathrm{C}$. Pellets were then washed with $1 \mathrm{ml}$ ice-cold $75 \%$ ethanol, per $1 \mathrm{ml}$ TRIzol $^{\circledR}$ originally used, and recollected by spinning at 7500xg, for 5 minutes. The $75 \%$ ethanol was then removed and pellets were allowed to air dry for 5-10 minutes. The resulting pellets were resuspended in 30-50 $\mu$ DEPC water ( $1^{\text {st }}$ Base, Singapore) and RNA concentration was estimated using the NanoDrop ${ }^{\mathrm{TM}} 1000$ spectrophotometer (Thermo Fisher Scientific, Waltham, MA, USA). RNA electrophoresis was performed using 250$500 \mathrm{ng}$ of each RNA to confirm RNA integrity.

To generate complementary DNA (cDNA), $1 \mu \mathrm{g}$ RNA was reverse transcribed using $4 \mu \mathrm{l} 5 \mathrm{X} \mathrm{iScript}^{\mathrm{TM}}$ reaction mix (Bio-Rad, Hercules, CA, USA) and $1 \mu 1$ iScript $^{\mathrm{TM}}$ reverse transcriptase enzyme (Bio-Rad, Hercules, CA, USA), using the following conditions: $25^{\circ} \mathrm{C}$ for 5 minutes, $42^{\circ} \mathrm{C}$ for 30 minutes, and $85^{\circ} \mathrm{C}$ for 5 minutes. Prior to qPCR, cDNA samples were diluted 1:5 through the addition of MQ water. For qPCR analysis, $3 \mu \mathrm{l}$ of relevant cDNA, $1 \mu \mathrm{l}$ of $2.5 \mu \mathrm{M}$ gene specific forward primer, $1 \mu 12.5$ $\mu \mathrm{M}$ of gene specific reverse primer and $7 \mu$ of SsoFast ${ }^{\mathrm{TM}}$ EvaGreen $^{\circledR}$ Supermix was loaded per well into Hard-Shell ${ }^{\circledR}$ 96-well PCR plates. qPCR analysis was performed 
using the CFX96 Touch ${ }^{\mathrm{TM}}$ Real-Time PCR detection system (Bio-Rad, Hercules, CA, USA). qPCR conditions were as follows: $98^{\circ} \mathrm{C} 3$ minutes, 40 cycles of denaturation, annealing and elongation at $98^{\circ} \mathrm{C}$ for 15 seconds, $55-60^{\circ} \mathrm{C}$ for 10 seconds and $72^{\circ} \mathrm{C}$ for 20 seconds, respectively, followed by a melting curve from $65^{\circ} \mathrm{C}$ to $95^{\circ} \mathrm{C}$ in 10 seconds increments of $0.5^{\circ} \mathrm{C}$. Gene names and primer sequences are provided in Table 2.31. Gene expression was analysed using the $\Delta \Delta \mathrm{C}_{\mathrm{t}}$ method and represented as fold change, normalised to the expression of either Gapdh or Ppia.

\subsubsection{Assessment of 20S Proteasome Activity}

To determine 20S proteasome activity, C2C12 cells were seeded into clear bottom 96-well black plates at a density of 15000 cells per $\mathrm{cm}^{2}$ and maintained and differentiated as described in Section 2.2.2. Fully differentiated myotubes were transfected as previously described (Section 2.2.3). A volume of $100 \mu$ of the Proteasome-Glo ${ }^{\mathrm{TM}}$ cell-based reagent, containing a specific peptide substrate Succinylleucine-leucine-valine-tyrosine-aminoluciferin (Suc-LLVY-aminoluciferin), Z-leucinearginine- arginine-aminoluciferin (Z-LRR-aminoluciferin) and Z-norleucine-prolinenorleucine-aspartate-aminoluciferin (Z-nLPnLD-aminoluciferin) was added into wells containing the cells for the assessment of chymotrypsin-like, trypsin-like and caspaselike activities, respectively. 96-well plate was then shaken on a plate shaker for 2 minutes at $700 \mathrm{xrpm}$ and incubated at room temperature for 10 minutes. The plate then was read using the GloMax ${ }^{\circledR} 96$ microplate luminometer and the Proteasome-Glo programme that was pre-installed by manufacturer (Promega, Madison, WI, USA). Relative light units (RLU) detected by the machine were normalized to total protein and represented as percentage of values obtained from control-siRNA transfected cells.

\subsubsection{Detection of Nascent Protein Synthesis}

To determine nascent protein synthesis, C2C12 myoblasts were seeded at a density of 10000 cell $/ \mathrm{cm}^{2}$ into 96-well clear bottom black plates, maintained and differentiated as described in Section 2.2.2. Following $72 \mathrm{~h}$ differentiation, cells were incubated with methionine-free RPMI medium (Thermo Fisher Scientific, Waltham, MA, USA), supplemented with $50 \mu \mathrm{M}$ of methionine analog, Click-iT ${ }^{\circledR}$ homopropargylglycine (HPG) for 30 minutes. Following incubation at $37^{\circ} \mathrm{C}$ in a $5 \%$ $\mathrm{CO}_{2}$ incubator, medium was removed and cells were washed once with $100 \mu \mathrm{PBS} /$ well. 
Cells were then fixed with $100 \mu \mathrm{l} /$ well of $3.7 \%$ formaldehyde solution in PBS for 15 minutes at room temperature. Cell were then washed twice with $3 \%$ BSA solution in PBS and permeabilized with $100 \mu \mathrm{l} /$ well of $0.5 \%$ Triton ${ }^{\circledR} \mathrm{X}-100$ for 20 minutes at room temperature. Permeabilization solution was removed and cells were washed twice with $100 \mu \mathrm{l} /$ well 3\% BSA. $100 \mu \mathrm{l} /$ well of Click-iT ${ }^{\circledR}$ reaction cocktail was added to each well and the plate was incubated in the dark for 30 minutes at room temperature. After incubation, each well was washed with $100 \mu$ Click-iT $^{\circledR}$ reaction rinse buffer. To stain nuclear components, NuclearMask ${ }^{\mathrm{TM}}$ Blue Stain was diluted 1:2000 in PBS. $100 \mu \mathrm{l} /$ well of diluted NuclearMask ${ }^{\mathrm{TM}}$ Blue Stain solution was added to each well and the plate was incubated in the dark for 30 minutes at room temperature. The solution containing the NuclearMask $^{\mathrm{TM}}$ Blue Stain was then removed and cells were washed twice with $100 \mu \mathrm{l}$ PBS/well, before a final volume of $100 \mu \mathrm{l}$ of PBS was added to each well. Fluorescent intensity was measured from 8 wells with appropriate filters for Alexa Fluor ${ }^{\circledR} 488$ (Excitation/Emission: $488 \mathrm{~nm} / 520 \mathrm{~nm}$ ) and NuclearMask ${ }^{\mathrm{TM}}$ Blue Stain (Excitation/Emission: $355 \mathrm{~nm} / 460 \mathrm{~nm}$ ) using the Tecan 2000 fluorescent plate reader and iControl software (Tecan Group Ltd, Männedorf, Switzerland). Mean fluorescence intensity (MFI) of Alexa Fluor ${ }^{\circledR} 488$ was normalized to MFI of DNA content that is stained by NuclearMask ${ }^{\mathrm{TM}}$ Blue Stain to obtain nascent protein synthesis. MFI values were plotted as bar graph with error bars indicating \pm s.e.m. 


\subsubsection{Assessment of Mitochondrial Respiratory Capacity}

C2C12 myoblasts and primary myoblasts were seeded at the density of 15000 cell per $\mathrm{cm}^{2}$ onto $\mathrm{XF}^{\mathrm{e}} 24$ cell culture microplates (Agilent Technologies, Santa Clara, $\mathrm{CA}$, USA). $\mathrm{XF}^{\mathrm{e}} 24$ sensor cartridges were hydrated overnight at $37^{\circ} \mathrm{C}$ in a non- $\mathrm{CO}_{2}$
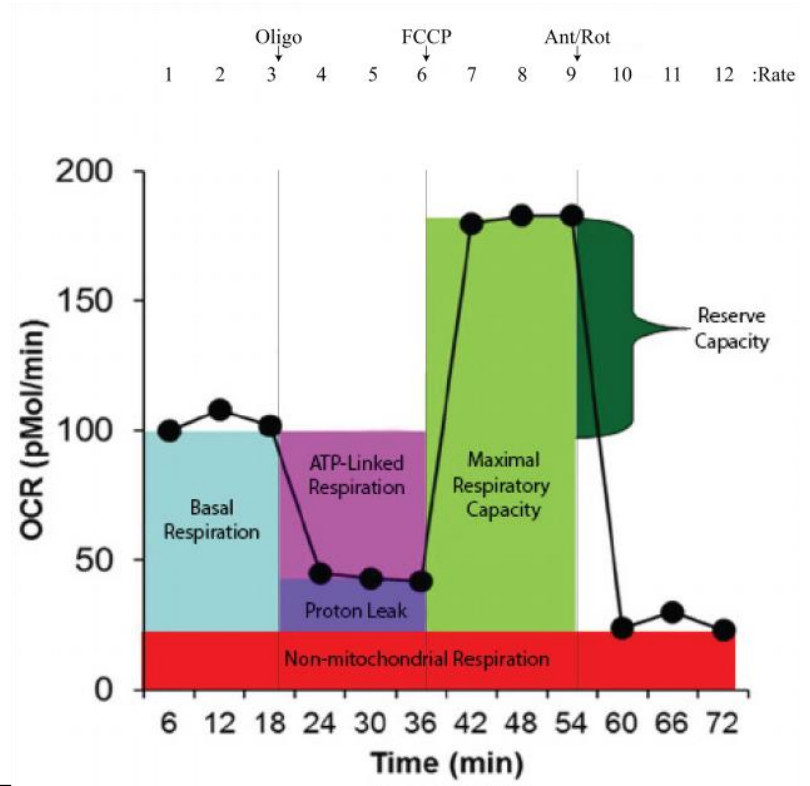

Figure 2-1: Representation of mitochondrial respiration parameters.

Areas representing basal respiration, ATPlinked respiration (ATP production), maximum respiration, proton leak, nonmitochondrial respiration and spare respiratory capacity are indicated with different colours. The time points when oligomycin, FCCP and antimycin A \& rotenone were injected are indicated with arrows. OCR measurement and rates are indicated with black dots and lines respectively (Translational Psychiatry, 2014 Apr; 4(4): e377.; doi: 10.1038/tp.2014.15). Permission to use this copyrighted image is granted by RightsLink online copyright clearance centre (See Appendix 6.6.).

when drugs injected. For the MitoStress Test Kit, basal respiration was recorded at rate 3 , just prior to oligomycin injection, maximum respiration was calculated as the maximum OCR during rate 7,8 and 9 and proton leak was calculated at rate 6. Spare respiratory capacity and ATP-linked respiration were obtained by subtracting basal respiration from maximum respiration and proton leak values, respectively. Nonmitochondrial respiration (minimum OCR during rate 10, 11 and 12) was subtracted from all respiratory calculations. Each respiration parameter is represented with area highlighted with different colour in Figure 2.1. incubator by adding $1 \mathrm{ml} /$ well $\mathrm{XF}$ calibrant solution into the utility plate. Medium was replaced with Assay Medium (for Seahorse XF Cell Mito Stress Test Kit see Table 2.24 and see Table 2.25 for Seahorse XF Glycolysis Stress Test Kit) and microplates were incubated at $37^{\circ} \mathrm{C}$ in a non- $\mathrm{CO}_{2}$ incubator for 30 minutes. Three measurements were recorded before and after sequential injection of $1 \mu \mathrm{M}$ Oligomycin, $0.5 \mu \mathrm{M}$ FCCP and $0.5 \mu \mathrm{M}$ Antimycin/Rotenone solutions (Agilent Technologies, Santa Clara, CA, USA). Protein concentration was estimated for each well containing cells using Bio-Rad Protein Assay reagent (Bio-Rad, Hercules, CA, USA). Figure 2.1 highlights the typical schematic representation of real-time OCR measurement with rates, time points 


\subsubsection{Measurement of Exogenous Fatty Acid Oxidation}

To determine exogenous fatty acid oxidation (FAO), myoblasts were grown and differentiated in $\mathrm{XF}^{\mathrm{e}} 24$ cell culture microplates, as described in Section 2.2.10. Cells were subjected to glycolytic stress and induced to catabolise fatty acids by replacing growth medium with Substrate-limited Medium (see Table 2.26) supplemented with 0.5 $\mathrm{mM}$ glucose, $1 \mathrm{mM}$ glutamine and $0.5 \mathrm{mM}$ carnitine and 1\% FBS for $6 \mathrm{~h}$. Subsequently, the substrate-limited medium was changed to FAO Assay Medium (see Table 2.27) 30 minutes prior to beginning the assay run. Palmitate-BSA conjugation was carried out as follows: The required amount of BSA was dissolved in $150 \mathrm{mM} \mathrm{NaCl}$ solution in a $37^{\circ} \mathrm{C}$ water bath. Palmitate was dissolved in $150 \mathrm{mM} \mathrm{NaCl}$ solution at $70^{\circ} \mathrm{C}$ while stirring. Once the palmitate solution became clear, the palmitate- $\mathrm{NaCl}$ solution was added to the BSA-NaCl solution dropwise $87.5 \mu \mathrm{l} /$ well Palmitate-BSA FAO substrate was added onto the cell and cells were incubated in non- $\mathrm{CO}_{2}$ incubator at $37^{\circ} \mathrm{C}$ for 15 minutes prior to assay run. $1 \mu \mathrm{M}$ Oligomycin, $0.5 \mu \mathrm{M}$ FCCP and $0.5 \mu \mathrm{M}$ Antimycin/Rotenone solutions were prepared as described in Section 2.2.10 and the plate was loaded into XFe24 Extracellular Flux Analyzer (Agilent Technologies, Santa Clara, CA, USA). Quantification of OCR from exogenous fatty acid oxidation was calculated as outlined in Section 2.2.10.

\subsubsection{MitoTracker Red CMXRos Staining and Confocal Microscopy}

C2C12 myoblasts were seeded on 8-well glass chamber slides (Thermo Fisher Scientific, Waltham, MA, USA) at the density of 5000 cells/well. Cells were stained with $200 \mathrm{nM}$ MitoTracker ${ }^{\circledR}$ Red CMXRos for 20 minutes at $37^{\circ} \mathrm{C}$ in a $5 \% \mathrm{CO}_{2}$ incubator following overnight attachment (Thermo Fisher Scientific, Waltham, MA, USA). Cells were washed with proliferation medium followed by fixation with $2 \%$ paraformaldehyde for 15 minutes and $4 \%$ paraformaldehyde for 10 minutes at $37^{\circ} \mathrm{C}$. Slides were mounted with SlowFade ${ }^{\circledR}$ Gold Antifade Mountant with DAPI (Thermo Fisher Scientific, Waltham, MA, USA) and covered with glass coverslips (VWR, Radnor, PA. USA). MitoTracker ${ }^{\circledR}$ stained myoblasts were visualized using the Olympus BX61 WI microscope and analyzed using the Olympus FV1000 viewer software (Olympus Imaging, Central Valley, PA, USA). 


\subsubsection{FACS Analysis of MitoTracker ${ }^{\circledR}$ Green FM Stained Myoblasts}

C2C12 myoblasts and murine primary myoblasts were seeded at a density of 15000 cells per $\mathrm{cm}^{2}$ onto 6-well plates. The next day, myoblasts were stained with 150 nM MitoTracker ${ }^{\circledR}$ Green FM (Thermo Fisher Scientific, Waltham, MA, USA) for 20 minutes at $37^{\circ} \mathrm{C}$ in the dark, trypsinized and collected in conical tubes. Cell pellets from three biological replicates were resuspended in PBS and FACS analysis was performed immediately using the FITC channel of the FACSCanto flow cytometry system (BD Biosciences, Franklin Lakes, NJ, USA). 10000 events from each of 3 biological replicates were recorded and subtracted from background signal recorded from unstained cells. A total of 10,000 events from 3 replicate wells per experimental group were detected using the FITC channel. Fluorescence intensity was averaged and represented as mean fluorescence intensity $(\mathrm{MFI}) \pm$ s.e.m.

\subsubsection{Assessment of Cellular ROS Production}

To assess cellular ROS production in $\mathrm{C} 2 \mathrm{C} 12$ myoblasts, $\mathrm{C} 2 \mathrm{C} 12$ cells were seeded onto 96-well clear bottom black microplates at a density of 10000 cells/well and were transiently transfected next day with the DharmaFECT 1 transfection reagent (Dharmacon RNAi Technologies, Lafayette, CO, USA), as described in Section 2.2.3. 24 hours' post-transfection, cells were stained with $10 \mu \mathrm{M} \mathrm{H}_{2}$ DCFDA for 30 minutes as per manufacturer's instruction (Thermo Fisher Scientific, Waltham, MA, USA). To assess cellular ROS generation in primary myotubes, primary myotubes were isolated from hind limb muscle of Parkin KO mice and age-matched wild type controls, as described in Section 2.2.4. $72 \mathrm{~h}$ differentiated primary myotubes were stained with 10 $\mu \mathrm{M} \mathrm{H}_{2}$ DCFDA for 30 minutes as per manufacturer's guidelines. The $\mathrm{H}_{2}$ DCFDA stain was then removed and cells were washed once with $200 \mu \mathrm{PBS} /$ well, before a final volume of $100 \mu \mathrm{l}$ of PBS was added to each well. Fluorescent intensity (Excitation/Emission: $492-495 \mathrm{~nm} / 517-527 \mathrm{~nm}$ ), which is an indication of ROS levels, was measured in quadruplicate using the Tecan 2000 fluorescent plate reader and iControl software (Tecan Group Ltd, Männedorf, Switzerland).

\subsubsection{Genomic DNA Isolation and mtDNA Copy Number Analysis}

To determine mtDNA copy number, genomic DNA was extracted using the Q1Aamp ${ }^{\circledR}$ DNA Mini Kit, according to the manufacturer's instructions (Qiagen, 
Valencia, CA, USA). To determine mtDNA copy number qPCR was performed using $5 \mathrm{ng} / \mu \mathrm{l}$ genomic DNA, together with $1 \mu \mathrm{l}$ of $2.5 \mu \mathrm{M}$ mitochondrial gene ( $\mathrm{mtCoI})$ and nuclear gene $(18 S)$ specific primers and $10 \mu \mathrm{l}$ SsoFast EvaGreen Supermix per reaction (Bio-Rad, Hercules, CA, USA). The qPCR conditions were as follows: $95^{\circ} \mathrm{C}$ for 5 minutes, 45 cycles of $95^{\circ} \mathrm{C}$ for 10 seconds, $60^{\circ} \mathrm{C}$ for 20 seconds, $72^{\circ} \mathrm{C} 20$ seconds followed by melting curve. Details of primers used to determine mtDNA copy number are provided in Table 2.31. Results were analysed using the $\Delta \Delta \mathrm{C}_{\mathrm{t}}$ method and resulting data was represented as fold change, relative to respective controls.

\subsubsection{Hematoxylin and Eosin (H\&E) Staining and Myotube Area Analysis}

For H\&E staining of CCCP treated myotubes, C2C12 myoblasts were seeded and differentiated on Thermanox ${ }^{\mathrm{TM}}$ coverslips (Nunc, Rochester, NY, USA) at a density of 20000 myoblasts $/ \mathrm{cm}^{2}$. After $72 \mathrm{~h}$ differentiation, myotubes were treated with 1,5 , and $10 \mu \mathrm{M} \mathrm{CCCP}$ for $24 \mathrm{~h}$. Control myotubes were treated with equal volume of DMSO for $24 \mathrm{~h}$. For H\&E staining of transfected myotubes, myoblasts were seeded at the density of 12000 cells $/ \mathrm{cm}^{2}$ and differentiated on 2 or 4-well tissue culture treated $\mu$ Slides (Ibidi GmbH, Martinsried, Germany). Transfection was performed as described in Section 2.2.3. Following treatments and relevant incubation times all myotubes were fixed with 20:2:1 ethanol:formaldehyde:acetic acid solution for 30 seconds at room temperature. H\&E staining was performed as outlined in the following table (Table 2.36)

Table 2.36: Myotube H\&E Staining Protocol

$\begin{array}{cc}\text { Reagent } & \text { Exposure Time } \\ \text { Hematoxylin, Gill III } & 2 \text { minutes } \\ 0.1 \% \mathrm{HCl} & 2 \text { seconds } \\ \text { Scott's tap Water } & 1 \text { minute } \mathrm{x} 5 \\ \text { Eosin-Y } & 2 \text { minutes } \\ 100 \% \text { Ethanol } & 5 \text { minutes } \times 3 \\ \text { Xylene } & 5 \text { minutes } \times 2 \\ \text { DPX mounting } & \end{array}$

For staining of transfected myotubes on $\mu$-Slides, xylene and DPX mounting were avoided and images were captured immediately following ethanol dehydration. Myotubes were imaged using the Leica DM6000 B microscope (Leica Microsystems, Wetzlar, Germany), equipped with 10X objective and the area of individual myotubes was determined using ImageJ software (Bethesta, MA, USA). For CCCP treatment the 
area of 10 myotubes per image ( $n=5$, per coverslip) across 2-3 biological replicates per treatment group. For Parkin knockdown experiments the area of 200 myotubes was measured across 4 biological replicates each for Control-siRNA and Parkin-siRNA transfected myotubes. Results were represented as average myotube area.

\subsubsection{H\&E Staining of Tibialis Anterior (TA) Muscle and Cross-Sectional Area Analysis}

Transverse serial sections $(10 \mu \mathrm{m})$ were cut from the mid-belly region of snap frozen OCT embedded TA muscles using the Leica CM1950 Cryostat (Leica Microsystems, Wetzlar, Germany). Sections were mounted on Superfrost ${ }^{\mathrm{TM}}$ glass microscope slides (Fisher Scientific, Waltham, MA, United States) and stored at $-80^{\circ} \mathrm{C}$ prior to $\mathrm{H} \& \mathrm{E}$ staining. The procedure for $\mathrm{H} \& \mathrm{E}$ staining of skeletal muscle sections is provided in Table 2.37 below:

\section{Table 2.37: H\&E Staining Protocol of Muscle Sections}

$\begin{array}{cc}\text { Reagent } & \text { Exposure Time } \\ \text { Hematoxylin, Gill III } & 1 \text { minute } \\ \text { Rinse with tap water } & \text { Until clear } \\ \text { Blue with Scott's tap water } & 2 \text { minutes } \\ \text { Rinse with tap water } & \text { Until clear } \\ \text { Eosin-Y } & 2 \text { minutes } \\ \text { Rinse with tap water } & \text { Until clear } \\ 50 \% \text { ethanol } & 3 \text { dips } \\ 70 \% \text { ethanol } & 3 \text { dips } \\ 95 \% \text { ethanol } & 2 \text { minutes } \\ 100 \% \text { ethanol } & 2 \text { minutes } \times 2 \\ \text { Xylene } & 5 \text { minutes } \times 2 \\ \text { DPX mounting } & \end{array}$

Images were captured as indicated in Section 2.2.16. Cross-sectional area of 1000 myofibers from each muscle section were recorded and represented as distribution graph. Entire TA muscle sections were imaged using the Leica DM6000 B microscope (Leica Microsystems, Wetzlar, Germany), equipped with 5X objective. For TA muscle sections, the area of 1000 myofibers were calculated from each muscle section per genotype using ImageJ software (Bethesta, MA, USA) and results were represented as a frequency distribution graph and average myotube area was also calculated. 


\subsubsection{Total RNA Isolation for Microarray and In Silico Analysis}

To identify global genes expression differences between wild type and Parkin KO mice, total RNA was isolated from Gas muscle of 3-month-old Parkin KO and agematched wild type controls using mirVANATM miRNA isolation kit, as per manufacturer's guidelines (Thermo Fisher Scientific, Waltham, MA, USA). RNA integrity was assessed using the Agilent RNA 6000 Nano Kit (Agilent Technologies, Santa Clara, CA, USA). Subsequent microarray analysis was performed by Genomax Technologies (Singapore) as per the following: $100 \mathrm{ng} / \mu \mathrm{l}$ RNA was probed with the Low Input AMP labelling Kit, One Color (Agilent p/n 5190-2305) as per manufacturer's instruction (One-color microarray-based gene expression, analysis low input quick amp labelling, version 6.5). $100 \mathrm{ng} / \mu \mathrm{l}$ RNA was converted into cDNA using oligo-dt primers containing a T7 RNA polymerase recognition site. Cyanine 3-CTP labelled cRNA was transcribed using T7 RNA polymerase. $600 \mathrm{ng}$ cRNA was hybridized onto Agilent SurePrint G3 Human GE 8x60K microarray slides at $65^{\circ} \mathrm{C}$, at $10 x r p m$ for 17 hours in an Agilent hybridization oven. In silico pathway analysis was performed with significantly upregulated ( $>1.5$ fold) and downregulated $(<0.75$ fold) genes with a $p$ value cut off $<0.05$ using Kyoto Encyclopaedia of Genes and Genomes (KEGG) pathway database integrated in the Database for Annotation, Visualization and Integrated Discovery (DAVID) tool (https://david.ncifcrf.gov). Protein class analysis and identification of significantly upregulated ubiquitin-conjugating enzyme, E3 ubiquitin ligases and deubiquitinating enzymes (DUBs) was carried out using Protein Analysis Through Evolutionary Relationships (PANTHER) gene list analysis software (http://www.pantherdb.org). Expression of significantly upregulated genes were validated using qPCR analysis, as previously described in Section 2.2.7.

\subsubsection{Statistical Analysis}

F-tests were performed on each data set to test for equal variance between groups. Two-tailed Student's T-test were conducted for statistical analysis to assess for differences between two groups, where the calculated F-value from the data sets is less than the F-critical value. Two-way ANOVA was applied for analysis of data from more than two groups, followed by Tukey post-hoc test. $* \mathrm{P}<0.05$, $* * \mathrm{P}<0.01$ and $* * * \mathrm{P}<0.001$ denote significance levels. Data was represented as mean \pm s.e.m. Outliers were removed following analysis of data using online GraphPad Software outlier calculator 
with an alpha $=0.05$ (GraphPad Software Inc., La Jolla, CA, USA). Researchers were not blinded during experiment plan and analysis. Details of biological replicates for each experiment are indicated in respective figure legends. 


\section{RESULTS}

Some parts from this section have been communicated with American Journal of Physiology-Cell Physiology Journal

\subsection{Treatment with CCCP promotes PINK1/PARKIN-mediated mitophagy in C2C12 myotubes}

To investigate if induction of mitophagy leads to atrophy of muscle cells, a model of PARKIN-mediated mitophagy was established through treating $\mathrm{C} 2 \mathrm{C} 12$ myotubes with CCCP. Previous studies have used $10 \mu \mathrm{M}$ CCCP for $24 \mathrm{~h}$ to induce PINK1/PARKIN-mediated mitophagy in different cell culture models ${ }^{113,266-268}$. Moreover, Gomez-Sanchez et al. has reported that only 24 h-10 $\mu \mathrm{M}$ CCCP treatment results in a significant reduction in mitochondrial content and 4-fold increase in PINK1 protein levels ${ }^{269}$. Therefore, in parallel to previous studies, $\mathrm{C} 2 \mathrm{C} 12$ myotubes were treated with $10 \mu \mathrm{M}$ CCCP for $24 \mathrm{~h}$. Quantitative real-time PCR (qPCR) analysis revealed increased mRNA expression of both Pinkl and Parkin following CCCP treatment (Fig. 3.1A). Subsequent immunoblot analysis revealed increased levels of full length PINK1 following $24 \mathrm{~h} \mathrm{CCCP}$ treatment in myotubes (Fig. 3.1B \& 3.2C). However, no observable difference in PARKIN levels was noted upon CCCP treatment (Fig. 3.1B \& 3.2C). Reduced levels of the mitochondrial fusion protein Mitofusin 2 (MFN2) and enhanced levels of the autophagosomal marker LC3-II (lipidated LC3) were also observed in response to CCCP treatment of $\mathrm{C} 2 \mathrm{C} 12$ myotube cultures (Fig. 3.1B \& 3.2C \& 3.2D). Despite no change in total PARKIN protein levels (Fig. 3.1C), treatment with CCCP resulted in increased levels of PARKIN protein in isolated mitochondrial fractions, which is consistent with both increased translocation of PARKIN to mitochondria and enhanced activation of PARKIN E3 ligase activity (Fig. $3.1 \mathrm{E} \& 3.2 \mathrm{~F})$. 
A

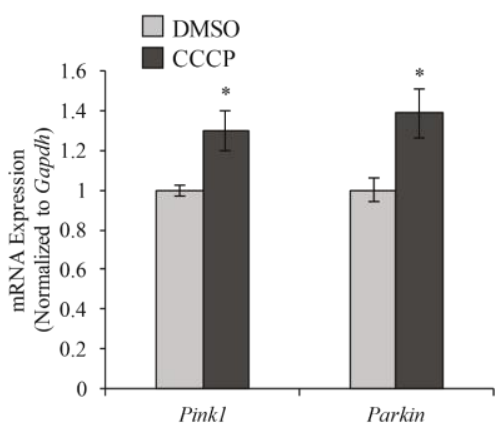

B

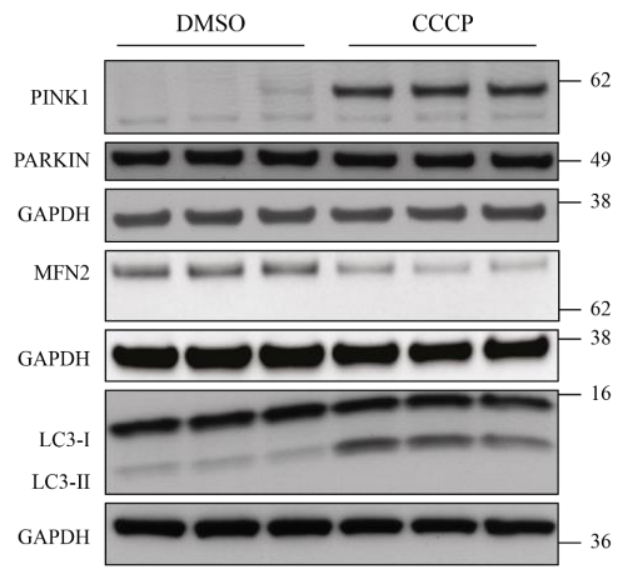

C

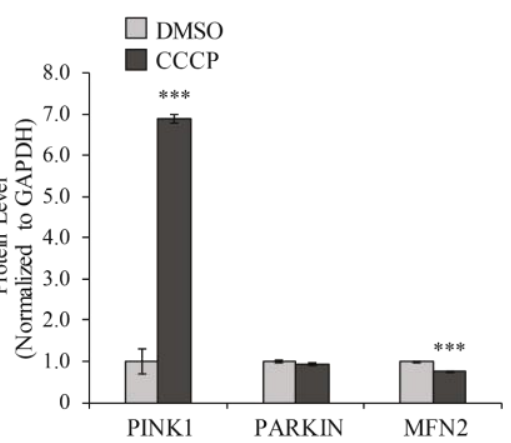

D

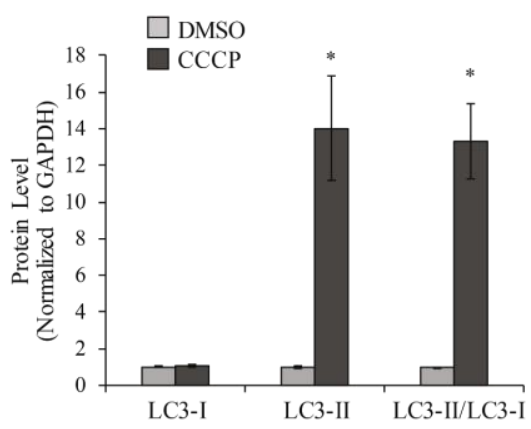

F

E

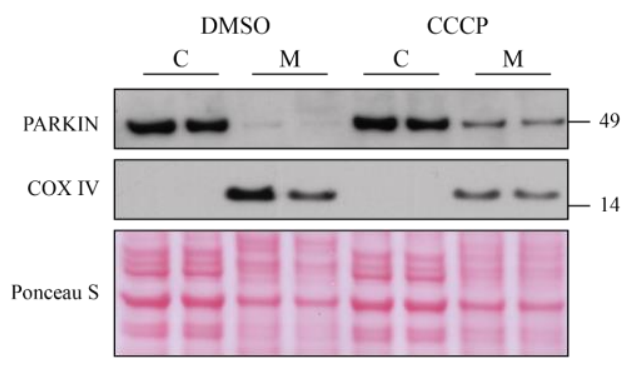

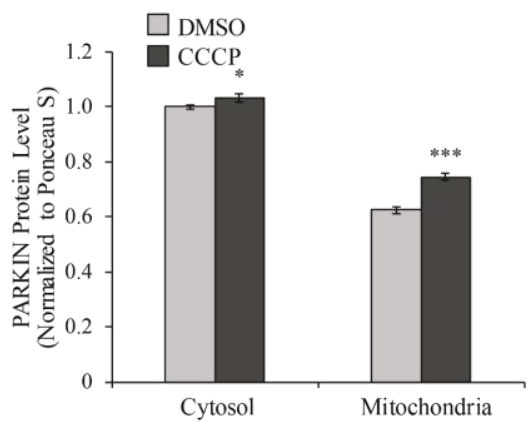

$G$

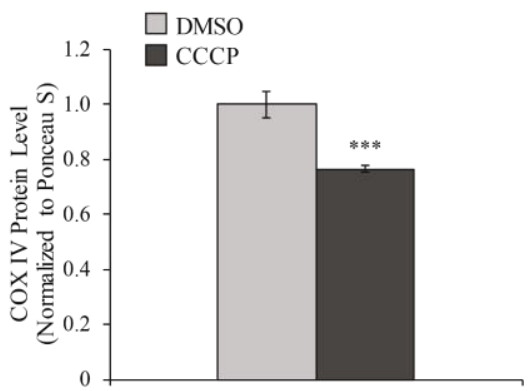


Figure 3.1: CCCP induces PINK1/PARKIN-mediated mitophagy in C2C12 myotubes A) Graph showing qPCR analysis of Pinkl and Parkin mRNA expression, normalized to Gapdh, in $24 \mathrm{~h}$ DMSO or $10 \mu \mathrm{M}$ CCCP treated myotubes $(\mathrm{n}=3)$. B) Representative images of western blot analysis of PINK1, PARKIN, MFN2, non-lipidated LC3 (LC3I) and lipidated LC3 (LC3-II) protein levels in DMSO and $10 \mu \mathrm{M}$ CCCP treated myotubes. The levels of GAPDH were assessed as a loading control $(n=3)$. C) Graph showing quantification of PINK1, PARKIN and MFN2 protein levels, normalized to GAPDH, in arbitrary units $(n=3)$. D) Graph showing quantification of LC3-I, LC3-II and LC3-II/LC3-I protein levels, normalized to GAPDH, in arbitrary units $(n=3)$. E) Representative images of western blot analysis of PARKIN and COX-IV protein levels in cytosolic (C) and mitochondrial (M) fractions isolated from myotubes treated with either DMSO or $10 \mu \mathrm{M}$ CCCP for $24 \mathrm{~h}$. COX IV protein levels were assessed to confirm purity of mitochondrial fraction and Ponceau-S staining was performed as a loading control $(\mathrm{n}=8)$. F) Graph showing quantification of PARKIN protein levels in DMSO and $10 \mu \mathrm{M}$ CCCP treated cytosolic and mitochondrial fractions. Protein levels are normalized to Ponceau $\mathrm{S}$, in arbitrary units $(\mathrm{n}=8) \mathbf{G})$ Graph showing quantification of COX IV protein levels, normalized to Ponceau $S$, in arbitrary units ( $n=8)$ Graph shows mean \pm s.e.m. $* \mathrm{P}<0.05$, $* * * \mathrm{P}<0.001$ (Student's t-test). 


\subsection{CCCP treatment leads to reduced mitochondrial mass and abnormal mitochondrial morphology in vitro}

Next, the effect of CCCP on the levels of mitochondrial oxidative phosphorylation (OXPHOS) proteins was determined. Immunoblotting of critical mitochondrial OXPHOS proteins revealed a significant reduction in the levels of mitochondrial complex proteins necessary for oxidative phosphorylation upon CCCP treatment (Fig. 3.2A \& 3.2B), with significantly lower levels of UQCRC2 (Complex III), MTCOI (Complex IV), SDHB (Complex II) and NDUFB8 (Complex I) noted upon CCCP treatment (Fig. 3.2A \& 3.2B). However, no significant difference in the Complex $\mathrm{V}$ protein ATP5 was observed following CCCP treatment (Fig. 3.2A \& 3.2B). Next, mitochondrial number was quantified through determining mitochondrial DNA (mtDNA) copy number and MitoTracker Green FM staining and FACS analysis. Results revealed a significant decrease in mtDNA copy number and MitoTracker Green FM intensity in myoblasts upon CCCP treatment (Fig. 3.2C \& 3.2D), which is indicative of decreased mitochondrial number. To visualize changes in mitochondrial architecture in response to CCCP treatment, MitoTracker Red CMXRos staining and confocal microscopy were performed (Fig. 3.2E). Subsequent assessment of mitochondria morphology revealed disruption of the typical filamentous mitochondria network, resulting in a punctate mitochondria morphology, which is consistent with mitochondria swelling and fragmentation, in response to CCCP treatment (Fig. 3.2E). Collectively, these data suggest that CCCP treatment promotes PINK1/PARKIN mediated mitophagy in vitro model of skeletal muscle. 


\subsection{CCCP treatment leads to impaired mitochondrial respiratory capacity and reduced utilization of exogenous fatty acids in vitro}

To investigate the effect of CCCP treatment on mitochondrial function, oxygen consumption rate (OCR) was assessed using the Seahorse $\mathrm{XF}^{\mathrm{e}} 24$ extracellular flux analyzer. A dramatic reduction in real-time oxygen consumption rate was noted in myotubes treated with CCCP, when compared to vehicle (DMSO) treated controls (Fig. 3.3A). Indices of mitochondrial function were calculated and a significant reduction in basal, maximal, ATP-linked and non-mitochondrial respiration was noted upon treatment with CCCP (Fig. 3.3B). In addition, a significant reduction in spare respiratory capacity was further observed in CCCP treated myotubes, when compared to vehicle controls (Fig. 3.3B). Given that mitochondria are the major site for fatty acid oxidation, real time utilization of exogenous fatty acids (palmitate) was next measured using the Seahorse $\mathrm{XF}^{\mathrm{e}} 24$ analyzer. Real time OCR measurements revealed a dramatic reduction in OCR in myotubes treated with CCCP, in the presence of either BSA or palmitate (Fig. 3.3C \& 3.3D). Moreover, spare and maximal respiration, due to utilization of palmitate, were also significantly reduced in CCCP treated myotubes (Fig. 3.3D). Taken together, these data suggest that CCCP treatment leads to reduced mitochondrial respiration and respiration due to utilization of exogenous fatty acids in $\mathrm{C} 2 \mathrm{C} 12$ myotubes.

\subsection{CCCP treatment activates the energy sensor AMPK $\alpha$ in vitro}

AMPK is key energy sensor and regulator in cells, in fact, AMPK is activated in response to reduced levels of ATP and functions to switch off anabolic (energy utilizing)

processes in favor of catabolic (energy producing) processes 270,271. To determine whether AMPK is activated in response to CCCP treatment, the levels of active phosphorylated AMPK $\alpha$ (p-AMPK $\alpha$ ) were quantified. Consistent with reduced mitochondrial respiratory capacity, western blot analysis revealed increased levels of p$\mathrm{AMPK} \alpha$ in $\mathrm{C} 2 \mathrm{C} 12$ myotubes upon treatment with CCCP (Fig. 3.3E \& 3.3F). 
A

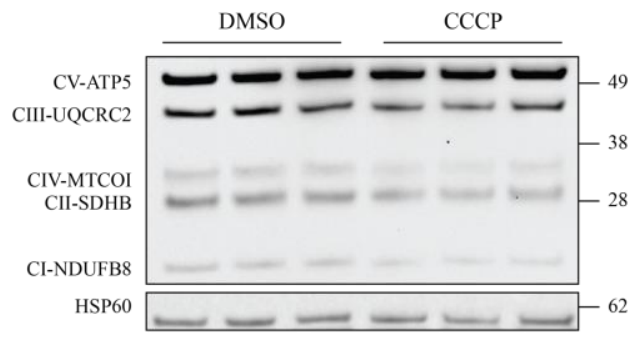

C

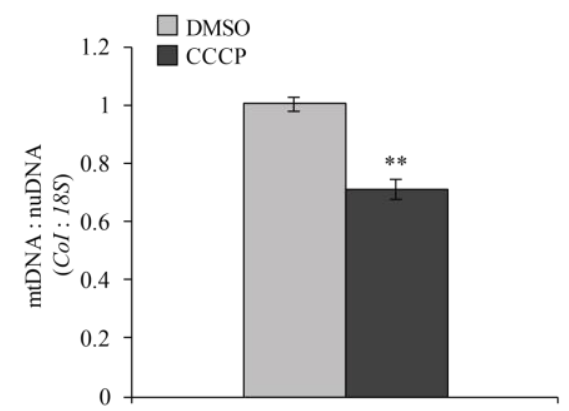

B

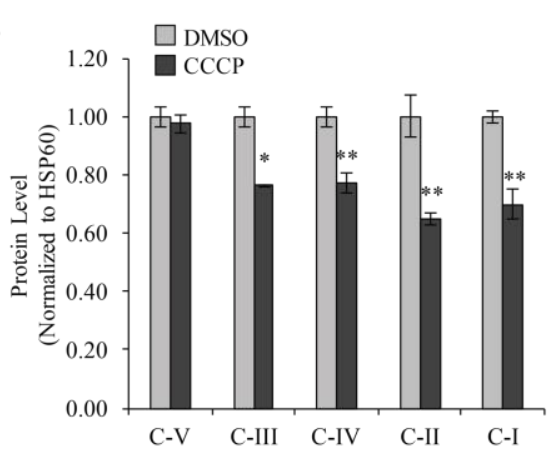

D

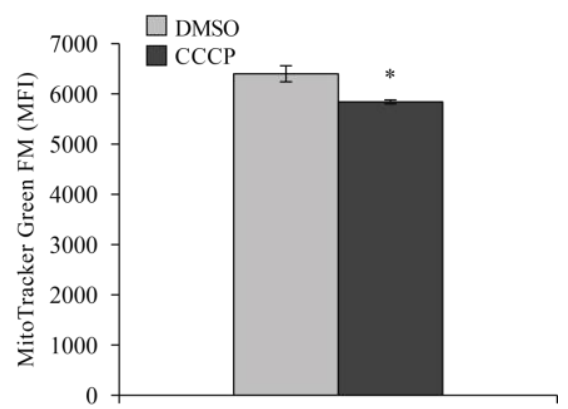

E

DMSO
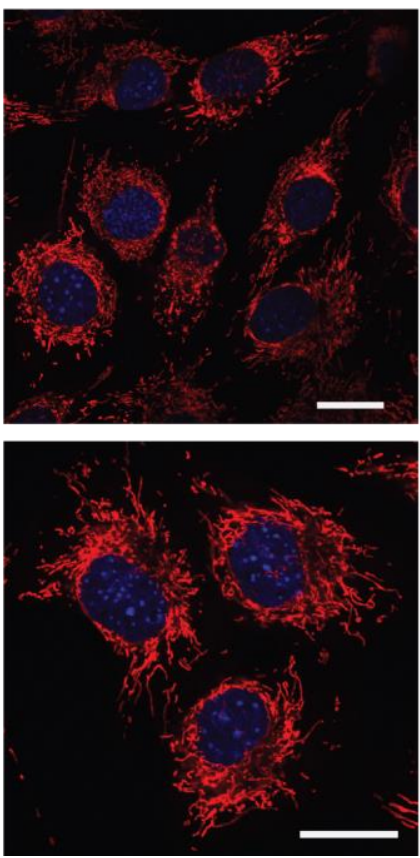

CCCP
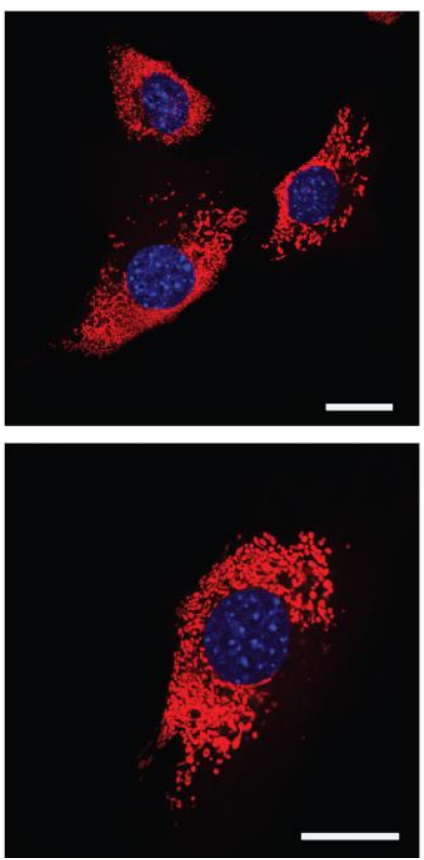
Figure 3.2: CCCP treatment leads to reduced mitochondrial mass and fragmented mitochondrial network

A) Representative images of western blot analysis of OXPHOS subunit (ATP5, UQCRC2, MTCOI, SDHB and NDUFB8) protein levels in C2C12 myotubes treated with either DMSO or $10 \mu \mathrm{M}$ CCCP for $24 \mathrm{~h}$. The levels of HSP60 were assessed as a loading control $(n=3)$. B) Graph showing quantification of OXPHOS protein levels, normalized to HSP60, in arbitrary units $(n=3)$. C) Graph showing qPCR analysis of mitochondrial DNA (mtDNA) copy number:nuclear DNA (nuDNA) copy number ratio in myotubes treated with either DMSO or $10 \mu \mathrm{M}$ CCCP for $24 \mathrm{~h}$. Values are expressed as fold change relative to DMSO control. The expression of the mitochondrial gene CoI was normalized to the nuclear gene $18 S(\mathrm{n}=3)$. D) Graph showing results of FACS analysis of MitoTracker Green FM stained myoblasts following $24 \mathrm{~h}$ treatment with either DMSO or $10 \mu \mathrm{M}$ CCCP (MFI: Mean fluorescence intensity) (n=3). E) Representative confocal images of MitoTracker Red CMXRos stained myoblasts treated with either DMSO or $10 \mu \mathrm{M}$ CCCP for $24 \mathrm{~h}$. Dapi (blue) was used to stain nuclei. Images in the top panels reflect cells captured using the 100X objective and images in the bottom panels are magnified further 1.5X and captured using the Olympus BX61 WI confocal microscope. Scale bars represent $20 \mu \mathrm{m}$. All graphs show mean \pm s.e.m. $* \mathrm{P}<0.05, * * \mathrm{P}<0.01$ (Student's t-test). 


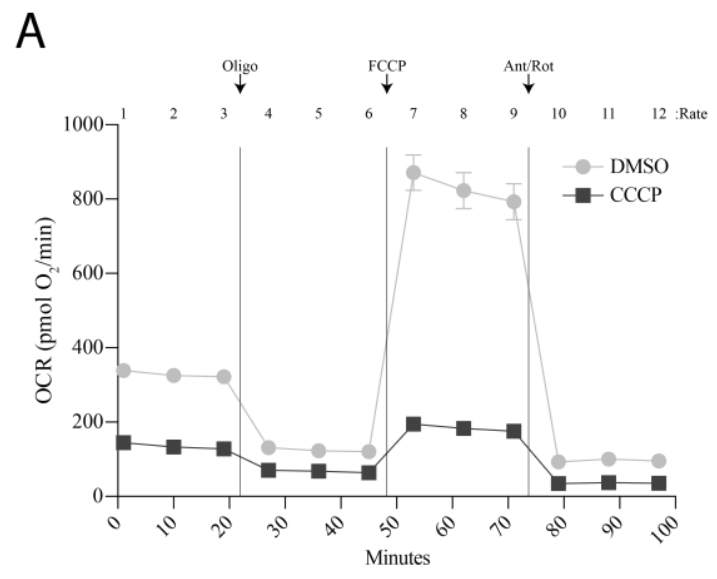

C

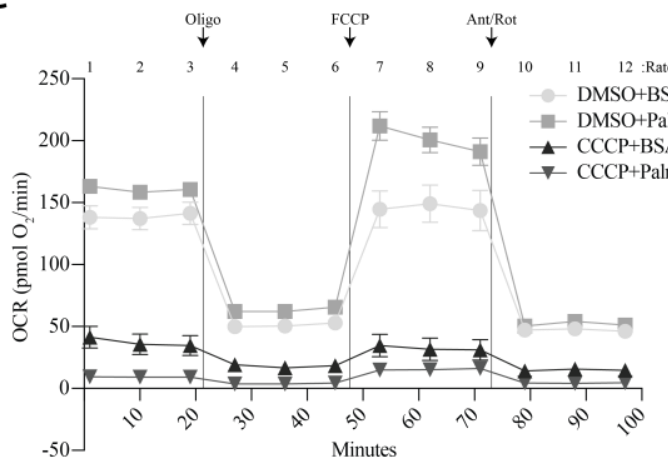

D

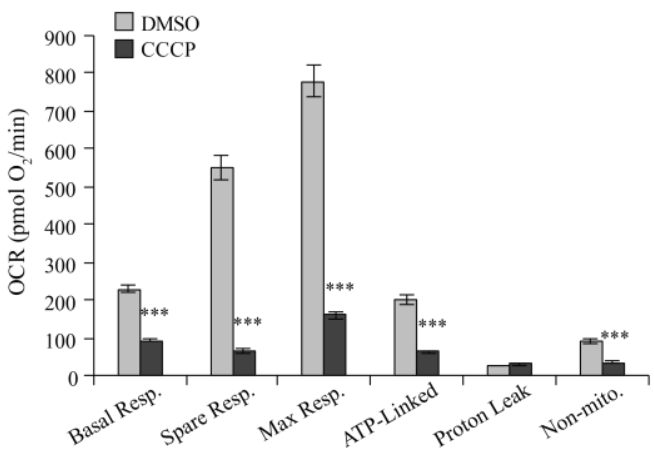

E

F
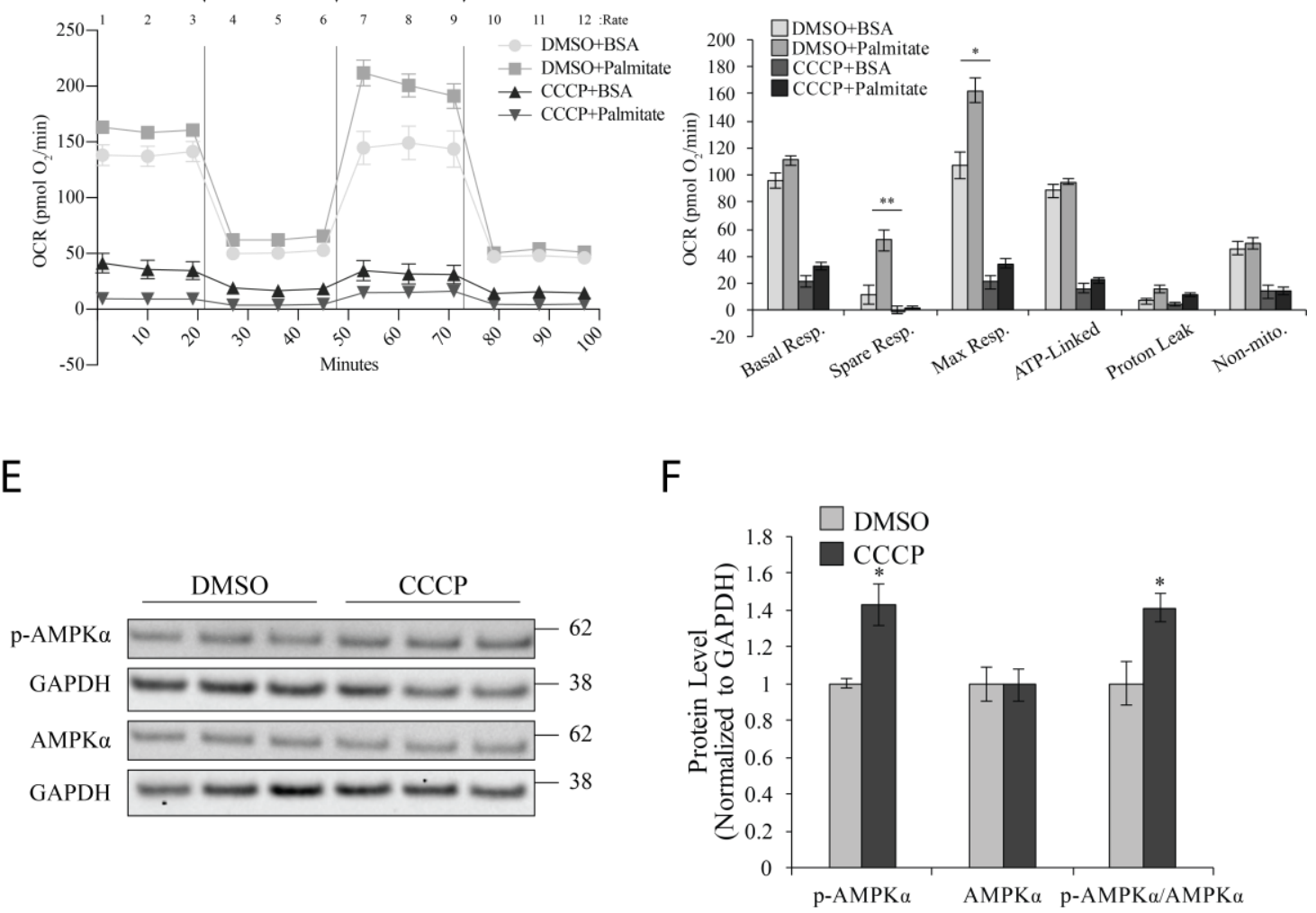
Figure 3.3: CCCP treatment results in mitochondrial dysfunction in C2C12 myotubes.

A) Graph showing the real-time Oxygen Consumption Rate (OCR) of myotubes treated with either DMSO or $10 \mu \mathrm{M}$ CCCP for $24 \mathrm{~h}$, as assessed by the Seahorse $\mathrm{XF}^{\mathrm{e}} 24$ extracellular flux analyzer $(\mathrm{n}=10)$. Time points where Oligomycin (Oligo), FCCP and Antimycin/Rotenone (Ant/Rot) were injected and the rate number where each OCR was measured are indicated. B) Graph showing quantification of basal, spare, maximal, ATP-linked and non-mitochondrial respiration, and respiration due to proton leak in myotubes treated with either DMSO or $10 \mu \mathrm{M} \mathrm{CCCP} \mathrm{for} 24 \mathrm{~h}(\mathrm{n}=10)$. C) Graph showing the real-time assessment of exogenous fatty acid (palmitate) utilization in myotubes treated with either DMSO or $10 \mu \mathrm{M}$ CCCP in the absence (BSA) or presence of palmitate for $24 \mathrm{~h}(\mathrm{n}=5)$. Time points where Oligomycin (Oligo), FCCP and Antimycin/Rotenone (Ant/Rot) were injected and the rate number where each OCR was measured are indicated. D) Graph showing quantification of basal, spare, maximal, ATP-linked and non-mitochondrial respiration, and respiration due to proton leak in myotubes treated with either DMSO or $10 \mu \mathrm{M}$ CCCP in the absence (BSA) or presence of palmitate for $24 \mathrm{~h}(\mathrm{n}=5)$. E) Representative images of western blot analysis of phosphorylated (p-AMPK $\alpha$ ) and total AMPK $\alpha$ protein levels in myotubes treated with either DMSO or $10 \mu \mathrm{M}$ CCCP for $24 \mathrm{~h}$. The levels of GAPDH were assessed as a loading control $(n=3)$. F) Graph showing quantification of $\mathrm{p}-\mathrm{AMPK} \alpha$, total AMPK $\alpha$, and the ratio of p-AMPK $\alpha /$ total AMPK $\alpha$, normalized to GAPDH, in arbitrary units $(\mathrm{n}=3)$. All graphs represent mean \pm s.e.m. ${ }^{*} \mathrm{P}<0.05, * * \mathrm{P}<0.01, * * * \mathrm{P}<0.001$ (Student's ttest with the exception of the graph in (D), were performed with two-way ANOVA was performed). 


\subsection{C2C12 myotubes develop ER stress response upon CCCP treatment}

Mitochondria and Endoplasmic Reticulum (ER) directly communicate through mitochondria associated membranes (MAMs). Due to physical and functional connection, excessive reactive oxygen species (ROS) production, unfolded protein response (UPR) or failure to buffer calcium between two organelles might trigger a stress response in either or both organelles. In fact, shift of $\mathrm{Ca}^{2+}$ from ER lumen to mitochondria following mitochondrial dysfunction leads to flaws in protein folding machinery thereby resulting in the accumulation of misfolded proteins ${ }^{272}$. To determine whether CCCP-mediated mitophagy in C2C12 myotubes is associated with increased ER stress, the expression of ER stress indicators was examined. Subsequent qPCR analysis revealed upregulation of inositol requiring enzyme 1 (Irel $\alpha$ ), C/EBP homologous protein (Chop) and binding immunoglobulin protein $(B i P)$ following CCCP treatment, suggesting that CCCP induces ER stress by upregulating UPR genes (Fig. 3.4A). Activation of ER stress results in dimerization of PERK, a serine/threonine kinase, which phosphorylates and activates the $\alpha$ subunit of eukaryotic initiation factor eIF2 $\alpha$, which in turn inhibits protein synthesis and activates the ER-stress mediated apoptosis pathway through CHOP. Consistent with this, an increase in the levels of phosphorylated eIF2 $\alpha$ (p-eIF2 $\alpha$ ) and total CHOP protein were observed following CCCP treatment, but the levels of PERK remained unchanged (Fig. 3.4B \& 3.4C), suggesting that $\mathrm{CCCP}$-induced mitophagy activates the ER stress response in $\mathrm{C} 2 \mathrm{C} 12$ myotubes. Taken together, these data suggest that CCCP treatment leads to activation of PARKIN, loss of mitochondria (mitophagy) and impaired mitochondrial function leading to ER stress in $\mathrm{C} 2 \mathrm{C} 12$ myotubes. 
A

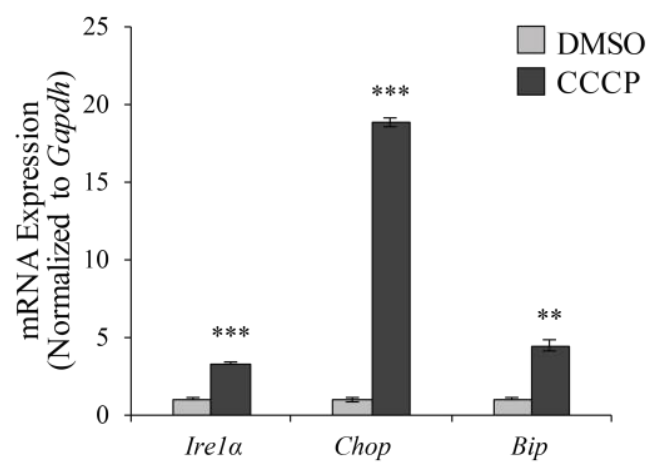

B
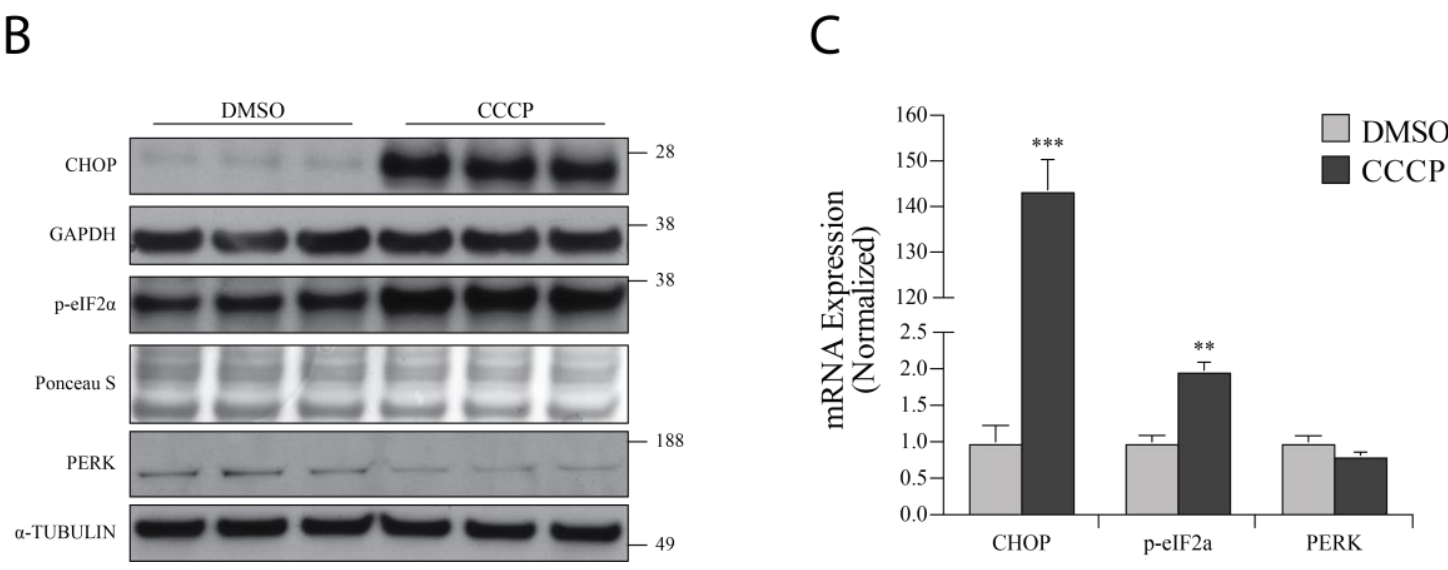

Figure 3.4: CCCP-treatment results in endoplasmic reticulum (ER) stress in C2C12 myotubes.

A) Graph showing qPCR analysis of Irel $\alpha$, Chop and Bip ER-stress marker mRNA expression, normalized to Gapdh, in $24 \mathrm{~h}$ DMSO or $10 \mu \mathrm{M}$ CCCP treated myotubes $(\mathrm{n}=3)$. B) Representative images of western blot analysis of ER-Stress marker (CHOP, p-eIF2 $\alpha$ and PERK) protein levels in myotubes treated with either DMSO or $10 \mu \mathrm{M}$ CCCP for $24 \mathrm{~h}$. The levels of GAPDH, Ponceau S or $\alpha$-TUBULIN were assessed as loading controls $(n=3)$. C) Graph showing quantification of CHOP, $p$-eIF2 $\alpha$ and PERK normalized to either GAPDH, Ponceau S or $\alpha$-TUBULIN, respectively, in arbitrary units $(\mathrm{n}=3)$. All graphs represent mean \pm s.e.m. ${ }^{* *} \mathrm{P}<0.01,{ }^{* * *} \mathrm{P}<0.001$ (Student's t-test). 


\subsection{Treatment of myotube cultures with CCCP leads to myotubular atrophy}

To explore whether CCCP-induced loss of mitochondria number and function resulted in skeletal muscle atrophy, differentiated $\mathrm{C} 2 \mathrm{C} 12$ myotubes were treated with 1 , 5 or $10 \mu \mathrm{M}$ CCCP. Treatment with $5 \mu \mathrm{M}$ and $10 \mu \mathrm{M}$ CCCP led to an observable reduction in myotube area (Fig. 3.5A). Subsequent quantification of myotube area revealed a significant reduction in myotube area upon treatment with $5 \mu \mathrm{M}$ and $10 \mu \mathrm{M}$ CCCP (Fig. 3.5B). Consistent with this, myosin heavy chain (MyHC) and myosin light chain (MyLC) protein levels were significantly decreased upon CCCP treatment (Fig. 3.5C \& 3.5D). Collectively, these data reveal that treatment with CCCP results in a notable atrophy phenotype and significant reduction in protein levels of critical myofibrillar proteins MyHC and MyLC in C2C12 myotubes.

\subsection{CCCP treatment activates Akt-FoxO pathway and expression of atrogenes}

Given that skeletal muscle atrophy is regulated by the Akt-FoxO pathway ${ }^{273,274}$, the role of Akt-FoxO pathway in CCCP-mediated muscle wasting was assessed. Surprisingly, western blot analysis revealed increased levels of phosphorylated AKT (pAKT) upon CCCP treatment (Fig. 3.6A \& 3.6B). Despite the increase in p-AKT levels, immunoblot analysis of FOXO3a transcription factor revealed a significant reduction in the levels of phosphorylated FOXO3a (p- FOXO3a), which is indicative of increased FOXO3a nuclear translocation and thereby, increased expression of atrophy-related genes (atrogenes) (Fig. 3.6C \& 3.6D). Consistent with reduced levels of p-FOXO3a, we observed a significant increase in the expression of FoxO downstream targets Atrogin1 and Murfl was observed following CCCP treatment (Fig. 3.6E). In contrast, a significant decrease in the expression of additional E3 ligases Nedd4 and Trim72 was noted following CCCP treatment (Fig. 3.6E). Taken together these data suggest that CCCP-mediated activation of the PINK1/PARKIN pathway results in skeletal muscle atrophy in $\mathrm{C} 2 \mathrm{C} 12$ myotubes, through activation of muscle-specific E3 ligases Atrogin1 and Murfl. 
A

DMSO

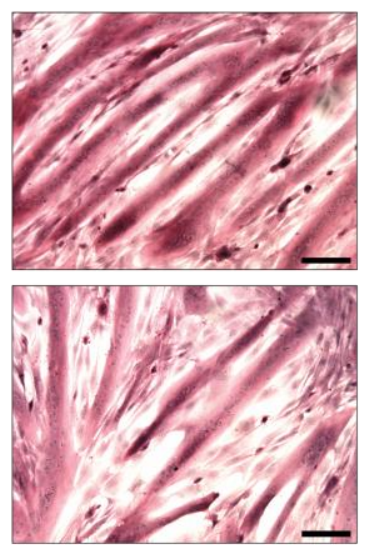

$1 \mu \mathrm{M} \mathrm{CCCP}$
DMSO
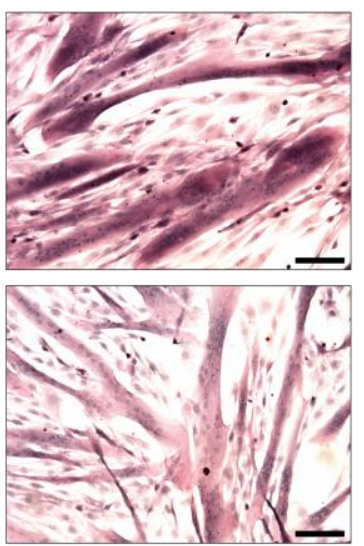

$5 \mu \mathrm{M}$ CCCP
DMSO
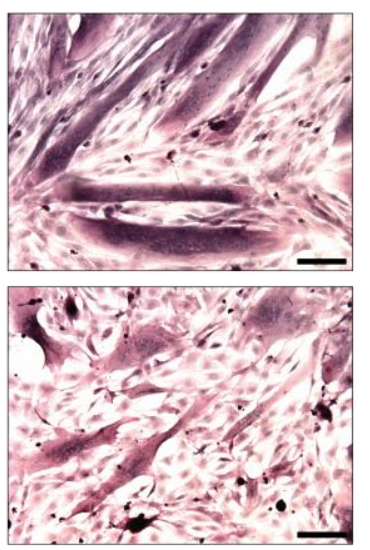

$10 \mu \mathrm{M} \mathrm{CCCP}$

B

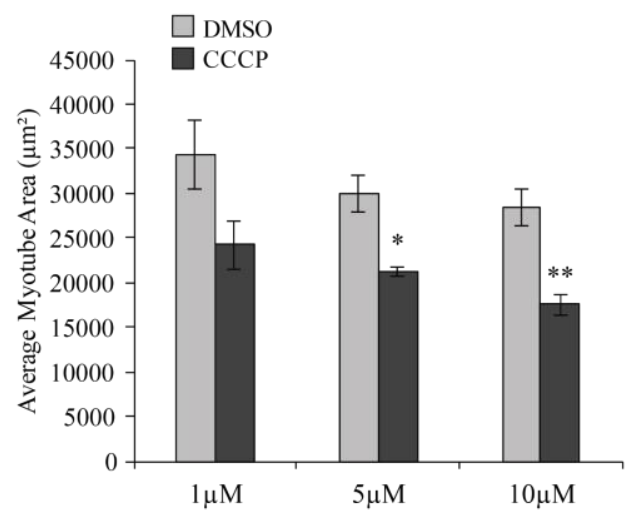

C
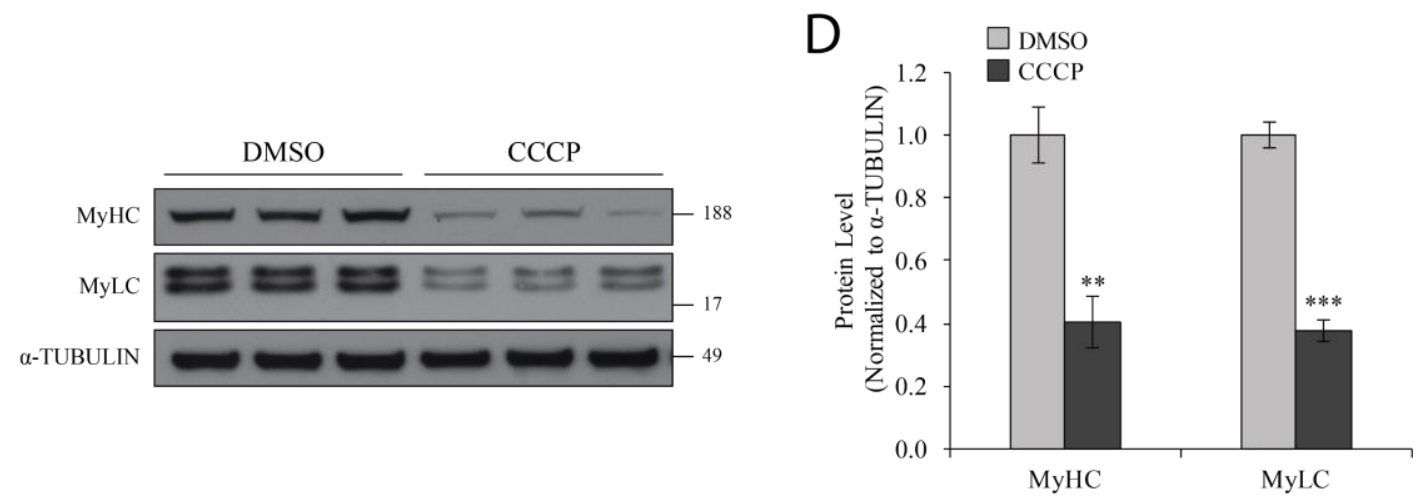

Figure 3.5: CCCP treatment leads to myotube atrophy in vitro.

A) Representative images of hematoxylin and eosin (H\&E) stained $72 \mathrm{~h}$ differentiated $\mathrm{C} 2 \mathrm{C} 12$ myotubes treated with either DMSO or increasing concentrations (1, 5 or 10 $\mu \mathrm{M})$ of CCCP for $24 \mathrm{~h}$. Scale bars represent $100 \mu \mathrm{m}$. B) Graph showing quantification of average myotube area in $72 \mathrm{~h}$ differentiated $\mathrm{C} 2 \mathrm{C} 12$ myotubes treated with either DMSO or increasing concentrations of CCCP for $24 \mathrm{~h}(\mathrm{n}=2-3)$. C) Representative images of western blot analysis of MyHC and MyLC protein levels in myotubes treated with either DMSO or $10 \mu \mathrm{M}$ CCCP for $24 \mathrm{~h}$. The levels of $\alpha$-TUBULIN were assessed as a loading control. D) Graph showing quantification of MyHC and MyLC protein levels, normalized to $\alpha$-TUBULIN, in arbitrary units $(n=3)$. All graphs display mean \pm s.e.m. ${ }^{*} \mathrm{P}<0.05,{ }^{*} * \mathrm{P}<0.01, * * * \mathrm{P}<0.001$ (Student's t-test). 
A

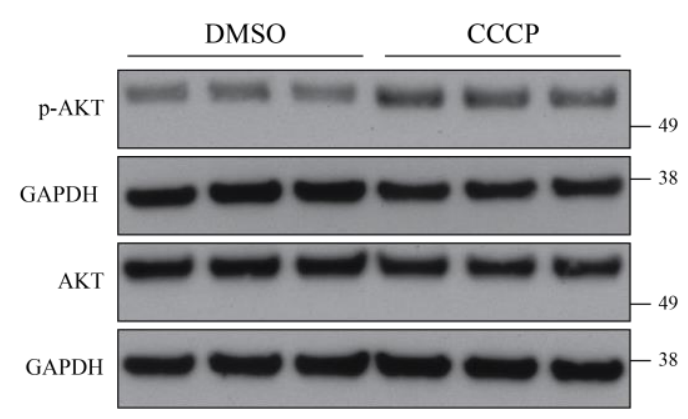

C

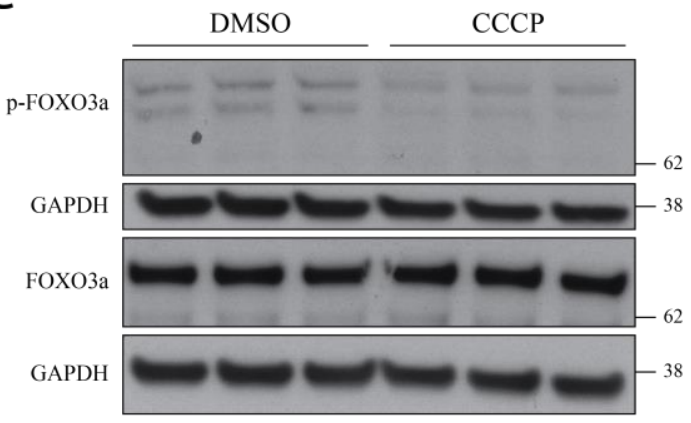

B

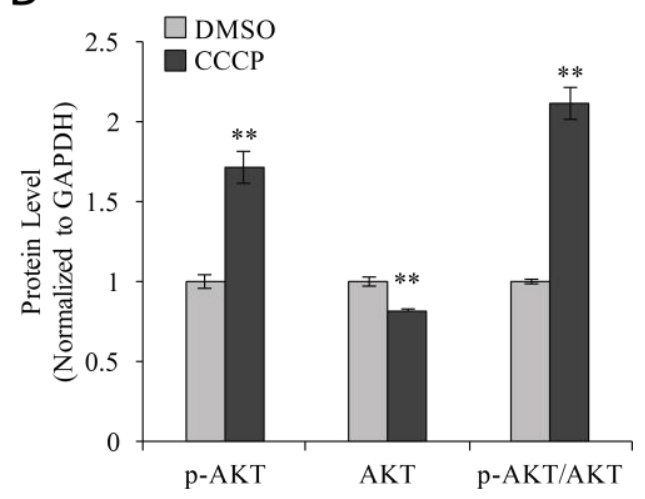

D

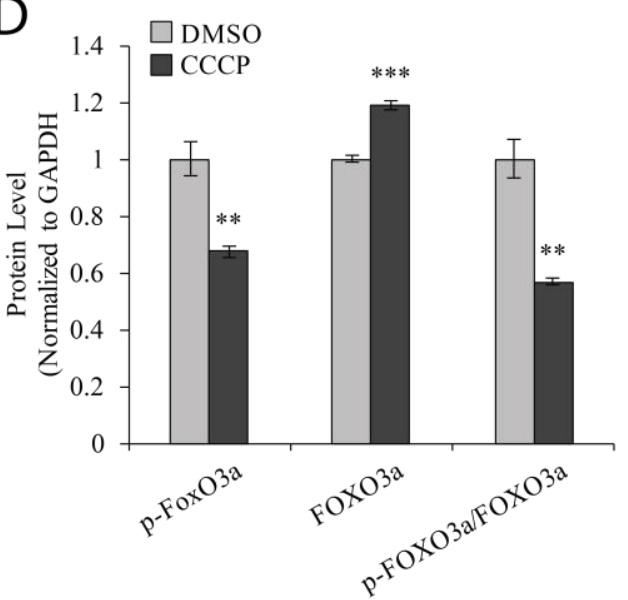

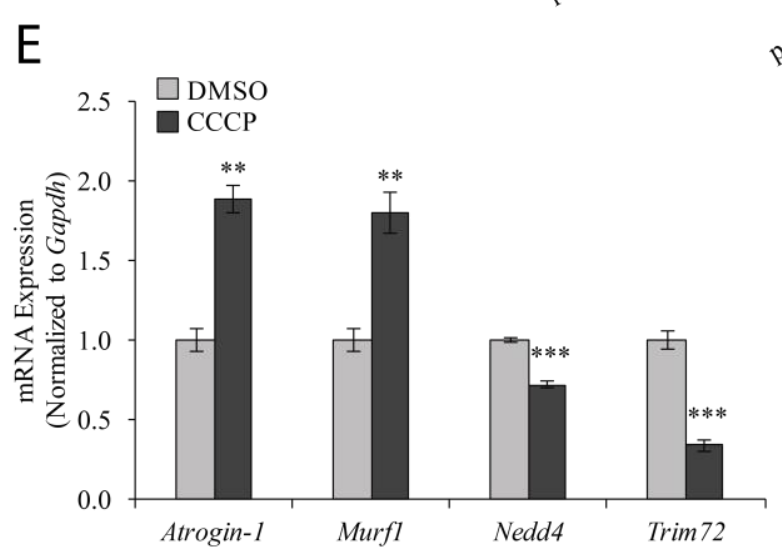

Figure 3-6: CCCP-treatment activates the transcription factor FoxO3 and enhances expression of Atrogin-1 and Murf1

A) Representative images of western blot analysis of p-AKT1/2/3 and total AKT protein levels in DMSO and $10 \mu \mathrm{M}$ CCCP treated myotubes. The levels of GAPDH were assessed as a loading control $(\mathrm{n}=3)$. B) Graph showing quantification of $\mathrm{p}-\mathrm{AKT} 1 / 2 / 3$, total AKT, and the ratio of p-AKT1/2/3/total AKT, normalized to GAPDH, in arbitrary units $(n=3)$. C) Representative images of western blot analysis of p-FOXO3a and total FOXO3a protein levels in DMSO and $10 \mu \mathrm{M}$ CCCP treated myotubes. The levels of GAPDH were assessed as a loading control $(n=3)$. D) Graph showing quantification of p-FOXO3a, total FOXO3a, and the ratio of p-FOXO3a/total FOXO3a, normalized to GAPDH, in arbitrary units (n=3). E) Graph showing qPCR analysis of Atrogin-1, Murfl, Nedd4 and Trim72 E3 ligase mRNA expression, normalized to Gapdh, in 24 h DMSO or $10 \mu \mathrm{M}$ CCCP treated myotubes $(\mathrm{n}=3)$. Data represent mean \pm s.e.m. $* * \mathrm{P}<0.01$, $* * * \mathrm{P}<0.001$ (Student's t-test). 


\subsection{Knock down of Parkin results in accumulation of dysfunctional mitochondria in myoblast cultures}

Next, the effect of Parkin knock down on mitochondrial number and function in myoblasts was assessed. qPCR analysis revealed significant knock down of Parkin in C2C12 myoblasts transfected with Parkin-specific siRNA (Fig. 3.7A). In contrast to the results observed following CCCP treatment, knock down of Parkin in myoblasts resulted in a significant increase in mtDNA copy number and MitoTracker Green FM intensity (Fig. 3.7B \& 3.7C), consistent with an increase in mitochondrial number. Despite the increase in mitochondrial number noted in response to Parkin knock down, the levels of critical OXPHOS proteins were not significantly altered between control and Parkin knock down myoblasts (Fig. 3.7D \& 3.7E), and in fact, the levels of OXPHOS proteins tended to decrease upon Parkin knock down (Fig. 3.7D \& 3.7E). In contrast, qPCR analysis revealed a significant increase in the expression of several mitochondrial complex genes in response to Parkin knock down, with increased expression of Atp6, Ndufa9, Ndufs3, Sdha, Uqcrc1, Uqcrc2 and Cox4 noted (Fig. 3.7F $\& 3.7 \mathrm{G})$. Interestingly, with the exception of Atp6, only nuclear DNA encoded mitochondrial genes were upregulated (Ndufa9, Ndufs3, Sdha, Uqcrc1, Uqcrc2 and Cox4) upon Parkin knock down in myoblasts (Fig. 3.7F \& 3.7G). Collectively, these data revealed that knock down of Parkin leads to increased mitochondrial mass and mRNA expression of several mitochondrial complex genes. 
A

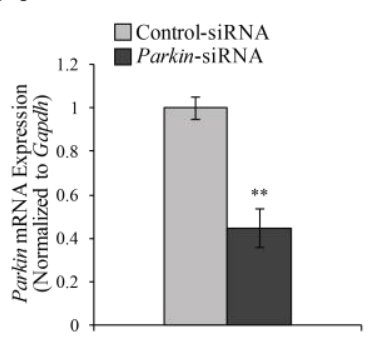

B

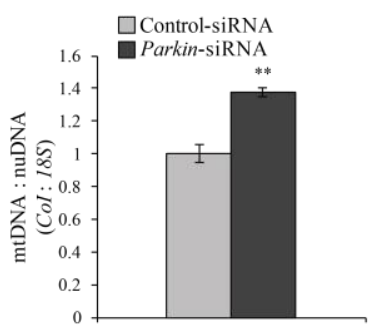

C

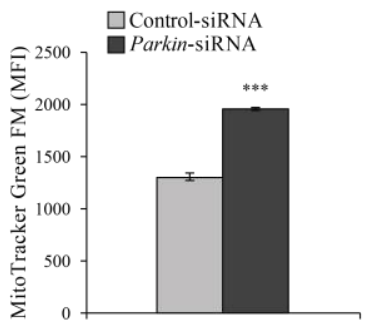

D

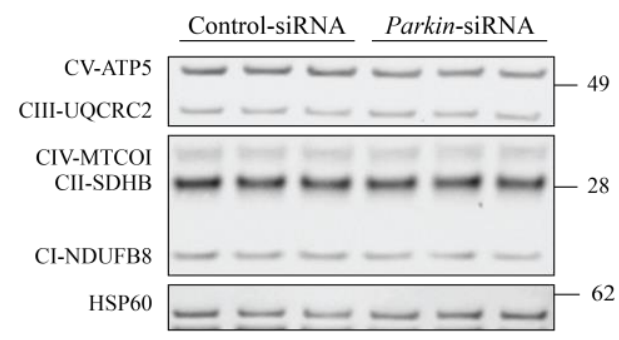

$\mathrm{F}$

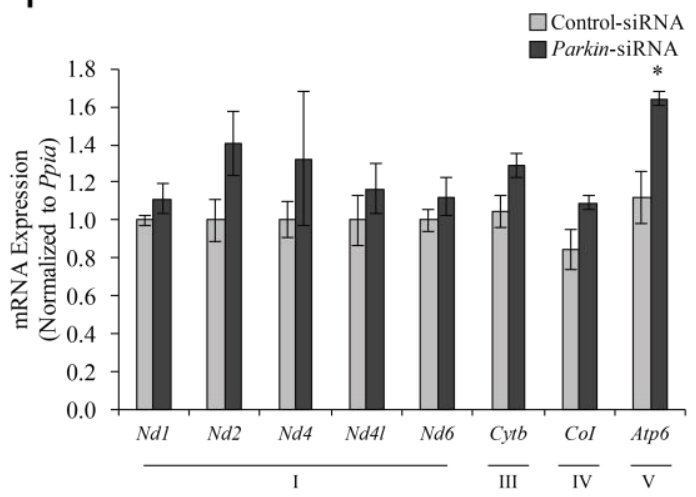

E

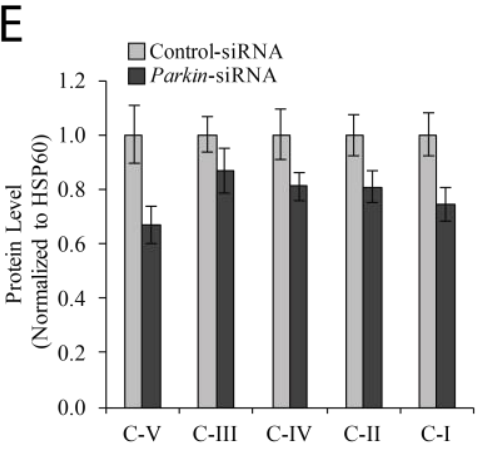

G

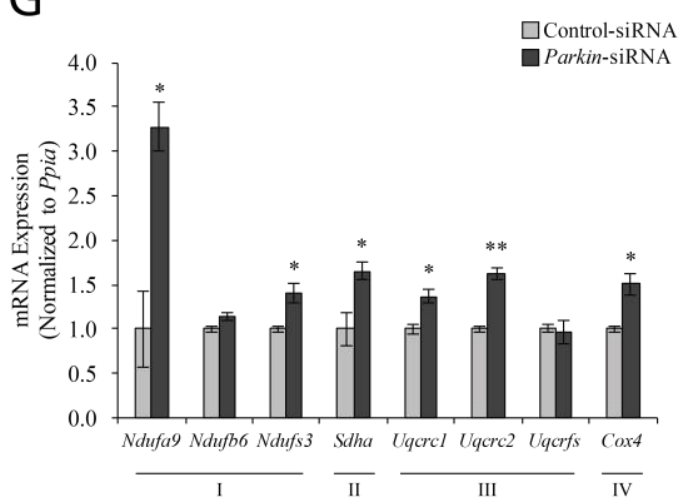

Figure 3.7: Parkin knockdown leads to accumulation of mitochondria in vitro

A) Graph showing qPCR analysis of Parkin mRNA expression, normalized to Gapdh, in Control siRNA (Control-siRNA) and Parkin-specific siRNA (Parkin-siRNA) transfected myoblasts $(n=3)$. B) Graph showing qPCR analysis of mtDNA copy number:nuDNA copy number ratio in Control-siRNA and Parkin-siRNA transfected myoblasts. The expression of the mitochondrial gene $\mathrm{CoI}$ was normalized to the nuclear gene $18 S(\mathrm{n}=3)$. C) Graph showing results of FACS analysis of MitoTracker Green FM stained Control-siRNA and Parkin-siRNA transfected myoblasts (MFI: Mean fluorescence intensity) $(\mathrm{n}=3)$. D) Representative images of western blot analysis of OXPHOS subunit (ATP5, UQCRC2, MTCOI, SDHB and NDUFB8) protein levels in Control-siRNA and Parkin-siRNA transfected myoblasts. The levels of HSP60 were assessed as a loading control $(\mathrm{n}=3)$. Two different exposures of the same membrane are provided in different boxes to visualize OXPHOS immunoreactive bands. E) Graph showing quantification of OXPHOS protein levels, normalized to HSP60, in arbitrary units $(\mathrm{n}=3)$. F) Graph showing qPCR analysis of mitochondria DNA-encoded mitochondrial complex gene expression, normalized to Ppia, in Control-siRNA and Parkin-siRNA transfected myoblasts $(\mathrm{n}=3)$. G) Graph showing $\mathrm{qPCR}$ analysis of nuclear DNA-encoded mitochondrial complex gene expression, normalized to Ppia, in Control-siRNA and Parkin-siRNA transfected myoblasts $(\mathrm{n}=3)$. All graphs display mean \pm s.e.m. ${ }^{*} \mathrm{P}<0.05,{ }^{*} * \mathrm{P}<0.01,{ }^{* * *} \mathrm{P}<0.001$ (Student's t-test). 


\subsection{Parkin knock down results in impaired mitochondrial turnover}

Given that Parkin knock down increases mitochondrial number in myoblasts, mitochondrial turnover was next analyzed. Reduced expression of mitochondrial fusion markers, Mitofusin 1 (Mfn1), Mitofusin 2 (Mfn2) and Mitochondrial dynamin-like GTPase 1 (Opal), and mitochondrial fission markers, Dynamin-1-like protein (Drpl), Mitochondrial fission 1 protein (Fisl) and Mitochondrial fission factor (Mff), was observed in Parkin knock down myoblasts, although only the expression of mitochondrial fission factor (Mff) was significantly reduced (Fig. 3.8A). Moreover, the levels of MFN1, MFN2 and DRP1 proteins were significantly reduced in Parkin knock down myoblasts (Fig. 3.8B \& 3.8C). Besides myoblasts, the protein levels of mitochondrial turnover markers were assessed in myotubes transfected with either nontargeting siRNA or Parkin-specific siRNA. A remarkable, but not significant, reduction was noted in protein levels of MFN1, whereas the levels of other mitochondrial turnover protein markers remained unchanged (Fig. 3.8D \& 3.8E). These data suggest that knock down of Parkin in myoblasts result in reduced mitochondrial fusion and fission. 


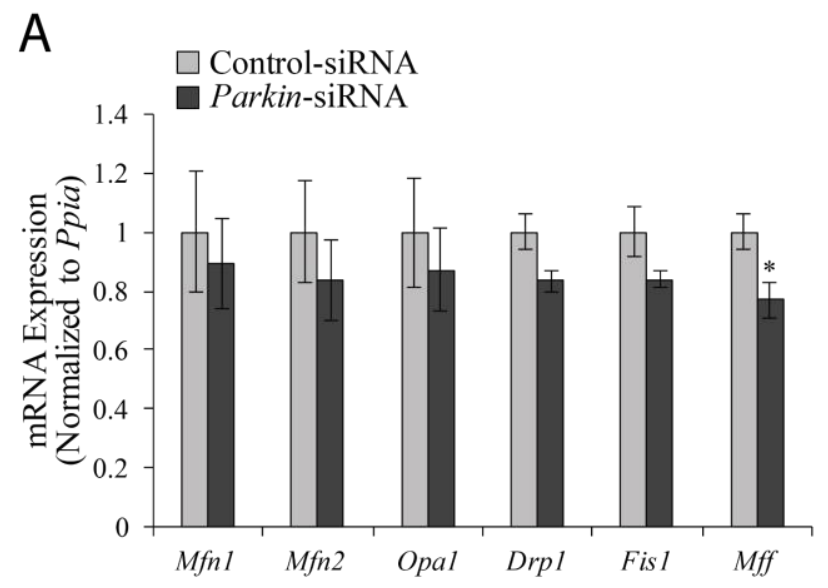

B

MYOBLASTS

Control-siRNA Parkin-siRNA

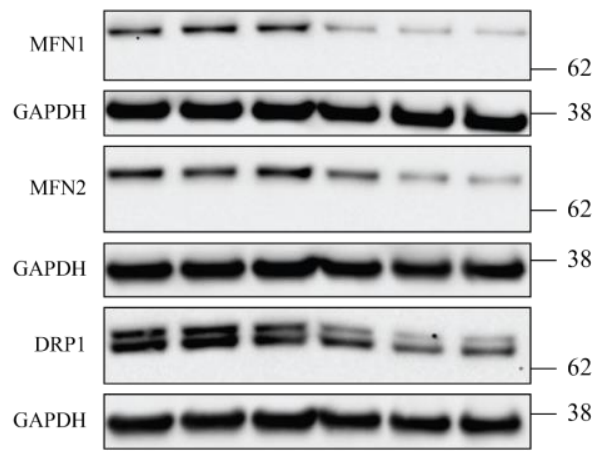

D

MYOTUBES

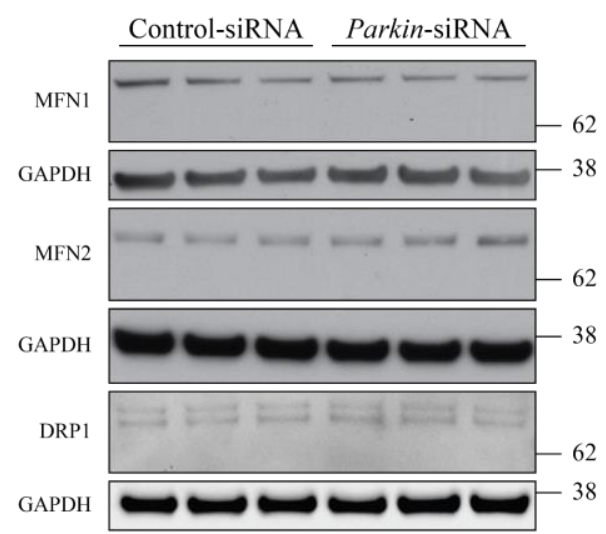

C

MYOBLASTS

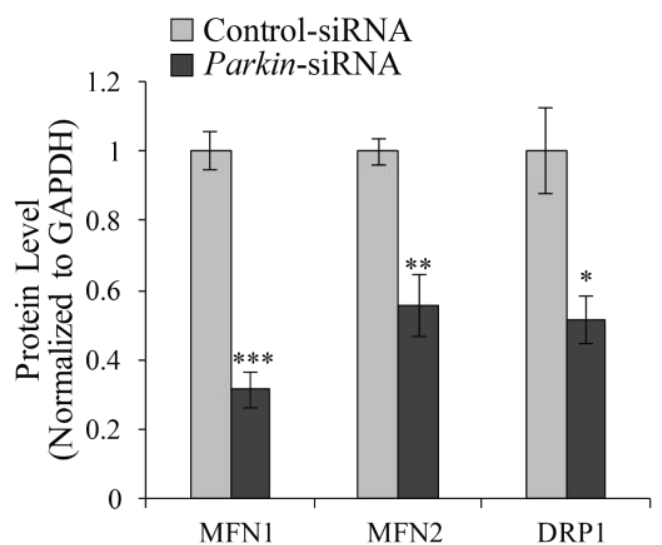

E

MYOTUBES

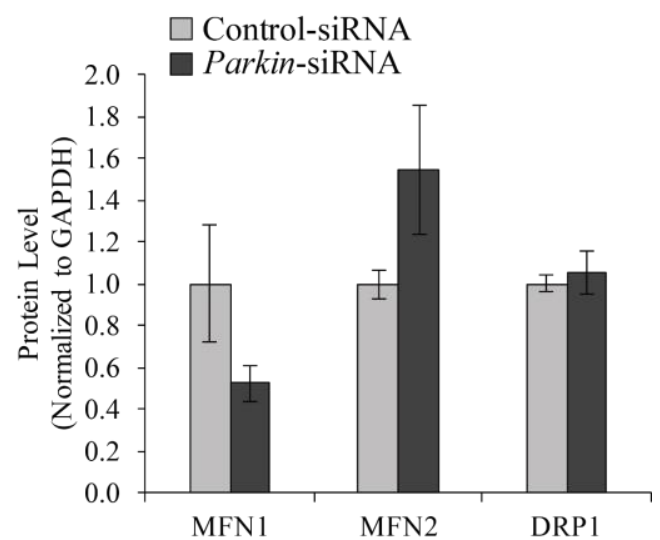


Figure 3.8: Parkin knockdown alters the levels of mitochondrial fusion and fission markers

A) Graph showing qPCR analysis of mitochondrial fusion (Mfn1, Mfn2 and Opal) and fission (Drpl, Fisl and Mff) marker gene expression, normalized to Ppia, in ControlsiRNA and Parkin-siRNA transfected myoblasts $(\mathrm{n}=3)$. B) Representative images of western blot analysis of MFN1, MFN2 and DRP1 protein levels in Control-siRNA and Parkin-siRNA transfected myoblasts. The levels of GAPDH were assessed as a loading control for each respective blot $(n=3)$. C) Graph showing quantification of MFN1, MFN2 and DRP1 protein levels, normalized to GAPDH, in arbitrary units $(n=3)$. D) Representative images of western blot analysis of MFN1, MFN2 and DRP1 protein levels in Control-siRNA and Parkin-siRNA transfected myotubes. The levels of GAPDH were assessed as a loading control for each respective blot $(n=3)$. E) Graph showing quantification of MFN1, MFN2 and DRP1 protein levels, normalized to GAPDH, in arbitrary units $(\mathrm{n}=3)$. All graphs display mean \pm s.e.m. $* \mathrm{P}<0.05, * * \mathrm{P}<0.01$, $* * * \mathrm{P}<0.001$ (Student's t-test). 


\subsection{Knock down of Parkin does not alter autophagy in vitro}

To further assess mitochondrial turnover (mitophagy), the levels of the autophagosomal markers LC3-II and P62 were analyzed. As shown in Fig. 3.9A, knockdown of Parkin reduced P62 protein levels, whereas no significant difference in endogenous LC3-II levels were noted between control and Parkin knock down myoblasts (Fig. 3.9A). Moreover, no significant difference in LC3-II levels were observed between control and Parkin-siRNA transfected myoblasts in response to Chloroquine (CQ) treatment, suggesting that autophagic flux was not significantly altered in Parkin knock down myoblasts (Fig. 3.9B). Taken together, these data suggest that the knockdown of Parkin in myoblasts results in accumulation of mitochondria, possibly due to reduced fission and thus impaired mitochondrial turnover.

\subsection{Loss of Parkin leads to reduced mitochondrial respiratory capacity, increased cellular ROS levels and activation of AMPK}

Next, mitochondrial function was assessed using the Seahorse $\mathrm{XF}^{\mathrm{e}} 24$ extracellular flux analyzer. A reduction in OCR was noted in Parkin knock down myoblasts, when compared to control (Fig. 3.10A). A significant reduction in basal and ATP-linked respiration was noted in Parkin knock down myoblasts, together with a significant reduction in OCR due to proton leak (Fig. 3.10B). Although not statistically significant, a reduction in maximum respiration and spare respiratory capacity was further noted in Parkin knock down myoblasts, when compared to controls (Fig. 3.10B). As mitochondria play an important role in detoxifying reactive oxygen species (ROS) in the cell, ROS production was measured to further assess mitochondrial function. Consistent with impaired mitochondrial function, a moderate but significant increase in the levels of ROS was noted in Parkin known down myoblasts, as measured through assessment of fluorescent $\mathrm{H}_{2}$ DCFDA (Fig. 3.10C). In addition, increased levels of active p-AMPK $\alpha$ were also observed in Parkin knock down myoblasts, when compared to controls (Fig. 3.10D \& 3.10E), further supporting impaired mitochondrial function upon knock down of Parkin. Collectively, these data reveal that Parkin knockdown in myoblasts prevents overt mitophagy and results in the accumulation of dysfunctional mitochondria in vitro. 
A
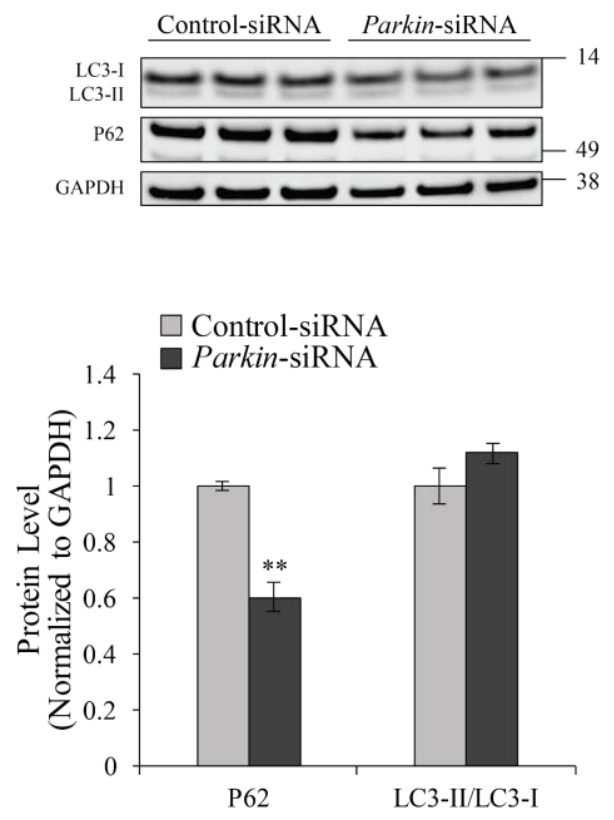

B
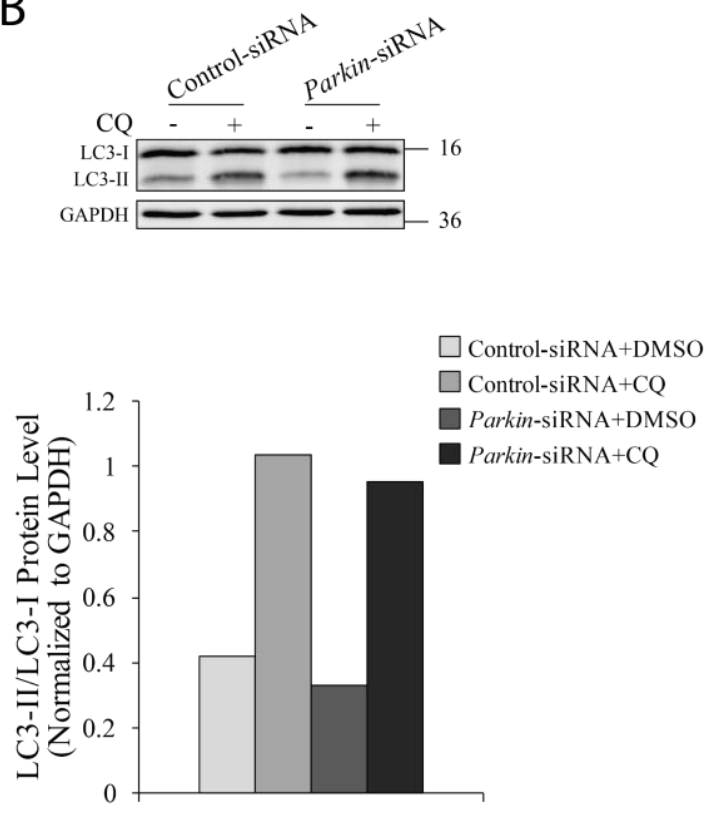

Figure 3.9: Autophagy remains unaltered upon knock down of Parkin

A) Top, Representative images of western blot analysis of P62, non-lipidated LC3 (LC3I) and lipidated LC-3 (LC3-II) protein levels in Control-siRNA and Parkin-siRNA transfected myoblasts. The levels of GAPDH were assessed as a loading control. Bottom, Graph showing quantification of P62 protein levels and the level of LC3II/LC3-I, normalized to GAPDH, in arbitrary units $(\mathrm{n}=3)$. B) Top, Representative images of western blot analysis of LC3-I and LC3-II protein levels in Control-siRNA and Parkin-siRNA transfected myoblasts treated with either DMSO (CQ; -) or chloroquine (CQ; +) for $24 \mathrm{~h}$. Each band represents results from 3 independent samples pooled together. The levels of GAPDH were assessed as a loading control. Bottom, Graph showing quantification of LC3-II/LC3-I protein levels, normalized to GAPDH, in arbitrary units. With the exception of the graph in $(\mathbf{B})$, All graphs display mean \pm s.e.m. $* * \mathrm{P}<0.01$ (Student's t-test). 
A

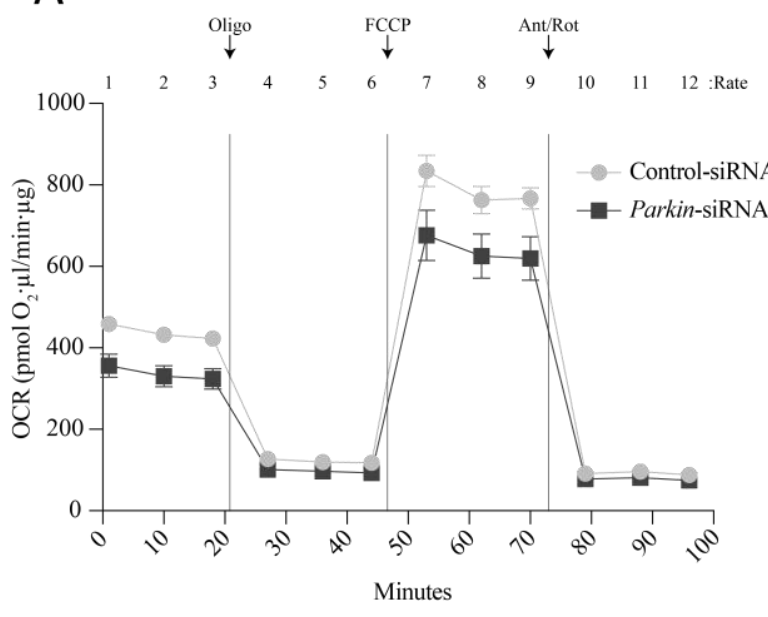

C

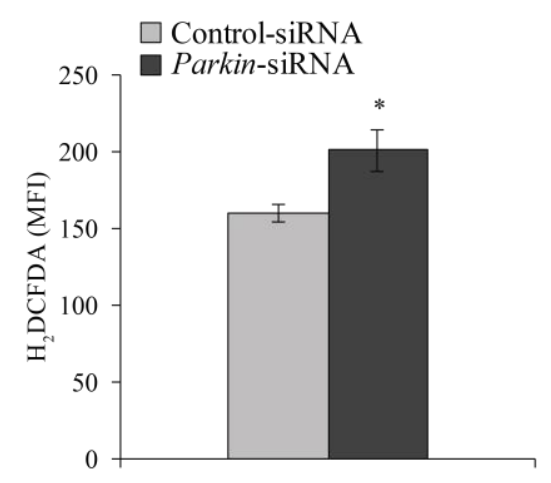

D

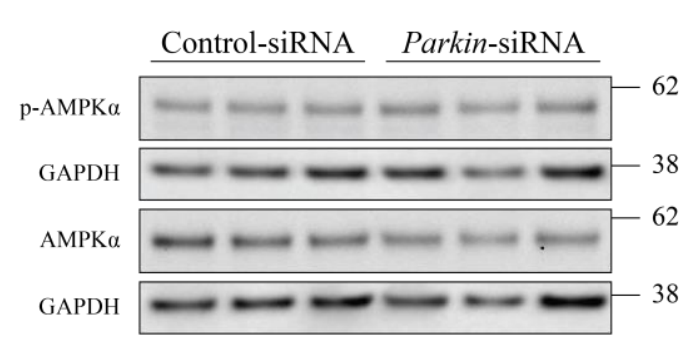

B

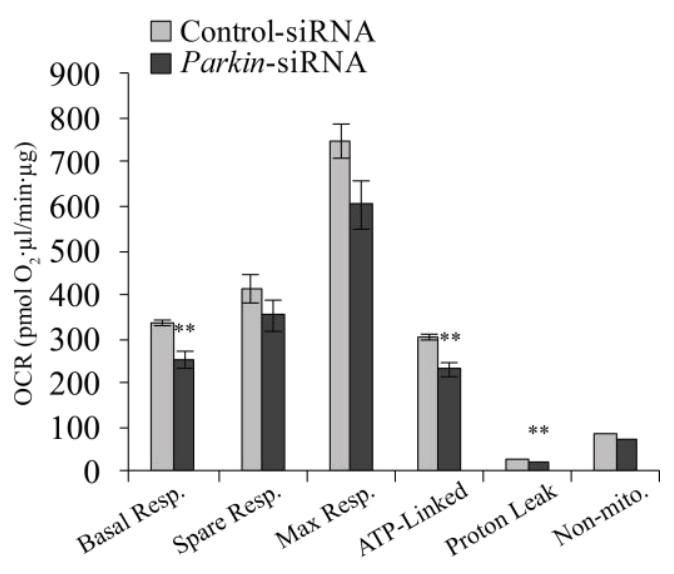

E

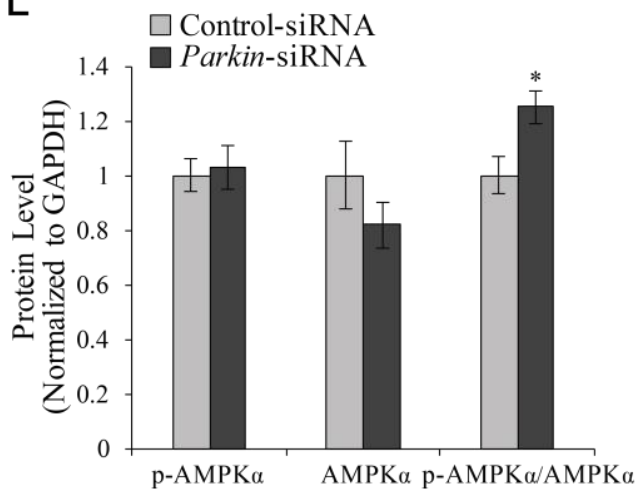


Figure 3.10: Parkin knockdown leads to mitochondrial dysfunction, increased ROS levels and AMPK $\alpha$ activation in vitro

A) Graph showing the real-time OCR of Control-siRNA and Parkin-siRNA transfected myoblasts, as assessed by the Seahorse $\mathrm{XF}^{\mathrm{2}} 24$ extracellular flux analyzer $(\mathrm{n}=5)$. Time points where Oligomycin (Oligo), FCCP and Antimycin/Rotenone (Ant/Rot) were injected (arrows) and the rate number where each OCR was measured are indicated. B) Graph showing quantification of basal, spare, maximal, ATP-linked and nonmitochondrial respiration, and respiration due to proton leak in Control-siRNA and Parkin-siRNA transfected myoblasts. All OCR values were normalized to total protein $(n=5)$. C) Graph showing the levels of Reactive Oxygen Species (ROS), as measured by $\mathrm{H}_{2}$ DCFDA fluorescence, in Control-siRNA and Parkin-siRNA transfected myoblasts $(n=4)$. D) Representative images of western blot analysis of phosphorylated AMPK $\alpha(\mathrm{p}-\mathrm{AMPK} \alpha)$ and total AMPK $\alpha$ protein levels in Control-siRNA and ParkinsiRNA transfected myoblasts. The levels of GAPDH were assessed as a loading control for each respective blot $(n=3)$. E) Graph showing quantification of $\mathrm{p}$-AMPK $\alpha$, total AMPK $\alpha$ and ratio of $\mathrm{p}$-AMPK $\alpha /$ total AMPK $\alpha$, normalized to GAPDH, in arbitrary units ( $\mathrm{n}=3) .{ }^{*} \mathrm{P}<0.05$ and ${ }^{*} * \mathrm{P}<0.01$ (Student's t-test). 


\subsection{Parkin knock down results in myotubular atrophy in vitro}

To ascertain the effect of knock down of Parkin and subsequent accumulation of dysfunctional mitochondria on myotube phenotype, $\mathrm{C} 2 \mathrm{C} 12$ myotubes were transfected with either Control or Parkin siRNA. qPCR analysis of Parkin expression confirmed knockdown of Parkin in C2C12 myotube cultures (Fig 3.11A). Subsequent histological analysis of H\&E stained myotubes revealed notable myotubular atrophy in Parkin knockdown myotubes (Fig. 3.11B). Subsequent quantification revealed a shift in myotube area distribution, with increased small $\left(<10000 \mu \mathrm{m}^{2}\right)$ and decreased large (>10000 $\mu^{2}$ ) myotubes noted (Fig. 3.11C). In addition, a significant $\sim 18 \%$ reduction in average myotube area was also observed in Parkin knock down myotubes, when compared to controls (Fig. 3.11D). These data reveal that knock down of Parkin in myoblasts not only results in accumulation of defective mitochondria, but also leads to myotubular atrophy. 

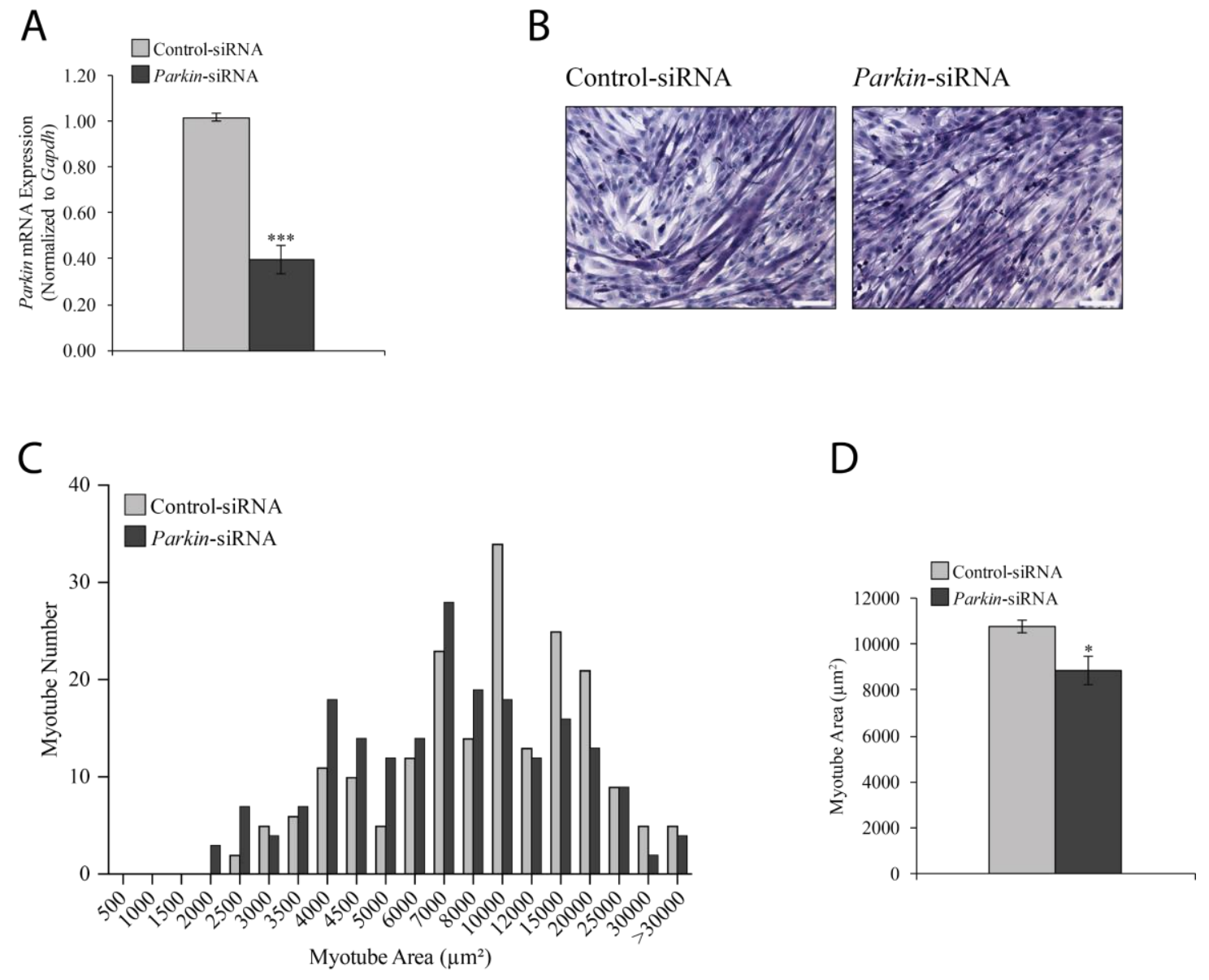

Figure 3.11: Parkin knockdown myotubes exhibit a myotube atrophy phenotype in vitro

A) Graph showing qPCR analysis of Parkin mRNA expression, normalized to Gapdh, in $120 \mathrm{~h}$ differentiated Control-siRNA and Parkin-siRNA transfected myoblasts $(\mathrm{n}=3)$. B) Representative images of H\&E stained $120 \mathrm{~h}$ differentiated Control-siRNA and Parkin-siRNA transfected myoblasts. Scale bars represent $100 \mu \mathrm{m}$. C) Graph showing the distribution of myotube area in $120 \mathrm{~h}$ differentiated Control-siRNA and ParkinsiRNA transfected myoblasts $(\mathrm{n}=4)$. D) Graph showing average myotube area in $120 \mathrm{~h}$ differentiated Control-siRNA and Parkin-siRNA transfected myoblasts $(n=4)$. With exception of the frequency distribution graph $(\mathbf{C})$, all graphs represent mean \pm s.e.m. $* \mathrm{P}<0.05$ and $* * * \mathrm{P}<0.001$ (Student's t-test). 


\subsection{Knock down of Parkin increases MURF1 protein levels and 20S proteasome activity}

Next, to determine whether atrophy phenotype observed upon Parkin knock down is resulted from activation of Akt-FoxO pathway, the levels of p-AKT were analyzed. Western blot analysis of p-AKT and total AKT and subsequent quantification of protein levels revealed a significant increase in p-AKT and AKT levels, although p-AKT/AKT ratio remained unaltered, upon Parkin knock down in myotubes (Fig 3.12A \& 3.12B). No significant difference in the protein levels or phosphorylation status of either FOXO1 or FOXO3a was noted in Parkin knock down myotubes (Fig. 3.12C \& 3.12D \& 3.12E \& 3.12F). Loss of Parkin did not alter the gene expression of Atrogin-1 and Murfl (Fig. 3.13A). However, subsequent western blot analysis revealed a moderate, but significant, increase in MURF1 protein levels, but not ATROGIN-1, upon knock down of Parkin (Fig. 3.13B \& 3.13C). Hypercatabolism in skeletal muscle is commonly observed in response to muscle wasting stimuli and importantly, the ubiquitin proteasome pathway plays a critical role in degradation of myofibrillar proteins in skeletal muscle. To assess the activity of the proteasomal system in response to knock down of Parkin, C2C12 myotubes were transfected with either control or Parkin-siRNA for $24 \mathrm{~h}$. Subsequent assessment of trypsin-like, chymotrypsin-like and caspase-like activity of the 20S proteasome's catalytic core using luminescence-based assay revealed increased chymotrypsin-like and caspase-like activity in Parkin knock down myotubes, whereas trypsin-like activity remained unaltered (Fig. 3.13D). Collectively, these data suggest that knock down of Parkin leads to myotubullar atrophy, presumably through activation of the proteasomal system and to some extend increased MURF1.

\subsection{Protein synthesis remains unaltered upon knock down of Parkin}

Besides proteasome activity, the extend of protein synthesis in Parkin-siRNA transfected $\mathrm{C} 2 \mathrm{C} 12$ myotubes was also assessed, as compared to controls. Previous studies have reported that mechanical loading, growth factors or availability of adequate nutrition modulate protein synthesis in skeletal muscle, mainly through regulation of translation initiation, which is regulated by $\mathrm{mTOR}^{275,276}$. Western blot analysis and subsequent quantification of protein levels revealed no significant difference in phosphorylated mTOR (p-mTOR), total mTOR and p-mTOR/ total mTOR ratio between Parkin knock down and control myotubes (Fig. 3.14A \& 3.14B). Also, western blot analysis revealed that protein levels of phosphorylated P70S6K (p-P70S6K) and 
total P70S6K were comparable between Parkin knock down myotubes and control cells further suggesting that protein synthesis was not affected upon knock down of Parkin (Fig. 3.14C \& 3.14D). To detect nascent protein synthesis, cells were incubated in methionine-free medium, supplemented with amino acid analog of methionine, homopropargylglycine (HPG). Results revealed that the levels of nascent protein synthesis were comparable between Control-siRNA and Parkin-siRNA transfected myotubes (Fig. 3.14E). All together, these data suggest that knockdown of Parkin results in increased proteasome activity, whereas protein synthesis is not altered in vitro. 
A

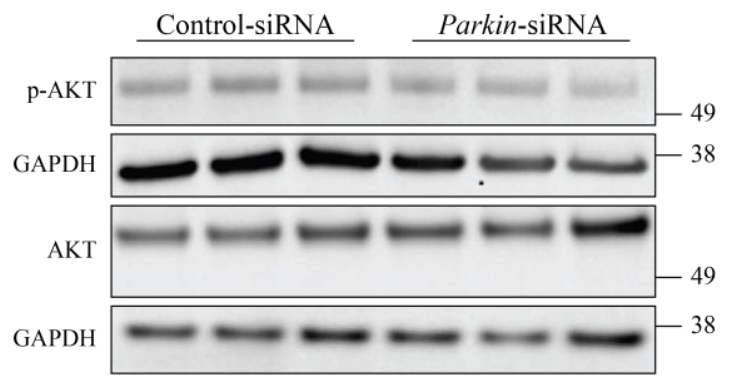

C

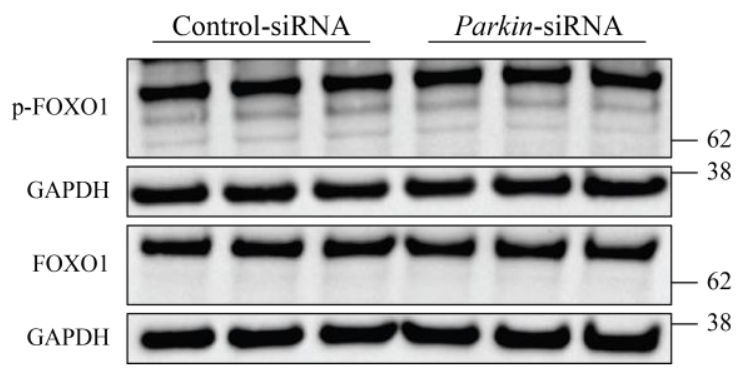

E

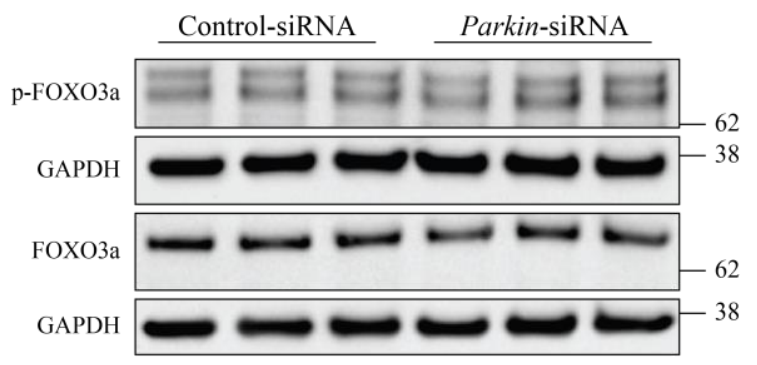

B

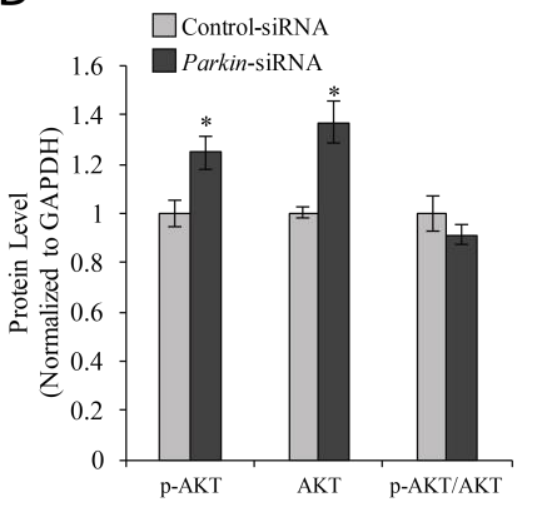

D

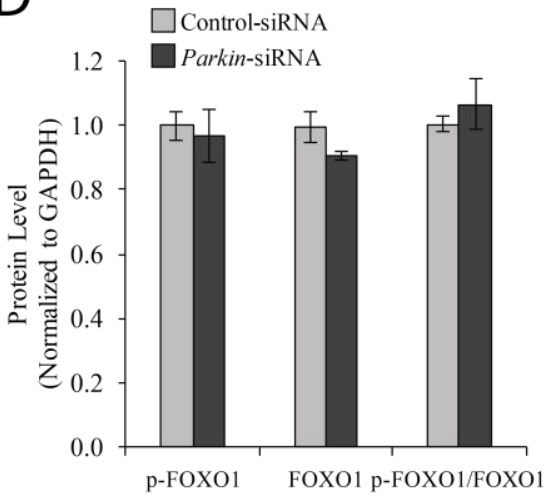

F

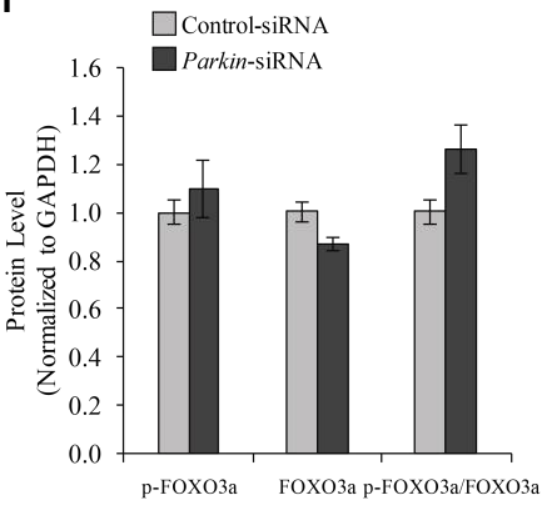


Figure 3.12: Loss of Parkin does not lead to activation of FoxO transcription factors A) Representative images of western blot analysis of p-AKT1/2/3 and total AKT protein levels in Control-siRNA and Parkin-siRNA transfected myotubes. The levels of GAPDH were assessed as a loading control $(n=3)$. B) Graph showing quantification of p-AKT1/2/3, total AKT, and the ratio of p-AKT1/2/3/total AKT, normalized to GAPDH, in arbitrary units $(n=3)$. C) Representative images of western blot analysis of p-FOXO1 and total FOXO1 protein levels in Control-siRNA and Parkin-siRNA transfected myotubes. The levels of GAPDH were assessed as a loading control $(n=3)$. D) Graph showing quantification of p-FOXO1, total FOXO1, and the ratio of pFOXO1/total FOXO1 levels, normalized to GAPDH, in arbitrary units $(n=3) \mathbf{E})$ Representative images of western blot analysis of p-FOXO3a and total FOXO3a protein levels in Control-siRNA and Parkin-siRNA transfected myotubes. The levels of GAPDH were assessed as a loading control $(n=3)$. F) Graph showing quantification of p-FOXO3a, total FOXO3a, and the ratio of p-FOXO3a/total FOXO3a levels, normalized to GAPDH, in arbitrary units $(n=3)$. Data represent mean \pm s.e.m. with error bars indicating s.e.m. $* \mathrm{P}<0.05$ (Student's t-test). 


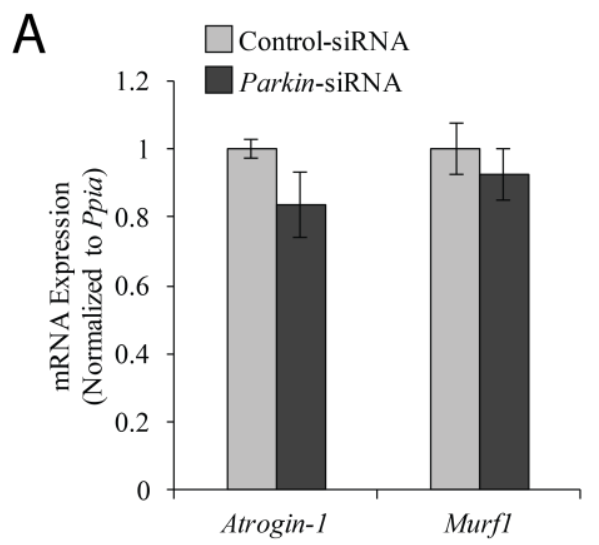

B

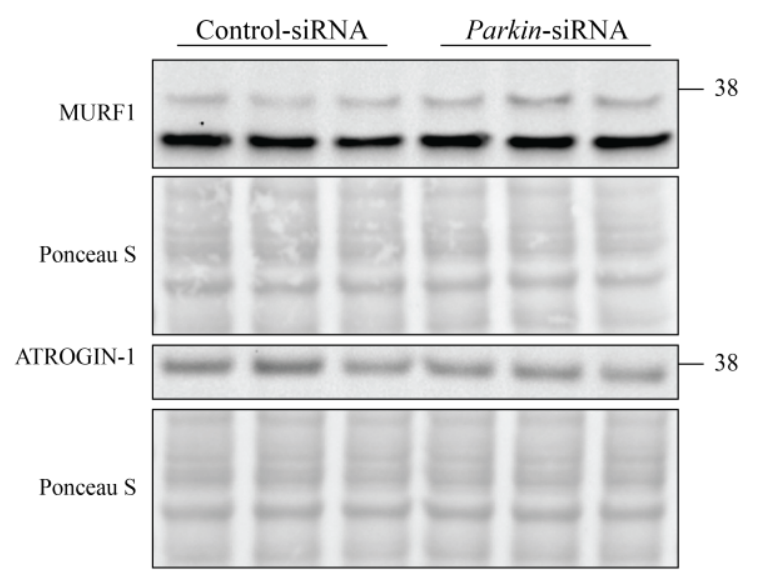

D

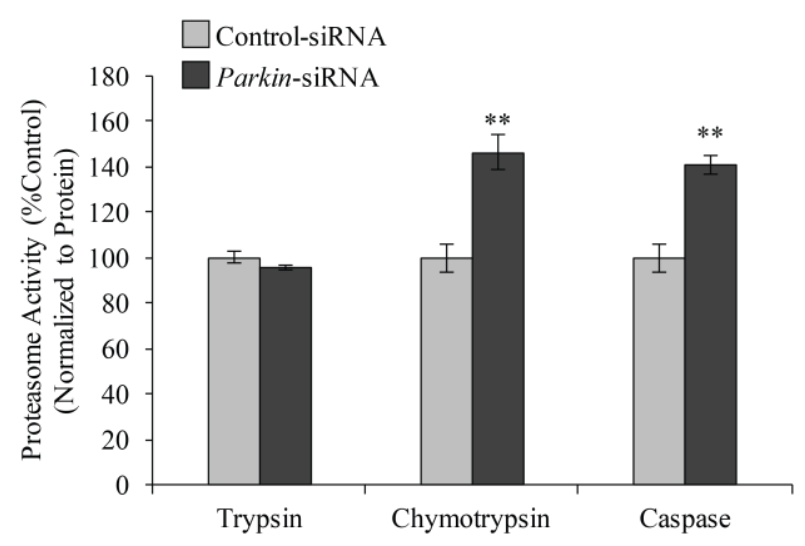

C

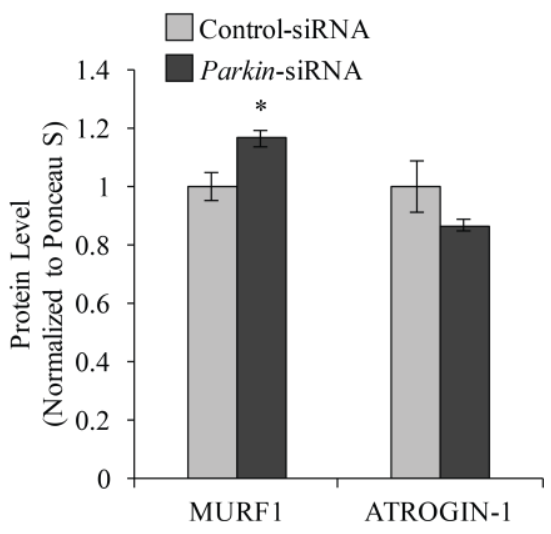


Figure 3.13: Knock down of Parkin increases MURF1 protein levels and activity of the $20 S$ proteaseome

A) Graph showing qPCR analysis of Atrogin-1 and Murfl mRNA expression in ControlsiRNA and Parkin-siRNA transfected myotubes $(n=3)$. B) Representative images of western blot analysis of MURF1 and ATROGIN-1 protein levels in Control-siRNA and Parkin-siRNA transfected myotubes. The levels of Ponceau $S$ were assessed as a loading control ( $\mathrm{n}=3)$. C) Graph showing quantification of MURF1 and ATROGIN-1 protein levels, normalized to Ponceau $S$, in arbitrary units $(n=3)$. D) Graph showing the levels of trypsin-like, chymotrypsin-like and caspase-like proteasomal activity of the $20 \mathrm{~S}$ proteasome in Control-siRNA and Parkin-siRNA transfected myotubes. Relative light units (RLU) were normalized to total protein and the graph represents percentage as compared to the control $(\mathrm{n}=4)$. Data represent mean \pm s.e.m. $* \mathrm{P}<0.05, * * \mathrm{P}<0.01$ (Student's t-test). 
A

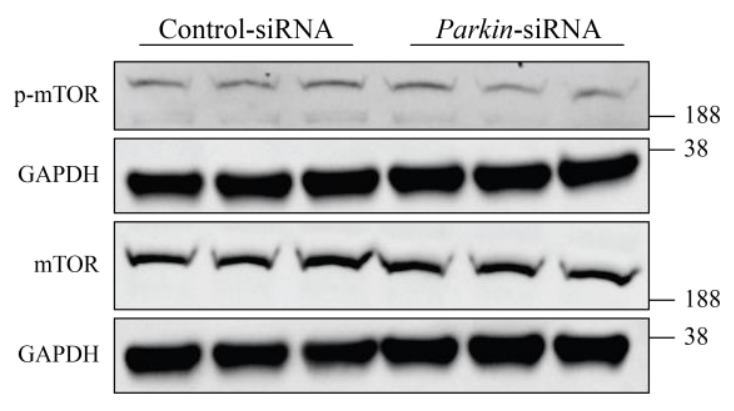

C

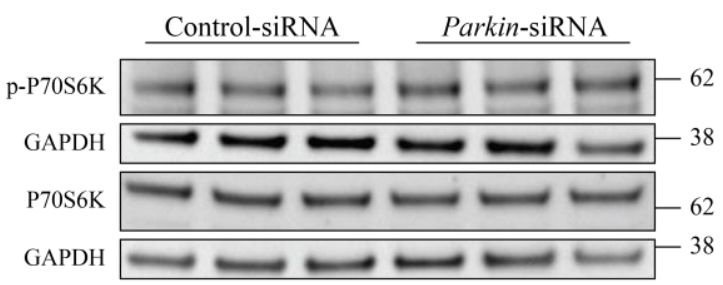

$E$

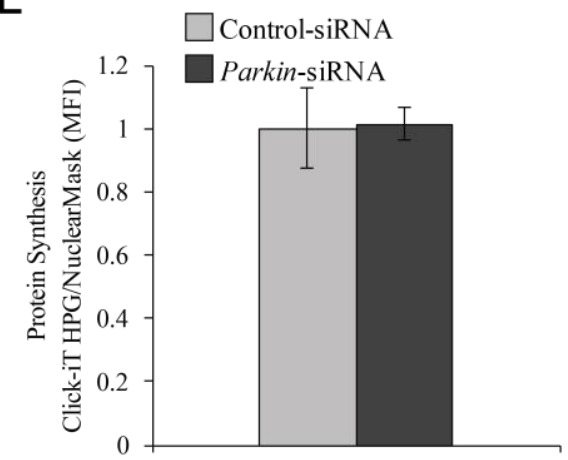

B

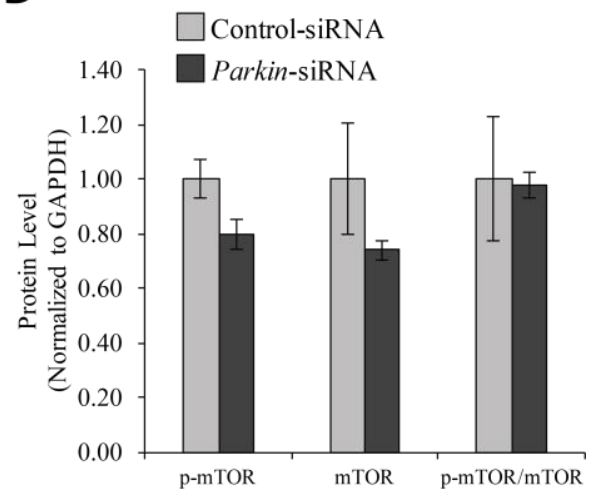

$\mathrm{D}$

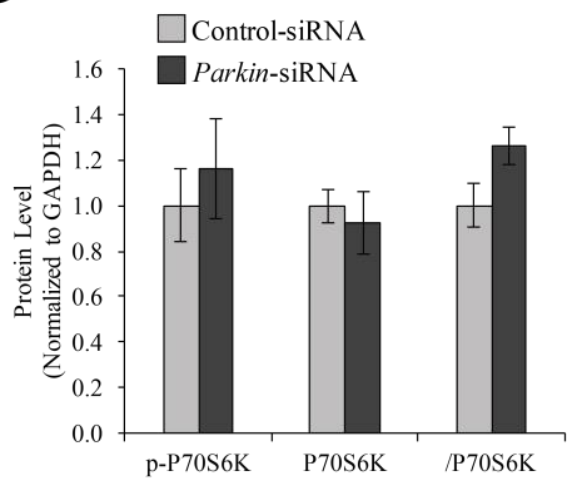

Figure 3.14: Parkin knock down does not alter protein synthesis

A) Representative images of western blot analysis of phosphorylated mTOR (p-mTOR) and total mTOR in Control-siRNA and Parkin-siRNA transfected myotubes. The levels of GAPDH were assessed as a loading control. B) Graph showing quantification of protein levels of p-mTOR and total mTOR and the ratio of p-mTOR/ total mTOR, normalized to GAPDH, in arbitrary units $(\mathrm{n}=3)$. C) Representative images of western blot analysis of phosphorylated P70S6K (p-P70S6K) and total P70S6K in ControlsiRNA and Parkin-siRNA transfected myotubes. The levels of GAPDH were assessed as a loading control. D) Graph showing quantification of protein levels of p-P70S6K and total P70S6K and ratio of p-P70S6K / total P70S6K, normalized to GAPDH, in arbitrary units (n=3). E) Graph showing nascent protein synthesis in Control-siRNA and Parkin-siRNA transfected myotubes. Mean fluorescence intensity (MFI) of Click-iT HPG Alexa Fluor 488 was normalized to NuclearMask Blue Stain fluorescence intensity $(\mathrm{n}=8)$. Data represent mean \pm s.e.m. (Student's t-test). 


\subsection{Mitochondrial number is preserved and mitochondrial OXPHOS gene expression is reduced in Parkin KO primary myotube cultures}

Given that knockdown of Parkin in vitro results in impaired mitochondrial function, mitochondrial number and function in skeletal muscle and primary myoblast cultures derived from Parkin KO mice were assessed. Although a trend towards increased mitochondrial DNA (mtDNA) copy number was noted in Parkin KO primary myoblast cultures (Fig. 3.15A), the increase was not statistically significant. Similarly, no significant difference in mitochondrial number, as assessed through MitoTracker Green FM staining and FACS analysis, was noted in Parkin KO primary myoblast cultures (Fig. 3.15B). Also, mRNA expression of mitochondrial DNA-encoded and nuclear DNA-encoded mitochondrial genes was determined through qPCR analysis. Results revealed a significant increase in the expression of the mitochondrial DNA-encoded gene Atp6 in Parkin KO myotube cultures (Fig. 3.15C). A significant reduction in the mRNA expression of the nuclear-DNA encoded mitochondrial genes $N d u f a 9, N d u f s 3$, Sdha, Uqcrc2, Uqcrfs and CoxIV was also noted (Fig. 3.15D), suggesting that OXPHOS activity is impaired in Parkin KO myotubes.

\subsection{Parkin KO primary myotubes have impaired mitochondrial turnover}

Next, to determine the effect of Parkin knock out on mitochondrial turnover, mRNA expression and protein levels of mitochondrial fission and fusion markers were assessed. The expression of mitochondrial fusion genes, $M f n l$ and $O p a l$, and the mitochondrial fission gene Drpl were significantly reduced in Parkin KO primary myotubes, when compared to wild type controls (Fig. 3.15E). A reduction in the expression of $M f n 2$ was also noted in Parkin KO primary myotubes, however the reduction was not statistically significant (Fig. 3.15E). Subsequent western blot analysis revealed a significant reduction in the levels of the mitochondrial fusion marker MFN1 in Parkin KO myotubes (Fig. 3.15F \& 3.15G). These data suggest that knock out of Parkin is associated with maintenance of mitochondrial number, impaired mitochondrial turnover and reduced expression of mitochondrial OXPHOS genes in primary myoblast cultures. 
A

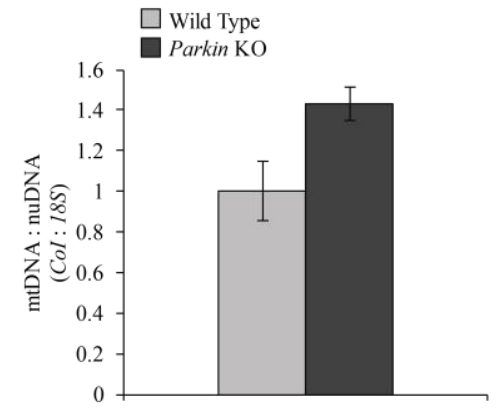

C

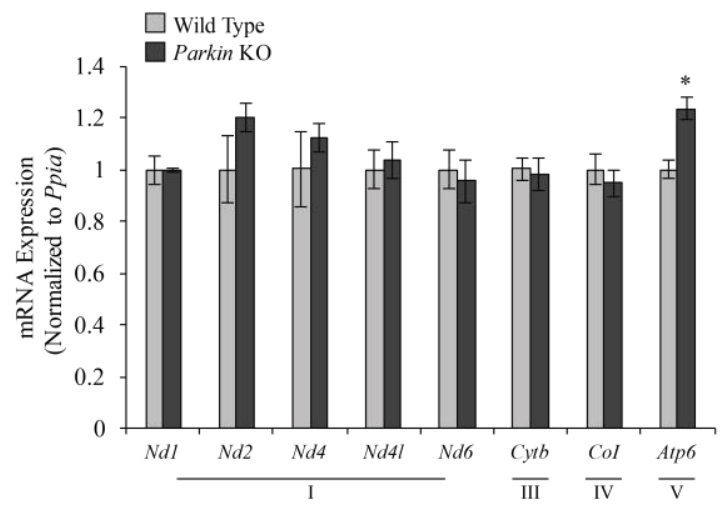

E

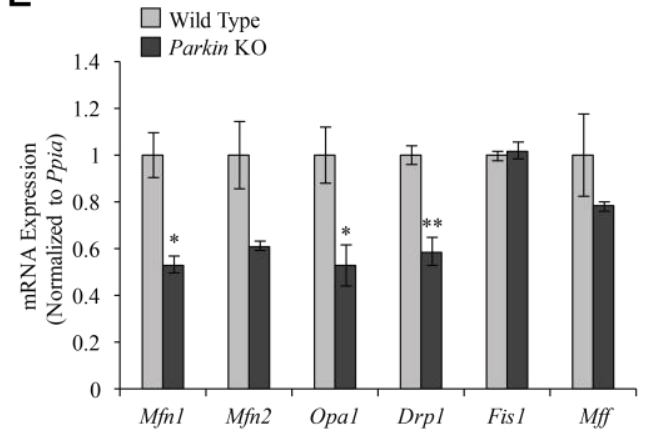

F

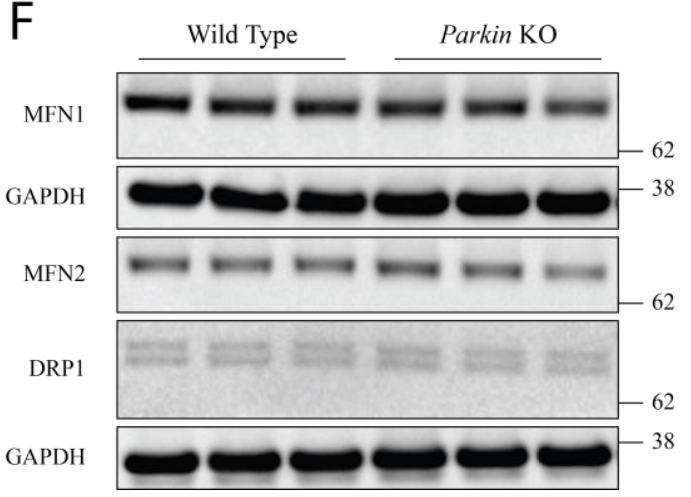

B

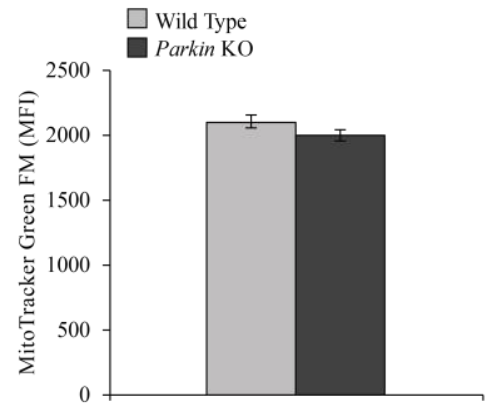

D

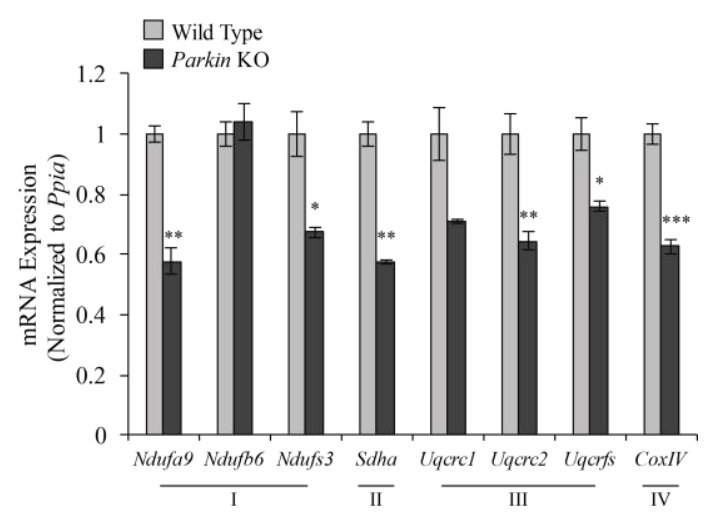

G

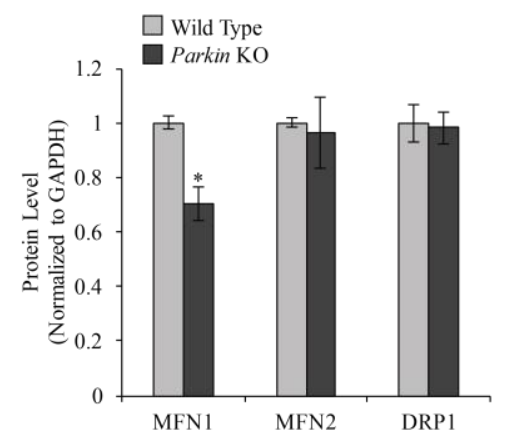


Figure 3.15: Knock out of Parkin results in reduced OXPHOS gene expression, impaired mitochondrial turnover and preservation of the mitochondrial pool in primary myotube cultures

A) Graph showing qPCR analysis of mtDNA copy number:nuDNA copy number ratio in primary myoblasts isolated from wild type and Parkin $\mathrm{KO}$ mice. Values are expressed as fold change relative to wild type control. The expression of the mitochondrial gene CoI was normalized to the nuclear gene $18 S(\mathrm{n}=3)$. B) Graph showing results of FACS analysis of MitoTracker Green FM stained primary myoblasts isolated from wild type or Parkin KO mice (MFI: Mean fluorescence intensity) $(\mathrm{n}=3)$. C) Graph showing qPCR analysis of mitochondria DNA-encoded mitochondrial complex gene expression, normalized to Ppia, in wild type and Parkin KO primary myotubes $(\mathrm{n}=3)$. D) Graph showing qPCR analysis of nuclear DNA-encoded mitochondrial complex gene expression, normalized to Ppia, in wild type and Parkin KO primary myotubes $(\mathrm{n}=3)$. E) Graph showing qPCR analysis of mitochondrial fusion (Mfn1, Mfn2, Opal) and mitochondrial fission (Drpl, Fisl, Mff) marker mRNA expression in wild type and Parkin KO primary myotubes $(\mathrm{n}=3)$. F) Representative images of western Blot analysis of MFN1, MFN2, FIS1 and DRP1 in wild type and Parkin KO primary myotubes. The levels of GAPDH were assessed as a loading control. G) Graph showing quantification of MFN1, MFN2 and DRP1 protein levels, normalized to GAPDH, in arbitrary units $(\mathrm{n}=3) . * \mathrm{P}<0.05, * * \mathrm{P}<0.001, * * * \mathrm{P}<0.0001$ (Student's t-test). 


\subsection{Reduced expression and protein levels of critical OXPHOS genes is observed in Parkin knock out Gas muscle}

Despite no significant difference in mitochondrial number, a trend towards reduced levels of mitochondrial OXPHOS proteins (Complex I-V) was observed in Parkin KO Gas muscle, when compared to wild type controls (Fig. 3.16A \& 3.16B). Notably, a significant reduction in the levels of the complex I protein NDUFB8 and the complex II protein SDHB was observed (Fig. 3.16A \& 3.16B). Consistent with this, decreased expression of both mitochondrial-encoded (Fig. 3.16C) and nuclear-encoded (Fig. 3.16D) mitochondrial complex genes was also observed in Gas muscle isolated from Parkin KO mice, with a significant reduction in Nd1, Nd6, Atp6 and Ndufb6 expression noted (Fig. 3.16C \& 3.16D).

\subsection{Knock out of Parkin leads to impaired mitochondrial turnover but not autophagy in muscle}

Next, to quantify the expression of genes critical for mitochondrial fusion ( $M f n l$, $M f n 2$ and $O p a 1$ ) and mitochondrial fission (Drpl, Fis1 and Mff) in skeletal muscle from wild type and Parkin KO mice, qPCR analysis was performed. As shown in Fig. 3.17A, no significant difference in the expression of mitochondrial fusion and fission markers was observed in Gas muscle isolated from wild type and Parkin KO mice (Fig. 3.17A). Subsequent immunoblot analysis revealed no significant difference in the levels of both MFN1 and MFN2 mitochondrial fusion proteins in Gas muscle isolated from wild type and Parkin KO mice (Fig. 3.17B \& Fig. 3.17C), which is in agreement with the qPCR results. However, a significant reduction in the protein levels of the mitochondrial fission markers FIS1 and DRP1 was noted in Parkin KO Gas muscle tissue, when compared to wild type controls (Fig. 3.17B \& Fig. 3.17C). In addition, the levels of the autophagosomal markers LC3-II and P62 were not significantly altered between muscle tissue isolated from wild type and Parkin KO mice (Fig. 3.17D \& Fig. 3.17E), suggesting that loss of Parkin does not result in overt mitophagy in skeletal muscle. These data suggest that mitochondria number is preserved in Parkin KO mice and that loss of Parkin leads to changes in gene expression consistent with decreased mitochondrial fission in skeletal muscle (Fig. 3.17D \& Fig. 3.17E). 
A

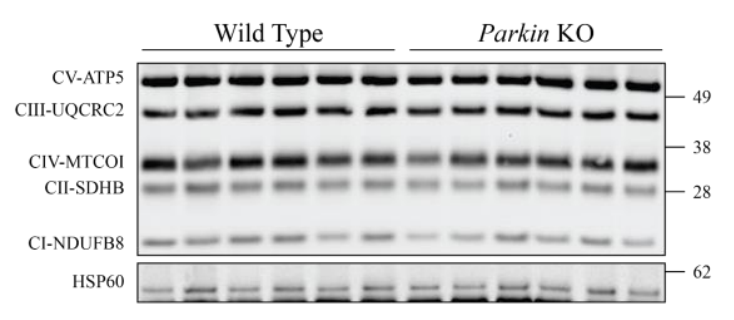

C

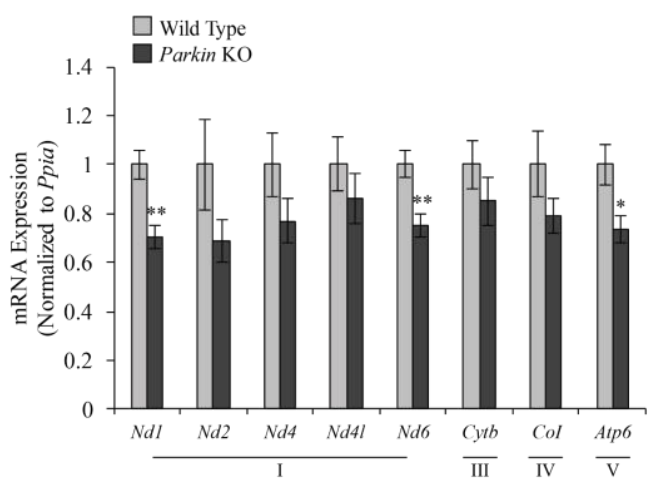

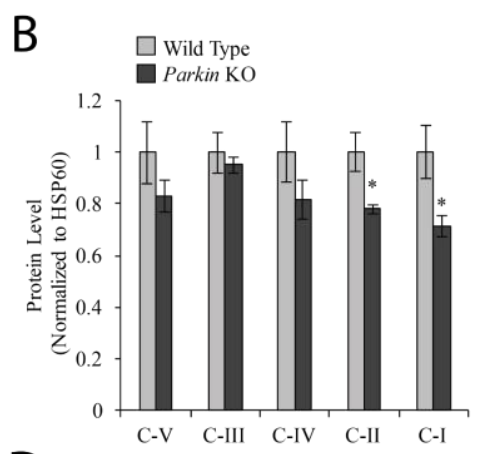

D

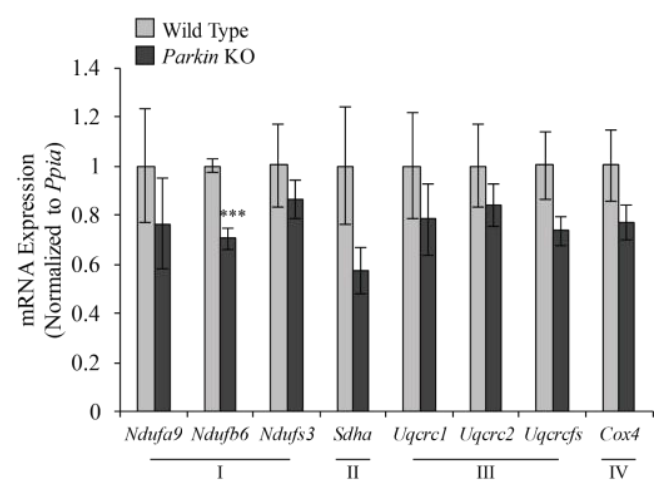

Figure 3.16: Parkin KO mice have reduced levels of mitochondrial Complex I and Complex II proteins and reduced expression of OXPHOS genes in Gas muscle

A) Representative images of western blot analysis of OXPHOS subunit (ATP5, UQCRC2, MTCOI, SDHB and NDUFB8) protein levels in wild type and Parkin KO Gas muscle. The levels of HSP60 was assessed as a loading control $(n=6)$. B) Graph showing quantification of OXPHOS protein levels, normalized to HSP60, in arbitrary units $(\mathrm{n}=6)$. C) Graph showing qPCR analysis of mitochondria DNA-encoded mitochondrial complex gene expression, normalized to Ppia, in wild type and Parkin $\mathrm{KO}$ Gas muscle $(\mathrm{n}=6)$. D) Graph showing qPCR analysis of nuclear DNA-encoded mitochondrial complex gene expression, normalized to Ppia, in wild type and Parkin KO Gas muscle ( $\mathrm{n}=6$ ). All graphs show mean \pm s.e.m. ${ }^{*} \mathrm{P}<0.05,{ }^{*} * \mathrm{P}<0.01$, ${ }^{*} * \mathrm{P}<0.001$ (Student's t-test). 


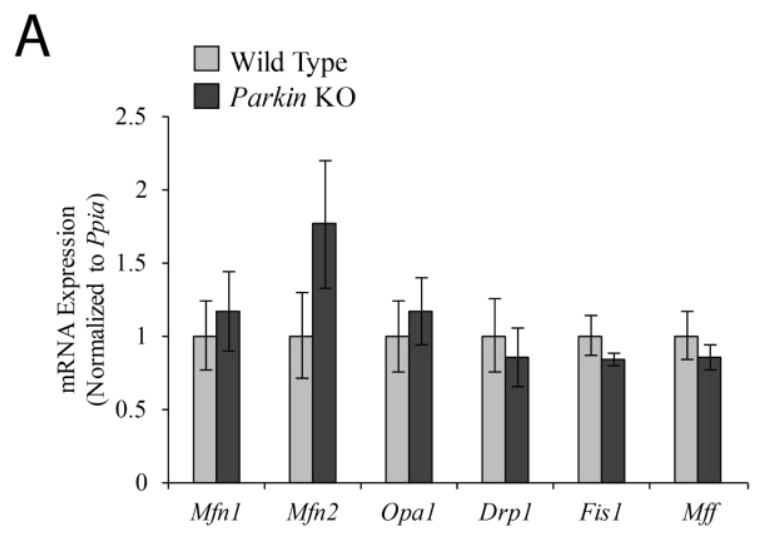

B
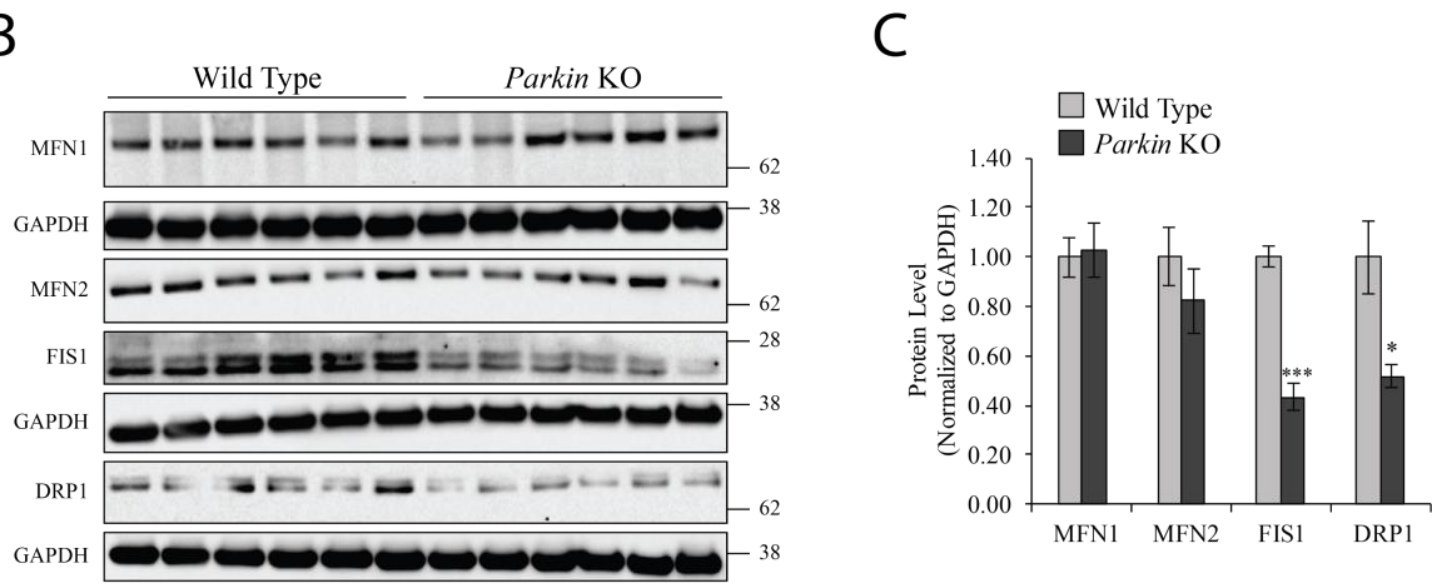

D

$E$
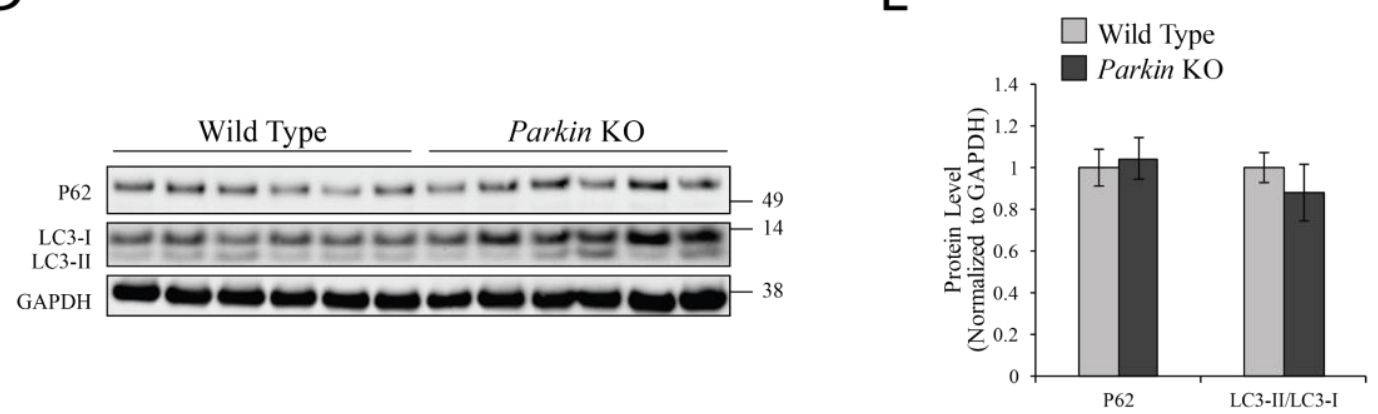

Figure 3.17: Parkin KO mice display impaired mitochondrial turnover, while autophagy remains unaltered, in muscle

A) Graph showing qPCR analysis mRNA expression of mitochondrial fusion (Mfnl, Mfn2, Opal) and fission (Drpl, Fis 1, Mff) marker mRNA expression in wild type and Parkin KO Gas muscle ( $\mathrm{n}=6$ ). B) Western Blot analysis of MFN1, MFN2, FIS1 and DRP1 in wild type and Parkin KO Gas muscle. The levels of GAPDH were assessed as a loading control. C) Graph showing quantification of MFN1, MFN2, FIS1 and DRP1 protein levels, normalized to GAPDH, in arbitrary units $(n=6)$. D) Western blot analysis of P62, LC3-I and LC3-II protein levels in wild type and Parkin KO Gas muscle. The levels of GAPDH were assessed as a loading control. E) Graph showing quantification of P62 protein levels and the level of LC3-II/LC3-I, normalized to GAPDH, in arbitrary units $(\mathrm{n}=6)$. All graphs show mean \pm s.e.m. ${ }^{*} \mathrm{P}<0.05, * * * \mathrm{P}<0.001$ (Student's t-test). 


\subsection{Knock out of Parkin results in reduced mitochondrial function in primary myotube cultures}

Next, mitochondrial function in primary myotube cultures derived from wild type and Parkin KO mice was measured through real-time assessment of OCR using the Seahorse $\mathrm{XF}^{\mathrm{e}} 24$ extracellular flux analyzer. An overall reduction in real-time oxygen consumption rate was apparent in Parkin KO myotubes (Fig. 3.18A). Moreover, a significant reduction in basal, maximal, ATP-linked and non-mitochondrial respiration was noted in Parkin KO myotubes (Fig. 3.18B). In addition, a significant reduction in spare respiratory capacity and OCR due to proton leak was further observed in Parkin KO myotubes, when compared to wild type controls (Fig. 3.18B). Consistent with impaired mitochondrial function, increased levels of ROS (Fig. 3.18C) and active pAMPK protein (Fig. 3.18D \& 3.18E) were detected in primary myoblasts harvested from Parkin KO mice, when compared to wild type controls, although the increase in p-AMPK was not statistically significant (Fig. 3.18E). Taken together, these data suggest that while mitochondrial number remains unchanged, mitochondrial function is impaired in skeletal muscle of Parkin KO mice. 
A

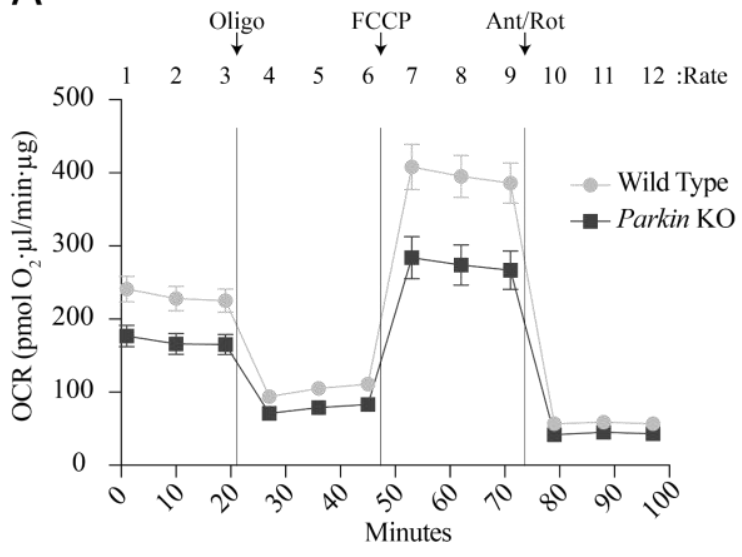

C

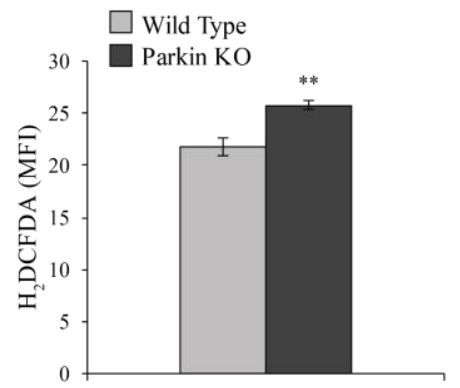

D

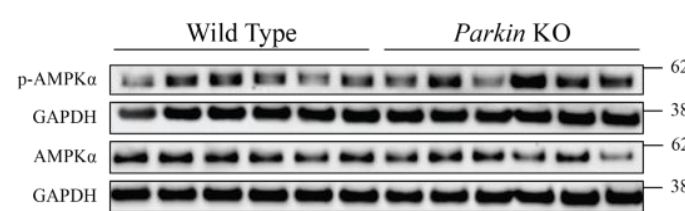

B

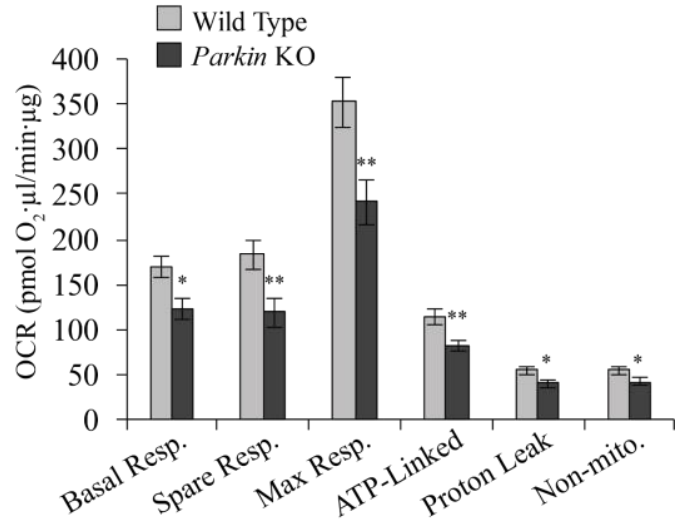

E

$\square$ Wild Type

$3.00 \square$ Parkin KO

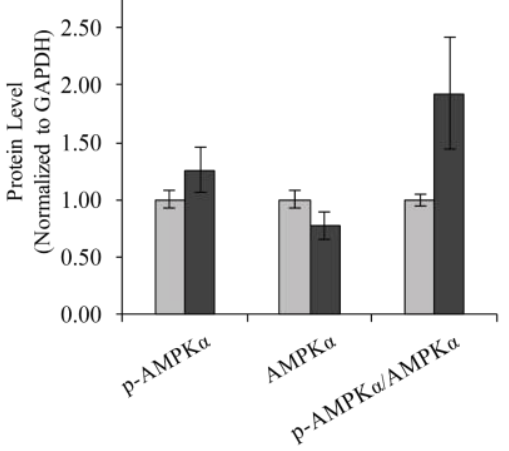


Figure 3.18: Knock out of Parkin results in reduced mitochondrial function in primary myotube cultures

A) Graph showing the real-time OCR measurement of $72 \mathrm{~h}$ differentiated primary myotubes isolated from wild type and Parkin KO hind limb muscle, as assessed by the Seahorse $\mathrm{XF}^{2} 24$ extracellular flux analyzer $(\mathrm{n}=9)$. Time points where Oligomycin (Oligo), FCCP and Antimycin/Rotenone (Ant/Rot) were injected (arrows) and the rate number where each OCR was measured are indicated. B) Graph showing quantification of basal, spare, maximal, ATP-linked and non-mitochondrial respiration, and respiration due to proton leak in $72 \mathrm{~h}$ differentiated primary myotubes isolated from wild type and Parkin KO hind limb muscle. All OCR values were normalized to total protein $(\mathrm{n}=9)$. C) Graph showing the levels of Reactive Oxygen Species (ROS), as measured by $\mathrm{H}_{2}$ DCFDA fluorescence, in $72 \mathrm{~h}$ differentiated primary myotubes isolated from wild type and Parkin KO hind limb muscle $(\mathrm{n}=4)$. D) Representative images of western blot analysis of phosphorylated (p-AMPK $\alpha$ ) and total AMPK $\alpha$ protein levels in wild type and Parkin KO Gas muscle. The levels of GAPDH were assessed as a loading control $(\mathrm{n}=6)$. E) Graph showing quantification of p-AMPK $\alpha$, total AMPK $\alpha$, and the ratio of p-AMPK $\alpha /$ total AMPK $\alpha$, normalized to GAPDH, in arbitrary units $(n=6)$. Data represent mean \pm s.e.m. ${ }^{*} \mathrm{P}<0.05,{ }^{*} \mathrm{P}<0.01$ (Student's t-test). 


\subsection{Absence of Parkin results in reduced body weight, decreased skeletal muscle weights and myofiber atrophy in mice}

As knock down of Parkin on myotubes resulted in myotubular atrophy, phenotype of Parkin KO mice was next analyzed. Parkin KO mice have significantly reduced total body weight at 4 weeks of age, when compared to age-matched wild type mice controls (Fig. 3.19A). To confirm absence of Parkin in skeletal muscle tissues collected from Parkin KO mice we performed immunoblot analysis; no detectable levels of PARKIN protein were observed in M. quadriceps (Quad), M. gastrocnemius (Gas), M. tibialis anterior (TA), M. soleus (Sol) and M. extensor Digitorum longus (EDL) hind limb muscles (Fig. 3.19B). Further characterization of hind limb skeletal muscle tissue weights revealed a significant reduction in the weights of all hind limb muscles analyzed in Parkin KO mice, when compared to wild type controls (Fig. 3.19C \& Fig. 3.19D). Consistent with this, subsequent histological analysis (Fig. 3.19E) of myofiber crosssectional area (CSA) in OCT embedded H\&E stained TA muscles, revealed a reduction in TA myofiber CSA distribution (Fig. 3.19F) and average myofiber CSA (Fig. 3.19G) in Parkin $\mathrm{KO}$ mice, when compared to wild type control. These data suggest that loss of Parkin results in reduced skeletal muscle mass in vivo. 


\subsection{The myofiber atrophy phenotype observed in Parkin KO muscle tissue may be, at least in part, due to increased MURF1 levels}

Next, to determine whether the atrophy phenotype observed in Parkin KO mice resulted from activation of the Akt-FoxO pathway, the levels and phosphorylation status of FOXO1 and FOXO3a were assessed. Western blot analysis revealed that, although the levels of phosphorylated FOXO1 (p-FOXO1) and total FOXO1 were significantly reduced, the ratio of p-FOXO1/FOXO1 remained unaltered, in Parkin KO Gas muscle (Fig. 3.20A). A significant reduction in the protein levels of phosphorylated FOXO3a (p-FOXO3a) was also noted in Parkin KO Gas muscle tissue (Fig. 3.20B). However, the levels of total FOXO3a and the ratio of p-FOXO3a/FOXO3a were comparable between Parkin KO Gas muscle and wild type controls (Fig. 3.20B). Importantly, we observed increased expression of both Murfl and Atrogin-1 in Parkin KO Gas muscle, although the increase was not statistically significant (Fig. 3.20C). In agreement with in vitro observations, western blot analysis revealed a trend towards increased levels of MURF1 protein in Parkin KO Gas muscle, albeit not statistically significant (Fig. 3.20D \& 3.20E), further suggesting that MURF1 may play a role in the myofiber atrophy phenotype observed in Parkin KO mice. 
A

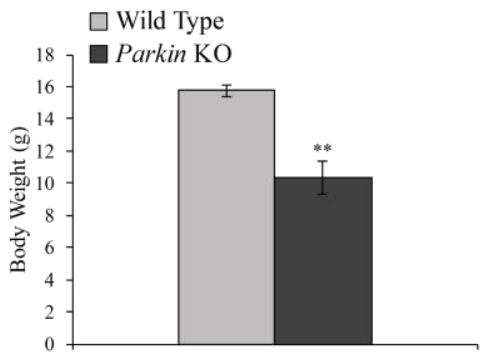

C

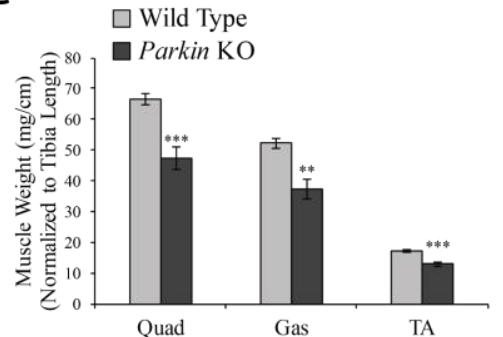

F

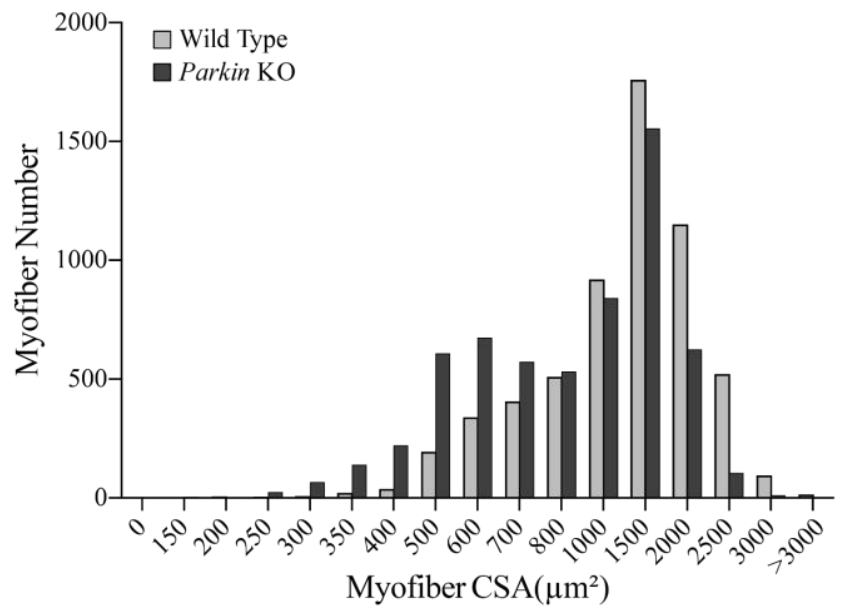

E

Wild Type

D

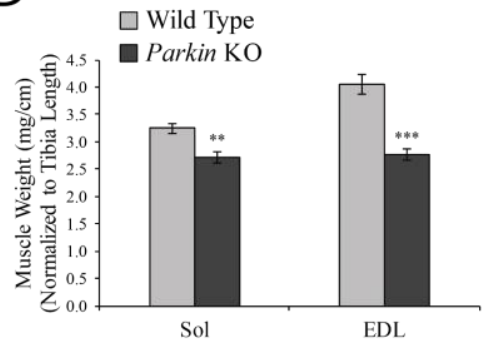

G

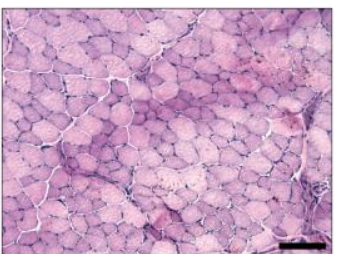

Parkin KO

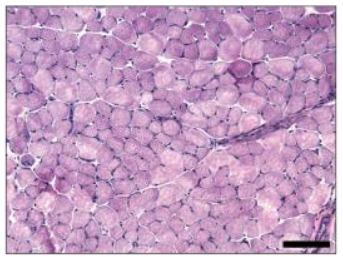

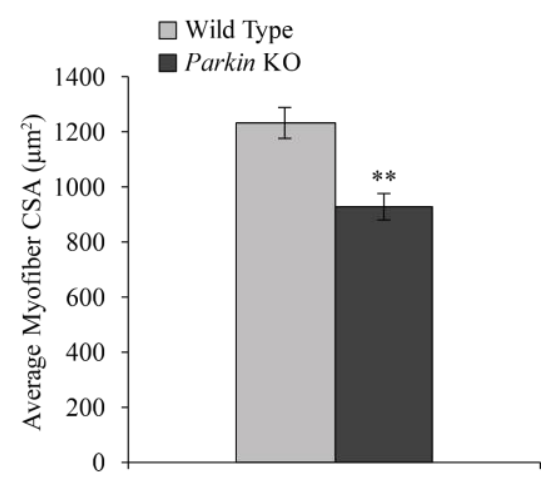


Figure 3.19: Four-week-old Parkin KO mice have reduced muscle weights and myofiber size

A) Graph showing average body weights of 4-week-old wild type and Parkin KO mice $(\mathrm{n}=6)$. B) Representative images of western blot images showing PARKIN protein levels in wild type and Parkin KO M. Quadriceps (Quad), M. Gastrocnemius (Gas), M. Tibialis anterior (TA), M. Soleus (Sol) and M. Extensor Digitorum longus (EDL) muscles. The levels of GAPDH were assessed as a loading control $(\mathrm{n}=6)$ C) Graph showing weights of Quad, Gas and TA hind limb muscles of wild type and Parkin KO mice. All hind limb muscle weights were normalized to tibia length $(\mathrm{n}=6)$. D) Graph showing weights of Sol and EDL hind limb muscles of wild type and Parkin KO mice. All hind limb muscle weights were normalized to tibia length. E) Representative images of hematoxylin and eosin (H\&E) stained M. Tibialis anterior (TA) muscle sections of 4-week-old wild type and Parkin KO mice. Scale bars represent $100 \mu \mathrm{m}$. F) Graph showing the distribution of myofiber cross-sectional area in 4-week-old wild type and Parkin KO mice TA muscles $(\mathrm{n}=6)$. G) Graph showing average myofiber crosssectional area of 4-week-old wild type and Parkin KO mice TA muscles $(\mathrm{n}=6)$. Graphs represent mean \pm s.e.m. ${ }^{* *} \mathrm{P}<0.01$ and ${ }^{* * *} \mathrm{P}<0.001$ (Student's t-test). 
A

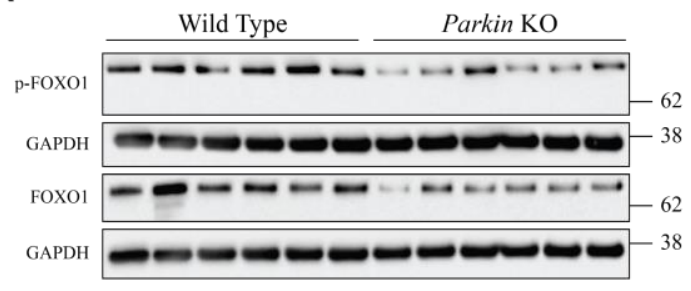

$\square$ Wild Type

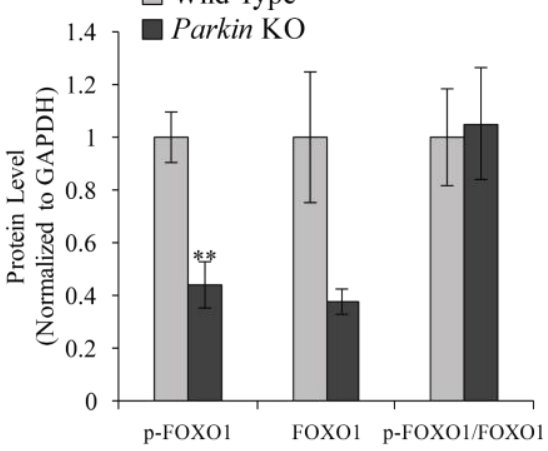

C

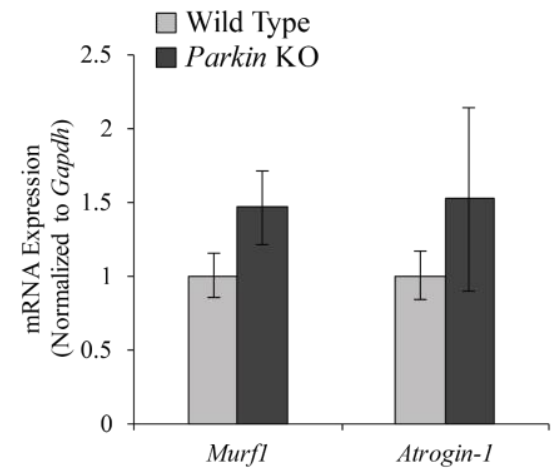

D

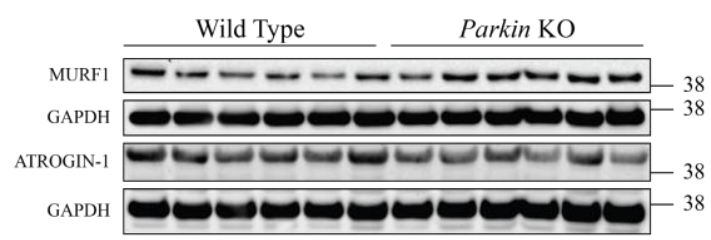

B

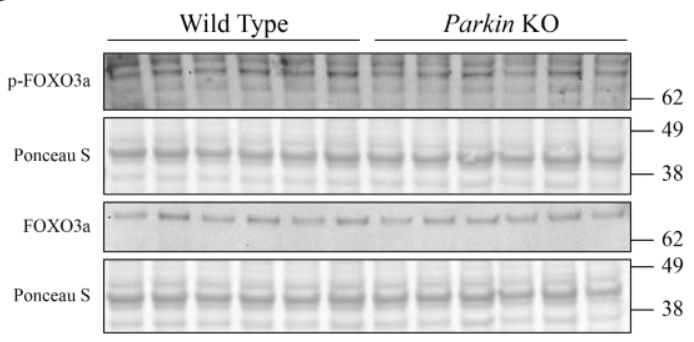

$\square$ Wild Type

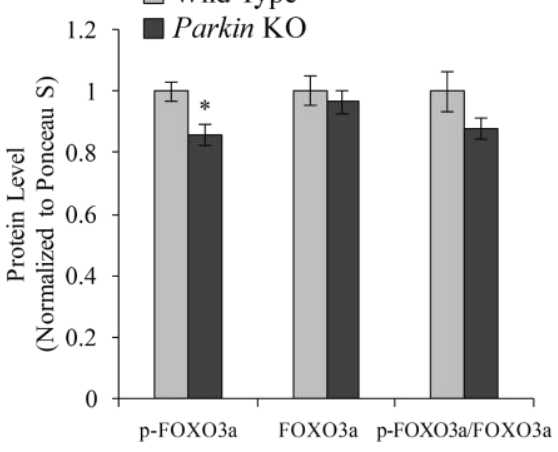

E

$\square$ Wild Type

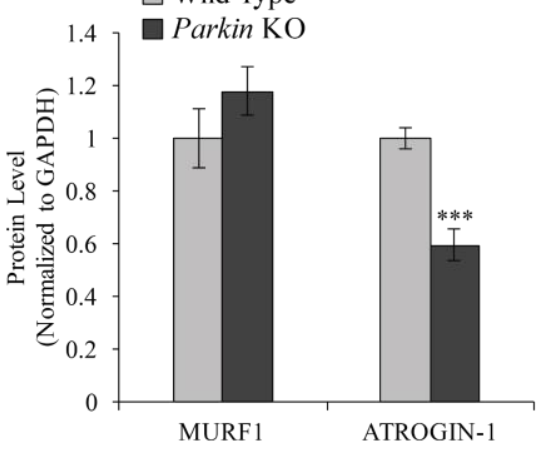


Figure 3.20: Knock out of Parkin results in moderate but not significant increase in MURF1 protein levels in Gas muscle

A) Top, Representative images of western blot analysis of phosphorylated FOXO1 (pFOXO1) and total FOXO1 in wild type and Parkin KO Gas muscle. The levels of GAPDH were assessed as a loading control $(n=6)$. Bottom, Graph showing quantification of p-FOXO1, total FOXO1 and p-FOXO1/total FOXO1 levels, normalized to GAPDH, in arbitrary units $(\mathrm{n}=6)$. B) Top, Representative images of western blot analysis of phosphorylated FOXO3a (p-FOXO3a) and total FOXO3a in wild type and Parkin KO Gas muscle. The levels of Ponceau S were assessed as a loading control $(n=6)$. Bottom, Graph showing quantification of p-FOXO3a, total FOXO3a and p-FOXO3a/total FOXO3a levels, normalized to Ponceau S, in arbitrary units. (n=6). C) Graph showing qPCR analysis of atrogenes Murf1 and Atrogin-1 in wild type and Parkin KO Gas muscle $(\mathrm{n}=6)$. D) Representative images of western blot analysis of MURF1 and ATROGIN-1 protein levels in wild type and Parkin KO Gas muscle. The levels of GAPDH were assessed as a loading control $(\mathrm{n}=6)$. E) Graph showing quantification of MURF1 and ATROGIN-1 protein levels, normalized to $\mathrm{GAPDH}$, in arbitrary units $(\mathrm{n}=6)$. Data represent mean \pm s.e.m. $* \mathrm{P}<0.5, * * \mathrm{P}<0.01$, $* * * \mathrm{P}<0.001$ (Student's t-test). 


\subsection{Genes involved in metabolic pathways are downregulated in Parkin KO}

\section{skeletal muscle}

To further understand the skeletal muscle phenotype observed in Parkin KO mice, microarray analysis was performed. Microarray analysis of RNA isolated from skeletal muscle of 3-month-old Parkin KO and age-matched wild type controls revealed several significantly ( $\mathrm{p}<0.05)$ up $(\geq 1.5$ fold) and down $(\leq 0.667$ fold $)$ regulated genes. Subsequent KEGG pathway analysis of differentially regulated genes using DAVID pathway analysis tool revealed that nitrogen metabolism was the most significantly regulated pathway, with differential expression of 6 carbonic anhydrase genes (Car2, Car3, Car4, Car12, Car14, Car15) noted (Table 3.1). It is noteworthy to that genes involved in cytoskeleton structure $(p$-value $=0.002)$ and focal adhesion $(p$-value $=0.01)$ are significantly differentially regulated in Parkin KO Gas muscle, as compared to wild type controls, indicating that loss of Parkin may lead to alterations in cytoskeletal structure in vivo (Table 3.1). Consistent with observations made both in vivo and in vitro, genes associated with the AMPK signalling pathway tended to increase in Parkin KO muscle ( $p$-value $=0.015$ ), further underscoring the identified deficiencies in mitochondrial function in Parkin KO mice (Table 3.1). Given that mitochondria have calcium buffering capacity in the cell, it is worth noting that genes involved in calcium signalling were predominantly decreased in Parkin KO muscle tissue ( $p$-value=0.21). In addition, significant differential regulation of genes involved in fatty acid metabolism ( $p$-value $=0.004)$, PPAR signalling $(p$-value $=0.01)$ (which regulates utilization of fatty acids) and biosynthesis of unsaturated fatty acids ( $p$-value $=0.047)$ underscores the importance of Parkin for fatty acid utilization, metabolism and biosynthesis (Table 3.1). 


\subsection{Expression of several ubiquitin E3 ligases and E2 ligases are increased in}

\section{Parkin KO skeletal muscle}

To further identify the molecular mechanism(s) behind the myofiber atrophy phenotype observed in Parkin KO mice, microarray data was scanned to identify differentially expressed genes involved in the ubiquitin proteasome system. Subsequent analysis revealed increased expression of several E3 ubiquitin ligases, notably Trim59, Trim43b, Rnf144a, Zfp598, Traip and Fbxl22, the deubiquitinating enzyme Usp18 and the E2 conjugating enzyme Ube2c (Table 3.2). Next, we performed qPCR analysis to validate the changes in E2 and E3 enzyme expression in Parkin KO Gas muscle. Results confirmed significant upregulation of Trim59, Rnf144a, Zfp598 and Fbxl22 and the ubiquitin conjugating enzyme Ube $2 c$ in Parkin KO Gas muscle (Table 3.2), indicating that additional components of the ubiquitin proteasome system may pay a role in the atrophy phenotype observed in Parkin KO skeletal muscle. 


\begin{tabular}{|c|c|c|c|c|c|c|}
\hline Term & Count & $\%$ & $\begin{array}{c}\text { Genes } \\
\text { Downregulated }\end{array}$ & Genes Upregulated & $\begin{array}{c}\text { P- } \\
\text { Value }\end{array}$ & $\begin{array}{c}\text { Benj } \\
\text { ami } \\
\text { ni }\end{array}$ \\
\hline $\begin{array}{c}\text { Nitrogen } \\
\text { metabolism }\end{array}$ & 6 & 0.9 & $\begin{array}{c}\text { Car14, Car15, } \\
\text { Car2, Car4 }\end{array}$ & Car12, Car3 & 0.0001 & 0.03 \\
\hline $\begin{array}{l}\text { Regulation } \\
\text { of actin } \\
\text { cytoskeleton }\end{array}$ & 16 & 2.4 & Itgam, Myl10 & $\begin{array}{c}\text { Actg1, Ctnna3, } \\
\text { Cxcr4, Ezr, Itga4, } \\
\text { Mylpf, Myl9, } \\
\text { Pik3cd, Thy1, Vasp }\end{array}$ & 0.002 & 0.16 \\
\hline $\begin{array}{c}\text { Fatty acid } \\
\text { metabolism }\end{array}$ & 7 & 1 & $\begin{array}{c}\text { Acadm, Acadvl, } \\
\text { Scd3 }\end{array}$ & $\begin{array}{c}\text { Cptla, Fads2, } \\
\text { Scdl, Scd2 }\end{array}$ & 0.004 & 0.23 \\
\hline $\begin{array}{l}\text { Pathways in } \\
\text { cancer }\end{array}$ & 23 & 3.4 & $\begin{array}{c}\text { Adcy2, Ctnna3, } \\
\text { Cdkn2a, Cdkn2b, } \\
\text { Cycs, Egln3, } \\
\text { Ednrb, Fgfl, Fgfr3, } \\
\text { Foxo1, Gna12, } \\
\text { Lam2c, Plcb2, } \\
\text { Sufu, Crk } \\
\end{array}$ & $\begin{array}{c}\text { E2f2, Cxcr4, Fgfl1, } \\
\text { Gng7, Pik3cd, Ret, } \\
\text { Tceb2, Wnt4 }\end{array}$ & 0.004 & 0.2 \\
\hline $\begin{array}{c}\text { Focal } \\
\text { adhesion }\end{array}$ & 14 & 2.1 & Flnc, Myl10, Crk & $\begin{array}{c}\text { Actg1, Col5a3, } \\
\text { Coll1a1, Coll1a2, } \\
\text { Itgal1, Itga4, } \\
\text { Mylpf, } \\
\text { Myl9,Pik3cd, Vasp }\end{array}$ & 0.010 & 0.34 \\
\hline $\begin{array}{l}\text { PPAR } \\
\text { signaling } \\
\text { pathway }\end{array}$ & 8 & 1.2 & $\begin{array}{l}\text { Cd36, Acadm, } \\
\text { Sorbs1, Scd3 }\end{array}$ & $\begin{array}{l}\text { Cptla, Fads2, } \\
\text { Scd1, Scd2 }\end{array}$ & 0.010 & 0.31 \\
\hline $\begin{array}{l}\text { AMPK } \\
\text { signaling } \\
\text { pathway }\end{array}$ & 10 & 1.5 & Cd36, Foxol, Scd3 & $\begin{array}{c}\text { Pfkfb3, Cptla, } \\
\text { Ccna2, Pik3cd, } \\
\text { Ppp2r2b, Scdl, } \\
\text { Scd2 }\end{array}$ & 0.015 & 0.33 \\
\hline $\begin{array}{l}\text { Calcium } \\
\text { signaling } \\
\text { pathway }\end{array}$ & 12 & 1.8 & $\begin{array}{l}\text { Adcy2, Cacnalh, } \\
\text { Chrna7, Ednrb, } \\
\text { Gna14, Plcb2, } \\
\text { P2rx5, Slc25a5, } \\
\text { Sphk2 }\end{array}$ & Cd38, Itprl, Plcd4 & 0.021 & 0.35 \\
\hline $\begin{array}{l}\text { Rap1 } \\
\text { signaling } \\
\text { pathway }\end{array}$ & 13 & 1.9 & $\begin{array}{c}\text { Adcy2, Fgfl, Fgfr3, } \\
\text { Id1, Itgam, Plcb2, } \\
\text { Crk }\end{array}$ & $\begin{array}{l}\text { Tiam1, Actg1, } \\
\text { Drd2, Fof11, } \\
\text { Pik3cd, Vasp }\end{array}$ & 0.030 & 0.39 \\
\hline Cell cycle & 9 & 1.3 & $\begin{array}{c}C d k n 2 a, C d k n 2 b \\
P k m y t 1\end{array}$ & $\begin{array}{c}C d c 14 a, E 2 f 2, \\
C d c 20, C c n a 2, \\
\text { Ccnb2,Plk1 }\end{array}$ & 0.034 & 0.4 \\
\hline $\begin{array}{l}\text { cGMP-PKG } \\
\text { signaling } \\
\text { pathway }\end{array}$ & 11 & 1.6 & $\begin{array}{l}\text { Adcy2, Ednrb, } \\
\text { Gna12, Plcb2, } \\
\text { Slc25a5 }\end{array}$ & $\begin{array}{c}\text { Atpla4, Itprl, } \\
\text { Myl9, Pik3cd, } \\
\text { Kcnu1, Vasp }\end{array}$ & 0.035 & 0.38 \\
\hline $\begin{array}{c}\text { Insulin } \\
\text { secretion }\end{array}$ & 7 & 1 & $A d c y 2, G c k, P l c b 2$ & $\begin{array}{c}\text { Atpla4, Glp1r, } \\
\text { Pclo, Kcnul }\end{array}$ & 0.046 & 0.42 \\
\hline $\begin{array}{l}\text { Biosynthesis } \\
\text { of } \\
\text { unsaturated } \\
\text { fatty acids }\end{array}$ & 4 & 0.6 & $S c d 3$ & Fads2, Scd1, Scd2 & 0.047 & 0.42 \\
\hline $\begin{array}{l}\text { ECM- } \\
\text { receptor } \\
\text { interaction } \\
\end{array}$ & 7 & 1 & Cd36, Lamc2 & $\begin{array}{c}\text { Col5a3, Coll1a1, } \\
\text { Coll1a2, Itga11, } \\
\text { Itga4 }\end{array}$ & 0.05 & 0.41 \\
\hline
\end{tabular}


Table 3.1: KEGG pathway analysis of differentially expressed genes in Parkin KO Gas muscle

Table Table showing Kyoto Encyclopaedia of Genes and Genomes (KEGG) pathway analysis of significantly upregulated $(\geq 1.5)$ and downregulated $(\leq 0.667)$ genes, as identified through microarray analysis, in 3-month-old Parkin KO Gas muscle as compared to age-matched wild type controls. Pathway names (Terms), number of genes (counts), percentage of total gene count in each term (\%), gene symbols, $p$-values and Benjamini-Hochberg critical values (Benjamini) are provided. 


\begin{tabular}{|c|c|c|c|c|c|c|c|}
\hline $\begin{array}{c}\text { Gene Accession } \\
\text { Number }\end{array}$ & $\begin{array}{c}\text { Gene } \\
\text { ID }\end{array}$ & Function & Structure & $\begin{array}{c}\text { Fold } \\
\text { Change } \\
\text { (Array) }\end{array}$ & $\begin{array}{c}\boldsymbol{p} \text {-value } \\
\text { (Array) }\end{array}$ & $\begin{array}{c}\text { Fold } \\
\text { Change } \\
\text { (qPCR) }\end{array}$ & $\begin{array}{c}\boldsymbol{p} \text {-value } \\
\text { (qPCR) }\end{array}$ \\
\hline NM_026785 & Ube2c & $\begin{array}{c}\text { Ubiquitin- } \\
\text { conj. } \\
\text { enzyme }\end{array}$ & UBCc & 1.89 & 0.028 & 3.19 & 0.009 \\
\hline NM_175206 & Fbxl22 & E3 ligase & F-box, LRR & 1.71 & 0.042 & 1.85 & 0.010 \\
\hline NM_080563 & Rnf144a & E3 ligase & RING, IBR & 1.59 & 0.007 & 1.66 & 0.030 \\
\hline NM_011634 & Traip & E3 ligase & RING & 1.55 & 0.003 & 1.63 & 0.397 \\
\hline NM_001170884 & Trim43b & E3 ligase & RING & 3.75 & 0.002 & 0.95 & 0.805 \\
\hline NM_025863 & Trim59 & E3 ligase & RING & 2.14 & 0.002 & 1.96 & 0.013 \\
\hline NM_183149 & Zfp598 & E3 ligase & RING, & 1.56 & 0.002 & 1.48 & 0.033 \\
\hline NM_011909 & Usp18 & $\begin{array}{c}\text { De-ubiq. } \\
\text { enzyme }\end{array}$ & N/A & 1.67 & 0.008 & 1.35 & 0.229 \\
\hline
\end{tabular}

Table 3.2: Genes involved in the ubiquitin proteasome system are upregulated in Parkin KO Gas muscle

Tab Table showing fold change differences in gene expression of the ubiquitinconjugating enzyme Ube2c, E3 ligases (Fbxl22, Rnfl44a, Traip, Trim43b, Trim59, Zfp598) and the deubiquitinating enzyme Usp18 in Parkin KO Gas muscle as compared to wild type control, as assessed by microarray (Array) and qPCR analysis. Gene accession numbers and calculated $p$-values for microarray and qPCR analysis are provided. Domains (Structure) within each protein sequence were identified using SMART (a Simple Modular Architecture Research Tool). (UBCc: Ubiquitinconjugating enzyme E2 domain; LRR: Leucine-Rich-Repeat domain; RING: ReallyInteresting-New-Gene domain; RBR: RING-Between-RING domain; Znf-C2H2: Zincfinger Cys(2)His(2) domain; N/A: Not applicable (there are no hidden domains or features present). The terms Ubiquitin-conj. enzyme and De-ubiq. enzyme are used to represent Ubiquitin conjugating enzyme and Deubiquitinating enzyme, respectively. 


\section{DISCUSSION}

PD is a neurodegenerative disease that is reported to affect almost 7 million people around the globe ${ }^{234}$. Affected individuals suffer from symptoms including, tremors, bradykinesia (abnormally slow movement), hypokinesia (diminished motor/muscle activity), muscle weakness, depression and dementia ${ }^{277}$. Clinical studies have revealed systemic loss of dopaminergic neurons in substantia nigra pars compacta (SNc), which has been associated with typical motor symptoms observed in $\mathrm{PD}^{277}$. In the early 1980s, the mitochondrial dysfunction theory of PD was established, following the discovery of a parkinsonian-like phenotype among drug addicts who intravenously used a mitochondrial complex I inhibitor, MPTP ${ }^{237}$. This finding was consistent with studies that have revealed reduced complex I activity, increased mtDNA mutations and reduced expression of mitochondrial genes in $\mathrm{PD}^{278}$. After 25 years of GWAS studies, scientists have identified candidate genes that are associated with familial PD. In particular, the identification of the E3 ligase, PARKIN, as a central mitochondrial quality control protein led to further studies that focused on the role of mitochondrial function in $\mathrm{PD}^{279}$. Studies into the function of PARKIN revealed that mutations in the PARKIN gene lead to mitochondrial dysfunction, impaired mitochondrial turnover, abnormal cristae modelling and mitochondrial morphology $280,281,282$. Although it is widely known that mutations in PARKIN results in the mitochondrial dysfunction observed in PD, little is known about the role of PARKIN in skeletal muscle metabolism and maintenance of skeletal muscle mass. This thesis provides insight into the role of Parkin in skeletal muscle function using both in vitro and in vivo animal models.

\subsection{CCCP induces PINK1/PARKIN mediated mitophagy in C2C12 myotubes}

Mitochondrial uncoupling agents are commonly utilized in mitophagy research to recapitulate the reduced mitochondrial membrane potential observed prior to selective removal of mitochondria. Among the available mitochondrial uncoupling agents, CCCP has been shown to stimulate PINK1/PARKIN-mediated removal of mitochondria following dissipation of membrane potential in vitro, while other organelles remain intact $^{283,284}$. Although observations in HeLa ${ }^{199}$, HEK-293 and HCT-116 ${ }^{285}$ cells provide

compelling evidence to support that CCCP induces PINK1/PARKIN mediated mitophagy, the effect of CCCP on mitochondrial function and turnover in skeletal muscle function and growth has not been extensively studied. Analogous to previous 
studies, results presented here revealed that CCCP treatment promotes mitophagy in muscle cells through activation of the PINK1/PARKIN pathway. Importantly, we observed increased levels of active full length PINK1 protein $(63 \mathrm{kDa})$ upon CCCP treatment. This suggests that PINK1 is protected from processing through the action of the presilin-associated rhomboid-like protease (PARL) and mitochondrial processing peptidase (MPP), which proteolytically cleave PINK1 to a $53 \mathrm{kDa}$ protein ${ }^{286}$. We also observed increased translocation of PARKIN to the mitochondria, which is consistent with enhanced PARKIN function ${ }^{287}$ and induction of PINK1/PARKIN-specific mitophagy ${ }^{199}$, upon CCCP treatment (Fig. 3.1C).

Moreover, the increased recruitment of PARKIN protein to mitochondria was associated with a reduction in mitochondrial number and impaired mitochondrial function as determined through mtDNA copy number analysis and real-time measurement of mitochondrial respiration (Fig 3.2C \& 3.3A). Confocal analysis of DMSO and CCCP treated myoblasts revealed puncta formation upon CCCP treatment (Fig. 3.2E), suggesting that CCCP leads to disruption of mitochondrial architecture. In 1950, electron microscopy (EM) led to the discovery of autophagy. Thereafter, EM remained as the central technique utilized to visualize autophagic vesicles in the cell ${ }^{288}$. Although EM provides a high-resolution images of mitochondria that are engulfed by autophagosomes, it remains as a qualitative approach ${ }^{289}$. Therefore, EM not only need an objective expertise while interpreting data but also should be accompanied with a quantitative approach.

Moreover, CCCP treatment led to increased expression of ER stress response markers (Fig. 3.4A), underscoring crosstalk between mitochondria and the ER. Collectively, these results suggest that CCCP induces PINK1/PARKIN-mediated mitophagy in $\mathrm{C} 2 \mathrm{C} 12$ myotubes.

\subsection{Loss of Parkin also results in mitochondrial dysfunctional and altered mitochondrial fusion/fission in skeletal muscle}

Although main function of Parkin is to promote mitophagy by degrading mitochondrial fusion proteins ${ }^{290,216}$, recent studies have revealed that parkin overexpression protects against apoptosis-induced proteotoxicity in Zebrafish and improves mitochondrial function and extends lifespan in fly models, suggesting that selective elimination of damaged mitochondria enhances cellular homeostasis ${ }^{291,292}$. Interestingly, results presented here suggest that loss of Parkin results in maintenance 
of mitochondrial mass (Fig. 3.7C \& 3.15B), which was associated with no overt autophagy/mitophagy (Fig. $3.9 \& 3.17 \mathrm{D} \& 3.17 \mathrm{E}$ ). These data, together with the fact that loss of Parkin leads to impaired mitochondrial function (Fig 3.10A \& 3.18A), suggest that loss of Parkin results in accumulation of dysfunctional mitochondria. Although Seahorse XF Analyzer provides a high-quality assessment of mitochondrial function, it has some technical limitations. First, in this study, mitochondrial function was assessed in intact cells, since isolating mitochondria may result in contamination of mitochondria with unpredictable cellular components or damage of mitochondrial integrity. Also, since PARKIN is a cytosolic protein that is recruited to mitochondria upon reduced mitochondrial membrane potential, intact cells have been preferred to obtain mitochondrial function. However, contribution of activity of cytosolic oxygen consuming enzymes to oxygen consumption rate should be considered when intact cells are used. Nevertheless, injection of Antimycin A/Rotenone shuts down oxygen consumption due to mitochondrial respiration and reveals non-mitochondrial respiration. Ultimately, subtraction of non-mitochondrial respiration from other respiratory parameters minimizes the intervention of other oxygen consuming sources. Also, another limitation of Seahorse XF Analyzer is that Seahorse XF analyzer does not provide a direct assessment of mitochondrial complex activity. Yet mitochondria are major consumers of oxygen in the cell, oxygen consumption is not a direct measurement of mitochondrial function. A direct measurement of activity of mitochondrial complexes would provide a better evidence to suggest that loss of Parkin leads to reduced mitochondrial complex I and complex II activities.

Results presented in the current study revealed that loss of Parkin does not result in a notable increase in the levels of either mitochondrial fusion or fission markers in vitro and in vivo. However, knock down of Parkin led to a significant reduction in both mitochondrial fusion and fission protein markers in C2C12 myoblasts (Fig. 3.8B \& 3.8C). A previous study by Ishihara et al., 2009 reported that knock out of Drpl leads to a concomitant reduction in the levels of mitochondrial fusion proteins (MFN1 and MFN2), which was thought to be a compensatory mechanism for the loss of the mitochondrial fission protein $\operatorname{Drpl}^{293}$. The results from this previous study are quite consistent with the reduced DRP1, MFN1 and MFN2 protein levels observed in Parkin knock down myoblasts (Fig 3.8B \& 3.8C) and suggest that the reduced levels of MFN1 and MFN2 may be due to loss of DRP1. However, in contrast, with the exception of MFN1, knock down or loss of Parkin did not appear to dramatically alter the protein 
levels of mitochondrial fusion or fission markers in myotube cultures. (Fig. 3.8D, 3.8E, $3.15 \mathrm{~F} \& 3.15 \mathrm{G})$. Recently, it has been shown that mitophagy increases and is required during myogenic differentiation of $\mathrm{C} 2 \mathrm{C} 12$ cells $^{294}$. Given the fact that no observable deficiencies during the early stages of myogenic differentiation were noted in either Parkin knock down or knock out cultures, it is interesting to surmise that the maintenance of mitochondrial fusion and fission protein levels may form the basis of an as yet unidentified compensatory mechanism to allow normal myogenic differentiation to ensue in the absence of Parkin. However, further work will need to be undertaken to confirm this.

In contrast to the results observed in myotube cultures, western blot analysis revealed a significant reduction in protein levels of mitochondrial fission markers in Parkin KO Gas muscle, as compared to wild type controls (Fig. 3.17B \& 3. 17C). Given the fact that these experiments were performed in different model systems, namely cultured cells and whole muscle tissue, it is plausible that the disparity in levels of mitochondrial fission markers noted may be due to the inherent differences between cultured cells and muscle tissue in vivo. Previous studies have shown that parkin activates DRP1 through phosphorylation which in turn results in recruitment of DRP1 to mitochondria. In this study, DRP1 levels have been investigated in total cell lysates which may not be a direct indication of activation of DRP1. However, there are compelling evidences that phenotype observed in human cell line due to loss of PARKIN has been alleviated with DRP1 expression or worsened with loss of DRP1 suggesting that decreased DRP1 levels in Parkin-siRNA transfected myoblasts and Parkin knock out Gas muscle seem to be in line with the phenotype observed in mammalian models ${ }^{295,296}$. Although the mechanism that how DRP1 expression alleviate phenotype noted in PARKIN deficient models, results here further confirm the phenotype observed previously in different studies.

It is important to highlight that both $M f n 1$ and $M f n 2$ are required for development, which is underscored by the fact that knock out of both $M f n 1$ and $M f n 2$ is postnatally lethal in mice 297,298 . Therefore, given the fact that the Parkin KO mice used in this study are healthy and survive to a similar age as wild type controls with a slightly increase mortality rate ${ }^{299}$, it is quite possible that mechanisms may be in place to maintain the protein levels of MFN1 and MFN2 in vivo, in an attempt to maintain the viability of the mice. However, further work will need to be performed to verify this. 
In line with previous reports that have revealed reduced complex I and to some extend complex II activity in PD patients $236,237,300,301$; loss of Parkin in skeletal muscle resulted in a trend towards reduced levels of mitochondrial complex proteins, with a significant reduction in complex I (NDUFB8) and complex II (SDHB) subunits noted in Parkin KO skeletal muscle (Fig. 3.16A 3.16B). However, it is important to highlight the significant increase in the mRNA expression of several nuclear DNA-encoded mitochondrial OXPHOS genes upon knock down of Parkin (Fig. 3.7G). Previous studies have reported increased expression of mitochondrial OXPHOS genes in diseases associated with mitochondrial complex deficiencies ${ }^{302}$; suggesting that the increased mRNA expression of OXPHOS genes noted upon loss of Parkin may be a compensatory response to counteract reduced OXPHOS protein levels and impaired mitochondrial respiratory capacity observed in Parkin knock down cells.

The generation of ATP is the ultimate product of the ETC and OXPHOS in mitochondria. However, mitochondria and the process of OXPHOS results in the formation of unwanted by-products, including ROS, which pose potential risks to nucleic acids, proteins and lipids. To counteract this, mitochondria have developed different responses to lower the levels of cellular $\operatorname{ROS}^{303}$. Consistent with mitochondrial dysfunction noted upon loss of Parkin, increased levels of cellular ROS in both Parkin knock down and knock out models was observed (Fig 3.10C \& 3.18C). A recent study has revealed increased ROS levels following knock down of the mitochondrial complex I assembly factor NDUFAF $1^{304}$, which agrees with the changes observed in the current study (Fig 3.7D \& 3.7E \& 3.16A \& 3.16B). Therefore, it is interesting to surmise that the reduced levels of mitochondrial OXPHOS proteins observed in the absence of Parkin may lead to increased cellular ROS levels and mitochondrial dysfunction in muscle. It is noteworthy to mention that elevated ROS levels have previously been associated with induction of skeletal muscle atrophy ${ }^{305-307}$. Therefore, it is quite possible that the increased cellular levels of ROS observed upon loss of Parkin may also contribute to the atrophy phenotype observed. In this thesis DCFDA has been used to detect cellular ROS in Parkin-siRNA transfected myoblasts or Parkin knock out primary myoblasts. DCFDA is the most commonly used probe to detect cellular ROS. DCFDA is able to penetrate through plasma membrane and detect several ROS such as $\mathrm{H}_{2} \mathrm{O}_{2}, \cdot \mathrm{OH}$ and $\mathrm{O}_{2}{ }^{-308}$. Given that DCFDA does not specifically detect ROS generated by mitochondria, other fluorescent probes, mitoSOX or mitoTEMPO would have given a more precise assessment of mitochondrial ROS. However, one limitation of those dyes 
that delivery of the probes are facilitated with lipophilic cation, triphenylphosphonium cation $\left(\mathrm{TPP}^{+}\right)$, which perturbates mitochondrial membrane potential thereby ROS production $^{309}$. To overcome such limitations due to specificity of the probes, mitochondrial scavenging enzymes should be assessed. Since superoxide ions are converted to $\mathrm{H}_{2} \mathrm{O}_{2}$ through activity of SOD2 (Reviewed in Section 1.4.1.2), protein levels of SOD2 would reveal whether loss of Parkin results in an increased ROS response in mitochondria.

AMPK has plethora of functions in the cell. However, the main function of AMPK is thought to be linked to maintaining energy homeostasis in the cell through sensing AMP:ATP and ADP:ATP ratios. Depletion of energy stores leads to activation of AMPK, which switches on catabolic pathways while inhibiting anabolic processes that consume $\mathrm{ATP}^{310}$. Importantly, previous studies have shown that activation of the AMPK pathway alleviates atrophy of dopaminergic neurons observed in $\mathrm{PD}^{311,312}$, while inhibition of AMPK has a neuroprotective role during the progression of $\mathrm{PD}^{313}$. Data presented here (using both in vitro and in vivo models) reveal that loss of Parkin leads to increased levels of active phosphorylated AMPK $\alpha$. Therefore, it is interesting to surmise that mitochondrial dysfunction in skeletal muscle, due to loss of Parkin, results in energy deprivation, subsequent activation of AMPK and increased catabolism, which is quite consistent with the previously described function of AMPK in maintaining energy homeostasis ${ }^{314}$. Collectively, these data suggest that loss of Parkin leads to impaired mitochondrial turnover (mitophagy) and mitochondrial dysfunction in skeletal muscle, which further underscores the importance of Parkin function to skeletal muscle metabolism.

\subsection{CCCP treatment results in atrophy phenotype in vitro}

Previous studies have observed mitochondrial dysfunction in glucocorticoid- and disuse-induced atrophy ${ }^{167,315,316}$. Consistent with this, data presented here revealed that CCCP treatment resulted in the development of myotubular atrophy in C2C12 muscle cells. Skeletal muscle atrophy has been shown to be triggered by increased expression of atrophy-related genes (atrogenes) through the activity of FoxO transcription factors $^{273}$. It is widely accepted that different muscle wasting conditions, ranging from cancer cachexia to denervation, are mediated through a common Akt-FoxO-dependent mechanism. Specifically, a reduction in the phosphorylation of AKT, concomitant with 
a decrease in the phosphorylation of FOXO proteins, results in sequestration of FOXO proteins to the nucleus, which in turn leads to increased expression of atrogenes ${ }^{273}$. Surprisingly, results revealed that CCCP treatment led to increased levels of p-AKT in $\mathrm{C} 2 \mathrm{C} 12$ myotube cultures, despite the fact that a significant reduction in levels of active phosphorylated FOXO3a was observed (Fig. 3.6C \& 3.6D). Importantly, a recent study by Guha et al., 2010 has shown the levels of p-AKT increase in response to CCCPinduced mitochondrial dysfunction ${ }^{317}$. Moreover, it was proposed that CCCP-mediated activation of AKT has an important role in facilitating the mitochondrial respiratory stress response ${ }^{317}$. Taken together, these data suggest that the increased levels of p-AKT observed in the current study may be attributed to the impaired mitochondrial function noted upon CCCP treatment. Moreover, although nuclear translocation of FoxO is mainly regulated by Akt, Akt-independent regulation of FoxO through Sirtuin1 $(\mathrm{SIRT} 1)^{318}$, heat shock protein $70(\mathrm{HSP} 70)^{287}$ or $14-3-3$ signaling protein ${ }^{319}$ has been reported. Thus, it is quite possible that activation of FoxO3 in response to CCCP treatment may occur independent of Akt. Nevertheless, qPCR analysis revealed significantly increased expression of the FoxO downstream target atrogenes Murfl and Atrogin-1 in response to CCCP treatment (Fig. 3.6E). Collectively, these data suggest that CCCP-induced mitochondrial dysfunction, impaired mitochondrial turnover and subsequent disruption of energy homeostasis results in loss of skeletal muscle mass in vitro through activation of FoxO3 and downstream atrogenes.

\subsection{Loss of Parkin Leads to skeletal muscle atrophy in vitro and in vivo}

Longstanding clinical studies have reported muscle weakness, resistance to exercise and fatigue in PD patients ${ }^{320}$. Similarly, parkin knock out fly mutants exhibit locomotor defects ${ }^{174}$. In line with the impaired skeletal muscle function described above, a notable atrophy phenotype in both Parkin knock down and knock out models was observed. Immunoblotting analysis revealed that p-FOXO1/FOXO1 and p-FOXO3a/FOXO3a ratios were unaltered both in vivo and in vitro. However, interestingly, protein levels of MURF1 showed a significant increase upon knock down of Parkin in vitro and this increase was reflected in Parkin KO Gas muscle, although the increase was not statistically significant. It is also important to note a remarkable increase in mRNA expression of Atrogin-1 and Murfl in Parkin KO Gas muscle as compared to control. Consistent with the increased atrogene expression, Parkin knock down resulted in a significant increase in chymotrypsin-like and caspase-like proteolytic activities, further 
indicating that loss of Parkin leads to increased protein degradation. Taken together, these data suggest that loss of Parkin results in skeletal muscle atrophy phenotype both in vivo and in vitro through activation of the muscle-specific E3 ligase MuRF1. However, it is important to highlight that loss of Parkin only resulted in a modest increase in MuRF1 protein levels and mRNA expression; suggesting that additional E3 ligases may contribute to the degradation of myofibrillar proteins and thus, the skeletal muscle atrophy noted in response to loss of Parkin.

To further identify additional mechanisms that may contribute to the atrophy phenotype observed in Parkin KO skeletal muscle, microarray analysis was performed. Subsequent pathway analysis (using DAVID online functional annotation tool) of the microarray data revealed that nitrogen metabolism was the most significantly regulated pathway in Parkin KO Gas muscle, as compared to wild type controls, with the lowest $p$-value (0.0001). A significant increase in the expression of the Car3 gene was noted in Parkin KO Gas muscle, when compared to wild type controls (Table 3.1). Interestingly, muscle-specific carbonic anhydrase-3 (Car3) mRNA expression has been shown to be upregulated in mice and chicken skeletal muscle dystrophy models ${ }^{321,322}$ and in COPD-induced skeletal muscle atrophy patients ${ }^{323}$. Also, elevated CAR3 levels in fetal plasma has been proposed to be a marker for the early diagnosis of DMD in the developing fetus ${ }^{324}$. The function of CAR3 has been postulated to act as cellular antioxidant in skeletal muscle, which suggests that the increased Car3 expression observed in Parkin KO Gas muscle may be due to elevated ROS levels ${ }^{325}$. It is important to note that genes involved in AMPK signaling are mainly upregulated in Parkin KO Gas muscle, which is consistent with the activation of AMPK observed in the in vivo and in vitro loss of Parkin models used in the current study. Interestingly, recent studies have revealed that AMPK regulates the activity of FoxO transcription factors, which are upstream regulators of atrogenes, such as Murfl, which further promote degradation of myofibrillar proteins and skeletal muscle atrophy ${ }^{326,327}$. Therefore, it is important to speculate that the increased activation of AMPK observed in the current study may be a contributing factor towards the muscle atrophy phenotype noted in loss of Parkin models.

Differential regulation of pathways involved in fatty acid metabolism ( $p$ value $=0.004)$, PPAR signaling $(p$-value $=0.01)$ and biosynthesis of unsaturated fatty acids ( $p$-value $=0.047$ ) in Parkin KO Gas muscle underscores the role of Parkin in fatty acid metabolism. Indeed, previous studies have reported that Parkin KO mice are 
resistant to fat accumulation and insulin resistance due to high fat diet (HFD), due to the fact that Parkin positively regulates fat uptake in mice 328,329 .

Further analysis of protein class using PANTHER pathway analysis, revealed UPSrelated genes differentially regulated in Parkin KO Gas muscle. Microarray analysis showed a significant increase in $U b e 2 c$ gene expression, which is further validated by qPCR analysis. Ube $2 c$ is an E2-conjugating enzyme that has been associated with degradation of mitotic cyclins during cell division ${ }^{330,331}$. Also, high expression of Ube $2 c$ has been reported in various human cancer cell lines, indicating that $U b e 2 c$ may play a role in the proteasome-dependent cell cycle regulation in Parkin KO skeletal muscle ${ }^{332}$. Anaphase promoting complex/cyclosome (APC/C) forms a complex with either cadherin-1 (CDH1) or cell division cycle 20 (CDC20) and degrades mitotic substrate through the partner E2 enzymes, UBE2C and UBE2S ${ }^{333}$. Lee et al. has revealed that PARKIN substitutes APC/C complex, hence promotes degradation of cell cycle proteins, when a serine/threonine kinase, Polo-like kinase-1 (PLK1) phosphorylates PARKIN at Ser378 334 . Interestingly, besides increased expression of $U b e 2 c$, microarray analysis has shown increased expression of other partners of PARKIN in cell cycle regulation, such as Cdc20, Plk1 (Table 3.1), which underscores the role of Parkin in regulation of cell division through activation of ubiquitin-dependent proteolysis. Moreover, microarray analysis and qPCR analysis revealed increased expression of Fbox and leucine-rich repeat protein-22 (Fbxl22) by 1.71-fold ( $p$-value: 0.042) and 1.85fold ( $p$-value: 0.01), respectively. Importantly, a recent study showed that Fbxl22 promotes degradation of critical sarcomeric proteins, which makes Fbxl22 a strong E3 ligase candidate that may be responsible for the atrophy phenotype observed in Parkin KO skeletal muscle ${ }^{335}$. Given that PARKIN is a RBR E3 ligase, it is interesting to note increased expression of another RBR E3 ligase, Rnf144a, in Parkin KO Gas muscle, when compared to wild type controls (Table 3.2). Previous studies have uncovered a role for RNF144A in apoptosis induced upon DNA damage and concomitant activation of $\mathrm{p} 53^{336,337}$. The expression of two tripartite motif family proteins, Trim $43 \mathrm{~b}$ and Trim59, was also increased in Parkin KO Gas muscle, however, to date their function in regulation of skeletal muscle mass remains to be determined. Upregulation of the deubiquitinating enzyme, Usp 18, has been implicated in activating the immune response upon viral infection ${ }^{338}$ and in response to exposure to interferons ${ }^{339}$ and bacterial lipopolysaccharides (LPS) ${ }^{340}$. Although Usp18 function in skeletal muscle remains to be studied, Peng et al., 2016 have reported upregulation of USP18 in muscle fibers 
obtained from patients suffering from dermatomyositis (DM) ${ }^{341}$. Collectively, microarray data underscores the potential involvement of the ubiquitin-proteasome pathway in the skeletal muscle atrophy phenotype observed in Parkin KO mice.

Given that Parkin knock out mice do not show signs of nigral degeneration as reported in human PD patients, it is noteworthy that loss of Parkin leads to reduced synaptic excitability in nigral neurons (Reviewed in Section 1.5). Impaired mitochondrial function and reduced muscle mass in skeletal muscle of Parkin knockout mice can be attributed to degeneration of neurons in $\mathrm{SNc}$ which resides in midbrain and controls reward and movement ${ }^{342}$. However, whether motor neurons controlled by those in SNc are denervated or not should be further investigated. Moreover, loss of Parkin may lead to an intrinsic alteration in skeletal muscle, which in turn results in impaired mitochondrial respiration and reduced muscle mass. Similarly, previous study have reported that PARKIN mutated fibroblasts collected from PD patients display reduced mitochondrial function and disrupted mitochondrial network further pointing out spatial effect of PARKIN deficiency.

\subsection{Conclusion}

Here I investigated the role of Parkin in skeletal muscle function and mass. In this study, results revealed that mitophagy is regulated through the activity of the PINK1/PARKIN pathway in skeletal muscle. In addition, results clearly show that loss of Parkin results in reduced mitochondrial respiration, elevated cellular ROS levels and impaired mitochondrial turnover, both in vitro and in vivo. In agreement with impaired mitochondrial function, the levels of active AMPK protein were increased, which is consistent with skeletal muscle loss observed in Parkin knock down myotubes. Moreover, notable myotubular atrophy was observed in both Parkin-siRNA transfected myotubes and Parkin KO skeletal muscle, suggesting a role for PARKIN in regulating skeletal muscle mass. Based on the data presented here, it is proposed that the skeletal muscle atrophy, observed upon loss of Parkin, is partly mediated by the muscle-specific E3 ligase, Murf1, which is quite consistent with the increased proteasome activity noted. Pathways that were differentially regulated in Parkin KO Gas muscle and candidate proteins that may promote UPS-mediated degradation of myofibrillar proteins in the absence of Parkin were further identified. Collectively, these data here underscore an emerging function for Parkin in the maintenance of skeletal muscle function and mass. 


\section{FUTURE PROSPECTS}

Parkinson's Disease (PD) is associated with involuntary tremors, muscle weakness, abnormal gait and postural instability ${ }^{277}$. Although PD was first described 200 years $\mathrm{ago}^{343}$, the complex pathophysiology of PD still puzzles scientists today. To date, genetic studies have associated mutations in five genes (PARKIN (PARK2), PINK1 (PARK6), DJ-1 (PARK7), SCNA, LRRK2) with the familial form of $\mathrm{PD}^{239}$. Since mutations in the PARKIN gene account for the majority of familial $\mathrm{PD}^{239}$ and moreover, given the fact that PARKIN plays a central role in the mitochondrial quality control mechanisms ${ }^{344}$; mitochondrial dysfunction has been implicated in PD. Although it is clear that PARKIN has important roles in controlling mitochondrial quality, and that PD is associated with muscle weakness and loss of muscle mass, the role of PARKIN in regulating skeletal muscle growth and metabolism has remain unstudied. In this thesis, I suggest that loss of Parkin results in mitochondrial dysfunction, thereby leading to the development of skeletal muscle atrophy. Although results presented in this thesis suggests that Murfl may contribute to the atrophic phenotype observed upon loss of Parkin, microarray analysis revealed several additional E3 ligases significantly upregulated in Parkin KO Gas muscle, when compared to wild type controls. To further characterize the molecular mechanism behind the atrophy phenotype observed in the in vitro and in vivo loss of Parkin models, it would be interesting to knock down Murfl and additional E3 ligases, such as Fbxl22, in Parkin KO skeletal muscle using AAV6mediated intramuscular administration, which has been shown to effectively target skeletal muscle tissue ${ }^{345}$. Subsequently, I would perform skeletal muscle histology and assess for changes in myofiber size, measure the protein and mRNA levels of muscle wasting markers and determine proteasomal activity in muscle tissue. In addition, it would be interesting to assess mitochondrial function and number in Parkin $\mathrm{KO}$ mice.

Previous studies have shown that the indirect fly muscle (IFM) of pinkl and parkin loss-of-function mutant fly display fragmented mitochondria, which is rescued upon overexpression of the mitochondrial fission marker $d r p 1^{346-348}$. Consistent with previous reports, in this thesis, I found that loss of Parkin results in reduced levels of the mitochondrial fission markers DRP1 and FIS1 in vivo and that this was associated with impaired mitochondrial turnover and mitochondrial dysfunction. Therefore, I would like to perform rescue experiments to investigate whether overexpression of Drpl and Fis 1 
can improve mitochondrial turnover and function and whether this has any impact on the muscle atrophy phenotype noted in Parkin KO skeletal muscle.

A recent study has suggested Parkin KO mice are resistant to denervation-induced muscle wasting ${ }^{247}$. However, to date very few studies have investigated the effect of Parkin in additional muscle wasting models. Importantly, chronic inflammation and increased inflammatory response have been demonstrated previously in PD patients ${ }^{349}$. Given that glucocorticoids (GC) are commonly used anti-inflammatory agents that have been shown previously to induce skeletal muscle atrophy ${ }^{350}$, it would be interesting to study GC-induced skeletal muscle atrophy in Parkin KO mice. Dexamethasone is a synthetic glucocorticoid that is widely used in medicine ${ }^{351}$. As such, I would propose to investigate the effect of loss of Parkin on the progression of muscle atrophy associated with excess dexamethasone treatment. To do so, mice will be administered dexamethasone in drinking water for up to 15 days. Subsequent analysis of the resulting skeletal muscle phenotype and the levels of muscle wasting markers will be assessed in in control and Parkin KO mice. This study will allow us to understand if loss of Parkin is able to impart protection against muscle wasting stimuli, in addition to denervation, and would also provide us with an opportunity to further understand, at the molecular level, the role of Parkin in GC-induced skeletal muscle atrophy.

PARKIN is a RING-between-RING type E3 ubiquitin ligase that has been shown to direct specific protein substrates to ubiquitin and facilitate their degradation ${ }^{352}$. PARKIN translocation to mitochondria is regulated by a serine/threonine kinase, PINK1, in a phosphorylation dependent manner. Also, PARKIN has other interacting partners that have been shown to play critical roles in regulating PARKIN function, thereby mitophagy ${ }^{353}$. Hence, it would be interesting to investigate the potential interacting partners of PARKIN in muscle wasting conditions. A recent study has revealed increased levels of Parkin mRNA expression and PARKIN protein levels in denervated muscle ${ }^{354}$, suggesting that Parkin is involved in denervation-induced muscle wasting phenotype in mice. However, the potential partners of PARKIN in denervation model remains to be unstudied. To elucidate the PARKIN-interacting proteins in denervation model, I would cut the sciatic nerve of mice and collect the protein form hind limb muscle 7 days and 14 days post-denervation. Subsequent protein isolation and utilization of a commercial protein-protein interaction microarray chip would provide compelling evidences to portray mechanism behind Parkin-mediated denervation in mouse model. 
Given that: 1) PD is the second most common neurodegenerative disease associated with aging around the world and 2) mitochondrial function is impaired during aging, it would be interesting to study Parkin function during sarcopenia, which is defined as aging-related skeletal muscle wasting. Aging has been shown to impair PARKIN translocation to mitochondria upon stress stimuli, which has been shown to impair mitophagy ${ }^{355}$. Moreover, a recent study has suggested that parkin overexpression protects against proteotoxicity observed in fly muscle due to aging and extends lifespan ${ }^{292}$. As such, I would like to investigate the role of Parkin in aging mice to explore whether Parkin overexpression can protect mice from the detrimental effects of aging-mediated muscle wasting. To investigate the role of Parkin during aging, I would compare the expression of Parkin mRNA and PARKIN protein levels in skeletal muscle of aged (2-year-old) and young mice (6-month-old). To further elucidate a protective role for Parkin during aging, it would be important to investigate the skeletal muscle phenotype in Parkin overexpressing muscle as compared to control. In addition, I would further analyse muscle wasting markers in skeletal muscle tissue of Parkin over expressing muscle, such as the levels of key atrogenes, to investigate the role of Parkin in the progression of muscle wasting associated with aging.

A recent report has revealed that mitophagy is required for successful myogenic differentiation of $\mathrm{C} 2 \mathrm{C} 12$ myoblasts in vitro ${ }^{294}$. In addition, Parkin has been shown to be differentially expressed during developmental stages. Therefore, it would be interesting to study the role of Parkin in myogenesis. First, the levels of Parkin mRNA expression and protein in differentiating $\mathrm{C} 2 \mathrm{C} 12$ muscle cells will be analysed to elucidate a potential role for Parkin during differentiation. Next, the levels of critical myogenic differentiation markers will be assessed, such as MyoD and myogenin, and the levels of these myogenic markers will be correlated to changes in Parkin levels. In addition, histological staining will be performed and myotube number and myotube fusion index will be further quantified in Parkin loss-of-function and gain-of-function models. These data will provide insight into the direct role of Parkin in controlling myogenic differentiation.

Besides mammalian studies, a glance to skeletal muscle and PD from human perspective would reveal mechanisms behind PD and possible therapeutic targets to cure PD. As such, investigation of skeletal muscle from PD patients would provide more realistic and precise evidences to treat the second most common neurodegenerative disease among aging population. To achieve this goal, mitochondrial respiration of 
primary myotubes collected from PD patients need to be assessed using Seahorse XF analyser. Protein levels and activity of mitochondrial OXPHOS complexes need to be assessed to further validate altered mitochondrial respiratory capacity in skeletal muscle of PD patients. Also, mitochondrial fusion and fission proteins should be investigated in protein lysates collected from skeletal muscle of PD patients. Moreover, mitochondrial ROS levels should be measured to understand mechanism further.

Overall, the results presented in this thesis identify an emerging role for Parkin in the regulation of skeletal muscle mass and function. In addition, results from this thesis further suggest that methodologies aimed at maintaining balanced Parkin expression and function may have therapeutic potential for individuals suffering from PD. 


\section{APPENDICES}

Appendix 6.1: Copyright clearance obtained for Figure 1.3

Copyright

Clearance

Center

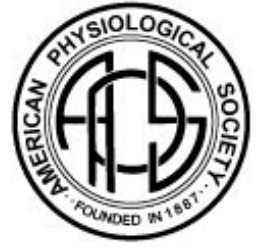

Title: $\quad$ Transforming growth factor- $\beta$ and myostatin signaling in skeletal muscle

Author: Helen D. Kollias,John C.

McDermott

Logged in as:

Nesibe Peker

Nanyang Technological

University

Account $=$ :

3001101140

Publication: Journal of Applied Physiology

Publisher: The American Physiological

Date: $\quad$ Mar 1, 2008

Copyright (c) 2008, Copyright (5) 2008 the American

Physiological Society

Permission Not Required

Permission is not required for this type of use.

\section{BACK}


Appendix 6.2: Copyright clearance obtained for Figure 1.5

\section{Customer Information}

Customer: Nesibe Peker

Account Number: 3001101140

Organization: Nanyang Technological

University

Email: nesibe001@e.ntu.edu.sg

Phone: +65 94669588

Search order details by: Choose One

This is not an invoice

Order Details

Journal of the American Society of Nephrology : JASN

Billing Status:

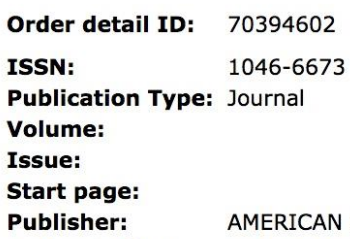


Appendix 6.3: Copyright clearance obtained for Figure 1.7

RightsLink Printable License

$22 / 3 / 17,11: 40$ PM

NATURE PUBLISHING GROUP LICENSE

TERMS AND CONDITIONS

Mar 22, 2017

This Agreement between Nanyang Technological University -- Nesibe Peker ("You") and Nature Publishing Group ("Nature Publishing Group") consists of your license details and the terms and conditions provided by Nature Publishing Group and Copyright Clearance Center.

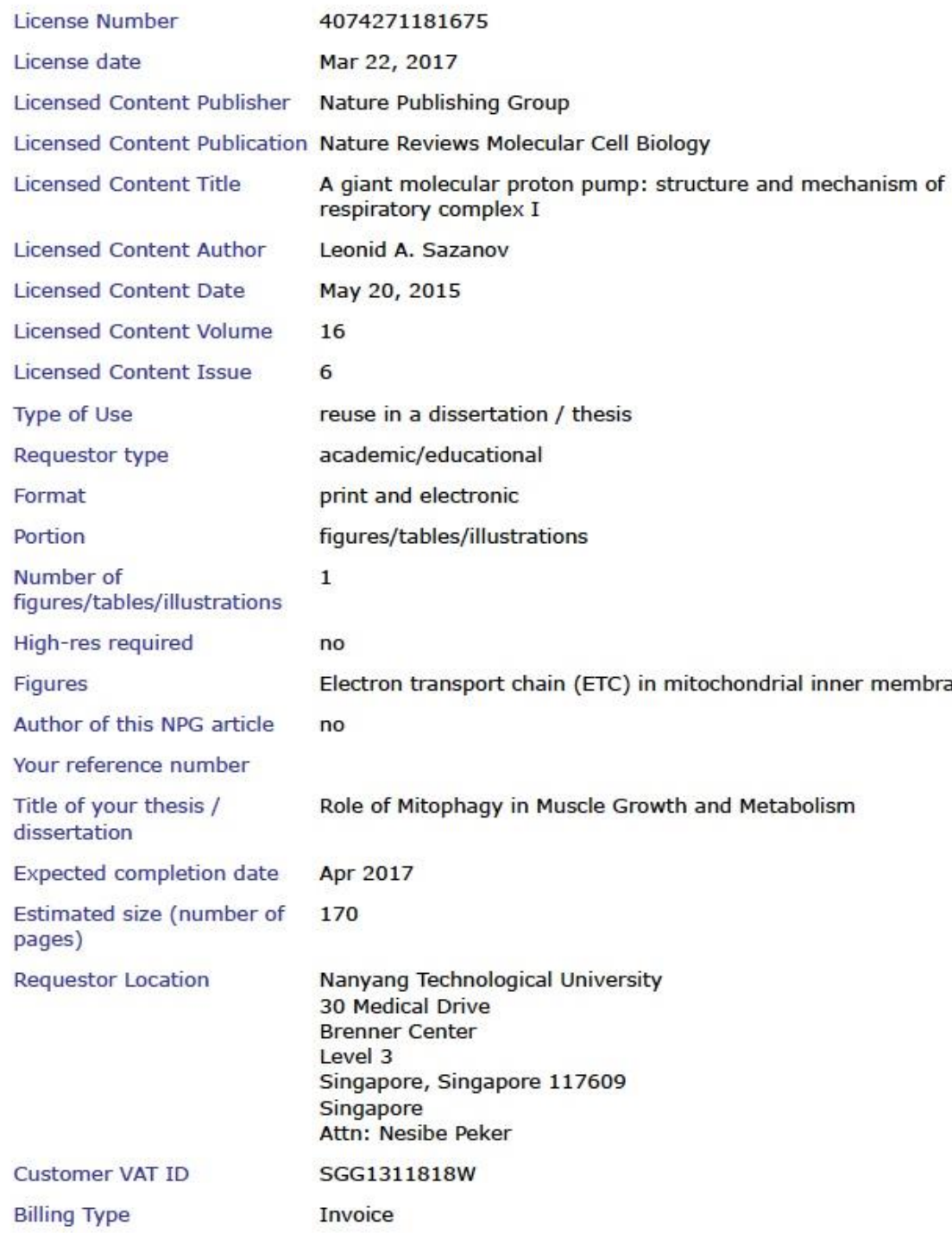


Appendix 6.4: Copyright clearance obtained for Figure 1.8

\section{(ㄷ)(1)}

\section{Attribution 4.0 International (CC BY 4.0)}

This is a humen-resdable summery of (and not a substitute for) the license. Discleimer.

\section{You are free to:}

Share - copy and redistribute the material in any medium or format

Adapt - remix, transform, and build upon the material

for any purpose, even commercially.

The licersor cennot revoke these freedoma ss long ss you follow the

license terms.

\section{Under the following terms:}

Attribution - You must give appropriate credit, provide a

link to the license, and indicate if changes were made. You

may do so in any reasonable manner, but not in any way that suggests the licensor endorses you or your use.

No additional restrictions - You may not apply legal terms or technological measures that legally restrict others from

doing anything the license permits.

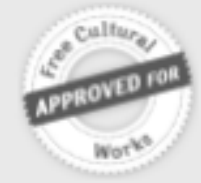


Appendix 6.5: Copyright clearance obtained for Figure 1.9

RightsLink Printable License

NATURE PUBLISHING GROUP LICENSE TERMS AND CONDITIONS

This Agreement between Nanyang Technological University -- Nesibe Peker ("You") and Nature Publishing Group ("Nature Publishing Group") consists of your license details and the terms and conditions provided by Nature Publishing Group and Copyright Clearance Center.

\begin{tabular}{|c|c|}
\hline License Number & 4074280171050 \\
\hline License date & Mar 22, 2017 \\
\hline Licensed Content Publisher & Nature Publishing Group \\
\hline Licensed Content Publication & Nature Reviews Drug Discovery \\
\hline Licensed Content Title & Pharmacological approaches to restore mitochondrial function \\
\hline Licensed Content Author & Pénélope A. Andreux, Riekelt H. Houtkooper, Johan Auwerx \\
\hline Licensed Content Date & May 13, 2013 \\
\hline Licensed Content Volume & 12 \\
\hline Licensed Content Issue & 6 \\
\hline Type of Use & reuse in a dissertation / thesis \\
\hline Requestor type & academic/educational \\
\hline Format & print and electronic \\
\hline Portion & figures/tables/illustrations \\
\hline $\begin{array}{l}\text { Number of } \\
\text { figures/tables/illustrations }\end{array}$ & 1 \\
\hline High-res required & no \\
\hline Figures & Figure 2: Mitochondrial quality control processes \\
\hline Author of this NPG article & no \\
\hline \multicolumn{2}{|l|}{ Your reference number } \\
\hline $\begin{array}{l}\text { Title of your thesis / } \\
\text { dissertation }\end{array}$ & Role of Mitophagy in Muscle Growth and Metabolism \\
\hline Expected completion date & Apr 2017 \\
\hline $\begin{array}{l}\text { Estimated size (number of } \\
\text { pages) }\end{array}$ & 170 \\
\hline Requestor Location & $\begin{array}{l}\text { Nanyang Technological University } \\
30 \text { Medical Drive } \\
\text { Brenner Center } \\
\text { Level } 3 \\
\text { Singapore, Singapore } 117609 \\
\text { Singapore } \\
\text { Attn: Nesibe Peker }\end{array}$ \\
\hline Customer VAT ID & SGG1311818W \\
\hline Billing Type & Invoice \\
\hline
\end{tabular}


Appendix 6.6: Copyright clearance obtained for Figure 1.10

RightsLink Printable License

CAMBRIDGE UNIVERSITY PRESS LICENSE TERMS AND CONDITIONS

This Agreement between Nanyang Technological University -- Nesibe Peker ("You") and Cambridge University Press ("Cambridge University Press") consists of your license details and the terms and conditions provided by Cambridge University Press and Copyright Clearance Center.

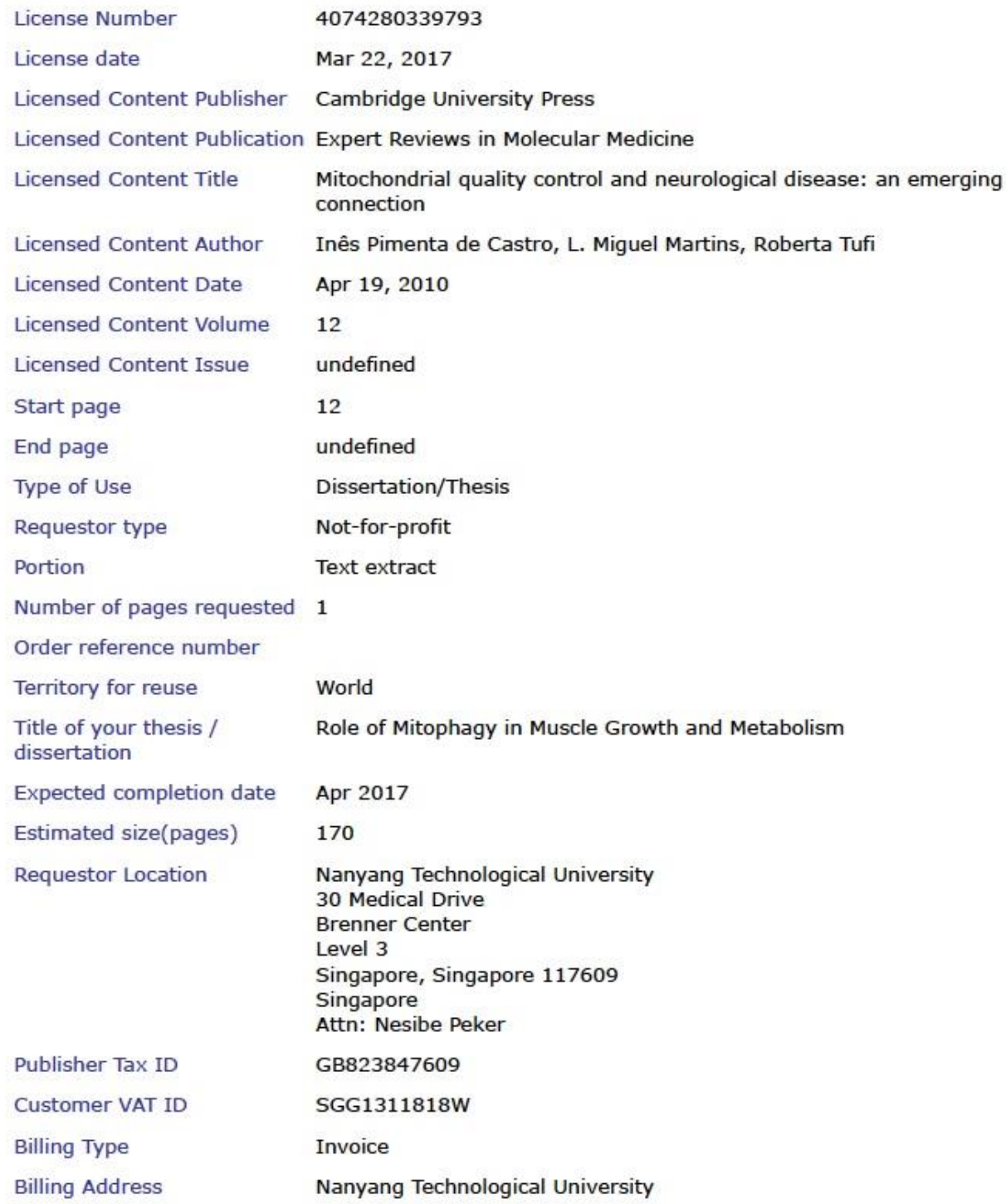


Appendix 6.7: Copyright clearance obtained for Figure 2.1

Copyright

Clearance

Center

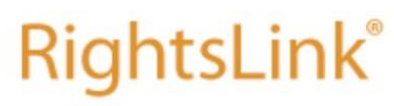

$\begin{array}{cl}\text { Title: } & \begin{array}{l}\text { Oxidative stress induces } \\ \text { mitochondrial dysfunction in a } \\ \text { subset of autistic lymphoblastoid } \\ \text { cell lines } \\ \begin{array}{c}\text { publishing } \\ \text { group }\end{array}\end{array} \\ & \begin{array}{l}\text { Author: } \\ \text { S Rose, R E Frye, J Slattery, R } \\ \text { Wynne, M Tippett et al. } \\ \text { Publication: Translational Psychiatry } \\ \text { Date: } \quad \text { Nature Publishing Group } \\ \text { Copyright @ 2014, Rights Managed by Nature } \\ \text { Publishing Group }\end{array}\end{array}$

Logged in as:

Nesibe Peker

Nanyang Technological

University

Account \#:

3001101140

LOGOUT

\section{Creative Commons}

The request you have made is considered to be non-commercial/educational. As the article you have requested has been distributed under a Creative Commons license (Attribution-Noncommercial), you may reuse this material for non-commercial/educational purposes without obtaining additional permission from Nature Publishing Group, providing that the author and the original source of publication are fully acknowledged(please see the article itself for the license version number). You may reuse this material without obtaining permission from Nature Publishing Group, providing that the author and the original source of publication are fully acknowledged, as per the terms of the license. For license terms, please see http://creativecommons.org/

\section{BACK}

Copyright (c) 2017 Copyright Clearance Center, Inc. All Rights Reserved. Privacy statement. Terms and Conditions. Comments? We would like to hear from you. E-mail us at customercare@copyright.com 


\section{REFERENCES}

1. Crescenzo, R., et al., Skeletal muscle mitochondrial energetic efficiency and aging. Int J Mol Sci, 2015. 16(5): p. 10674-85.

2. Lodish H, B.A., Zipursky SL, Matsudaira P, Baltimore D, Darnell J, Muscle: A Specialized Contractile Machine, in Molecular Cell Biology. 2000, W.H. Freeman and Company,: New York. p. xxxix, 1084 p. ill. (some col.) + 1 CDROM (4 3/4 in.).

3. Frontera, W.R. and J. Ochala, Skeletal muscle: a brief review of structure and function. Calcif Tissue Int, 2015. 96(3): p. 183-95.

4. Huxley, A.F., Muscle structure and theories of contraction. Prog Biophys Biophys Chem, 1957. 7: p. 255-318.

5. Sheetz, M.P. and J.A. Spudich, Movement of myosin-coated fluorescent beads on actin cables in vitro. Nature, 1983. 303(5912): p. 31-5.

6. Tajbakhsh, S., Skeletal muscle stem cells in developmental versus regenerative myogenesis. J Intern Med, 2009. 266(4): p. 372-89.

7. Hofmann, M., et al., WNT signaling, in synergy with T/TBX6, controls Notch signaling by regulating Dll1 expression in the presomitic mesoderm of mouse embryos. Genes Dev, 2004. 18(22): p. 2712-7.

8. Aulehla, A. and O. Pourquie, Signaling gradients during paraxial mesoderm development. Cold Spring Harb Perspect Biol, 2010. 2(2): p. a000869.

9. Hammond, C.L., et al., Signals and myogenic regulatory factors restrict pax3 and pax7 expression to dermomyotome-like tissue in zebrafish. Dev Biol, 2007. 302(2): p. 504-21.

10. Goulding, M.D., et al., Pax-3, a novel murine DNA binding protein expressed during early neurogenesis. EMBO J, 1991. 10(5): p. 1135-47.

11. Kiefer, J.C. and S.D. Hauschka, Myf-5 is transiently expressed in nonmuscle mesoderm and exhibits dynamic regional changes within the presegmented mesoderm and somites I-IV. Dev Biol, 2001. 232(1): p. 77-90.

12. Seale, P., et al., Pax7 is required for the specification of myogenic satellite cells. Cell, 2000. 102(6): p. 777-86.

13. Pownall, M.E., M.K. Gustafsson, and C.P. Emerson, Jr., Myogenic regulatory factors and the specification of muscle progenitors in vertebrate embryos. Annu Rev Cell Dev Biol, 2002. 18: p. 747-83.

14. Buckingham, M., et al., The formation of skeletal muscle: from somite to limb. $\mathbf{J}$ Anat, 2003. 202(1): p. 59-68. 
15. Yin, H., F. Price, and M.A. Rudnicki, Satellite cells and the muscle stem cell niche. Physiol Rev, 2013. 93(1): p. 23-67.

16. Allen, R.E. and L.K. Boxhorn, Regulation of skeletal muscle satellite cell proliferation and differentiation by transforming growth factor-beta, insulin-like growth factor I, and fibroblast growth factor. J Cell Physiol, 1989. 138(2): p. 311-5.

17. Allen, R.E. and L.L. Rankin, Regulation of satellite cells during skeletal muscle growth and development. Proc Soc Exp Biol Med, 1990. 194(2): p. 81-6.

18. Wozniak, A.C. and J.E. Anderson, Nitric oxide-dependence of satellite stem cell activation and quiescence on normal skeletal muscle fibers. Dev Dyn, 2007. 236(1): p. 240-50.

19. Pisconti, A., et al., Follistatin induction by nitric oxide through cyclic GMP: a tightly regulated signaling pathway that controls myoblast fusion. J Cell Biol, 2006. 172(2): p. 233-44.

20. Conboy, M.J., A.O. Karasov, and T.A. Rando, High incidence of non-random template strand segregation and asymmetric fate determination in dividing stem cells and their progeny. PLoS Biol, 2007. 5(5): p. e102.

21. Olguin, H.C. and B.B. Olwin, Pax-7 up-regulation inhibits myogenesis and cell cycle progression in satellite cells: a potential mechanism for self-renewal. Dev Biol, 2004. 275(2): p. 375-88.

22. Olguin, H.C., et al., Reciprocal inhibition between Pax7 and muscle regulatory factors modulates myogenic cell fate determination. J Cell Biol, 2007. 177(5): p. 769-79.

23. Shefer, G., M. Wleklinski-Lee, and Z. Yablonka-Reuveni, Skeletal muscle satellite cells can spontaneously enter an alternative mesenchymal pathway. $\mathrm{J}$ Cell Sci, 2004. 117(Pt 22): p. 5393-404.

24. Christov, C., et al., Muscle satellite cells and endothelial cells: close neighbors and privileged partners. Mol Biol Cell, 2007. 18(4): p. 1397-409.

25. Zammit, P.S., et al., Muscle satellite cells adopt divergent fates: a mechanism for self-renewal? J Cell Biol, 2004. 166(3): p. 347-57.

26. Nagata, Y., et al., Entry of muscle satellite cells into the cell cycle requires sphingolipid signaling. J Cell Biol, 2006. 174(2): p. 245-53.

27. Arnold, L., et al., Inflammatory monocytes recruited after skeletal muscle injury switch into antiinflammatory macrophages to support myogenesis. J Exp Med, 2007. 204(5): p. 1057-69.

28. Darby, I.A., et al., The myofibroblast, a key cell in normal and pathological tissue repair. Cell Mol Life Sci, 2016. 73(6): p. 1145-57. 
29. Zierath, J.R. and J.A. Hawley, Skeletal muscle fiber type: influence on contractile and metabolic properties. PLoS Biol, 2004. 2(10): p. e348.

30. Needham, D.M., A Quantitative Study of Succinic Acid in Muscle. II: The Metabolic Relationships of Succinic, Malic and Fumaric Acids. Biochem J, 1927. 21(3): p. 739-50.

31. Wachstein, M. and E. Meisel, The distribution of histochemically demonstrable succinic dehydrogenase and of mitochondria in tongue and skeletal muscles. $\mathrm{J}$ Biophys Biochem Cytol, 1955. 1(6): p. 483-8.

32. Brooke, M.H. and K.K. Kaiser, Three human myosin ATPase systems and their importance in muscle pathology. Neurology, 1970. 20(4): p. 404-5.

33. Wilson, J.M., et al., The effects of endurance, strength, and power training on muscle fiber type shifting. J Strength Cond Res, 2012. 26(6): p. 1724-9.

34. Karp, G., The Role of Anaerobic and Aerobic Metabolism in Excercise, in Cell and Molecular Biology: Concepts and Experiments. 2009, John Wiley \& Sons.

35. Vandenburgh, H., et al., Space travel directly induces skeletal muscle atrophy. FASEB J, 1999. 13(9): p. 1031-8.

36. Phillips, S.M., E.I. Glover, and M.J. Rennie, Alterations of protein turnover underlying disuse atrophy in human skeletal muscle. J Appl Physiol (1985), 2009. 107(3): p. 645-54.

37. Castro, M.J., et al., Influence of complete spinal cord injury on skeletal muscle mechanics within the first 6 months of injury. Eur J Appl Physiol, 2000. 81(12): p. 128-31.

38. Booth, F.W. and M.J. Seider, Early change in skeletal muscle protein synthesis after limb immobilization of rats. J Appl Physiol Respir Environ Exerc Physiol, 1979. 47(5): p. 974-7.

39. de Boer, M.D., et al., Time course of muscular, neural and tendinous adaptations to 23 day unilateral lower-limb suspension in young men. J Physiol, 2007. 583(Pt 3): p. 1079-91.

40. Purves, D. and S.M. Williams, The Lower Motor Neuron Syndrome, in Neuroscience. 2001, Sinauer Associates: Sunderland, Mass. p. xviii, 681, 16, 3, $25 \mathrm{p}$.

41. Rosenberg, I.H., Sarcopenia: origins and clinical relevance. Clin Geriatr Med, 2011. 27(3): p. 337-9.

42. Balagopal, P., et al., Effects of aging on in vivo synthesis of skeletal muscle myosin heavy-chain and sarcoplasmic protein in humans. Am J Physiol, 1997. 273(4 Pt 1): p. E790-800. 
43. Larsson, L., Morphological and functional characteristics of the ageing skeletal muscle in man. A cross-sectional study. Acta Physiol Scand Suppl, 1978. 457: p. 1-36.

44. Lexell, J., C.C. Taylor, and M. Sjostrom, What is the cause of the ageing atrophy? Total number, size and proportion of different fiber types studied in whole vastus lateralis muscle from 15- to 83-year-old men. J Neurol Sci, 1988. 84(2-3): p. 275-94.

45. Ciciliot, S., et al., Muscle type and fiber type specificity in muscle wasting. Int J Biochem Cell Biol, 2013. 45(10): p. 2191-9.

46. Andersen, J.L., Muscle fibre type adaptation in the elderly human muscle. Scand J Med Sci Sports, 2003. 13(1): p. 40-7.

47. Lok, C., Cachexia: The last illness. Nature, 2015. 528(7581): p. 182-3.

48. Aoyagi, T., et al., Cancer cachexia, mechanism and treatment. World J Gastrointest Oncol, 2015. 7(4): p. 17-29.

49. Bowen, T.S., G. Schuler, and V. Adams, Skeletal muscle wasting in cachexia and sarcopenia: molecular pathophysiology and impact of exercise training. $\mathrm{J}$ Cachexia Sarcopenia Muscle, 2015. 6(3): p. 197-207.

50. Argiles, J.M., et al., Cancer cachexia: understanding the molecular basis. Nat Rev Cancer, 2014. 14(11): p. 754-62.

51. Argiles, J.M., et al., The role of cytokines in cancer cachexia. Curr Opin Support Palliat Care, 2009. 3(4): p. 263-8.

52. Tisdale, M.J., Biology of cachexia. J Natl Cancer Inst, 1997. 89(23): p. 1763-73.

53. Frost, R.A., et al., Hormone, cytokine, and nutritional regulation of sepsisinduced increases in atrogin-1 and MuRF1 in skeletal muscle. Am J Physiol Endocrinol Metab, 2007. 292(2): p. E501-12.

54. Li, Y.P., et al., TNF-alpha acts via p38 MAPK to stimulate expression of the ubiquitin ligase atrogin1/MAFbx in skeletal muscle. FASEB J, 2005. 19(3): p. 362-70.

55. Nowak, K.J. and K.E. Davies, Duchenne muscular dystrophy and dystrophin: pathogenesis and opportunities for treatment. EMBO Rep, 2004. 5(9): p. 872-6.

56. Yiu, E.M. and A.J. Kornberg, Duchenne muscular dystrophy. Neurol India, 2008. 56(3): p. 236-47.

57. Parent, A., Duchenne De Boulogne: a pioneer in neurology and medical photography. Can J Neurol Sci, 2005. 32(3): p. 369-77.

58. Davidson, Z.E. and H. Truby, A review of nutrition in Duchenne muscular dystrophy. J Hum Nutr Diet, 2009. 22(5): p. 383-93. 
59. Straub, V. and K.P. Campbell, Muscular dystrophies and the dystrophinglycoprotein complex. Curr Opin Neurol, 1997. 10(2): p. 168-75.

60. Cohn, R.D. and K.P. Campbell, Molecular basis of muscular dystrophies. Muscle Nerve, 2000. 23(10): p. 1456-71.

61. Tidball, J.G. and S.A. Villalta, NO may prompt calcium leakage in dystrophic muscle. Nat Med, 2009. 15(3): p. 243-4.

62. Sartorelli, V. and M. Fulco, Molecular and cellular determinants of skeletal muscle atrophy and hypertrophy. Sci STKE, 2004. 2004(244): p. re11.

63. Millward, D.J., et al., Relationship between protein synthesis and RNA content in skeletal muscle. Nature, 1973. 241(5386): p. 204-5.

64. Nandagopal, N. and P.P. Roux, Regulation of global and specific $m R N A$ translation by the mTOR signaling pathway. Translation (Austin), 2015. 3(1): p. e983402.

65. Nader, G.A., T.J. McLoughlin, and K.A. Esser, mTOR function in skeletal muscle hypertrophy: increased ribosomal RNA via cell cycle regulators. Am J Physiol Cell Physiol, 2005. 289(6): p. C1457-65.

66. Bodine, S.C., et al., Identification of ubiquitin ligases required for skeletal muscle atrophy. Science, 2001. 294(5547): p. 1704-8.

67. O'Neil, T.K., et al., The role of phosphoinositide 3-kinase and phosphatidic acid in the regulation of mammalian target of rapamycin following eccentric contractions. J Physiol, 2009. 587(Pt 14): p. 3691-701.

68. Carroll, M., J. Dyer, and W.S. Sossin, Serotonin increases phosphorylation of synaptic 4EBP through TOR, but eukaryotic initiation factor $4 E$ levels do not limit somatic cap-dependent translation in aplysia neurons. Mol Cell Biol, 2006. 26(22): p. 8586-98.

69. Goodman, C.A., D.L. Mayhew, and T.A. Hornberger, Recent progress toward understanding the molecular mechanisms that regulate skeletal muscle mass. Cell Signal, 2011. 23(12): p. 1896-906.

70. Armstrong, D.D. and K.A. Esser, Wnt/beta-catenin signaling activates growthcontrol genes during overload-induced skeletal muscle hypertrophy. Am J Physiol Cell Physiol, 2005. 289(4): p. C853-9.

71. Zeller, K.I., et al., Global mapping of $c$-Myc binding sites and target gene networks in human B cells. Proc Natl Acad Sci U S A, 2006. 103(47): p. 178349.

72. Goll, D.E., et al., The calpain system. Physiol Rev, 2003. 83(3): p. 731-801.

73. Taveau, M., et al., Calpain 3 is activated through autolysis within the active site and lyses sarcomeric and sarcolemmal components. Mol Cell Biol, 2003. 23(24): p. 9127-35. 
74. Hong, D.H. and N.E. Forsberg, Effects of dexamethasone on protein degradation and protease gene expression in rat L8 myotube cultures. Mol Cell Endocrinol, 1995. 108(1-2): p. 199-209.

75. Tang, H., et al., Identification and characterization of differentially expressed genes in denervated muscle. Mol Cell Neurosci, 2000. 16(2): p. 127-40.

76. Voisin, L., et al., Muscle wasting in a rat model of long-lasting sepsis results from the activation of lysosomal, Ca2+-activated, and ubiquitin-proteasome proteolytic pathways. J Clin Invest, 1996. 97(7): p. 1610-7.

77. Bullard, B., G. Sainsbury, and N. Miller, Digestion of proteins associated with the Z-disc by calpain. J Muscle Res Cell Motil, 1990. 11(3): p. 271-9.

78. Bartoli, M. and I. Richard, Calpains in muscle wasting. Int J Biochem Cell Biol, 2005. 37(10): p. 2115-33.

79. Kirschke, H. and B. Wiederanders, Lysosomal proteinases. Acta Histochem, 1987. 82(1): p. 2-4.

80. Deval, C., et al., Identification of cathepsin $L$ as a differentially expressed message associated with skeletal muscle wasting. Biochem J, 2001. 360(Pt 1): p. $143-50$.

81. Furuno, K. and A.L. Goldberg, The activation of protein degradation in muscle by Ca2+ or muscle injury does not involve a lysosomal mechanism. Biochem $\mathrm{J}$, 1986. 237(3): p. 859-64.

82. Taillandier, D., et al., Coordinate activation of lysosomal, Ca 2+-activated and ATP-ubiquitin-dependent proteinases in the unweighted rat soleus muscle. Biochem J, 1996. 316 ( Pt 1): p. 65-72.

83. Mayer, R.J., The meteoric rise of regulated intracellular proteolysis. Nat Rev Mol Cell Biol, 2000. 1(2): p. 145-8.

84. Jackman, R.W. and S.C. Kandarian, The molecular basis of skeletal muscle atrophy. Am J Physiol Cell Physiol, 2004. 287(4): p. C834-43.

85. Haas, A.L., et al., Ubiquitin-activating enzyme. Mechanism and role in proteinubiquitin conjugation. J Biol Chem, 1982. 257(5): p. 2543-8.

86. Finley, D., Recognition and processing of ubiquitin-protein conjugates by the proteasome. Annu Rev Biochem, 2009. 78: p. 477-513.

87. Vilchez, D., I. Saez, and A. Dillin, The role of protein clearance mechanisms in organismal ageing and age-related diseases. Nat Commun, 2014. 5: p. 5659.

88. Scheffner, M., U. Nuber, and J.M. Huibregtse, Protein ubiquitination involving an E1-E2-E3 enzyme ubiquitin thioester cascade. Nature, 1995. 373(6509): p. 81-3. 
89. Jackson, P.K., et al., The lore of the RINGs: substrate recognition and catalysis by ubiquitin ligases. Trends Cell Biol, 2000. 10(10): p. 429-39.

90. Hatakeyama, S. and K.I. Nakayama, U-box proteins as a new family of ubiquitin ligases. Biochem Biophys Res Commun, 2003. 302(4): p. 635-45.

91. Wing, S.S., Control of ubiquitination in skeletal muscle wasting. Int J Biochem Cell Biol, 2005. 37(10): p. 2075-87.

92. Sacheck, J.M., et al., Rapid disuse and denervation atrophy involve transcriptional changes similar to those of muscle wasting during systemic diseases. FASEB J, 2007. 21(1): p. 140-55.

93. Gomes, M.D., et al., Atrogin-1, a muscle-specific F-box protein highly expressed during muscle atrophy. Proc Natl Acad Sci U S A, 2001. 98(25): p. 14440-5.

94. Baehr, L.M., J.D. Furlow, and S.C. Bodine, Muscle sparing in muscle RING finger 1 null mice: response to synthetic glucocorticoids. J Physiol, 2011. 589(Pt 19): p. 4759-76.

95. Cong, H., et al., Inhibition of atrogin-1/MAFbx expression by adenovirusdelivered small hairpin RNAs attenuates muscle atrophy in fasting mice. Hum Gene Ther, 2011. 22(3): p. 313-24.

96. Csibi, A., et al., The translation regulatory subunit eIF3f controls the kinasedependent mTOR signaling required for muscle differentiation and hypertrophy in mouse. PLoS One, 2010. 5(2): p. e8994.

97. Tintignac, L.A., et al., Degradation of MyoD mediated by the SCF (MAFbx) ubiquitin ligase. J Biol Chem, 2005. 280(4): p. 2847-56.

98. Clarke, B.A., et al., The E3 Ligase MuRF1 degrades myosin heavy chain protein in dexamethasone-treated skeletal muscle. Cell Metab, 2007. 6(5): p. 376-85.

99. Cohen, S., et al., During muscle atrophy, thick, but not thin, filament components are degraded by MuRF1-dependent ubiquitylation. J Cell Biol, 2009. 185(6): p. 1083-95.

100. Kedar, V., et al., Muscle-specific RING finger 1 is a bona fide ubiquitin ligase that degrades cardiac troponin I. Proc Natl Acad Sci U S A, 2004. 101(52): p. 18135-40.

101. Bence, N.F., R.M. Sampat, and R.R. Kopito, Impairment of the ubiquitinproteasome system by protein aggregation. Science, 2001. 292(5521): p. 15525.

102. Saftig, P., W. Beertsen, and E.L. Eskelinen, LAMP-2: a control step for phagosome and autophagosome maturation. Autophagy, 2008. 4(4): p. 510-2.

103. Trinh, J. and M. Farrer, Advances in the genetics of Parkinson disease. Nat Rev Neurol, 2013. 9(8): p. 445-54. 
104. Oz-Levi, D., et al., Mutation in TECPR2 reveals a role for autophagy in hereditary spastic paraparesis. Am J Hum Genet, 2012. 91(6): p. 1065-72.

105. Cullup, T., et al., Recessive mutations in EPG5 cause Vici syndrome, a multisystem disorder with defective autophagy. Nat Genet, 2013. 45(1): p. 83-7.

106. Kundu, M. and C.B. Thompson, Macroautophagy versus mitochondrial autophagy: a question of fate? Cell Death Differ, 2005. 12 Suppl 2: p. 1484-9.

107. Glick, D., S. Barth, and K.F. Macleod, Autophagy: cellular and molecular mechanisms. J Pathol, 2010. 221(1): p. 3-12.

108. Kabeya, Y., et al., Atg17 functions in cooperation with Atgl and Atg13 in yeast autophagy. Mol Biol Cell, 2005. 16(5): p. 2544-53.

109. Diaz-Troya, S., et al., The role of TOR in autophagy regulation from yeast to plants and mammals. Autophagy, 2008. 4(7): p. 851-65.

110. Raben, N., et al., Suppression of autophagy in skeletal muscle uncovers the accumulation of ubiquitinated proteins and their potential role in muscle damage in Pompe disease. Hum Mol Genet, 2008. 17(24): p. 3897-908.

111. Barth, S., D. Glick, and K.F. Macleod, Autophagy: assays and artifacts. J Pathol, 2010. 221(2): p. 117-24.

112. Pankiv, S., et al., p62/SQSTM1 binds directly to Atg8/LC3 to facilitate degradation of ubiquitinated protein aggregates by autophagy. $\mathrm{J}$ Biol Chem, 2007. 282(33): p. 24131-45.

113. Narendra, D., et al., p62/SQSTM1 is required for Parkin-induced mitochondrial clustering but not mitophagy; VDACl is dispensable for both. Autophagy, 2010. 6(8): p. 1090-106.

114. Grumati, P., et al., Autophagy is defective in collagen VI muscular dystrophies, and its reactivation rescues myofiber degeneration. Nat Med, 2010. 16(11): p. 1313-20.

115. O'Leary, M.F., et al., Denervation-induced mitochondrial dysfunction and autophagy in skeletal muscle of apoptosis-deficient animals. Am J Physiol Cell Physiol, 2012. 303(4): p. C447-54.

116. Brocca, L., et al., The time course of the adaptations of human muscle proteome to bed rest and the underlying mechanisms. J Physiol, 2012. 590(20): p. 521130 .

117. Penna, F., et al., Autophagic degradation contributes to muscle wasting in cancer cachexia. Am J Pathol, 2013. 182(4): p. 1367-78.

118. Wohlgemuth, S.E., et al., Skeletal muscle autophagy and apoptosis during aging: effects of calorie restriction and life-long exercise. Exp Gerontol, 2010. 45(2): p. 138-48. 
119. Mammucari, C., et al., FoxO3 controls autophagy in skeletal muscle in vivo. Cell Metab, 2007. 6(6): p. 458-71.

120. Musaro, A., et al., Localized Igf-1 transgene expression sustains hypertrophy and regeneration in senescent skeletal muscle. Nat Genet, 2001. 27(2): p. 195200.

121. Calnan, D.R. and A. Brunet, The FoxO code. Oncogene, 2008. 27(16): p. 227688 .

122. Manning, B.D. and L.C. Cantley, AKT/PKB signaling: navigating downstream. Cell, 2007. 129(7): p. 1261-74.

123. Lai, K.M., et al., Conditional activation of akt in adult skeletal muscle induces rapid hypertrophy. Mol Cell Biol, 2004. 24(21): p. 9295-304.

124. Barnes, P.J., Anti-inflammatory actions of glucocorticoids: molecular mechanisms. Clin Sci (Lond), 1998. 94(6): p. 557-72.

125. Hasselgren, P.O., Glucocorticoids and muscle catabolism. Curr Opin Clin Nutr Metab Care, 1999. 2(3): p. 201-5.

126. Lecker, S.H., et al., Multiple types of skeletal muscle atrophy involve a common program of changes in gene expression. FASEB J, 2004. 18(1): p. 39-51.

127. Gupta, A. and Y. Gupta, Glucocorticoid-induced myopathy: Pathophysiology, diagnosis, and treatment. Indian J Endocrinol Metab, 2013. 17(5): p. 913-6.

128. Goldberg, A.L., et al., Hormonal regulation of protein degradation and synthesis in skeletal muscle. Fed Proc, 1980. 39(1): p. 31-6.

129. Waddell, D.S., et al., The glucocorticoid receptor and FOXO1 synergistically activate the skeletal muscle atrophy-associated MuRF1 gene. Am J Physiol Endocrinol Metab, 2008. 295(4): p. E785-97.

130. Tsuchida, K., Activins, myostatin and related TGF-beta family members as novel therapeutic targets for endocrine, metabolic and immune disorders. Curr Drug Targets Immune Endocr Metabol Disord, 2004. 4(2): p. 157-66.

131. Elkina, Y., et al., The role of myostatin in muscle wasting: an overview. J Cachexia Sarcopenia Muscle, 2011. 2(3): p. 143-151.

132. Wrana, J.L., et al., Mechanism of activation of the TGF-beta receptor. Nature, 1994. 370(6488): p. 341-7.

133. Zhu, X., et al., Myostatin signaling through Smad2, Smad3 and Smad4 is regulated by the inhibitory Smad7 by a negative feedback mechanism. Cytokine, 2004. 26(6): p. 262-72.

134. Langley, B., et al., Myostatin inhibits myoblast differentiation by downregulating MyoD expression. J Biol Chem, 2002. 277(51): p. 49831-40. 
135. Thomas, M., et al., Myostatin, a negative regulator of muscle growth, functions by inhibiting myoblast proliferation. J Biol Chem, 2000. 275(51): p. 40235-43.

136. McPherron, A.C., A.M. Lawler, and S.J. Lee, Regulation of skeletal muscle mass in mice by a new TGF-beta superfamily member. Nature, 1997. 387(6628): p. 83-90.

137. Lee, S.J., et al., Regulation of muscle growth by multiple ligands signaling through activin type II receptors. Proc Natl Acad Sci U S A, 2005. 102(50): p. 18117-22.

138. McFarlane, C., et al., Myostatin induces cachexia by activating the ubiquitin proteolytic system through an NF-kappaB-independent, FoxO1-dependent mechanism. J Cell Physiol, 2006. 209(2): p. 501-14.

139. Trendelenburg, A.U., et al., Myostatin reduces Akt/TORC1/p70S6K signaling, inhibiting myoblast differentiation and myotube size. Am J Physiol Cell Physiol, 2009. 296(6): p. C1258-70.

140. Gallot, Y.S., et al., Myostatin gene inactivation prevents skeletal muscle wasting in cancer. Cancer Res, 2014. 74(24): p. 7344-56.

141. Sagan, L., On the origin of mitosing cells. J Theor Biol, 1967. 14(3): p. 255-74.

142. Zimorski, V., et al., Endosymbiotic theory for organelle origins. Curr Opin Microbiol, 2014. 22: p. 38-48.

143. Sherratt, H.S., Mitochondria: structure and function. Rev Neurol (Paris), 1991. 147(6-7): p. 417-30.

144. Holt, I.J. and A. Reyes, Human mitochondrial DNA replication. Cold Spring Harb Perspect Biol, 2012. 4(12).

145. Turner, P.R., et al., Increased calcium influx in dystrophic muscle. J Cell Biol, 1991. 115(6): p. 1701-12.

146. Sazanov, L.A., A giant molecular proton pump: structure and mechanism of respiratory complex I. Nat Rev Mol Cell Biol, 2015. 16(6): p. 375-88.

147. Mitchell, P., Coupling of phosphorylation to electron and hydrogen transfer by a chemi-osmotic type of mechanism. Nature, 1961. 191: p. 144-8.

148. Alberts, B., The Mitochondrion, in Molecular Biology of the Cell. 2002, Garland Science: New York.

149. Cohen, G. and R.E. Heikkila, The generation of hydrogen peroxide, superoxide radical, and hydroxyl radical by 6-hydroxydopamine, dialuric acid, and related cytotoxic agents. J Biol Chem, 1974. 249(8): p. 2447-52.

150. Zhang, B.T., L.X. Zhao, and J.M. Lin, Study on superoxide and hydroxyl radicals generated in indirect electrochemical oxidation by chemiluminescence and UV-Visible spectra. J Environ Sci (China), 2008. 20(8): p. 1006-11. 
151. Blazquez-Castro, A., T. Breitenbach, and P.R. Ogilby, Singlet oxygen and ROS in a new light: low-dose subcellular photodynamic treatment enhances proliferation at the single cell level. Photochem Photobiol Sci, 2014. 13(9): p. 1235-40.

152. Zorov, D.B., M. Juhaszova, and S.J. Sollott, Mitochondrial reactive oxygen species (ROS) and ROS-induced ROS release. Physiol Rev, 2014. 94(3): p. 90950.

153. Weisiger, R.A. and I. Fridovich, Superoxide dismutase. Organelle specificity. J Biol Chem, 1973. 248(10): p. 3582-92.

154. Sabharwal, S.S. and P.T. Schumacker, Mitochondrial ROS in cancer: initiators, amplifiers or an Achilles' heel? Nat Rev Cancer, 2014. 14(11): p. 709-21.

155. Eto, Y., et al., Succinate-dependent lipid peroxidation and its prevention by reduced ubiquinone in beef heart submitochondrial particles. Arch Biochem Biophys, 1992. 295(1): p. 101-6.

156. Beyer, R.E., The participation of coenzyme $Q$ in free radical production and antioxidation. Free Radic Biol Med, 1990. 8(6): p. 545-65.

157. Puigserver, P., et al., A cold-inducible coactivator of nuclear receptors linked to adaptive thermogenesis. Cell, 1998. 92(6): p. 829-39.

158. Wu, Z., et al., Mechanisms controlling mitochondrial biogenesis and respiration through the thermogenic coactivator PGC-1. Cell, 1999. 98(1): p. 115-24.

159. Piantadosi, C.A. and H.B. Suliman, Mitochondrial transcription factor A induction by redox activation of nuclear respiratory factor 1. J Biol Chem, 2006. 281(1): p. 324-33.

160. Virbasius, J.V. and R.C. Scarpulla, Activation of the human mitochondrial transcription factor A gene by nuclear respiratory factors: a potential regulatory link between nuclear and mitochondrial gene expression in organelle biogenesis. Proc Natl Acad Sci U S A, 1994. 91(4): p. 1309-13.

161. Yeh, L.A., K.H. Lee, and K.H. Kim, Regulation of rat liver acetyl-CoA carboxylase. Regulation of phosphorylation and inactivation of acetyl-CoA carboxylase by the adenylate energy charge. J Biol Chem, 1980. 255(6): p. 2308-14.

162. Hardie, D.G., AMP-activated/SNF1 protein kinases: conserved guardians of cellular energy. Nat Rev Mol Cell Biol, 2007. 8(10): p. 774-85.

163. Bergeron, R., et al., Chronic activation of AMP kinase results in NRF-1 activation and mitochondrial biogenesis. Am J Physiol Endocrinol Metab, 2001. 281(6): p. E1340-6.

164. Zong, H., et al., AMP kinase is required for mitochondrial biogenesis in skeletal muscle in response to chronic energy deprivation. Proc Natl Acad Sci U S A, 2002. 99(25): p. 15983-7. 
165. Egawa, T., et al., Involvement of AMPK in regulating slow-twitch muscle atrophy during hindlimb unloading in mice. Am J Physiol Endocrinol Metab, 2015. 309(7): p. E651-62.

166. Gordon, S.E., et al., Does AMP-activated protein kinase negatively mediate aged fast-twitch skeletal muscle mass? Exerc Sport Sci Rev, 2008. 36(4): p. 179-86.

167. Liu, J., et al., Mitochondrial Dysfunction Launches Dexamethasone-Induced Skeletal Muscle Atrophy via AMPK/FOXO3 Signaling. Mol Pharm, 2016. 13(1): p. 73-84.

168. Wang, C. and R.J. Youle, The role of mitochondria in apoptosis*. Annu Rev Genet, 2009. 43: p. 95-118.

169. Cai, J., J. Yang, and D.P. Jones, Mitochondrial control of apoptosis: the role of cytochrome c. Biochim Biophys Acta, 1998. 1366(1-2): p. 139-49.

170. Potts, P.R., et al., Critical function of endogenous XIAP in regulating caspase activation during sympathetic neuronal apoptosis. J Cell Biol, 2003. 163(4): p. 789-99.

171. Willis, S.N., et al., Apoptosis initiated when BH3 ligands engage multiple Bcl-2 homologs, not Bax or Bak. Science, 2007. 315(5813): p. 856-9.

172. Berthelet, J. and L. Dubrez, Regulation of Apoptosis by Inhibitors of Apoptosis (IAPS). Cells, 2013. 2(1): p. 163-87.

173. Jaiswal, N., et al., Fructose induces mitochondrial dysfunction and triggers apoptosis in skeletal muscle cells by provoking oxidative stress. Apoptosis, 2015. 20(7): p. 930-47.

174. Greene, J.C., et al., Mitochondrial pathology and apoptotic muscle degeneration in Drosophila parkin mutants. Proc Natl Acad Sci U S A, 2003. 100(7): p. 407883.

175. Porter, C. and B.T. Wall, Skeletal muscle mitochondrial function: is it quality or quantity that makes the difference in insulin resistance? J Physiol, 2012. 590(23): p. 5935-6.

176. Mancuso, M., et al., Fatigue and exercise intolerance in mitochondrial diseases. Literature revision and experience of the Italian Network of mitochondrial diseases. Neuromuscul Disord, 2012. 22 Suppl 3: p. S226-9.

177. Tomanek, R.J. and D.D. Lund, Degeneration of different types of skeletal muscle fibres. II. Immobilization. J Anat, 1974. 118(Pt 3): p. 531-41.

178. Pressmar, J., et al., Intracellular Ca2+ concentrations are not elevated in resting cultured muscle from Duchenne (DMD) patients and in MDX mouse muscle fibres. Pflugers Arch, 1994. 426(6): p. 499-505.

179. Rosca, M.G. and C.L. Hoppel, Mitochondrial dysfunction in heart failure. Heart Fail Rev, 2013. 18(5): p. 607-22. 
180. Ritov, V.B., et al., Deficiency of subsarcolemmal mitochondria in obesity and type 2 diabetes. Diabetes, 2005. 54(1): p. 8-14.

181. Rosca, M.G., et al., Altered expression of the adenine nucleotide translocase isoforms and decreased ATP synthase activity in skeletal muscle mitochondria in heart failure. J Mol Cell Cardiol, 2009. 46(6): p. 927-35.

182. Takahashi, M. and D.A. Hood, Protein import into subsarcolemmal and intermyofibrillar skeletal muscle mitochondria. Differential import regulation in distinct subcellular regions. J Biol Chem, 1996. 271(44): p. 27285-91.

183. Karbowski, M. and R.J. Youle, Regulating mitochondrial outer membrane proteins by ubiquitination and proteasomal degradation. Curr Opin Cell Biol, 2011. 23(4): p. 476-82.

184. Langer, T., et al., AAA proteases of mitochondria: quality control of membrane proteins and regulatory functions during mitochondrial biogenesis. Biochem Soc Trans, 2001. 29(Pt 4): p. 431-6.

185. Bota, D.A. and K.J. Davies, Lon protease preferentially degrades oxidized mitochondrial aconitase by an ATP-stimulated mechanism. Nat Cell Biol, 2002. 4(9): p. 674-80.

186. Van Dyck, L., D.A. Pearce, and F. Sherman, PIMI encodes a mitochondrial ATP-dependent protease that is required for mitochondrial function in the yeast Saccharomyces cerevisiae. J Biol Chem, 1994. 269(1): p. 238-42.

187. Cipolat, S., et al., Mitochondrial rhomboid PARL regulates cytochrome $c$ release during apoptosis via OPA1-dependent cristae remodeling. Cell, 2006. 126(1): p. 163-75.

188. Jones, J.M., et al., Loss of Omi mitochondrial protease activity causes the neuromuscular disorder of mnd2 mutant mice. Nature, 2003. 425(6959): p. 7217.

189. Moisoi, N., et al., Mitochondrial dysfunction triggered by loss of HtrA2 results in the activation of a brain-specific transcriptional stress response. Cell Death Differ, 2009. 16(3): p. 449-64.

190. Soubannier, V., et al., A vesicular transport pathway shuttles cargo from mitochondria to lysosomes. Curr Biol, 2012. 22(2): p. 135-41.

191. McLelland, G.L., et al., Parkin and PINK1 function in a vesicular trafficking pathway regulating mitochondrial quality control. EMBO J, 2014. 33(4): p. 28295.

192. Soubannier, V., et al., Reconstitution of mitochondria derived vesicle formation demonstrates selective enrichment of oxidized cargo. PLoS One, 2012. 7(12): p. e52830. 
193. Lemasters, J.J., Selective mitochondrial autophagy, or mitophagy, as a targeted defense against oxidative stress, mitochondrial dysfunction, and aging. Rejuvenation Res, 2005. 8(1): p. 3-5.

194. Ni, H.M., J.A. Williams, and W.X. Ding, Mitochondrial dynamics and mitochondrial quality control. Redox Biol, 2015. 4: p. 6-13.

195. Shirihai, O.S., M. Song, and G.W. Dorn, 2nd, How mitochondrial dynamism orchestrates mitophagy. Circ Res, 2015. 116(11): p. 1835-49.

196. Mortensen, M., D.J. Ferguson, and A.K. Simon, Mitochondrial clearance by autophagy in developing erythrocytes: clearly important, but just how much so? Cell Cycle, 2010. 9(10): p. 1901-6.

197. Zhang, Z.W., et al., Red blood cell extrudes nucleus and mitochondria against oxidative stress. IUBMB Life, 2011. 63(7): p. 560-5.

198. Al Rawi, S., et al., Postfertilization autophagy of sperm organelles prevents paternal mitochondrial DNA transmission. Science, 2011. 334(6059): p. 11447.

199. Narendra, D., et al., Parkin is recruited selectively to impaired mitochondria and promotes their autophagy. J Cell Biol, 2008. 183(5): p. 795-803.

200. Kim, I. and J.J. Lemasters, Mitophagy selectively degrades individual damaged mitochondria after photoirradiation. Antioxid Redox Signal, 2011. 14(10): p. 1919-28.

201. Suen, D.F., et al., Parkin overexpression selects against a deleterious mtDNA mutation in heteroplasmic cybrid cells. Proc Natl Acad Sci U S A, 2010. 107(26): p. 11835-40.

202. Song, Z., et al., Mitofusins and OPA1 mediate sequential steps in mitochondrial membrane fusion. Mol Biol Cell, 2009. 20(15): p. 3525-32.

203. Head, B., et al., Inducible proteolytic inactivation of OPA1 mediated by the OMA1 protease in mammalian cells. J Cell Biol, 2009. 187(7): p. 959-66.

204. Poole, A.C., et al., The mitochondrial fusion-promoting factor mitofusin is a substrate of the PINK1/parkin pathway. PLoS One, 2010. 5(4): p. e10054.

205. Tanaka, A., et al., Proteasome and p97 mediate mitophagy and degradation of mitofusins induced by Parkin. J Cell Biol, 2010. 191(7): p. 1367-80.

206. Ziviani, E., R.N. Tao, and A.J. Whitworth, Drosophila parkin requires PINK1 for mitochondrial translocation and ubiquitinates mitofusin. Proc Natl Acad Sci U S A, 2010. 107(11): p. 5018-23.

207. Liu, S. and B. Lu, Reduction of protein translation and activation of autophagy protect against PINK1 pathogenesis in Drosophila melanogaster. PLoS Genet, 2010. 6(12): p. e1001237. 
208. Park, J., et al., Mitochondrial dysfunction in Drosophila PINK1 mutants is complemented by parkin. Nature, 2006. 441(7097): p. 1157-61.

209. Beilina, A., et al., Mutations in PTEN-induced putative kinase 1 associated with recessive parkinsonism have differential effects on protein stability. Proc Natl Acad Sci U S A, 2005. 102(16): p. 5703-8.

210. Seirafi, M., G. Kozlov, and K. Gehring, Parkin structure and function. FEBS J, 2015. 282(11): p. 2076-88.

211. Shiba-Fukushima, K., et al., PINK1-mediated phosphorylation of the Parkin ubiquitin-like domain primes mitochondrial translocation of Parkin and regulates mitophagy. Sci Rep, 2012. 2: p. 1002.

212. Kane, L.A., et al., PINK1 phosphorylates ubiquitin to activate Parkin E3 ubiquitin ligase activity. J Cell Biol, 2014. 205(2): p. 143-53.

213. Koyano, F., et al., Ubiquitin is phosphorylated by PINK1 to activate parkin. Nature, 2014. 510(7503): p. 162-6.

214. Ordureau, A., et al., Defining roles of PARKIN and ubiquitin phosphorylation by PINK1 in mitochondrial quality control using a ubiquitin replacement strategy. Proc Natl Acad Sci U S A, 2015. 112(21): p. 6637-42.

215. Kazlauskaite, A., et al., Phosphorylation of Parkin at Serine65 is essential for activation: elaboration of a Mirol substrate-based assay of Parkin E3 ligase activity. Open Biol, 2014. 4: p. 130213.

216. Glauser, L., et al., Parkin promotes the ubiquitination and degradation of the mitochondrial fusion factor mitofusin 1. J Neurochem, 2011. 118(4): p. 636-45.

217. Lim, K.L., V.L. Dawson, and T.M. Dawson, Parkin-mediated lysine 63-linked polyubiquitination: a link to protein inclusions formation in Parkinson's and other conformational diseases? Neurobiol Aging, 2006. 27(4): p. 524-9.

218. Welchman, R.L., C. Gordon, and R.J. Mayer, Ubiquitin and ubiquitin-like proteins as multifunctional signals. Nat Rev Mol Cell Biol, 2005. 6(8): p. 599609.

219. Chan, N.C., et al., Broad activation of the ubiquitin-proteasome system by Parkin is critical for mitophagy. Hum Mol Genet, 2011. 20(9): p. 1726-37.

220. Yamanaka, K., Y. Sasagawa, and T. Ogura, Recent advances in $p 97 / V C P / C d c 48$ cellular functions. Biochim Biophys Acta, 2012. 1823(1): p. 130-7.

221. Twig, G., et al., Fission and selective fusion govern mitochondrial segregation and elimination by autophagy. EMBO J, 2008. 27(2): p. 433-46.

222. Geisler, S., et al., PINK1/Parkin-mediated mitophagy is dependent on VDAC1 and p62/SQSTM1. Nat Cell Biol, 2010. 12(2): p. 119-31. 
223. Yang, J.Y. and W.Y. Yang, Bit-by-bit autophagic removal of parkin-labelled mitochondria. Nat Commun, 2013. 4: p. 2428.

224. Castillo-Quan, J.I., Parkin' control: regulation of PGC-1alpha through PARIS in Parkinson's disease. Dis Model Mech, 2011. 4(4): p. 427-9.

225. Shin, J.H., et al., PARIS (ZNF746) repression of PGC-1alpha contributes to neurodegeneration in Parkinson's disease. Cell, 2011. 144(5): p. 689-702.

226. Yun, J., et al., MUL1 acts in parallel to the PINK1/parkin pathway in regulating mitofusin and compensates for loss of PINK1/parkin. Elife, 2014. 3: p. e01958.

227. Lou, P.H., et al., Mitochondrial uncouplers with an extraordinary dynamic range. Biochem J, 2007. 407(1): p. 129-40.

228. Wang, Y., et al., ROS-induced mitochondrial depolarization initiates PARK2/PARKIN-dependent mitochondrial degradation by autophagy. Autophagy, 2012. 8(10): p. 1462-76.

229. Chu, C.T., et al., Cardiolipin externalization to the outer mitochondrial membrane acts as an elimination signal for mitophagy in neuronal cells. Nat Cell Biol, 2013. 15(10): p. 1197-1205.

230. Dagda, R.K., et al., Mitochondrially localized ERK2 regulates mitophagy and autophagic cell stress: implications for Parkinson's disease. Autophagy, 2008. 4(6): p. $770-82$.

231. Zhu, J.H., et al., Regulation of autophagy by extracellular signal-regulated protein kinases during 1-methyl-4-phenylpyridinium-induced cell death. Am J Pathol, 2007. 170(1): p. 75-86.

232. Cai, Q., et al., Spatial parkin translocation and degradation of damaged mitochondria via mitophagy in live cortical neurons. Curr Biol, 2012. 22(6): p. 545-52.

233. Lazarou, M., et al., The ubiquitin kinase PINK1 recruits autophagy receptors to induce mitophagy. Nature, 2015. 524(7565): p. 309-314.

234. Baker, M.G. and L. Graham, The journey: Parkinson's disease. BMJ, 2004. 329(7466): p. 611-4.

235. Winklhofer, K.F. and C. Haass, Mitochondrial dysfunction in Parkinson's disease. Biochim Biophys Acta, 2010. 1802(1): p. 29-44.

236. Davis, G.C., et al., Chronic Parkinsonism secondary to intravenous injection of meperidine analogues. Psychiatry Res, 1979. 1(3): p. 249-54.

237. Langston, J.W., et al., Chronic Parkinsonism in humans due to a product of meperidine-analog synthesis. Science, 1983. 219(4587): p. 979-80.

238. Bove, J., et al., Toxin-induced models of Parkinson's disease. NeuroRx, 2005. 2(3): p. 484-94. 
239. Kitada, T., et al., Mutations in the parkin gene cause autosomal recessive juvenile parkinsonism. Nature, 1998. 392(6676): p. 605-8.

240. Valente, E.M., et al., PINK1 mutations are associated with sporadic early-onset parkinsonism. Ann Neurol, 2004. 56(3): p. 336-41.

241. Lucking, C.B., et al., Association between early-onset Parkinson's disease and mutations in the parkin gene. N Engl J Med, 2000. 342(21): p. 1560-7.

242. Rial, D., et al., Behavioral phenotyping of Parkin-deficient mice: looking for early preclinical features of Parkinson's disease. PLoS One, 2014. 9(12): p. e114216.

243. Perez, F.A. and R.D. Palmiter, Parkin-deficient mice are not a robust model of parkinsonism. Proc Natl Acad Sci U S A, 2005. 102(6): p. 2174-9.

244. Goldberg, M.S., et al., Parkin-deficient mice exhibit nigrostriatal deficits but not loss of dopaminergic neurons. J Biol Chem, 2003. 278(44): p. 43628-35.

245. Oyama, G., et al., Impaired in vivo dopamine release in parkin knockout mice. Brain Res, 2010. 1352: p. 214-22.

246. Rosen, K.M., et al., Parkin protects against mitochondrial toxins and betaamyloid accumulation in skeletal muscle cells. J Biol Chem, 2006. 281(18): p. 12809-16.

247. Furuya, N., et al., PARK2/Parkin-mediated mitochondrial clearance contributes to proteasome activation during slow-twitch muscle atrophy via NFE2L1 nuclear translocation. Autophagy, 2014. 10(4): p. 631-41.

248. Drew, B.G., et al., HSP72 is a mitochondrial stress sensor critical for Parkin action, oxidative metabolism, and insulin sensitivity in skeletal muscle. Diabetes, 2014. 63(5): p. 1488-505.

249. Blin, O., et al., Mitochondrial respiratory failure in skeletal muscle from patients with Parkinson's disease and multiple system atrophy. J Neurol Sci, 1994. 125(1): p. 95-101.

250. Duvoisin, R.C., Overview of Parkinson's disease. Ann N Y Acad Sci, 1992. 648: p. 187-93.

251. Dauer, W. and S. Przedborski, Parkinson's disease: mechanisms and models. Neuron, 2003. 39(6): p. 889-909.

252. Askanas, V. and W.K. Engel, Inclusion-body myositis: muscle-fiber molecular pathology and possible pathogenic significance of its similarity to Alzheimer's and Parkinson's disease brains. Acta Neuropathol, 2008. 116(6): p. 583-95.

253. Askanas, V. and W.K. Engel, Sporadic inclusion-body myositis: conformational multifactorial ageing-related degenerative muscle disease associated with proteasomal and lysosomal inhibition, endoplasmic reticulum stress, and 
accumulation of amyloid-beta42 oligomers and phosphorylated tau. Presse Med, 2011. 40(4 Pt 2): p. e219-35.

254. Barichella, M., et al., Sarcopenia and Dynapenia in Patients With Parkinsonism. J Am Med Dir Assoc, 2016. 17(7): p. 640-6.

255. Penn, A.M., et al., Generalized mitochondrial dysfunction in Parkinson's disease detected by magnetic resonance spectroscopy of muscle. Neurology, 1995. 45(11): p. 2097-9.

256. Winkler-Stuck, K., et al., Re-evaluation of the dysfunction of mitochondrial respiratory chain in skeletal muscle of patients with Parkinson's disease. J Neural Transm (Vienna), 2005. 112(4): p. 499-518.

257. Falvo, M.J., B.K. Schilling, and G.M. Earhart, Parkinson's disease and resistive exercise: rationale, review, and recommendations. Mov Disord, 2008. 23(1): p. $1-11$.

258. Garber, C.E. and J.H. Friedman, Effects of fatigue on physical activity and function in patients with Parkinson's disease. Neurology, 2003. 60(7): p. 111924.

259. Stevens-Lapsley, J., B.M. Kluger, and M. Schenkman, Quadriceps muscle weakness, activation deficits, and fatigue with Parkinson disease. Neurorehabil Neural Repair, 2012. 26(5): p. 533-41.

260. Tamura, Y., et al., Daily heat stress treatment rescues denervation-activated mitochondrial clearance and atrophy in skeletal muscle. J Physiol, 2015. 593(12): p. 2707-20.

261. Chen, C., et al., Contribution of neural cell death to depressive phenotypes of streptozotocin-induced diabetic mice. Dis Model Mech, 2014. 7(6): p. 723-30.

262. Garcia-Ruiz, I., et al., High-fat diet decreases activity of the oxidative phosphorylation complexes and causes nonalcoholic steatohepatitis in mice. Dis Model Mech, 2014. 7(11): p. 1287-96.

263. Bronner, D.N., et al., Endoplasmic Reticulum Stress Activates the Inflammasome via NLRP3- and Caspase-2-Driven Mitochondrial Damage. Immunity, 2015. 43(3): p. 451-62.

264. Yaffe, D. and O. Saxel, Serial passaging and differentiation of myogenic cells isolated from dystrophic mouse muscle. Nature, 1977. 270(5639): p. 725-7.

265. Tannu, N.S., et al., Comparative proteomes of the proliferating $C(2) C(12)$ myoblasts and fully differentiated myotubes reveal the complexity of the skeletal muscle differentiation program. Mol Cell Proteomics, 2004. 3(11): p. 1065-82.

266. Bouman, L., et al., Parkin is transcriptionally regulated by ATF4: evidence for an interconnection between mitochondrial stress and ER stress. Cell Death Differ, 2011. 18(5): p. 769-82. 
267. Burchell, V.S., et al., The Parkinson's disease-linked proteins Fbxo7 and Parkin interact to mediate mitophagy. Nat Neurosci, 2013. 16(9): p. 1257-65.

268. Carroll, R.G., E. Hollville, and S.J. Martin, Parkin sensitizes toward apoptosis induced by mitochondrial depolarization through promoting degradation of Mcl-1. Cell Rep, 2014. 9(4): p. 1538-53.

269. Gomez-Sanchez, R., et al., Mitochondrial impairment increases FL-PINK1 levels by calcium-dependent gene expression. Neurobiol Dis, 2014. 62: p. 42640.

270. Hardie, D.G., F.A. Ross, and S.A. Hawley, AMPK: a nutrient and energy sensor that maintains energy homeostasis. Nat Rev Mol Cell Biol, 2012. 13(4): p. 25162.

271. Shirwany, N.A. and M.H. Zou, AMPK: a cellular metabolic and redox sensor. A minireview. Front Biosci (Landmark Ed), 2014. 19: p. 447-74.

272. Leal, S.S. and C.M. Gomes, Calcium dysregulation links ALS defective proteins and motor neuron selective vulnerability. Front Cell Neurosci, 2015. 9: p. 225.

273. Sandri, M., et al., Foxo transcription factors induce the atrophy-related ubiquitin ligase atrogin-1 and cause skeletal muscle atrophy. Cell, 2004. 117(3): p. 399-412.

274. Stitt, T.N., et al., The IGF-1/PI3K/Akt pathway prevents expression of muscle atrophy-induced ubiquitin ligases by inhibiting FOXO transcription factors. Mol Cell, 2004. 14(3): p. 395-403.

275. Ogasawara, R., et al., The role of mTOR signalling in the regulation of skeletal muscle mass in a rodent model of resistance exercise. Sci Rep, 2016. 6: p. 31142.

276. You, J.S., et al., The role of $m$ TOR signaling in the regulation of protein synthesis and muscle mass during immobilization in mice. Dis Model Mech, 2015. 8(9): p. 1059-69.

277. Antony, P.M., et al., The hallmarks of Parkinson's disease. FEBS J, 2013. 280(23): p. 5981-93.

278. Piao, Y., et al., Overexpression of TFAM, NRF-1 and myr-AKT protects the $M P P(+)$-induced mitochondrial dysfunctions in neuronal cells. Biochim Biophys Acta, 2012. 1820(5): p. 577-85.

279. Bruggemann, N. and C. Klein, Parkin Type of Early-Onset Parkinson Disease, in GeneReviews(R), R.A. Pagon, et al., Editors. 1993: Seattle (WA).

280. Grunewald, A., et al., Mutant Parkin impairs mitochondrial function and morphology in human fibroblasts. PLoS One, 2010. 5(9): p. e12962.

281. Lee, J.Y., et al., Disease-causing mutations in parkin impair mitochondrial ubiquitination, aggregation, and HDAC6-dependent mitophagy. J Cell Biol, 2010. 189(4): p. 671-9. 
282. Pacelli, C., et al., Mitochondrial defect and PGC-1alpha dysfunction in parkinassociated familial Parkinson's disease. Biochim Biophys Acta, 2011. 1812(8): p. 1041-53.

283. Minamikawa, T., et al., Mitochondrial permeability transition and swelling can occur reversibly without inducing cell death in intact human cells. Exp Cell Res, 1999. 246(1): p. 26-37.

284. Lim, M.L., T. Minamikawa, and P. Nagley, The protonophore CCCP induces mitochondrial permeability transition without cytochrome $c$ release in human osteosarcoma cells. FEBS Lett, 2001. 503(1): p. 69-74.

285. Sargsyan, A., et al., Rapid parallel measurements of macroautophagy and mitophagy in mammalian cells using a single fluorescent biosensor. Sci Rep, 2015. 5: p. 12397.

286. Greene, A.W., et al., Mitochondrial processing peptidase regulates PINK1 processing, import and Parkin recruitment. EMBO Rep, 2012. 13(4): p. 378-85.

287. Senf, S.M., S.L. Dodd, and A.R. Judge, FOXO signaling is required for disuse muscle atrophy and is directly regulated by Hsp70. Am J Physiol Cell Physiol, 2010. 298(1): p. C38-45.

288. Hurley, J.H. and E. Nogales, Next-generation electron microscopy in autophagy research. Curr Opin Struct Biol, 2016. 41: p. 211-216.

289. Yla-Anttila, P., et al., Monitoring autophagy by electron microscopy in Mammalian cells. Methods Enzymol, 2009. 452: p. 143-64.

290. Gegg, M.E. and A.H. Schapira, PINK1-parkin-dependent mitophagy involves ubiquitination of mitofusins 1 and 2: Implications for Parkinson disease pathogenesis. Autophagy, 2011. 7(2): p. 243-5.

291. Ulusoy, A. and D. Kirik, Can overexpression of parkin provide a novel strategy for neuroprotection in Parkinson's disease? Exp Neurol, 2008. 212(2): p. 25860.

292. Rana, A., M. Rera, and D.W. Walker, Parkin overexpression during aging reduces proteotoxicity, alters mitochondrial dynamics, and extends lifespan. Proc Natl Acad Sci U S A, 2013. 110(21): p. 8638-43.

293. Ishihara, N., et al., Mitochondrial fission factor Drpl is essential for embryonic development and synapse formation in mice. Nat Cell Biol, 2009. 11(8): p. 95866.

294. Sin, J., et al., Mitophagy is required for mitochondrial biogenesis and myogenic differentiation of C2C12 myoblasts. Autophagy, 2016. 12(2): p. 369-80.

295. Kageyama, Y., et al., Parkin-independent mitophagy requires Drpl and maintains the integrity of mammalian heart and brain. EMBO J, 2014. 33(23): p. 2798-813. 
296. Lutz, A.K., et al., Loss of parkin or PINK1 function increases Drp1-dependent mitochondrial fragmentation. J Biol Chem, 2009. 284(34): p. 22938-51.

297. Chen, H., et al., Mitofusins Mfn1 and Mfn2 coordinately regulate mitochondrial fusion and are essential for embryonic development. J Cell Biol, 2003. 160(2): p. 189-200.

298. Chen, Y., et al., Mitofusin 2-containing mitochondrial-reticular microdomains direct rapid cardiomyocyte bioenergetic responses via interorganelle $\mathrm{Ca}(2+)$ crosstalk. Circ Res, 2012. 111(7): p. 863-75.

299. Rodriguez-Navarro, J.A., et al., Mortality, oxidative stress and tau accumulation during ageing in parkin null mice. J Neurochem, 2007. 103(1): p. 98-114.

300. Nicklas, W.J., I. Vyas, and R.E. Heikkila, Inhibition of NADH-linked oxidation in brain mitochondria by 1-methyl-4-phenyl-pyridine, a metabolite of the neurotoxin, 1-methyl-4-phenyl-1,2,5,6-tetrahydropyridine. Life Sci, 1985. 36(26): p. 2503-8.

301. Ramsay, R.R., et al., Inhibition of mitochondrial NADH dehydrogenase by pyridine derivatives and its possible relation to experimental and idiopathic parkinsonism. Biochem Biophys Res Commun, 1986. 135(1): p. 269-75.

302. Reinecke, F., J.A. Smeitink, and F.H. van der Westhuizen, OXPHOS gene expression and control in mitochondrial disorders. Biochim Biophys Acta, 2009. 1792(12): p. 1113-21.

303. Handy, D.E. and J. Loscalzo, Redox regulation of mitochondrial function. Antioxid Redox Signal, 2012. 16(11): p. 1323-67.

304. Miwa, S., et al., Low abundance of the matrix arm of complex I in mitochondria predicts longevity in mice. Nat Commun, 2014. 5: p. 3837.

305. Dodd, S.L., et al., Ros-mediated activation of NF-kappaB and Foxo during muscle disuse. Muscle Nerve, 2010. 41(1): p. 110-3.

306. McClung, J.M., et al., p38 MAPK links oxidative stress to autophagy-related gene expression in cachectic muscle wasting. Am J Physiol Cell Physiol, 2010. 298(3): p. C542-9.

307. McClung, J.M., et al., Calpain-1 is required for hydrogen peroxide-induced myotube atrophy. Am J Physiol Cell Physiol, 2009. 296(2): p. C363-71.

308. Kalyanaraman, B., et al., Measuring reactive oxygen and nitrogen species with fluorescent probes: challenges and limitations. Free Radic Biol Med, 2012. 52(1): p. 1-6.

309. Polster, B.M., et al., Use of potentiometric fluorophores in the measurement of mitochondrial reactive oxygen species. Methods Enzymol, 2014. 547: p. 22550. 
310. Steinberg, G.R. and B.E. Kemp, AMPK in Health and Disease. Physiol Rev, 2009. 89(3): p. 1025-78.

311. Kim, T.W., et al., (ADP-ribose) polymerase 1 and AMP-activated protein kinase mediate progressive dopaminergic neuronal degeneration in a mouse model of Parkinson's disease. Cell Death Dis, 2013. 4: p. e919.

312. $\mathrm{Xu}, \mathrm{Y} .$, et al., Activation of AMPK and inactivation of Akt result in suppression of mTOR-mediated S6K1 and 4E-BP1 pathways leading to neuronal cell death in in vitro models of Parkinson's disease. Cell Signal, 2014. 26(8): p. 1680-9.

313. Wu, Y., et al., Resveratrol-activated AMPK/SIRT1/autophagy in cellular models of Parkinson's disease. Neurosignals, 2011. 19(3): p. 163-74.

314. Romanello, V. and M. Sandri, Mitochondrial Quality Control and Muscle Mass Maintenance. Front Physiol, 2015. 6: p. 422.

315. Jheng, H.F., et al., Mitochondrial fission contributes to mitochondrial dysfunction and insulin resistance in skeletal muscle. Mol Cell Biol, 2012.32(2): p. 309-19.

316. Powers, S.K., et al., Mitochondrial signaling contributes to disuse muscle atrophy. Am J Physiol Endocrinol Metab, 2012. 303(1): p. E31-9.

317. Guha, M., et al., Activation of Akt is essential for the propagation of mitochondrial respiratory stress signaling and activation of the transcriptional coactivator heterogeneous ribonucleoprotein A2. Mol Biol Cell, 2010. 21(20): p. 3578-89.

318. Liang, R., et al., Evidence for AKT-independent regulation of FOXO1 and FOXO3 in haematopoietic stem and progenitor cells. Cell Cycle, 2016. 15(6): p. 861-7.

319. Tzivion, G., M. Dobson, and G. Ramakrishnan, FoxO transcription factors; Regulation by AKT and 14-3-3 proteins. Biochim Biophys Acta, 2011. 1813(11): p. 1938-45.

320. Lang, A.E. and A.M. Lozano, Parkinson's disease. First of two parts. N Engl J Med, 1998. 339(15): p. 1044-53.

321. Fontana, S., et al., Reduction in mdx mouse muscle degeneration by low-intensity endurance exercise: a proteomic analysis in quadriceps muscle of exercised compared with sedentary mdx mice. Biosci Rep, 2015. 35(3).

322. Nishita, T., et al., Muscle carbonic anhydrase III levels in normal and muscular dystrophia afflicted chickens. Acta Vet Scand, 2012. 54: p. 34.

323. Zheng, Y., L. Dai, and W. Fu, [Carbonic anhydrase III and mRNA expression levels in quadriceps femoris muscle of chronic obstructive pulmonary disease patients]. Zhonghua Nei Ke Za Zhi, 2014. 53(7): p. 555-7. 
324. Heath, R., et al., Fetal plasma carbonic anhydrase III in prenatal diagnosis of Duchenne muscular dystrophy. Am J Med Genet, 1985. 20(1): p. 115-22.

325. Zimmerman, U.J., et al., Anti-oxidative response of carbonic anhydrase III in skeletal muscle. IUBMB Life, 2004. 56(6): p. 343-7.

326. Krawiec, B.J., et al., AMP-activated protein kinase agonists increase mRNA content of the muscle-specific ubiquitin ligases MAFbx and MuRF1 in C2C12 cells. Am J Physiol Endocrinol Metab, 2007. 292(6): p. E1555-67.

327. Nakashima, K. and Y. Yakabe, AMPK activation stimulates myofibrillar protein degradation and expression of atrophy-related ubiquitin ligases by increasing FOXO transcription factors in C2C12 myotubes. Biosci Biotechnol Biochem, 2007. 71(7): p. 1650-6.

328. Abumrad, N.A. and D.J. Moore, Parkin reinvents itself to regulate fatty acid metabolism by tagging CD36. J Clin Invest, 2011. 121(9): p. 3389-92.

329. Kim, K.Y., et al., Parkin is a lipid-responsive regulator of fat uptake in mice and mutant human cells. J Clin Invest, 2011. 121(9): p. 3701-12.

330. Polge, C., D. Attaix, and D. Taillandier, Role of E2-Ub-conjugating enzymes during skeletal muscle atrophy. Front Physiol, 2015. 6: p. 59.

331. Townsley, F.M., et al., Dominant-negative cyclin-selective ubiquitin carrier protein E2-C/UbcH10 blocks cells in metaphase. Proc Natl Acad Sci U S A, 1997. 94(6): p. 2362-7.

332. Okamoto, Y., et al., UbcH10 is the cancer-related E2 ubiquitin-conjugating enzyme. Cancer Res, 2003. 63(14): p. 4167-73.

333. Meza-Gutierrez, F., F.V. Hundley, and D.P. Toczyski, Parallel Parkin: $C d c 20$ Takes a New Partner. Mol Cell, 2015. 60(1): p. 3-4.

334. Lee, S.B., et al., Parkin Regulates Mitosis and Genomic Stability through Cdc20/Cdhl. Mol Cell, 2015. 60(1): p. 21-34.

335. Spaich, S., et al., F-box and leucine-rich repeat protein 22 is a cardiac-enriched F-box protein that regulates sarcomeric protein turnover and is essential for maintenance of contractile function in vivo. Circ Res, 2012. 111(12): p. 150416.

336. Ho, S.R., et al., RNF144A, an E3 ubiquitin ligase for DNA-PKcs, promotes apoptosis during DNA damage. Proc Natl Acad Sci U S A, 2014. 111(26): p. E2646-55.

337. Ng, C.C., et al., p53RFP, a p53-inducible RING-finger protein, regulates the stability of p21WAF1. Oncogene, 2003. 22(28): p. 4449-58.

338. Zhang, X., et al., Molecular responses of macrophages to porcine reproductive and respiratory syndrome virus infection. Virology, 1999. 262(1): p. 152-62. 
339. Li, X.L., et al., RNase-L-dependent destabilization of interferon-induced mRNAs. A role for the 2-5A system in attenuation of the interferon response. $\mathrm{J}$ Biol Chem, 2000. 275(12): p. 8880-8.

340. Malakhova, O., et al., Lipopolysaccharide activates the expression of ISG15specific protease UBP43 via interferon regulatory factor 3. J Biol Chem, 2002. 277(17): p. 14703-11.

341. Peng, Q.L., et al., Transcriptomic profiling of long non-coding RNAs in dermatomyositis by microarray analysis. Sci Rep, 2016. 6: p. 32818.

342. Parent, M. and A. Parent, Substantia nigra and Parkinson's disease: a brief history of their long and intimate relationship. Can J Neurol Sci, 2010. 37(3): p. 313-9.

343. Parkinson, J., An essay on the shaking palsy. 1817. J Neuropsychiatry Clin Neurosci, 2002. 14(2): p. 223-36; discussion 222.

344. Tanaka, A., Parkin-mediated selective mitochondrial autophagy, mitophagy: Parkin purges damaged organelles from the vital mitochondrial network. FEBS Lett, 2010. 584(7): p. 1386-92.

345. Halbert, C.L., J.M. Allen, and A.D. Miller, Adeno-associated virus type 6 (AAV6) vectors mediate efficient transduction of airway epithelial cells in mouse lungs compared to that of AAV2 vectors. J Virol, 2001. 75(14): p. 6615-24.

346. Deng, H., et al., The Parkinson's disease genes pinkl and parkin promote mitochondrial fission and/or inhibit fusion in Drosophila. Proc Natl Acad Sci U S A, 2008. 105(38): p. 14503-8.

347. Poole, A.C., et al., The PINK1/Parkin pathway regulates mitochondrial morphology. Proc Natl Acad Sci U S A, 2008. 105(5): p. 1638-43.

348. Yang, Y., et al., Pink1 regulates mitochondrial dynamics through interaction with the fission/fusion machinery. Proc Natl Acad Sci U S A, 2008. 105(19): p. 7070-5.

349. Mogi, M., et al., Interleukin-1 beta, interleukin-6, epidermal growth factor and transforming growth factor-alpha are elevated in the brain from parkinsonian patients. Neurosci Lett, 1994. 180(2): p. 147-50.

350. Fry, C.S., et al., Glucocorticoids increase skeletal muscle NF-kappaB inducing kinase (NIK): links to muscle atrophy. Physiol Rep, 2016. 4(21).

351. Becker, D.E., Basic and clinical pharmacology of glucocorticosteroids. Anesth Prog, 2013. 60(1): p. 25-31; quiz 32.

352. Riley, B.E., et al., Structure and function of Parkin E3 ubiquitin ligase reveals aspects of RING and HECT ligases. Nat Commun, 2013. 4: p. 1982. 
353. Heo, J.M., et al., The PINK1-PARKIN Mitochondrial Ubiquitylation Pathway Drives a Program of OPTN/NDP52 Recruitment and TBK1 Activation to Promote Mitophagy. Mol Cell, 2015. 60(1): p. 7-20.

354. Kitaoka, Y., et al., Nrf2 deficiency does not affect denervation-induced alterations in mitochondrial fission and fusion proteins in skeletal muscle. Physiol Rep, 2016. 4(24).

355. Hoshino, A., et al., Cytosolic p53 inhibits Parkin-mediated mitophagy and promotes mitochondrial dysfunction in the mouse heart. Nat Commun, 2013. 4: p. 2308. 